\title{
5. Pedological, floristic and mycological characteristics of the study areas
}

\subsection{Abandoned land study plot groups in the buffer zone around landscape parks on the Pilica River}

\author{
Stanisław Krysiak, Jolanta Adamczyk, Jarosław Sieradzki
}

\section{GLINNIK STUDY PLOT GROUP}

The Glinnik study plot group is located several hundred metres from the borders of the Spała Landscape Park, in Lubochnia Commune. It is surrounded by extensive forest complexes from the east and south. Surface formations include upper fluvioglacial sands and gravels that originated during the cataglacial phase of the Warta Glaciation (Trzmiel 1986). A small addition of silty fraction, found in the uppermost series of the Glinnik A, Glinnik B and Glinnik C study plots is related to aeolian accumulation in the periglacial conditions of the Vistulian. The formations constitute the parent rock for sandy soils of different genetic types $(\mathrm{AB})$, whose agricultural suitability was classified as poor rye complex (6) and very poor rye complex (7) (Województwo piotrkowskie. Mapa glebowo-rolnicza 1979). As regards soil valuation, the productivity of soils at plots Glinnik A and Glinnik C was classified as class VI, whereas plot Glinnik B - as class V of arable lands (http:// geoportal.lodzkie.pl/imap/). Photographic documentation and results of laboratory analyses from the Glinnik study plot group are presented in figures 5.1-5.6, and in tables 5.1-5.3.

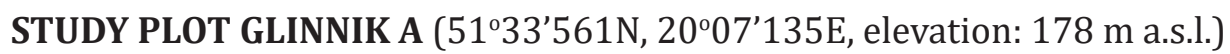
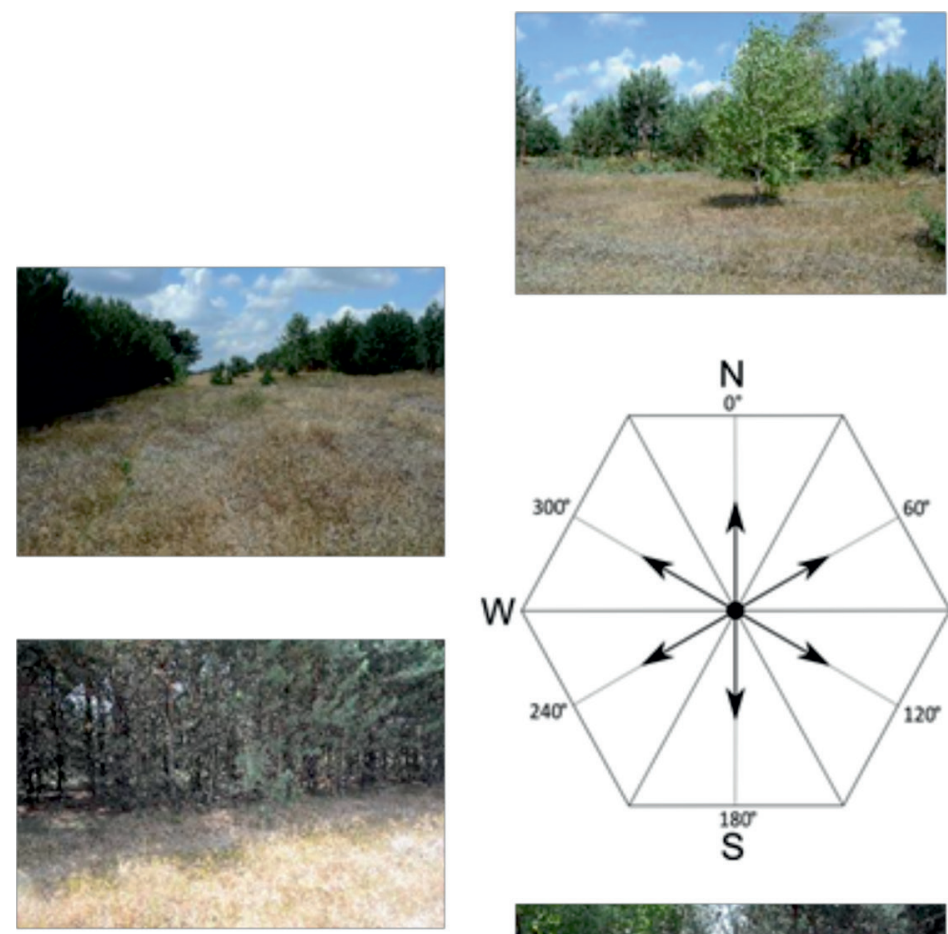
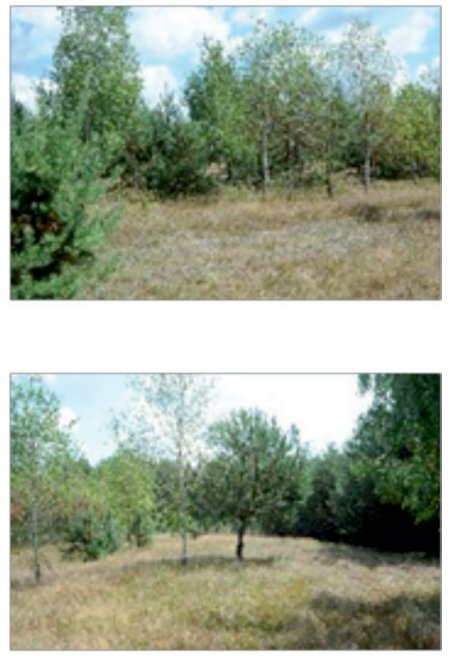

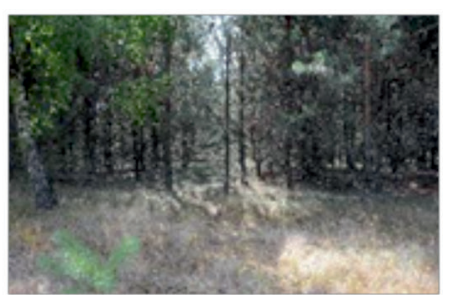

Fig. 5.1. Study plot Glinnik A (photo S. Krysiak, 2012) 


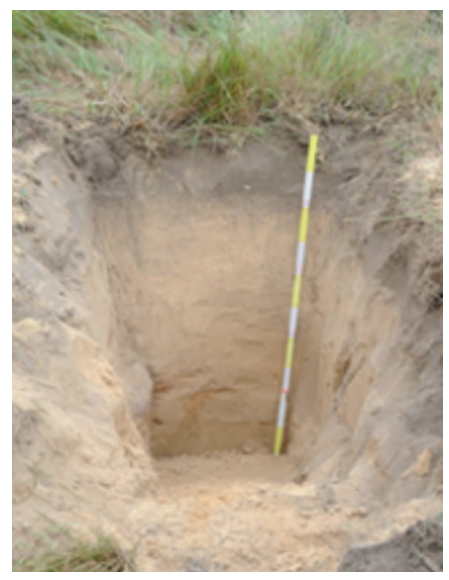

\author{
Depth Profile description \\ $0-23 \mathrm{~cm}$ - humus, fine and silty sand, grey \\ 23-70 cm - fine and silty sand, light beige \\ $70-90 \mathrm{~cm}$ - fine sand with a boulder (20 cm in diameter) and cobbles \\ 90-110 cm - medium and fine sand, rusty yellow
}

Fig. 5.2. Soil pit in study plot Glinnik A (photo S. Krysiak, 2012)

Table 5.1. Study plot Glinnik A. Granulometric and chemical properties of soil

\begin{tabular}{|c|c|c|c|c|c|c|c|c|c|c|c|c|}
\hline \multirow[b]{2}{*}{ Soil pit } & \multirow{2}{*}{$\begin{array}{c}\text { Sampling } \\
\text { depth } \\
\text { cm }\end{array}$} & \multicolumn{9}{|c|}{$\begin{array}{c}\text { Grain size distribution } \\
\%\end{array}$} & \multirow{2}{*}{$\begin{array}{l}\text { Granu- } \\
\text { lome- } \\
\text { tric } \\
\text { sub- } \\
\text { group }\end{array}$} & \multirow{2}{*}{$\begin{array}{c}\text { Specific } \\
\text { surface } \\
\text { area } \\
\mathrm{m}^{2} \cdot \mathrm{g}^{-1}\end{array}$} \\
\hline & & $\begin{array}{c}2.0- \\
1.0 \\
\mathrm{~mm}\end{array}$ & $\begin{array}{c}1.0- \\
0.5 \\
\mathrm{~mm}\end{array}$ & $\begin{array}{l}0.5- \\
0.25 \\
\mathrm{~mm}\end{array}$ & $\begin{array}{c}0.25- \\
0.1 \\
\mathrm{~mm}\end{array}$ & $\begin{array}{l}0.1- \\
0.05 \\
\mathrm{~mm}\end{array}$ & $\begin{array}{c}0.05- \\
0.02 \\
\mathrm{~mm}\end{array}$ & $\begin{array}{c}0.02- \\
0.005 \\
\mathrm{~mm}\end{array}$ & $\begin{array}{c}0.005- \\
0.002 \\
\mathrm{~mm}\end{array}$ & $\begin{array}{c}<0.002 \\
\mathrm{~mm}\end{array}$ & & \\
\hline Glinnik A & $0-20$ & 0.0 & 20.8 & 43.0 & 15.3 & 5.7 & 8.5 & 4.6 & 1.3 & 0.8 & ps & 0.1350 \\
\hline Glinnik A & $20-40$ & 0.0 & 19.7 & 42.2 & 17.8 & 5.6 & 7.6 & 4.5 & 1.4 & 1.1 & ps & 0.1550 \\
\hline Glinnik A & $90-110$ & 0.0 & 18.3 & 72.9 & 7.2 & 0.0 & 0.0 & 1.0 & 0.5 & 0.1 & $\mathrm{pl}$ & 0.0344 \\
\hline
\end{tabular}

\begin{tabular}{|c|c|c|c|c|c|c|c|c|c|c|}
\hline \multirow[t]{2}{*}{ Soil pit } & \multirow{2}{*}{$\begin{array}{c}\text { Sampling } \\
\text { depth } \\
\mathrm{cm}\end{array}$} & \multirow{2}{*}{$\begin{array}{c}\text { Humus } \\
\%\end{array}$} & \multirow{2}{*}{$\begin{array}{l}\text { C total } \\
\%\end{array}$} & \multirow{2}{*}{$\begin{array}{l}\mathrm{N} \text { total } \\
\%\end{array}$} & \multirow[t]{2}{*}{$\mathrm{C} / \mathrm{N}$} & \multicolumn{2}{|c|}{$\begin{array}{l}\mathrm{pH} \\
\text { in }\end{array}$} & \multicolumn{3}{|c|}{$\begin{array}{l}\text { Available nutrients } \\
\mathrm{mg} / 100 \mathrm{~g}\end{array}$} \\
\hline & & & & & & $\mathrm{KCl}$ & $\mathrm{H}_{2} \mathrm{O}$ & $\mathrm{P}_{2} \mathrm{O}_{5}$ & $\mathrm{~K}_{2} \mathrm{O}$ & $\mathrm{Mg}$ \\
\hline Glinnik A & $0-20$ & 1.75 & 1.015 & 0.069 & 14.71 & 4.0 & 4.8 & 5.0 & 2.6 & 0.4 \\
\hline Glinnik A & $20-40$ & - & - & - & - & 4.3 & 4.9 & 3.0 & 1.5 & 0.2 \\
\hline Glinnik A & $90-110$ & - & - & - & - & 4.3 & 5.2 & 1.6 & 1.6 & 0.4 \\
\hline
\end{tabular}

\begin{tabular}{|c|c|c|c|c|c|c|c|c|c|c|}
\hline \multirow{2}{*}{ Soil pit } & \multirow{2}{*}{$\begin{array}{c}\text { Sampling } \\
\text { depth } \\
\text { cm }\end{array}$} & \multirow{2}{*}{$\begin{array}{l}\text { Hydrolytic } \\
\text { acidity } \\
\text { mmol/100g }\end{array}$} & \multicolumn{4}{|c|}{$\begin{array}{c}\text { Exchangeable cations } \\
\text { me } / 100 \mathrm{~g}\end{array}$} & \multicolumn{4}{|c|}{$\begin{array}{c}\text { Sorption capacity } \\
\text { me } / 100 \mathrm{~g}\end{array}$} \\
\hline & & & $\mathrm{Ca}^{2+}$ & $\mathrm{Mg}^{2+}$ & $\mathrm{Na}^{+}$ & $\mathrm{K}^{+}$ & S & $\mathrm{T}$ & $(\mathrm{T}-\mathrm{S})$ & $\mathrm{V}, \%$ \\
\hline Glinnik A & $0-20$ & 4.75 & 0.15 & 0.020 & 0.017 & 0.079 & 0.266 & 5.016 & 4.75 & 5.5 \\
\hline Glinnik A & $20-40$ & 2.27 & 0.10 & 0.013 & 0.009 & 0.044 & 0.166 & 2.436 & 2.27 & 6.8 \\
\hline Glinnik A & $90-110$ & 1.45 & 0.10 & 0.027 & 0.009 & 0.031 & 0.167 & 1.617 & 1.45 & 10.3 \\
\hline
\end{tabular}

Source: own elaboration.

\title{
Characteristics of the flora and fungi - Glinnik A
}

Approximately $100 \%$ of the study plot is covered with plants. Most recorded species are grasses. The share of dicotyledons is smaller. Two species dominate among plants: Agrostis capillaris (about $65 \%$ ) and Hieracium pilosella (about 25\%). The share of other species does not exceed $0.5 \%$ of land cover. Identified grass species include: $P o a$ pratensis, Anthoxantum odoratum, Festuca ovina, Apera spica-venti, Nardus stricta, Arrenatherum elatius. Other observed species include e.g.: Jasio- 
ne montana, Achillea millefolium (Tab. 6.1). Sparse saplings of trees are also found in the area: Betula pendula, Pinus sylvestris and Quercus robur.

Fungi of the plot are represented by 17 species of macromycetes. The most frequently found are
Bovista plumbea and Crinipellis scabella, which appear on grasses, mostly on Agrostis capillaris and Festuca ovina. Among land mycorrhizal fungi, the most abundant species was Amanita muscaria (Tab. 7.1).

STUDY PLOT GLINNIK B (51 $33^{\prime} 449 \mathrm{~N}, 20^{\circ} 07^{\prime} 114 \mathrm{E}$, elevation $172 \mathrm{~m}$ a.s.l.)
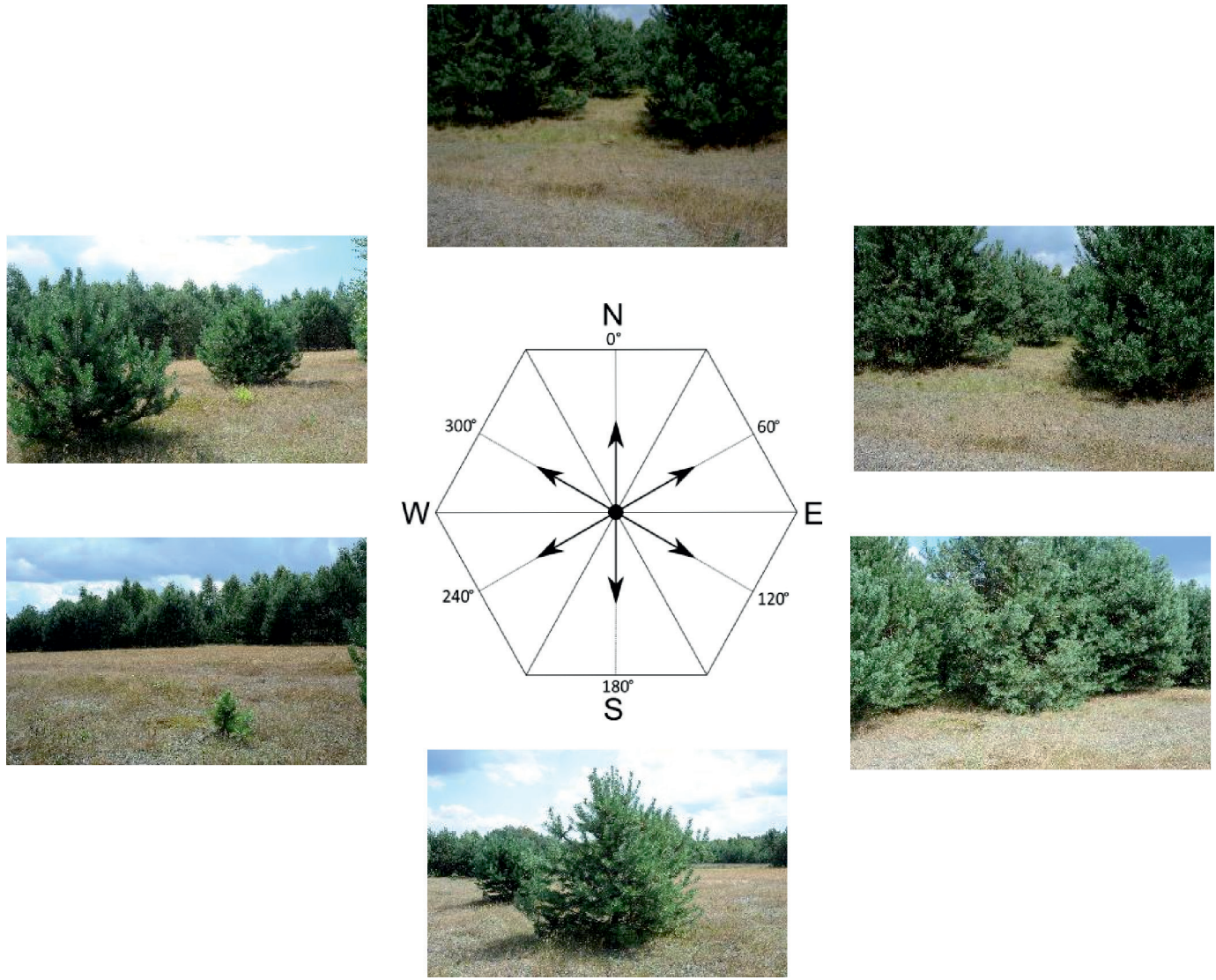

Fig. 5.3. Study plot Glinnik B (photo S. Krysiak, 2012)

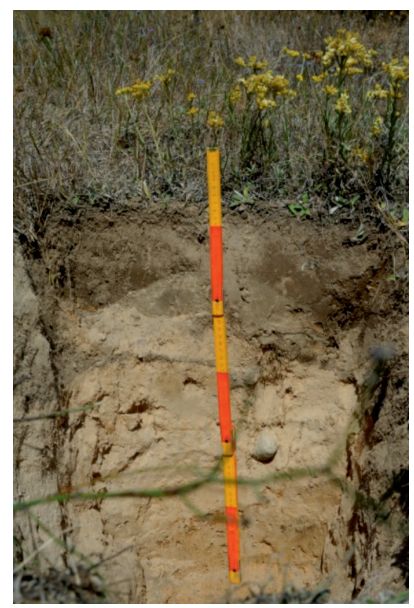

$$
\begin{aligned}
\text { Depth } & \text { Profile description } \\
0-20 \mathrm{~cm} & - \text { humus, fine and silty sand, grey } \\
20-40 \mathrm{~cm} & - \text { unsorted sand with single pebbles, yellow } \\
40-70 \mathrm{~cm} & - \text { unsorted sand with gravel and cobbles of } 3-4 \mathrm{~cm} \\
& \text { in diameter, rusty } \\
70-100 \mathrm{~cm} & - \text { fine sand with cobbles, yellow } \\
100-110 \mathrm{~cm} & - \text { loamy sand, brown }
\end{aligned}
$$

Fig. 5.4. Soil pit in study plot Glinnik B (photo S. Krysiak, 2012) 
Table 5.2. Study plot Glinnik B. Granulometric and chemical properties of soil

\begin{tabular}{|c|c|c|c|c|c|c|c|c|c|c|c|c|}
\hline \multirow[b]{2}{*}{ Soil pit } & \multirow{2}{*}{$\begin{array}{c}\text { Sampling } \\
\text { depth } \\
\text { cm }\end{array}$} & \multicolumn{9}{|c|}{$\begin{array}{c}\text { Grain size distribution } \\
\%\end{array}$} & \multirow{2}{*}{$\begin{array}{c}\text { Granu- } \\
\text { lome- } \\
\text { tric } \\
\text { sub- } \\
\text { group }\end{array}$} & \multirow{2}{*}{$\begin{array}{c}\text { Specific } \\
\text { surface } \\
\text { area } \\
\mathrm{m}^{2} \cdot \mathrm{g}^{-1}\end{array}$} \\
\hline & & $\begin{array}{c}2.0- \\
1.0 \\
\mathrm{~mm}\end{array}$ & $\begin{array}{c}1.0- \\
0.5 \\
\mathrm{~mm}\end{array}$ & $\begin{array}{c}0.5- \\
0.25 \\
\mathrm{~mm}\end{array}$ & $\begin{array}{c}0.25- \\
0.1 \\
\mathrm{~mm}\end{array}$ & $\begin{array}{l}0.1- \\
0.05 \\
\mathrm{~mm}\end{array}$ & $\begin{array}{c}0.05- \\
0.02 \\
\mathrm{~mm}\end{array}$ & $\begin{array}{c}0.02- \\
0.005 \\
\mathrm{~mm}\end{array}$ & $\begin{array}{c}0.005- \\
0.002 \\
\mathrm{~mm}\end{array}$ & $\begin{array}{c}<0.002 \\
\mathrm{~mm}\end{array}$ & & \\
\hline Glinnik B & $0-20$ & 0.0 & 19.5 & 42.5 & 26.1 & 4.8 & 3.9 & 2.3 & 0.8 & 0.2 & $\mathrm{pl}$ & 0.0668 \\
\hline Glinnik B & $20-40$ & 0.1 & 19.4 & 42.6 & 31.6 & 4.7 & 1.6 & 0.0 & 0.0 & 0.0 & $\mathrm{pl}$ & 0.0268 \\
\hline Glinnik B & $90-110$ & 0.0 & 16.5 & 47.7 & 33.0 & 1.7 & 1.1 & 0.0 & 0.0 & 0.0 & $\mathrm{pl}$ & 0.0245 \\
\hline Glinnik B & $120-130$ & 1.3 & 12.5 & 23.3 & 22.2 & 8.0 & 8.3 & 12.0 & 7.4 & 5.1 & gp & 0.5560 \\
\hline
\end{tabular}

\begin{tabular}{|c|c|c|c|c|c|c|c|c|c|c|}
\hline \multirow[t]{2}{*}{ Soil pit } & \multirow{2}{*}{$\begin{array}{c}\text { Sampling } \\
\text { depth } \\
\text { cm }\end{array}$} & \multirow{2}{*}{$\begin{array}{c}\text { Humus } \\
\%\end{array}$} & \multirow{2}{*}{$\begin{array}{c}\text { C total } \\
\%\end{array}$} & \multirow{2}{*}{$\begin{array}{c}\mathrm{N} \text { total } \\
\%\end{array}$} & \multirow[t]{2}{*}{$\mathrm{C} / \mathrm{N}$} & \multicolumn{2}{|c|}{$\begin{array}{l}\mathrm{pH} \\
\text { in }\end{array}$} & \multicolumn{3}{|c|}{$\begin{array}{c}\text { Available nutrients } \\
\mathrm{mg} / 100 \mathrm{~g}\end{array}$} \\
\hline & & & & & & $\mathrm{KCl}$ & $\mathrm{H}_{2} \mathrm{O}$ & $\mathrm{P}_{2} \mathrm{O}_{5}$ & $\mathrm{~K}_{2} \mathrm{O}$ & $\mathrm{Mg}$ \\
\hline Glinnik B & $0-20$ & 0.86 & 0.499 & 0.036 & 13.86 & 4.2 & 5.0 & 10.6 & 1.4 & 0.4 \\
\hline Glinnik B & $20-40$ & - & - & - & - & 4.6 & 5.5 & 4.0 & 0.7 & 0.3 \\
\hline Glinnik B & $90-110$ & - & - & - & - & 4.5 & 5.4 & 3.4 & 1.8 & 0.4 \\
\hline Glinnik B & $120-130$ & - & - & - & - & 4.4 & 5.7 & 4.1 & 9.6 & 12.6 \\
\hline
\end{tabular}

\begin{tabular}{|c|c|c|c|c|c|c|c|c|c|c|}
\hline \multirow[t]{2}{*}{ Soil pit } & \multirow{2}{*}{$\begin{array}{l}\text { Sampling } \\
\text { depth } \\
\text { cm }\end{array}$} & \multirow{2}{*}{$\begin{array}{c}\text { Hydrolytic } \\
\text { acidity } \\
\text { mmol/100 g }\end{array}$} & \multicolumn{4}{|c|}{$\begin{array}{c}\text { Exchangeable cations } \\
\mathrm{me} / 100 \mathrm{~g}\end{array}$} & \multicolumn{4}{|c|}{$\begin{array}{l}\text { Sorption capacity } \\
\text { me } / 100 \mathrm{~g}\end{array}$} \\
\hline & & & $\mathrm{Ca}^{2+}$ & $\mathrm{Mg}^{2+}$ & $\mathrm{Na}^{+}$ & $\mathrm{K}^{+}$ & S & $\mathrm{T}$ & $(\mathrm{T}-\mathrm{S})$ & $\mathrm{V}, \%$ \\
\hline Glinnik B & $0-20$ & 2.65 & 0.15 & 0.033 & 0.009 & 0.044 & 0.236 & 2.886 & 2.65 & 8.2 \\
\hline Glinnik B & $20-40$ & 1.25 & 0.10 & 0.020 & 0.026 & 0.038 & 0.184 & 1.434 & 1.25 & 12.8 \\
\hline Glinnik B & $90-110$ & 1.16 & 0.05 & 0.030 & 0.009 & 0.044 & 0.133 & 1.297 & 1.16 & 10.3 \\
\hline Glinnik B & $120-130$ & 1.54 & 2.80 & 1.210 & 0.087 & 0.246 & 4.343 & 5.883 & 1.54 & 73.8 \\
\hline
\end{tabular}

Source: own elaboration.

\section{Characteristics of the flora and fungi - Glinnik B}

Approximately $90 \%$ of the study plot is covered with plants. There are fairly many species of flora (24). Three species dominate: Agrostis capillaris (about 30\%), Hieracium pilosella (about 30\%) and Jasione montana (10\%). The share of other species does not exceed $5-0.5 \%$ of the area. Grasses are a quantitatively significant component of the flora, but the number of species is small (4). Apart from Agrostis capillaris, they include: Festuca ovina, Nardus stricta and Corynephorus canescens. The observed species of dicotyledons include: Artemisia campestris, Centaurea stoebe jacobea (Tab. 6.1). The area also features a few saplings of trees: Pinus sylvestris and Quercus robur.

Fungi of the plot are rather sparse. They are represented by as few as 6 species of macromycetes. The most frequently found species is Crinipellis scabella, which occurs on grasses, mainly on Agrostis capillaris and Festuca ovina. Among land fungi, the most abundant species were Marasmius oreades and Bovista plumbea. Apart from them, rare fruit bodies of Conocybe tenera were found. Numerous fruit bodies of mycorrhizal species were identified: Suillus bovinus and Inocybe maculata. 
STUDY PLOT GLINNIK C (51ํ3'3ㄴN, 2007'064E, elevation 172 m a.s.l.)
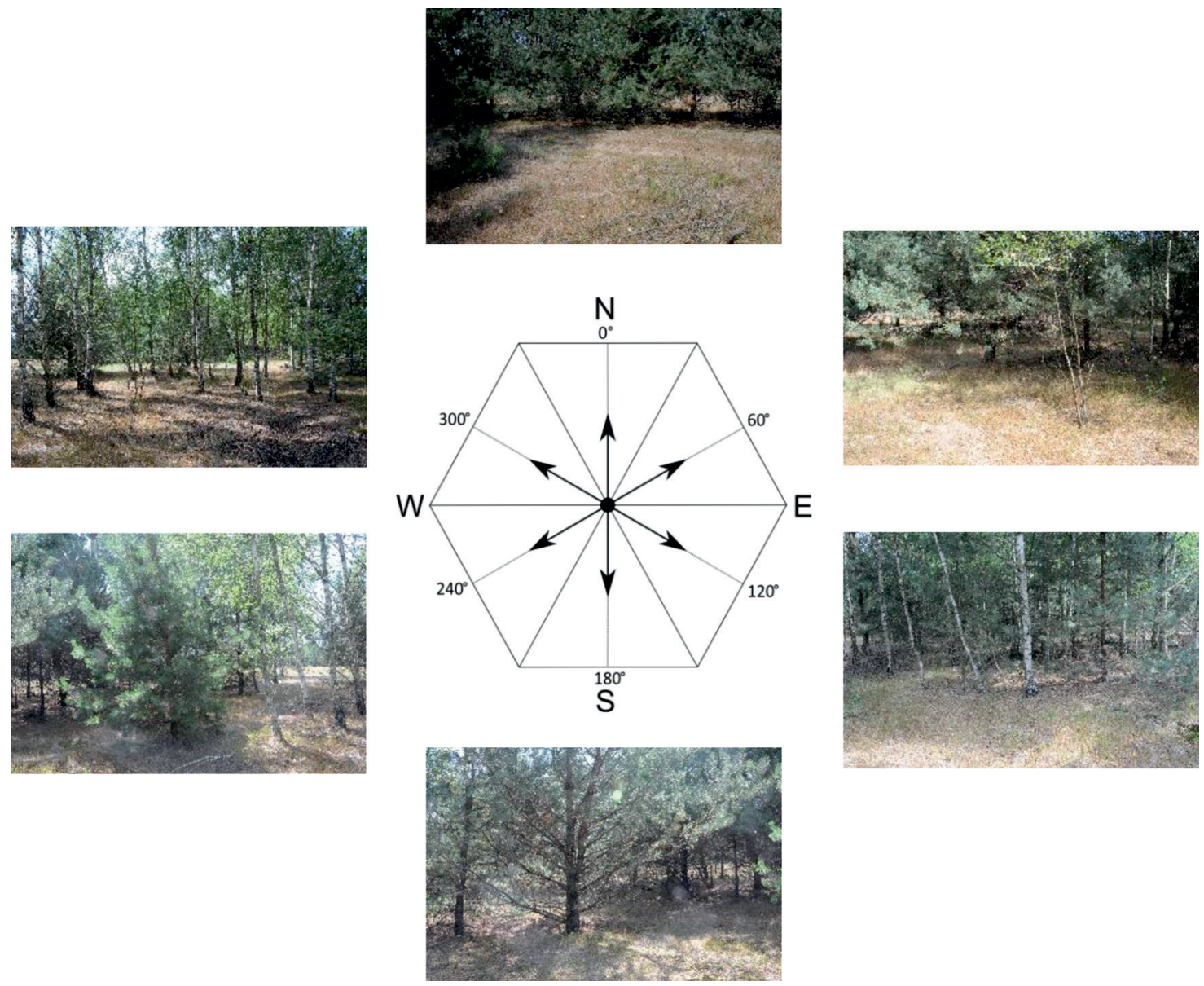

Fig. 5.5. Study plot Glinnik C (photo S. Krysiak, 2012)

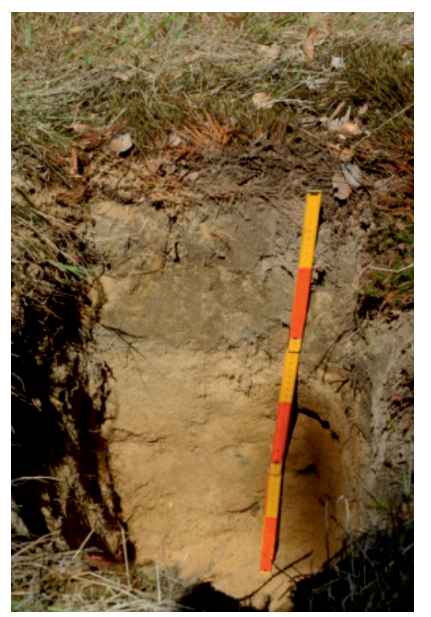

Depth Profile description

0-15 cm - humus, medium and silty sand with gravel, grey

$15-20 \mathrm{~cm}$ - medium sand, beige

20-40 cm - medium and coarse sands, yellow

40-110 cm - medium and coarse sands with single pebbles, yellow

Fig. 5.6. Soil pit in study plot Glinnik C (photo S. Krysiak, 2012) 
Table 5.3. Study plot Glinnik C. Granulometric and chemical properties of soil

\begin{tabular}{|c|c|c|c|c|c|c|c|c|c|c|c|c|}
\hline \multirow[b]{2}{*}{ Soil pit } & \multirow{2}{*}{$\begin{array}{c}\text { Sampling } \\
\text { depth } \\
\text { cm }\end{array}$} & \multicolumn{9}{|c|}{$\begin{array}{c}\text { Grain size distribution } \\
\%\end{array}$} & \multirow{2}{*}{$\begin{array}{c}\text { Granu- } \\
\text { lome- } \\
\text { tric } \\
\text { sub- } \\
\text { group }\end{array}$} & \multirow{2}{*}{$\begin{array}{c}\text { Specific } \\
\text { surface } \\
\text { area } \\
\mathrm{m}^{2} \cdot \mathrm{g}^{-1}\end{array}$} \\
\hline & & $\begin{array}{c}2.0- \\
1.0 \\
\mathrm{~mm}\end{array}$ & $\begin{array}{c}1.0- \\
0.5 \\
\mathrm{~mm}\end{array}$ & $\begin{array}{l}0.5- \\
0.25 \\
\mathrm{~mm}\end{array}$ & $\begin{array}{c}0.25- \\
0.1 \\
\mathrm{~mm}\end{array}$ & $\begin{array}{l}0.1- \\
0.05 \\
\mathrm{~mm}\end{array}$ & $\begin{array}{c}0.05- \\
0.02 \\
\mathrm{~mm}\end{array}$ & $\begin{array}{c}0.02- \\
0.005 \\
\mathrm{~mm}\end{array}$ & $\begin{array}{c}0.005- \\
0.002 \\
\mathrm{~mm}\end{array}$ & $\begin{array}{c}<0.002 \\
\mathrm{~mm}\end{array}$ & & \\
\hline Glinnik C & $0-20$ & 6.7 & 38.7 & 32.9 & 7.7 & 4.5 & 5.3 & 3.1 & 0.8 & 0.3 & $\mathrm{pl}$ & 0.0734 \\
\hline Glinnik C & $20-40$ & 0.0 & 29.8 & 57.8 & 12.4 & 0.0 & 0.0 & 0.0 & 0.0 & 0.0 & $\mathrm{pl}$ & 0.0162 \\
\hline Glinnik C & $90-110$ & 0.0 & 5.8 & 75.7 & 18.5 & 0.0 & 0.0 & 0.0 & 0.0 & 0.0 & $\mathrm{pl}$ & 0.0193 \\
\hline
\end{tabular}

\begin{tabular}{|c|c|c|c|c|c|c|c|c|c|c|}
\hline \multirow[t]{2}{*}{ Soil pit } & \multirow{2}{*}{$\begin{array}{c}\text { Sampling } \\
\text { depth } \\
\text { cm }\end{array}$} & \multirow{2}{*}{$\begin{array}{l}\text { Humus } \\
\%\end{array}$} & \multirow{2}{*}{$\begin{array}{l}\text { C total } \\
\%\end{array}$} & \multirow{2}{*}{$\begin{array}{l}\mathrm{N} \text { total } \\
\%\end{array}$} & \multirow[t]{2}{*}{$\mathrm{C} / \mathrm{N}$} & \multicolumn{2}{|c|}{$\begin{array}{l}\mathrm{pH} \\
\text { in }\end{array}$} & \multicolumn{3}{|c|}{$\begin{array}{c}\text { Available nutrients } \\
\mathrm{mg} / 100 \mathrm{~g}\end{array}$} \\
\hline & & & & & & $\mathrm{KCl}$ & $\mathrm{H}_{2} \mathrm{O}$ & $\mathrm{P}_{2} \mathrm{O}_{5}$ & $\mathrm{~K}_{2} \mathrm{O}$ & $\mathrm{Mg}$ \\
\hline Glinnik C & $0-20$ & 1.41 & 0.818 & 0.048 & 17.04 & 4.0 & 4.7 & 10.6 & 2.3 & 0.5 \\
\hline Glinnik C & $20-40$ & - & - & - & - & 4.5 & 5.4 & 2.8 & 0.3 & 0.3 \\
\hline Glinnik C & $90-110$ & - & - & - & - & 4.7 & 5.7 & 1.3 & 0.3 & 0.2 \\
\hline
\end{tabular}

\begin{tabular}{|c|c|c|c|c|c|c|c|c|c|c|}
\hline & \multirow{2}{*}{ Soil pit } & \multirow{2}{*}{$\begin{array}{c}\text { Sampling } \\
\text { depth } \\
\mathrm{cm}\end{array}$} & \multirow{2}{*}{$\begin{array}{c}\text { Hydrolytic } \\
\text { acidity } \\
\mathrm{mmol} / 100 \mathrm{~g}\end{array}$} & \multicolumn{4}{|c|}{$\begin{array}{c}\text { Exchangeable cations } \\
\mathrm{me} / 100 \mathrm{~g}\end{array}$} & \multicolumn{4}{c|}{$\begin{array}{c}\text { Sorption capacity } \\
\mathrm{me} / 100 \mathrm{~g}\end{array}$} \\
\cline { 5 - 14 } & & $\mathrm{Ca}^{2+}$ & $\mathrm{Mg}^{2+}$ & $\mathrm{Na}^{+}$ & $\mathrm{K}^{+}$ & $\mathrm{S}$ & $\mathrm{T}$ & $(\mathrm{T}-\mathrm{S})$ & $\mathrm{V}, \%$ \\
\hline Glinnik C & $0-20$ & 1.48 & 0.10 & 0.033 & 0.009 & 0.074 & 0.155 & 1.635 & 1.48 & 9.5 \\
\hline Glinnik C & $20-40$ & 1.01 & 0.05 & 0.015 & 0.009 & 0.013 & 0.087 & 1.097 & 1.01 & 7.9 \\
\hline Glinnik C & $90-110$ & 0.65 & $\mathrm{ND}$ & 0.117 & $\mathrm{ND}$ & 0.005 & 0.122 & 0.772 & 0.65 & 15.8 \\
\hline
\end{tabular}

Source: own elaboration.

\section{Characteristics of the flora and fungi - Glinnik C}

Approximately $100 \%$ of the study plot is covered with plants. Poor in species of vascular plants (8). One species dominates: Agrostis capillaris, covering about $80 \%$ of the area. A much lower share (7-3\%) occurs of e.g.: Apera spica-venti, Hieracium pilosella (Tab. 6.1) and saplings of trees: Pinus sylvestris, Quercus robur and Betula pendula. The occurrence of three species of moss was also identified: Polytrichum attenuatum, Pleurozium schreberii and Dicranum scoparium, which covered a small portion of the area (about 2\%).

Fungi of the plot are represented by 9 species of macromycetes. None of the species was abundant. The identified species include: Bovista nigrescens, Calocybe gambosa, Marasmius oreades and others (Tab. 7.1).

\section{Analysis of granulometric composition and chemical properties of soils in the Glinnik study plot group}

Granulometric analyses of study plots Glinnik A, $B$ and C (Tab. 5.1-5.3) reveal a slight diversity. The dominating role is played by the sandy fraction. At Glinnik A, about $15 \%$ share of the silty fraction allowed the surface series to be qualified as the granulometric subgroup of slightly loamy sands (ps). Underneath them lie loose sands (pl), completely devoid of silts. Also at Glinnik B (sample from the depths: 0-20 and 20-40 cm) and Glinnik C (sample from the depth of $0-20 \mathrm{~cm}$ ), the content of several percent of silts is present, confirmed with larger values of specific surface area. As regards agronomic classification, the granulometric composition of the analysed sediments qualify them as soils of category I (very light soils). The sandy loam found at Glinnik B is an exception, classified as category III (medium soils).

The $\mathrm{pH}$ reaction in $\mathrm{KCl}$ for all samples from the three sites is acidic and very acidic. The highest reaction spread was recorded at Glinnik C - from 
4.0 to 4.7. The available content of phosphorus $\left(\mathrm{P}_{2} \mathrm{O}_{5}\right)$, potassium $\left(\mathrm{K}_{2} \mathrm{O}\right)$ and magnesium $(\mathrm{Mg})$ at all analysed sites corresponds to very low and low class of availability. The content of alkaline cations $\mathrm{Ca}^{2+}, \mathrm{Mg}^{2+}, \mathrm{Na}^{+}$and $\mathrm{K}^{+}$is also low, which confirms the low saturation of the sorptive complex (V), ranging from several to about a dozen percent. The sandy loam from Glinnik B is an exception here, with saturation of the above mentioned cations reaching 73.8\%.

\section{CELESTYNÓW STUDY PLOT GROUP}

The Celestynów study plot group is located $3 \mathrm{~km}$ away from the Sulejów Landscape Park and $8 \mathrm{~km}$ away from the borders of the Spała Landscape Park, in Sławno Commune. It is a part of a vast stretch of land belonging to the villages of Unewel and Celestynów, where most croplands were excluded from farming and became abandoned, representing various degrees of secondary succession. In the west and south the abandoned lands border directly on woodlands. There are also extensive woodlands not far away to the north and east of the study area. Surface deposits in the Celestynów study plot group include sands and fluvioglacial gravels, which originated in the period of the Odranian Glaciation (Szałama- cha 1991). A slight admixture of the silty fraction, found in the uppermost series of plots Celestynów $\mathrm{A}, \mathrm{B}$ and $\mathrm{C}$, is related to aeolian accumulation in the periglacial conditions of the Wartanian and Vistulian. The deposits constitute the parent rock for sandy soils of different genetic types (AB), whose agricultural suitability was classified as very poor rye complex (7) (Województwo piotrkowskie. Mapa glebowo-rolnicza. 1979). As regards soil valuation, productivity of soils at all plots of the Celestynów group was qualified as class VI (http:// geoportal.lodzkie.pl/imap/). Photographic documentation and results of laboratory analyses from the plots of the Celestynów study plot group are presented in figures 5.7-5.12, and in tables 5.4-5.6.

STUDY PLOT CELESTYNÓW A (5126'175N, 2004'494E, elevation 205 m a.s.l.)
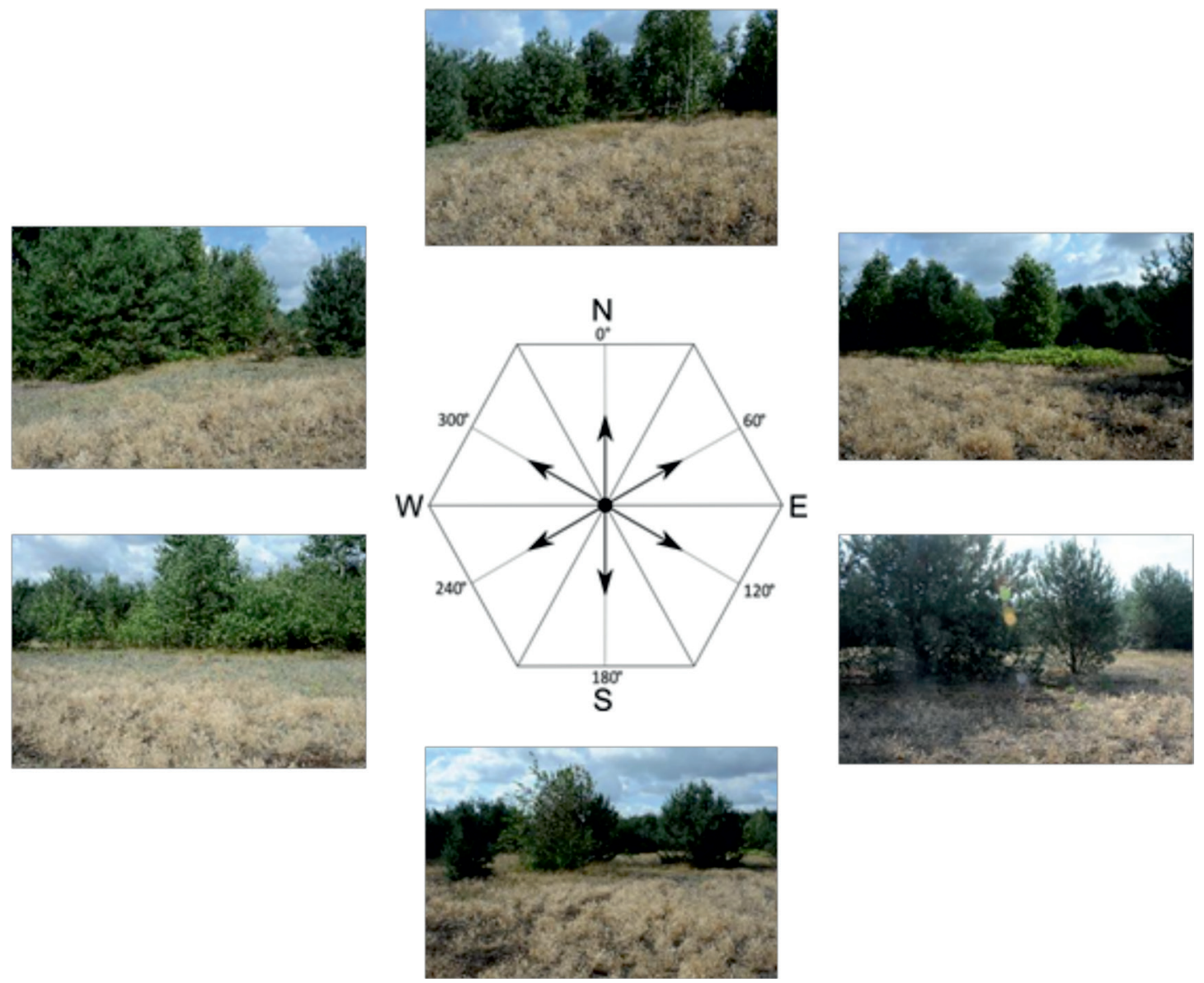

Fig. 5.7. Study plot Celestynów A (photo S. Krysiak, 2012) 


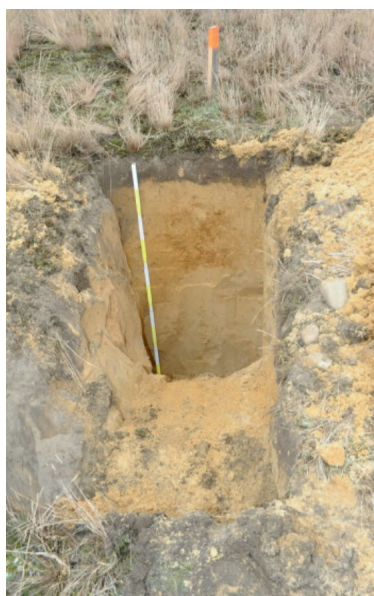

\author{
Depth Profile description \\ 0-15 cm - humus, fine and medium sand with gravel and cobbles \\ of up to $4 \mathrm{~cm}$ in diameter, grey \\ $15-40 \mathrm{~cm}$ - medium and coarse sand with gravel, rusty yellow \\ 40-60 cm - medium and coarse sand with single pebbles, rusty \\ $60-110 \mathrm{~cm}$ - fine and medium sand with single pebbles, yellow
}

Fig. 5.8. Soil pit in study plot Celestynów A (photo S. Krysiak, 2012)

Table 5.4. Study plot Celestynów A. Granulometric and chemical properties of soil

\begin{tabular}{|c|c|c|c|c|c|c|c|c|c|c|c|c|}
\hline \multirow[b]{2}{*}{ Soil pit } & \multirow{2}{*}{$\begin{array}{c}\text { Sampling } \\
\text { depth } \\
\text { cm }\end{array}$} & \multicolumn{9}{|c|}{$\begin{array}{c}\text { Grain size distribution } \\
\%\end{array}$} & \multirow{2}{*}{$\begin{array}{l}\text { Gra- } \\
\text { nulo- } \\
\text { metric } \\
\text { sub- } \\
\text { group }\end{array}$} & \multirow{2}{*}{$\begin{array}{c}\text { Spe- } \\
\text { cific } \\
\text { surface } \\
\text { area } \\
\mathrm{m}^{2} \cdot \mathrm{g}^{-1}\end{array}$} \\
\hline & & $\begin{array}{c}2.0- \\
1.0 \\
\mathrm{~mm}\end{array}$ & $\begin{array}{c}1.0- \\
0.5 \\
\mathrm{~mm}\end{array}$ & $\begin{array}{l}0.5- \\
0.25 \\
\mathrm{~mm}\end{array}$ & $\begin{array}{c}0.25- \\
0.1 \\
\mathrm{~mm}\end{array}$ & $\begin{array}{l}0.1- \\
0.05 \\
\mathrm{~mm}\end{array}$ & $\begin{array}{c}0.05- \\
0.02 \\
\mathrm{~mm}\end{array}$ & $\begin{array}{c}0.02- \\
0.005 \\
\mathrm{~mm}\end{array}$ & $\begin{array}{c}0.005- \\
0.002 \\
\mathrm{~mm}\end{array}$ & $\begin{array}{c}<0.002 \\
\mathrm{~mm}\end{array}$ & & \\
\hline Celestynów A & $0-20$ & 5.2 & 38.3 & 37.5 & 10.4 & 2.2 & 2.7 & 2.7 & 0.9 & 0.1 & $\mathrm{pl}$ & 0.0577 \\
\hline Celestynów A & $20-40$ & 7.3 & 40.3 & 37.9 & 8.3 & 0.7 & 1.6 & 2.4 & 1.1 & 0.4 & $\mathrm{pl}$ & 0.0685 \\
\hline Celestynów A & $90-110$ & 4.3 & 38.1 & 46.3 & 11.4 & 0.0 & 0.0 & 0.0 & 0.0 & 0.0 & $\mathrm{pl}$ & 0.0148 \\
\hline
\end{tabular}

\begin{tabular}{|c|c|c|c|c|c|c|c|c|c|c|}
\hline \multirow{2}{*}{ Soil pit } & \multirow{2}{*}{$\begin{array}{c}\text { Sampling } \\
\text { depth } \\
\text { cm }\end{array}$} & \multirow{2}{*}{$\begin{array}{c}\text { Humus } \\
\%\end{array}$} & \multirow{2}{*}{$\begin{array}{c}\text { C total } \\
\%\end{array}$} & \multirow{2}{*}{$\begin{array}{c}\mathrm{N} \text { total } \\
\%\end{array}$} & \multirow{2}{*}{$\mathrm{C} / \mathrm{N}$} & \multicolumn{2}{|c|}{$\begin{array}{l}\mathrm{pH} \\
\text { in }\end{array}$} & \multicolumn{3}{|c|}{$\begin{array}{c}\text { Available nutrients } \\
\mathrm{mg} / 100 \mathrm{~g}\end{array}$} \\
\hline & & & & & & $\mathrm{KCl}$ & $\mathrm{H}_{2} \mathrm{O}$ & $\mathrm{P}_{2} \mathrm{O}_{5}$ & $\mathrm{~K}_{2} \mathrm{O}$ & $\mathrm{Mg}$ \\
\hline Celestynów A & $0-20$ & 1.37 & 0.795 & 0.047 & 16.91 & 4.0 & 4.3 & 2.6 & 0.6 & 0.4 \\
\hline Celestynów A & $20-40$ & - & - & - & - & 4.2 & 4.6 & 1.5 & 0.8 & 0.3 \\
\hline Celestynów A & $90-110$ & - & - & - & - & 4.3 & 5.0 & 1.2 & 0.3 & 0.2 \\
\hline
\end{tabular}

\begin{tabular}{|c|c|c|c|c|c|c|c|c|c|c|}
\hline & \multirow{2}{*}{ Soil pit } & \multirow{2}{*}{$\begin{array}{c}\text { Sampling } \\
\text { depth } \\
\mathrm{cm}\end{array}$} & \multirow{2}{*}{$\begin{array}{c}\text { Hydrolytic } \\
\text { acidity } \\
\mathrm{mmol} / 100 \mathrm{~g}\end{array}$} & \multicolumn{4}{|c|}{$\begin{array}{c}\text { Exchangeable cations } \\
\mathrm{me} / 100 \mathrm{~g}\end{array}$} & \multicolumn{4}{c|}{$\begin{array}{c}\text { Sorption capacity } \\
\mathrm{me} / 100 \mathrm{~g}\end{array}$} \\
\cline { 5 - 13 } & & $\mathrm{Ca}^{2+}$ & $\mathrm{Mg}^{2+}$ & $\mathrm{Na}^{+}$ & $\mathrm{K}^{+}$ & $\mathrm{S}$ & $\mathrm{T}$ & $(\mathrm{T}-\mathrm{S})$ & $\mathrm{V}, \%$ \\
\hline Celestynów A & $0-20$ & 3.65 & $\mathrm{ND}$ & 0.020 & 0.009 & 0.026 & 0.055 & 3.705 & 3.65 & 1.5 \\
\hline Celestynów A & $20-40$ & 2.43 & $\mathrm{ND}$ & 0.020 & 0.078 & 0.026 & 0.124 & 2.554 & 2.43 & 4.9 \\
\hline Celestynów A & $90-110$ & 1.14 & $\mathrm{ND}$ & 0.015 & 0.009 & 0.005 & 0.029 & 1.169 & 1.14 & 2.5 \\
\hline
\end{tabular}

Source: own elaboration. 


\section{Characteristics of the flora and fungi - Celestynów A}

Approximately $100 \%$ of the study plot is covered with plants. Poor in species of vascular plants (10). One species dominates: Corynephorus canescens, covering about $70 \%$ of the area. A much lower share (3-0.5\%) occurs of such species as: Agrostis capillaris, Hieracium pilosella, Rumex acetosella, Solidago canadensis and saplings of trees: Pinus sylvestris, Quercus robur and Padus serotina
(Tab. 6.1). One species of moss was also identified: Polytrichum piliferum, which covered a small portion of the area (about 1\%).

Fungi of the plot are not numerous. They are represented by 11 species of macromycetes. None of them was abundant. The identified species include: Amanita muscaria, Bovista plumbea, Lycoperdon nigrescens and others (Tab. 7.1).

\section{STUDY PLOT CELESTYNÓW B (51206'193N, 2004'463E, elevation 206 m a.s.l.)}
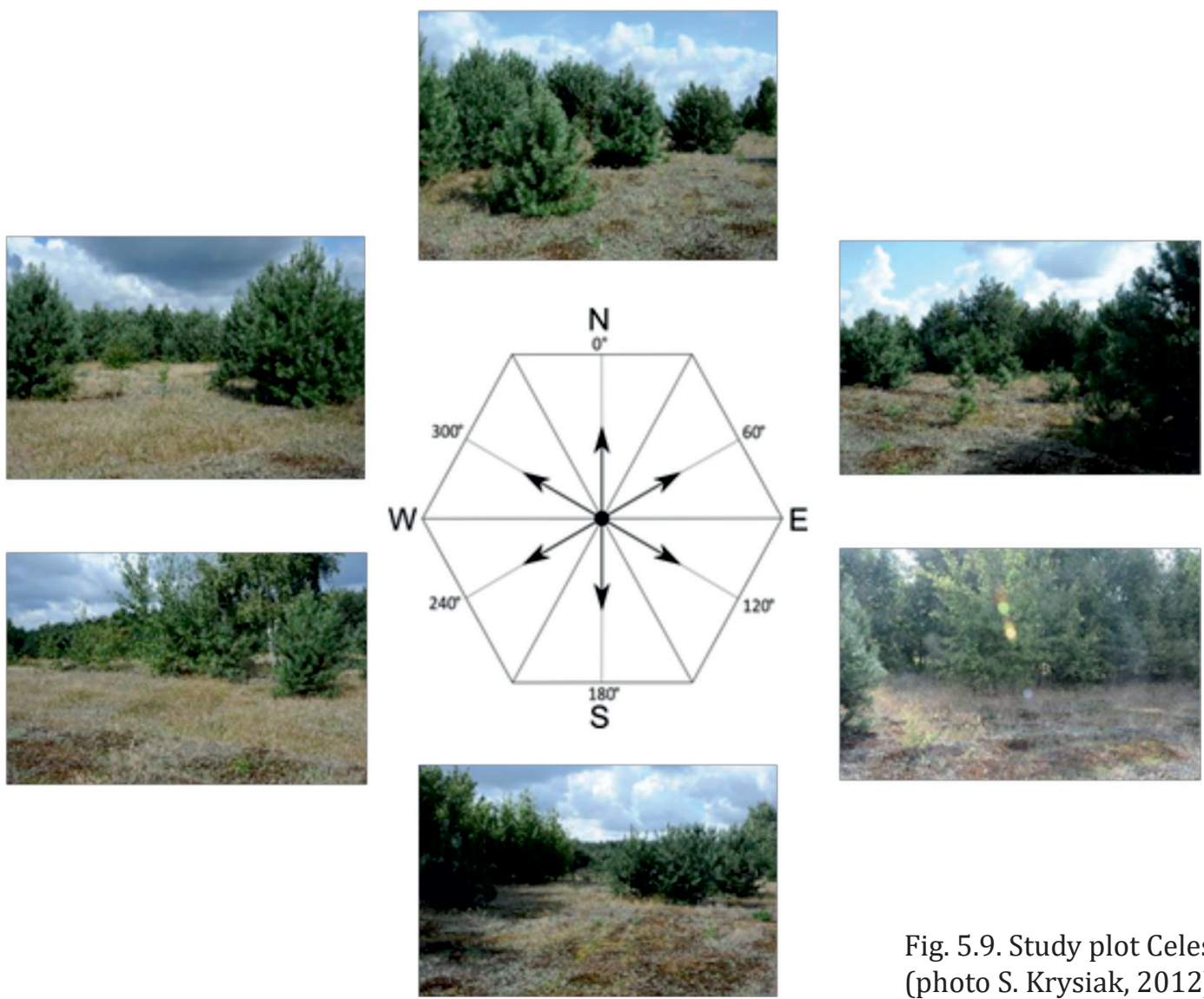

Fig. 5.9. Study plot Celestynów B (photo S. Krysiak, 2012)

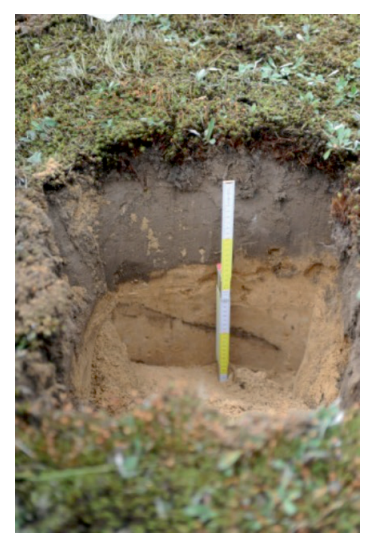

\footnotetext{
Depth Profile description

$0-20 \mathrm{~cm}$ - humus, fine and silty sand, grey

20-40 cm - medium and fine sand with cobbles of Scandinavian origin, grey

40-70 cm - medium sand with cobbles on the bottom, yellow

$70-110 \mathrm{~cm}$ - medium sand with gravel, rusty
}

Fig. 5.10. Soil pit in study plot Celestynów B (photo S. Krysiak, 2012) 
Table 5.5. Study plot Celestynów B. Granulometric and chemical properties of soil

\begin{tabular}{|c|c|c|c|c|c|c|c|c|c|c|c|c|}
\hline \multirow[b]{2}{*}{ Soil pit } & \multirow{2}{*}{$\begin{array}{c}\text { Sampling } \\
\text { depth } \\
\text { cm }\end{array}$} & \multicolumn{9}{|c|}{$\begin{array}{c}\text { Grain size distribution } \\
\%\end{array}$} & \multirow{2}{*}{$\begin{array}{c}\text { Granu- } \\
\text { lome- } \\
\text { tric } \\
\text { sub- } \\
\text { group }\end{array}$} & \multirow{2}{*}{$\begin{array}{l}\text { Spe- } \\
\text { cific } \\
\text { surface } \\
\text { area } \\
\mathrm{m}^{2} \cdot \mathrm{g}^{-1}\end{array}$} \\
\hline & & $\begin{array}{c}2.0- \\
1.0 \\
\mathrm{~mm}\end{array}$ & $\begin{array}{c}1.0- \\
0.5 \\
\mathrm{~mm}\end{array}$ & $\begin{array}{l}0.5- \\
0.25 \\
\mathrm{~mm}\end{array}$ & $\begin{array}{c}0.25- \\
0.1 \\
\mathrm{~mm}\end{array}$ & $\begin{array}{l}0.1- \\
0.05 \\
\mathrm{~mm}\end{array}$ & $\begin{array}{c}0.05- \\
0.02 \\
\mathrm{~mm}\end{array}$ & $\begin{array}{c}0.02- \\
0.005 \\
\mathrm{~mm}\end{array}$ & $\begin{array}{c}0.005- \\
0.002 \\
\mathrm{~mm}\end{array}$ & $\begin{array}{c}<0.002 \\
\mathrm{~mm}\end{array}$ & & \\
\hline Celestynów B & $0-20$ & 2.3 & 27.7 & 37.6 & 19.0 & 4.3 & 4.0 & 3.4 & 1.2 & 0.6 & $\mathrm{pl}$ & 0.1000 \\
\hline Celestynów B & $20-40$ & 1.6 & 27.3 & 41.7 & 19.5 & 2.0 & 2.3 & 3.1 & 1.6 & 0.9 & $\mathrm{pl}$ & 0.1170 \\
\hline Celestynów B & $90-110$ & 0.1 & 31.7 & 54.1 & 9.0 & 0.5 & 0.9 & 1.9 & 1.2 & 0.5 & $\mathrm{pl}$ & 0.0763 \\
\hline
\end{tabular}

\begin{tabular}{|c|c|c|c|c|c|c|c|c|c|c|}
\hline \multirow[t]{2}{*}{ Soil pit } & \multirow{2}{*}{$\begin{array}{c}\text { Sampling } \\
\text { depth } \\
\mathrm{cm}\end{array}$} & \multirow{2}{*}{$\begin{array}{c}\text { Humus } \\
\%\end{array}$} & \multirow{2}{*}{$\begin{array}{c}\mathrm{C} \text { total } \\
\%\end{array}$} & \multirow{2}{*}{$\begin{array}{c}\mathrm{N} \text { total } \\
\%\end{array}$} & \multirow[t]{2}{*}{$\mathrm{C} / \mathrm{N}$} & \multicolumn{2}{|c|}{$\begin{array}{l}\mathrm{pH} \\
\text { in }\end{array}$} & \multicolumn{3}{|c|}{$\begin{array}{l}\text { Available nutrients } \\
\mathrm{mg} / 100 \mathrm{~g}\end{array}$} \\
\hline & & & & & & $\mathrm{KCl}$ & $\mathrm{H}_{2} \mathrm{O}$ & $\mathrm{P}_{2} \mathrm{O}_{5}$ & $\mathrm{~K}_{2} \mathrm{O}$ & $\mathrm{Mg}$ \\
\hline Celestynów B & $0-20$ & 1.42 & 0.824 & 0.051 & 16.16 & 4.1 & 4.6 & 2.2 & 0.3 & 0.3 \\
\hline Celestynów B & $20-40$ & - & - & - & - & 4.5 & 4.8 & 2.7 & 0.3 & 0.2 \\
\hline Celestynów B & $90-110$ & - & - & - & - & 4.2 & 4.6 & 1.0 & 0.6 & 0.3 \\
\hline
\end{tabular}

\begin{tabular}{|c|c|c|c|c|c|c|c|c|c|c|}
\hline \multirow[t]{2}{*}{ Soil pit } & \multirow{2}{*}{$\begin{array}{c}\text { Sampling } \\
\text { depth } \\
\mathrm{cm}\end{array}$} & \multirow{2}{*}{$\begin{array}{c}\text { Hydrolytic } \\
\text { acidity } \\
\text { mmol } / 100 \mathrm{~g}\end{array}$} & \multicolumn{4}{|c|}{$\begin{array}{c}\text { Exchangeable cations } \\
\text { me } / 100 \mathrm{~g}\end{array}$} & \multicolumn{4}{|c|}{$\begin{array}{c}\text { Sorption capacity } \\
\text { me/100 g }\end{array}$} \\
\hline & & & $\mathrm{Ca}^{2+}$ & $\mathrm{Mg}^{2+}$ & $\mathrm{Na}^{+}$ & $\mathrm{K}^{+}$ & $\mathrm{S}$ & $\mathrm{T}$ & (T-S) & $\mathrm{V}, \%$ \\
\hline Celestynów B & $0-20$ & 3.80 & 0.10 & 0.018 & 0.017 & 0.018 & 0.153 & 3.953 & 3.80 & 3.9 \\
\hline Celestynów B & $20-40$ & 1.52 & 0.10 & 0.013 & 0.009 & 0.013 & 0.135 & 1.655 & 1.52 & 8.2 \\
\hline Celestynów B & $90-110$ & 1.92 & 0.10 & 0.015 & 0.009 & 0.013 & 0.137 & 2.057 & 1.92 & 6.7 \\
\hline
\end{tabular}

Source: own elaboration.

\section{Characteristics of the flora and fungi - Celestynów B}

Approximately $70 \%$ of the plot area is covered with plants. Poor in species of vascular plants (8). One species dominates: Hieracium pilosella, covering about $50 \%$ of the area. Species occurring with a lower share: Agrostis capillaris (about $10 \%$ ) and Jasione montana (about 3\%). They are accompanied in slight quantities by e.g.: Achillea millefilium, Anthoxathum odoratum, Holcus mollis
(Tab. 6.1). The occurrence of one species of moss was also identified: Polytrichum piliferum, which covered a large portion of the area (about 70\%).

Fungi of the plot are sparse. They are represented by 7 species of macromycetes, of which the only abundant species were gasteroid fungi, e.g. Calvatia excipuliformis, Vascellum pratense (Tab. 7.1). 
STUDY PLOT CELESTYNóW C (51ํ26'138N, 2004'547E, elevation 206 m a.s.l.)
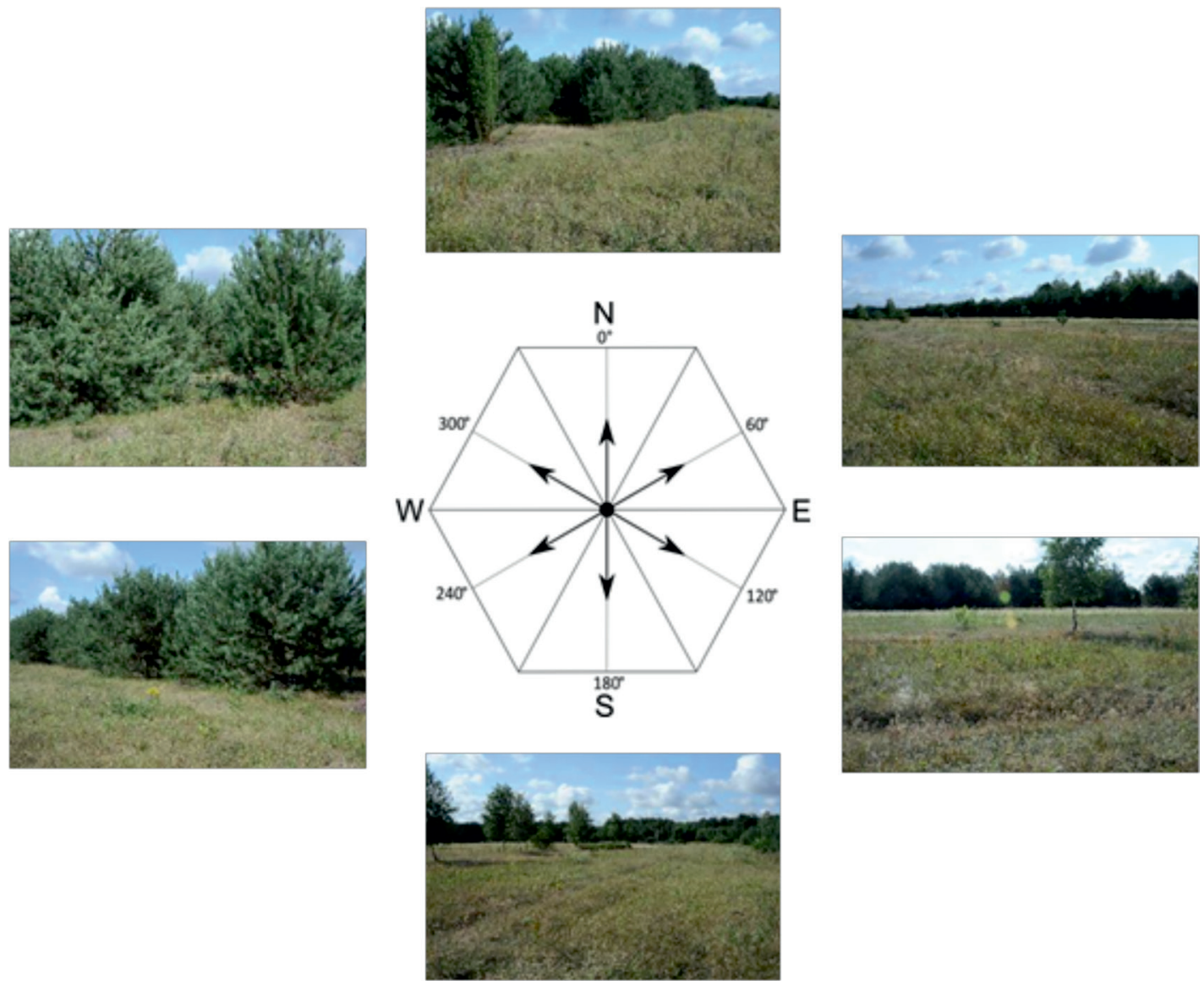

Fig. 5.11. Study plot Celestynów C (photo S. Krysiak, 2012)

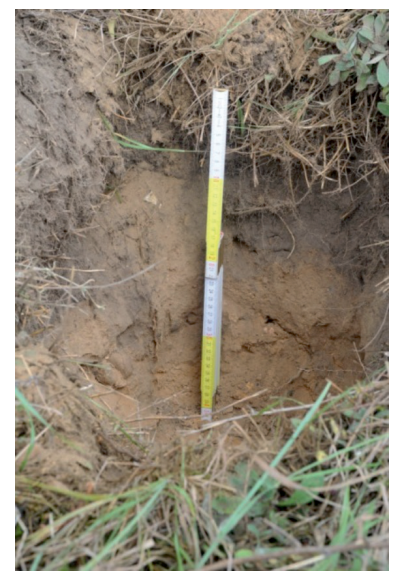

Depth Profile description

$0-20 \mathrm{~cm}$ - humus, fine and silty sand with cobbles, grey

20-45 cm - medium and fine sand with numerous cobbles of Scandinavian origin with diameters of up to $10 \mathrm{~cm}$, greyish yellow

45-70 cm - medium and fine sands, light beige

$70-110 \mathrm{~cm}$ - fine and medium sands with single pebbles, light beige

Fig. 5.12. Soil pit in study plot Celestynów C (photo S. Krysiak, 2012) 
Table 5.6. Study plot Celestynów C. Granulometric and chemical properties of soil

\begin{tabular}{|c|c|c|c|c|c|c|c|c|c|c|c|c|}
\hline \multirow[b]{2}{*}{ Soil pit } & \multirow{2}{*}{$\begin{array}{c}\text { Sampling } \\
\text { depth } \\
\mathrm{cm}\end{array}$} & \multicolumn{9}{|c|}{$\begin{array}{c}\text { Grain size distribution } \\
\%\end{array}$} & \multirow{2}{*}{$\begin{array}{c}\text { Granu- } \\
\text { lome- } \\
\text { tric } \\
\text { sub- } \\
\text { group }\end{array}$} & \multirow{2}{*}{$\begin{array}{c}\text { Spe- } \\
\text { cific } \\
\text { surface } \\
\text { area } \\
\mathrm{m}^{2} \cdot \mathrm{g}^{-1}\end{array}$} \\
\hline & & $\begin{array}{c}2.0- \\
1.0 \\
\mathrm{~mm}\end{array}$ & $\begin{array}{c}1.0- \\
0.5 \\
\mathrm{~mm}\end{array}$ & $\begin{array}{l}0.5- \\
0.25 \\
\mathrm{~mm}\end{array}$ & $\begin{array}{c}0.25- \\
0.1 \\
\mathrm{~mm}\end{array}$ & $\begin{array}{l}0.1- \\
0.05 \\
\mathrm{~mm}\end{array}$ & $\begin{array}{c}0.05- \\
0.02 \\
\mathrm{~mm}\end{array}$ & $\begin{array}{c}0.02- \\
0.005 \\
\mathrm{~mm}\end{array}$ & $\begin{array}{c}0.005- \\
0.002 \\
\mathrm{~mm}\end{array}$ & $\begin{array}{c}<0.002 \\
\mathrm{~mm}\end{array}$ & & \\
\hline Celestynów C & $0-20$ & 1.6 & 25.2 & 36.6 & 19.2 & 6.5 & 5.9 & 3.5 & 1.0 & 0.5 & $\mathrm{pl}$ & 0.0966 \\
\hline Celestynów C & $20-40$ & 2.8 & 26.2 & 37.2 & 21.0 & 4.0 & 3.4 & 3.1 & 1.3 & 0.9 & pl & 0.1200 \\
\hline Celestynów C & $90-110$ & 4.4 & 28.8 & 42.1 & 17.6 & 0.3 & 1.9 & 2.5 & 1.6 & 0.8 & $\mathrm{pl}$ & 0.1070 \\
\hline
\end{tabular}

\begin{tabular}{|c|c|c|c|c|c|c|c|c|c|c|}
\hline \multirow[t]{2}{*}{ Soil pit } & \multirow{2}{*}{$\begin{array}{c}\text { Sampling } \\
\text { depth } \\
\mathrm{cm}\end{array}$} & \multirow{2}{*}{$\begin{array}{c}\text { Humus } \\
\%\end{array}$} & \multirow{2}{*}{$\begin{array}{c}\text { C total } \\
\%\end{array}$} & \multirow{2}{*}{$\begin{array}{l}\mathrm{N} \text { total } \\
\%\end{array}$} & \multirow[t]{2}{*}{$\mathrm{C} / \mathrm{N}$} & \multicolumn{2}{|c|}{$\begin{array}{l}\mathrm{pH} \\
\text { in }\end{array}$} & \multicolumn{3}{|c|}{$\begin{array}{c}\text { Available nutrients } \\
\mathrm{mg} / 100 \mathrm{~g}\end{array}$} \\
\hline & & & & & & $\mathrm{KCl}$ & $\mathrm{H}_{2} \mathrm{O}$ & $\mathrm{P}_{2} \mathrm{O}_{5}$ & $\mathrm{~K}_{2} \mathrm{O}$ & $\mathrm{Mg}$ \\
\hline Celestynów C & $0-20$ & 1.77 & 1.027 & 0.076 & 13.51 & 5.5 & 6.3 & 14.3 & 1.3 & 2.2 \\
\hline Celestynów C & $20-40$ & - & - & - & - & 5.1 & 6.3 & 2.5 & 2.1 & 1.5 \\
\hline Celestynów C & $90-110$ & - & - & - & - & 4.3 & 5.3 & 1.3 & 1.1 & 1.8 \\
\hline
\end{tabular}

\begin{tabular}{|c|c|c|c|c|c|c|c|c|c|c|}
\hline \multirow[t]{2}{*}{ Soil pit } & \multirow{2}{*}{$\begin{array}{c}\text { Sampling } \\
\text { depth } \\
\mathrm{cm}\end{array}$} & \multirow{2}{*}{$\begin{array}{l}\text { Hydrolytic } \\
\text { acidity } \\
\text { mmol/100 g }\end{array}$} & \multicolumn{4}{|c|}{$\begin{array}{c}\text { Exchangeable cations } \\
\mathrm{me} / 100 \mathrm{~g}\end{array}$} & \multicolumn{4}{|c|}{$\begin{array}{c}\text { Sorption capacity } \\
\mathrm{me} / 100 \mathrm{~g}\end{array}$} \\
\hline & & & $\mathrm{Ca}^{2+}$ & $\mathrm{Mg}^{2+}$ & $\mathrm{Na}^{+}$ & $\mathrm{K}^{+}$ & S & $\mathrm{T}$ & $(\mathrm{T}-\mathrm{S})$ & $\mathrm{V}, \%$ \\
\hline Celestynów C & $0-20$ & 1.90 & 2.60 & 0.200 & 0.052 & 0.044 & 2.896 & 4.796 & 1.90 & 60.4 \\
\hline Celestynów C & $20-40$ & 1.46 & 1.10 & 0.123 & 0.026 & 0.062 & 1.311 & 2.771 & 1.46 & 47.3 \\
\hline Celestynów C & $90-110$ & 1.18 & 0.50 & 0.152 & 0.009 & 0.031 & 0.692 & 1.872 & 1.18 & 37.0 \\
\hline
\end{tabular}

Source: own elaboration.

\section{Characteristics of the flora and fungi - Celestynów C}

Approximately $100 \%$ of the study plot is covered with plants. A large number of plant species occur here (26). Four species dominate: Elymus repens (about 15\%), Artemisia campestris (about 15\%), Agrostis capillaris (about 10\%) and Hieracium pilosella (about 10\%). Species with a larger coverage (5-7\%) include: Artemisia vulgaris, Corynepho- rus canescens, Festuca ovina, F. rubra. The share of other species does not exceed $2-0.5 \%$ of the area coverage (Tab. 6.1).

Fungi of the plot are abundant. 18 species of macromycetes were identified, of which the most frequently fruiting ones were: Conocybe tenera, Leccinum scabrum and Suillus bovinus (Tab. 7.1).

\section{Analysis of granulometric composition and chemical properties of soils in the Celestynów study plot group}

The sandy fraction dominates the three analysed plots - Celestynów A, B and C. All the analysed samples were qualified granulometrically with the subgroup of loose sands (pl) with low values of specific surface area, ranging from 0.12 to $0.0148 \mathrm{~m}^{2} \mathrm{~g}^{-1}$. At all sites, the $0-20 \mathrm{~cm}$ deep series had a slightly larger share of the silty fraction than the lower horizon. The increased share of silt indicates the periglacial origin of the $0-20 \mathrm{~cm}$ series. The minimum value of specific surface area $0.0148 \mathrm{~m}^{2} \mathrm{~g}^{-1}$, was found in a sample taken at plot
Celestynów A at the depth of 90-110 cm, and was completely devoid of the silty and clayey fractions. Lack of these fractions, or their scantiness at the depth of 90-110 cm at plot Celestynów B confirms the fluvioglacial character of the underlying series. Sediments at plot Celestynów C, despite their qualification as the granulometric subgroup of loose sand, reveal slightly more favourable agrophysical properties due to a larger share of the silty and clayey fractions. 
As regards agronomic classification, the granulometric composition of the analysed sediments qualify them as soils of category I (very light soils). The permeable character of fluvioglacial deposits results in the occurrence of the precipitation-retention type of water balance with the possibility of frequent shortages of moisture in the soil.

The $\mathrm{pH}$ reaction in $\mathrm{KCl}$ and $\mathrm{H}_{2} \mathrm{O}$ at sites Celestynów A and B is very acidic. At the same time, sediments from these sites show a very low con- tent of available phosphorus $\left(\mathrm{P}_{2} \mathrm{O}_{5}\right)$, potassium $\left(\mathrm{K}_{2} \mathrm{O}\right)$ and magnesium $(\mathrm{Mg})$ with insignificant presence of alkaline cations. The more favourable agrochemical properties of the sediments at site Celestynów $\mathrm{C}$ are confirmed by a higher reaction, a higher content of available phosphorus, potassium and magnesium and a larger share of alkaline cations $\mathrm{Ca}^{2+}, \mathrm{Mg}^{2+}, \mathrm{Na}^{+}$and $\mathrm{K}^{+}$, expressed by a high degree of saturation of the sorptive complex (V).

\section{SULEJÓW STUDY PLOT GROUP}

The Sulejów study plot group is located several hundred metres from the borders of the Sulejów Landscape Park, within the limits of Sulejów. It lies within a belt of land surrounded by forests. Currently, inside areas which were once used agriculturally, there are abandoned lands at different stages of secondary succession and strips of young stands of pine and birch. According to the Detailed Geological Map of Poland, sheet Sulejów (702) (Brzeziński 1990), the surface sediments at site Sulejów A should be aeolian sands upon periglacial sands and muds, whereas at sites Sulejów B and Sulejów C - Vistulian periglacial sands and muds. The conducted field studies allow for a correction to be made for sites Sulejów A and Sulejów C, where glacial till was found underneath the cover of sandy and silty periglacial forma- tions. The till, according to H. Brzeziński (1990), should be related to the Odranian Glaciation, whereas in the light of more recent interpretations by K. Turkowska (2006) - to the Wartanian Glaciation. The formations constitute the parent rock for sandy soils of different genetic types (AB), whose agricultural suitability was classified as poor rye complex (6) and very poor rye complex (7) (Województwo piotrkowskie. Mapa glebowo-rolnicza 1979). As regards soil valuation, the productivity of soils at plots Sulejów A and Sulejów C was qualified as class V, whereas at Sulejów B - as class VI of arable lands (http://geoportal.lodzkie.pl/ imap/). Photographic documentation and results of laboratory analyses from the plots of the Sulejów study area are presented in figures 5.13-5.17, and in tables 5.7-5.9.

STUDY PLOT SULEJÓW A (51ํ21'039N, 1954'847E, elevation 199 m a.s.l.)
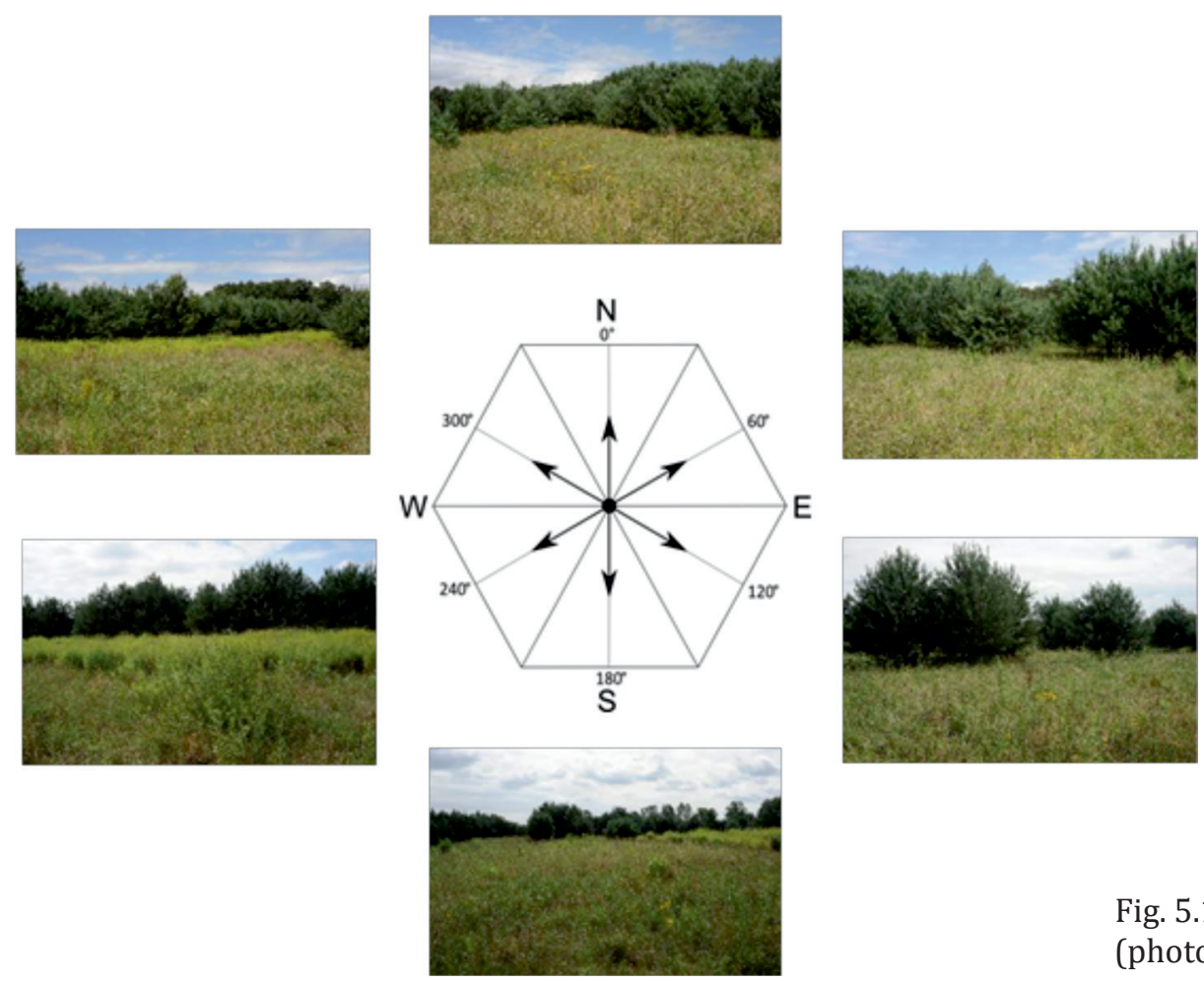

Fig. 5.13. Study plot Sulejów A (photo S. Krysiak, 2012) 


\section{Depth Profile description}

0-30 cm - humus, silty sand and compact silt, grey

30-60 cm - silty sand and silt with some fine sand, light beige

$60-90 \mathrm{~cm}$ - fine and medium sand, beige with rusty stains

90-110 cm - medium and coarse sand, rusty

110-140 cm - loamy sand turning into sandy loam, rusty brown

Table 5.7. Study plot Sulejów A. Granulometric and chemical properties of soil

\begin{tabular}{|c|c|c|c|c|c|c|c|c|c|c|c|c|}
\hline \multirow[b]{2}{*}{ Soil pit } & \multirow{2}{*}{$\begin{array}{c}\text { Sampling } \\
\text { depth } \\
\mathrm{cm}\end{array}$} & \multicolumn{9}{|c|}{$\begin{array}{c}\text { Grain size distribution } \\
\%\end{array}$} & \multirow{2}{*}{$\begin{array}{l}\text { Gra- } \\
\text { nulo- } \\
\text { metric } \\
\text { sub- } \\
\text { group }\end{array}$} & \multirow{2}{*}{$\begin{array}{c}\text { Specific } \\
\text { surface } \\
\text { area } \\
\mathrm{m}^{2} \cdot \mathrm{g}^{-1}\end{array}$} \\
\hline & & $\begin{array}{c}2.0- \\
1.0 \\
\mathrm{~mm}\end{array}$ & $\begin{array}{c}1.0- \\
0,5 \\
\mathrm{~mm}\end{array}$ & $\begin{array}{l}0.5- \\
0.25 \\
\mathrm{~mm}\end{array}$ & $\begin{array}{c}0.25- \\
0.1 \\
\mathrm{~mm}\end{array}$ & $\begin{array}{l}0.1- \\
0.05 \\
\mathrm{~mm}\end{array}$ & $\begin{array}{c}0.05- \\
0.02 \\
\mathrm{~mm}\end{array}$ & $\begin{array}{c}0.02- \\
0.005 \\
\mathrm{~mm}\end{array}$ & $\begin{array}{c}0.005- \\
0.002 \\
\mathrm{~mm}\end{array}$ & $\begin{array}{c}<0.002 \\
\mathrm{~mm}\end{array}$ & & \\
\hline Sulejów A & $0-20$ & 0.1 & 16.7 & 32.7 & 18.3 & 9.1 & 13.5 & 6.9 & 1.5 & 1.2 & pg & 0.1920 \\
\hline Sulejów A & $20-40$ & 0.1 & 17.8 & 35.7 & 19.4 & 6.6 & 10.0 & 6.4 & 2.2 & 1.7 & pg & 0.2230 \\
\hline Sulejów A & $90-110$ & 5.0 & 37.4 & 40.2 & 12.4 & 0.7 & 1.3 & 1.7 & 0.9 & 0.4 & $\mathrm{pl}$ & 0.0647 \\
\hline Sulejów A & $130-140$ & 0.4 & 15.3 & 25.9 & 21.8 & 7.3 & 6.0 & 10.6 & 7.3 & 5.3 & gp & 0.5730 \\
\hline
\end{tabular}

\begin{tabular}{|c|c|c|c|c|c|c|c|c|c|c|}
\hline \multirow[t]{2}{*}{ Soil pit } & \multirow{2}{*}{$\begin{array}{c}\text { Sampling } \\
\text { depth } \\
\text { cm }\end{array}$} & \multirow{2}{*}{$\begin{array}{c}\text { Humus } \\
\%\end{array}$} & \multirow{2}{*}{$\begin{array}{c}\mathrm{C} \text { total } \\
\%\end{array}$} & \multirow{2}{*}{$\begin{array}{c}\mathrm{N} \text { total } \\
\%\end{array}$} & \multirow[t]{2}{*}{$\mathrm{C} / \mathrm{N}$} & \multicolumn{2}{|c|}{$\begin{array}{l}\mathrm{pH} \\
\text { in }\end{array}$} & \multicolumn{3}{|c|}{$\begin{array}{c}\text { Available nutrients } \\
\mathrm{mg} / 100 \mathrm{~g}\end{array}$} \\
\hline & & & & & & $\mathrm{KCl}$ & $\mathrm{H}_{2} \mathrm{O}$ & $\mathrm{P}_{2} \mathrm{O}_{5}$ & $\mathrm{~K}_{2} \mathrm{O}$ & $\mathrm{Mg}$ \\
\hline Sulejów A & $0-20$ & 1.98 & 1.148 & 0.092 & 12.48 & 5.0 & 5.8 & 4.0 & 4.3 & 2.2 \\
\hline Sulejów A & $20-40$ & - & - & - & - & 4.3 & 6.3 & 2.4 & 1.1 & 1.3 \\
\hline Sulejów A & $90-110$ & - & - & - & - & 5.3 & 6.4 & 1.2 & 2.6 & 0.8 \\
\hline Sulejów A & $130-140$ & - & - & - & - & 5.2 & 6.6 & 2.9 & 2.9 & 5.9 \\
\hline
\end{tabular}

\begin{tabular}{|c|c|c|c|c|c|c|c|c|c|c|}
\hline \multirow[t]{2}{*}{ Soil pit } & \multirow{2}{*}{$\begin{array}{c}\text { Sampling } \\
\text { depth } \\
\text { cm }\end{array}$} & \multirow{2}{*}{$\begin{array}{l}\text { Hydrolytic } \\
\text { acidity } \\
\text { mmol/100g }\end{array}$} & \multicolumn{4}{|c|}{$\begin{array}{c}\text { Exchangeable cations } \\
\text { me } / 100 \mathrm{~g}\end{array}$} & \multicolumn{4}{|c|}{$\begin{array}{c}\text { Sorption capacity } \\
\mathrm{me} / 100 \mathrm{~g}\end{array}$} \\
\hline & & & $\mathrm{Ca}^{2+}$ & $\mathrm{Mg}^{2+}$ & $\mathrm{Na}^{+}$ & $\mathrm{K}^{+}$ & S & $\mathrm{T}$ & $(\mathrm{T}-\mathrm{S})$ & V, \% \\
\hline Sulejów A & $0-20$ & 2.86 & 2.30 & 0.187 & 0.052 & 0.115 & 2.654 & 5.514 & 2.86 & 48.1 \\
\hline Sulejów A & $20-40$ & 1.29 & 1.70 & 0.110 & 0.345 & 0.044 & 2.199 & 3.489 & 1.29 & 63.0 \\
\hline Sulejów A & $90-110$ & 0.55 & 0.80 & 0.065 & 0.017 & 0.062 & 0.944 & 1.494 & 0.55 & 63.2 \\
\hline Sulejów A & $130-140$ & 0.82 & 4.00 & 0.568 & 0.096 & 0.092 & 4.756 & 5.576 & 0.82 & 85.3 \\
\hline
\end{tabular}

Source: own elaboration.

\section{Characteristics of the flora and fungi - Sulejów A}

Approximately $80 \%$ of the study plot is covered with plants. A large number of plant species occur here (29). Three species dominate: Elymus repens (about 20\%), Senecio jacobea (10\%) and Solidago canadensis (Photo 6.1). The other species, which cover from 3-0.5\% include: Achillea millefolium, Agrimonia eupatoria, Anthriscus sylvestris, Antho- xanthum odoratum, Arrthenatherum elatior, Calamagrostis epigeios (Tab. 6.1). One tree species was also identified: Salix caprea.

Fungi of the plot consisted of only 3 species of macromycetes. The most frequent one was Inocybe asterospora (Tab. 7.1). 
STUDY PLOT SULEJóW B (51201'054N, 1954'886E, elevation 198 m a.s.l.)
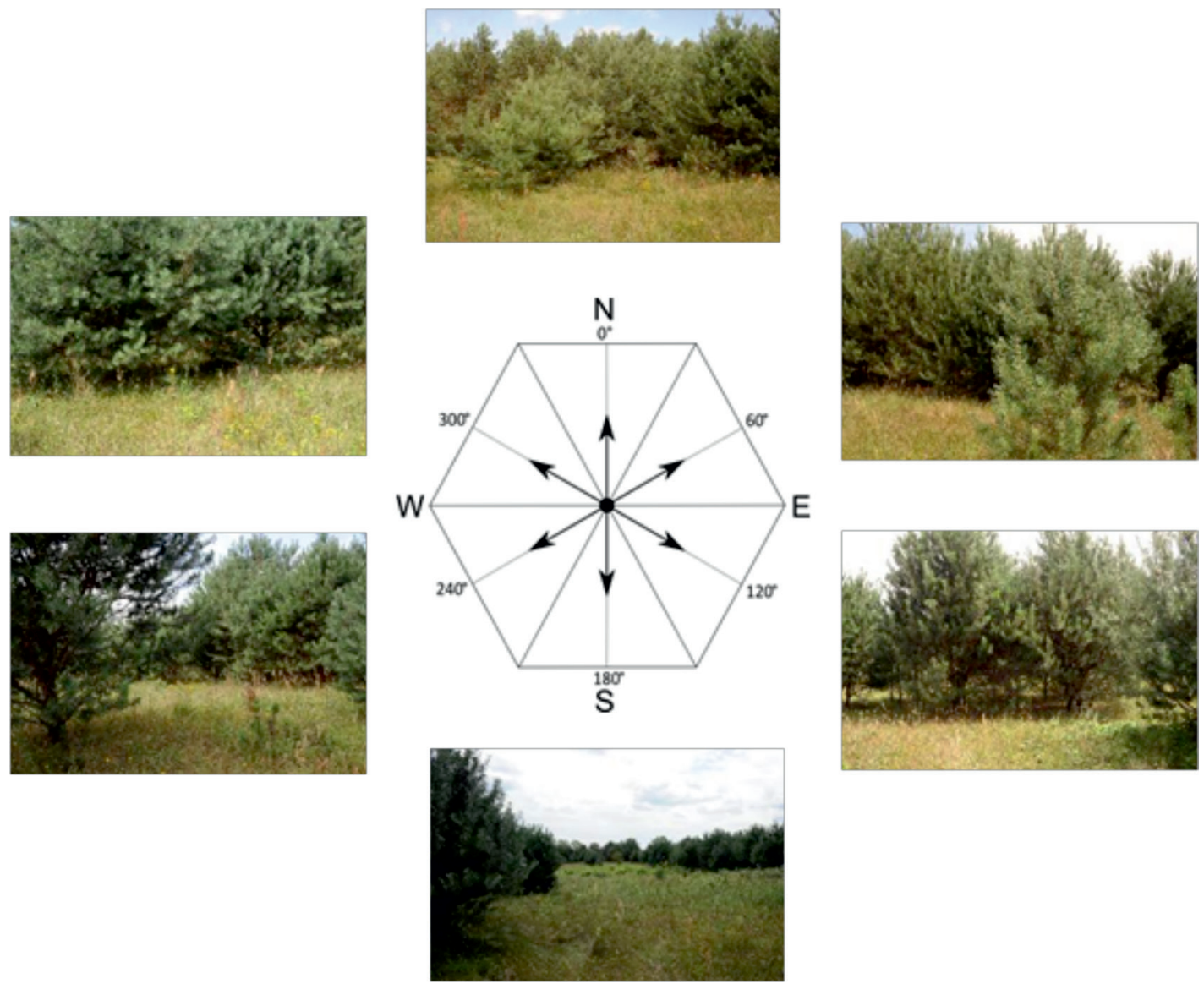

Fig. 5.14. Study plot Sulejów B (photo S. Krysiak, 2012)

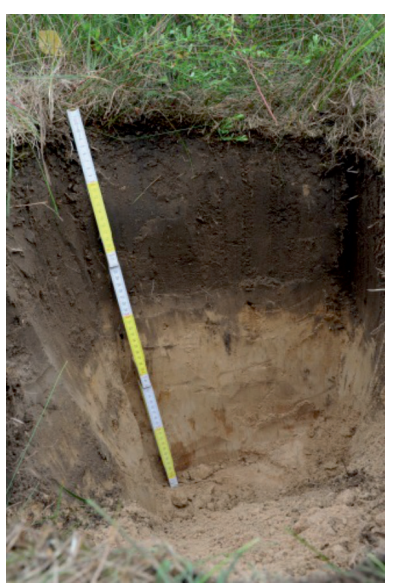

$$
\begin{aligned}
\text { Depth } & \text { Profile description } \\
0-30 \mathrm{~cm} \quad- & \text { humus, fine and silty sand with single cobbles } \\
& \text { of Scandinavian origin, dark brown and grey } \\
30-60 \mathrm{~cm}- & \text { fine and silty sand, beige } \\
60-90 \mathrm{~cm}- & \text { fine and medium sand, beige } \\
90-110 \mathrm{~cm}- & \text { medium and coarse sand with gravel of } 2 \text { to } 4 \mathrm{~mm} \\
& \text { in diameter, beige with rusty stains }
\end{aligned}
$$

Fig. 5.15. Soil pit in study plot Sulejów B (photo S. Krysiak, 2012) 
Table 5.8. Study plot Sulejów B. Granulometric and chemical properties of soil

\begin{tabular}{|c|c|c|c|c|c|c|c|c|c|c|c|c|}
\hline \multirow[b]{2}{*}{ Soil pit } & \multirow{2}{*}{$\begin{array}{c}\text { Sampling } \\
\text { depth } \\
\mathrm{cm}\end{array}$} & \multicolumn{9}{|c|}{$\begin{array}{c}\text { Grain size distribution } \\
\% \\
\end{array}$} & \multirow{2}{*}{$\begin{array}{l}\text { Gra- } \\
\text { nulo- } \\
\text { metric } \\
\text { sub- } \\
\text { group }\end{array}$} & \multirow{2}{*}{$\begin{array}{c}\text { Spe- } \\
\text { cific } \\
\text { surface } \\
\text { area } \\
\mathrm{m}^{2} \cdot \mathrm{g}^{-1}\end{array}$} \\
\hline & & $\begin{array}{c}2.0- \\
1.0 \\
\mathrm{~mm}\end{array}$ & $\begin{array}{c}1.0- \\
0,5 \\
\mathrm{~mm}\end{array}$ & $\begin{array}{l}0.5- \\
0.25 \\
\mathrm{~mm}\end{array}$ & $\begin{array}{c}0.25- \\
0.1 \\
\mathrm{~mm}\end{array}$ & $\begin{array}{l}0.1- \\
0.05 \\
\mathrm{~mm}\end{array}$ & $\begin{array}{c}0.05- \\
0.02 \\
\mathrm{~mm}\end{array}$ & $\begin{array}{c}0.02- \\
0.005 \\
\mathrm{~mm}\end{array}$ & $\begin{array}{c}0.005- \\
0.002 \\
\mathrm{~mm}\end{array}$ & $\begin{array}{c}<0.002 \\
\mathrm{~mm}\end{array}$ & & \\
\hline Sulejów B & $0-20$ & 0.1 & 16.7 & 36.7 & 21.3 & 7.1 & 9.9 & 5.5 & 1.5 & 1.1 & ps & 0.1690 \\
\hline Sulejów B & $20-40$ & 0.0 & 13.0 & 34.1 & 20.3 & 7.6 & 12.4 & 7.8 & 2.6 & 2.2 & $\mathrm{pg}$ & 0.2760 \\
\hline Sulejów B & $90-110$ & 0.8 & 32.1 & 46.7 & 16.2 & 0.7 & 1.1 & 1.1 & 0.9 & 0.5 & $\mathrm{pl}$ & 0.0629 \\
\hline
\end{tabular}

\begin{tabular}{|c|c|c|c|c|c|c|c|c|c|c|}
\hline \multirow[t]{2}{*}{ Soil pit } & \multirow{2}{*}{$\begin{array}{c}\text { Sampling } \\
\text { depth } \\
\text { cm }\end{array}$} & \multirow{2}{*}{$\begin{array}{c}\text { Humus } \\
\%\end{array}$} & \multirow{2}{*}{$\begin{array}{l}\text { C total } \\
\%\end{array}$} & \multirow{2}{*}{$\begin{array}{l}\mathrm{N} \text { total } \\
\%\end{array}$} & \multirow[t]{2}{*}{$\mathrm{C} / \mathrm{N}$} & \multicolumn{2}{|c|}{$\begin{array}{l}\mathrm{pH} \\
\text { in }\end{array}$} & \multicolumn{3}{|c|}{$\begin{array}{l}\text { Available nutrients } \\
\mathrm{mg} / 100 \mathrm{~g}\end{array}$} \\
\hline & & & & & & $\mathrm{KCl}$ & $\mathrm{H}_{2} \mathrm{O}$ & $\mathrm{P}_{2} \mathrm{O}_{5}$ & $\mathrm{~K}_{2} \mathrm{O}$ & $\mathrm{Mg}$ \\
\hline Sulejów B & $0-20$ & 1.49 & 0.864 & 0.073 & 11.84 & 4.4 & 5.4 & 7.6 & 5.8 & 1.2 \\
\hline Sulejów B & $20-40$ & - & - & - & - & 4.9 & 6.1 & 1.2 & 4.2 & 0.8 \\
\hline Sulejów B & $90-110$ & - & - & - & - & 5.2 & 6.4 & 1.0 & 2.4 & 0.8 \\
\hline
\end{tabular}

\begin{tabular}{|c|c|c|c|c|c|c|c|c|c|c|}
\hline \multirow[t]{2}{*}{ Soil pit } & \multirow{2}{*}{$\begin{array}{l}\text { Sampling } \\
\text { depth } \\
\text { cm }\end{array}$} & \multirow{2}{*}{$\begin{array}{l}\text { Hydrolytic } \\
\text { acidity } \\
\text { mmol/100g }\end{array}$} & \multicolumn{4}{|c|}{$\begin{array}{l}\text { Exchangeable cations } \\
\text { me } / 100 \mathrm{~g}\end{array}$} & \multicolumn{4}{|c|}{$\begin{array}{c}\text { Sorption capacity } \\
\text { me } / 100 \mathrm{~g}\end{array}$} \\
\hline & & & $\mathrm{Ca}^{2+}$ & $\mathrm{Mg}^{2+}$ & $\mathrm{Na}^{+}$ & $\mathrm{K}^{+}$ & S & $\mathrm{T}$ & $(\mathrm{T}-\mathrm{S})$ & $\mathrm{V}, \%$ \\
\hline Sulejów B & $0-20$ & 7.25 & 1.10 & 0.107 & 0.026 & 0.159 & 1.392 & 8.642 & 7.25 & 16.1 \\
\hline Sulejów B & $20-40$ & 1.12 & 0.80 & 0.065 & 0.017 & 0.120 & 1.002 & 2.122 & 1.12 & 47.2 \\
\hline Sulejów B & $90-110$ & 0.42 & 0.60 & 0.057 & 0.017 & 0.061 & 0.735 & 1.155 & 0.42 & 63.6 \\
\hline
\end{tabular}

Source: own elaboration.

\section{Characteristics of the flora and fungi - Sulejów B}

Approximately $50 \%$ of the study plot is covered with plants. A small number of plant species occur here (9). Three species dominate: Rumex acetosa (about 20\%), Stachys arvensis (10\%) and Rubus sp. Other species, covering from 1-0.5\%, include: Melandrium album, Artemisia campestris, Chelichrysum arenaria (Tab. 6.1).

Fungi of the plot are sparse. Only 6 species of macromycetes were found here, of which the most abundant were Bovista plumbea and Marasmius oreades (Tab. 7.1). 
STUDY PLOT SULEJÓW C (51²21'015N, 1954'933E, elevation 193 m a.s.l.)
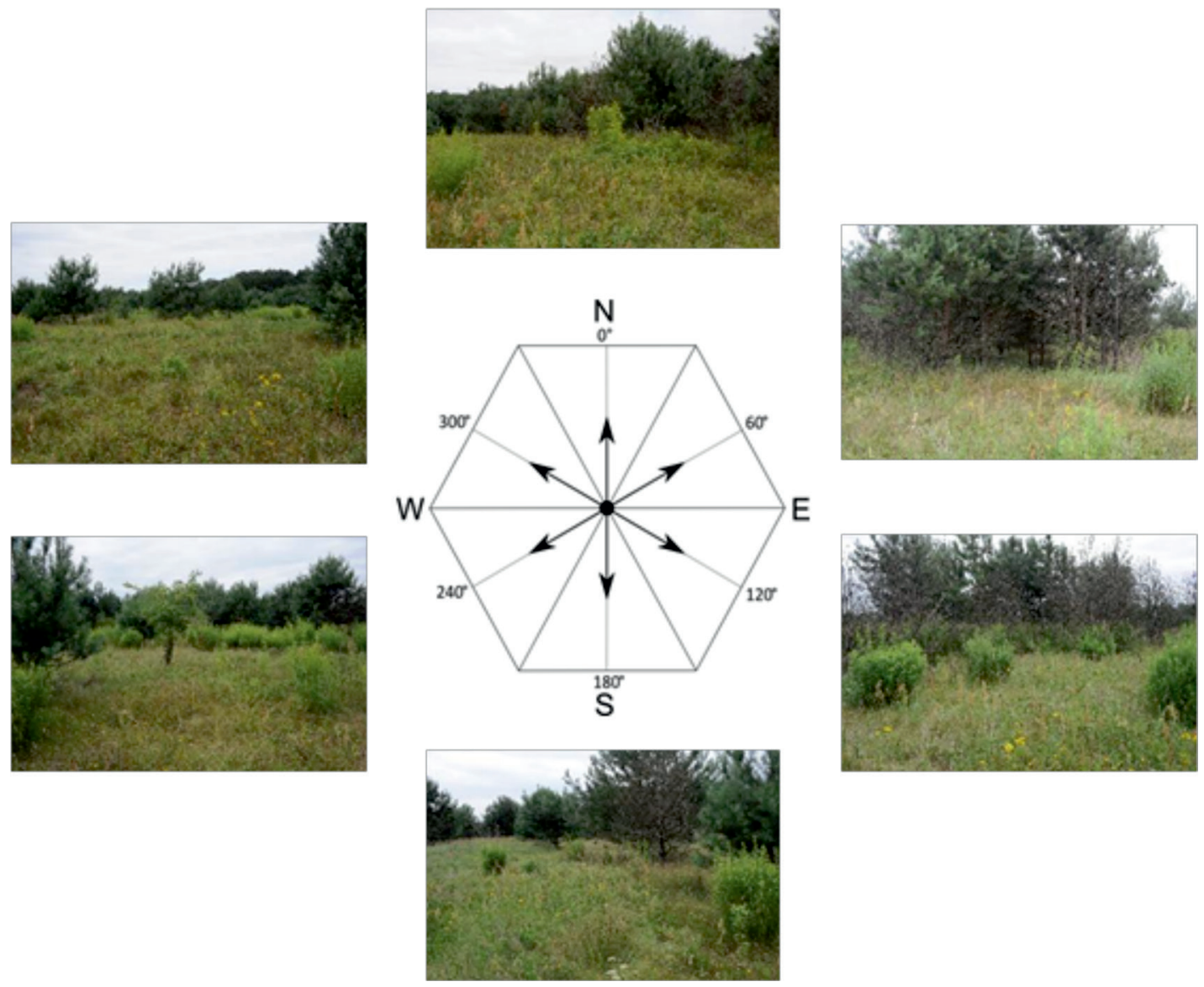

Fig. 5.16. Study plot Sulejów C (photo S. Krysiak, 2012)

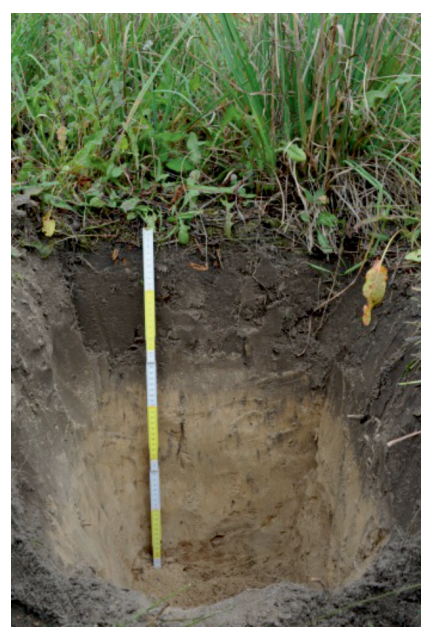

$$
\begin{aligned}
\text { Depth } & \text { Profile description } \\
0-25 \mathrm{~cm}- & \text { humus, fine and silty sand with single cobbles of } \\
& \begin{array}{l}
\text { Scandinavian origin, diameter of up to } 10 \mathrm{~cm} \text {, dark } \\
\text { brown }
\end{array} \\
25-60 \mathrm{~cm}- & \text { fine and silty sand, beige with dark grey stains } \\
60-70 \mathrm{~cm}- & \text { medium sand with cobbles of Scandinavian origin, } \\
& \text { diameter from } 4 \text { to } 10 \mathrm{~cm} \text { (stone pavement horizon) } \\
70-80 \mathrm{~cm}- & \text { loamy sand, brown and rusty } \\
80-110 \mathrm{~cm}- & \text { sandy loam, light brown }
\end{aligned}
$$

Fig. 5.17. Soil pit in study plot Sulejów C (photo S. Krysiak, 2012) 
Table 5.9. Study plot Sulejów C. Granulometric and chemical properties of soil

\begin{tabular}{|c|c|c|c|c|c|c|c|c|c|c|c|c|}
\hline \multirow[b]{2}{*}{ Soil pit } & \multirow{2}{*}{$\begin{array}{c}\text { Sampling } \\
\text { depth } \\
\text { cm }\end{array}$} & \multicolumn{9}{|c|}{$\begin{array}{c}\text { Grain size distribution } \\
\%\end{array}$} & \multirow{2}{*}{$\begin{array}{l}\text { Gra- } \\
\text { nulo- } \\
\text { metric } \\
\text { sub- } \\
\text { group }\end{array}$} & \multirow{2}{*}{$\begin{array}{c}\text { Specific } \\
\text { surface } \\
\text { area } \\
\mathrm{m}^{2} \cdot \mathrm{g}^{-1}\end{array}$} \\
\hline & & $\begin{array}{c}2.0- \\
1.0 \\
\mathrm{~mm}\end{array}$ & $\begin{array}{c}1.0- \\
0,5 \\
\mathrm{~mm}\end{array}$ & $\begin{array}{l}0.5- \\
0.25 \\
\mathrm{~mm}\end{array}$ & $\begin{array}{c}0.25- \\
0.1 \\
\mathrm{~mm}\end{array}$ & $\begin{array}{l}0.1- \\
0.05 \\
\mathrm{~mm}\end{array}$ & $\begin{array}{c}0.05- \\
0.02 \\
\mathrm{~mm}\end{array}$ & $\begin{array}{c}0.02- \\
0.005 \\
\mathrm{~mm}\end{array}$ & $\begin{array}{c}0.005- \\
0.002 \\
\mathrm{~mm}\end{array}$ & $\begin{array}{c}<0.002 \\
\mathrm{~mm}\end{array}$ & & \\
\hline Sulejów C & $0-20$ & 0.0 & 14.3 & 30.5 & 18.0 & 10.1 & 14.5 & 8.7 & 2.2 & 1.8 & $\mathrm{pg}$ & 0.254 \\
\hline Sulejów C & $20-40$ & 0.4 & 16.3 & 31.1 & 18.3 & 8.7 & 13.1 & 7.7 & 2.4 & 2.0 & $\mathrm{pg}$ & 0.266 \\
\hline Sulejów C & $90-110$ & 1.0 & 17.0 & 25.4 & 20.6 & 7.0 & 5.6 & 10.2 & 7.4 & 5.8 & gp & 0.608 \\
\hline
\end{tabular}

\begin{tabular}{|c|c|c|c|c|c|c|c|c|c|c|}
\hline \multirow[t]{2}{*}{ Soil pit } & \multirow{2}{*}{$\begin{array}{l}\text { Sampling } \\
\text { depth } \\
\mathrm{cm}\end{array}$} & \multirow{2}{*}{$\begin{array}{c}\text { Humus } \\
\%\end{array}$} & \multirow{2}{*}{$\begin{array}{c}\text { C total } \\
\%\end{array}$} & \multirow{2}{*}{$\begin{array}{l}\mathrm{N} \text { total } \\
\%\end{array}$} & \multirow[t]{2}{*}{$\mathrm{C} / \mathrm{N}$} & \multicolumn{2}{|c|}{$\begin{array}{l}\mathrm{pH} \\
\text { in }\end{array}$} & \multicolumn{3}{|c|}{$\begin{array}{c}\text { Available nutrients } \\
\mathrm{mg} / 100 \mathrm{~g}\end{array}$} \\
\hline & & & & & & $\mathrm{KCl}$ & $\mathrm{H}_{2} \mathrm{O}$ & $\mathrm{P}_{2} \mathrm{O}_{5}$ & $\mathrm{~K}_{2} \mathrm{O}$ & $\mathrm{Mg}$ \\
\hline Sulejów C & $0-20$ & 1.94 & 1.125 & 0.094 & 11.97 & 4.6 & 5.5 & 3.9 & 5.5 & 2.7 \\
\hline Sulejów C & $20-40$ & - & - & - & - & 5.1 & 6.1 & 2.4 & 1.1 & 2.2 \\
\hline Sulejów C & $90-110$ & - & - & - & - & 4.9 & 6.3 & 2.2 & 3.4 & 8.9 \\
\hline
\end{tabular}

\begin{tabular}{|c|c|c|c|c|c|c|c|c|c|c|}
\hline \multirow{2}{*}{ Soil pit } & \multirow{2}{*}{$\begin{array}{c}\text { Sampling } \\
\text { depth } \\
\text { cm }\end{array}$} & \multirow{2}{*}{$\begin{array}{l}\text { Hydrolytic } \\
\text { acidity } \\
\text { mmol/100g }\end{array}$} & \multicolumn{4}{|c|}{$\begin{array}{c}\text { Exchangeable cations } \\
\mathrm{me} / 100 \mathrm{~g}\end{array}$} & \multicolumn{4}{|c|}{$\begin{array}{c}\text { Sorption capacity } \\
\text { me/100 g }\end{array}$} \\
\hline & & & $\mathrm{Ca}^{2+}$ & $\mathrm{Mg}^{2+}$ & $\mathrm{Na}^{+}$ & $\mathrm{K}^{+}$ & S & $\mathrm{T}$ & $(\mathrm{T}-\mathrm{S})$ & $\mathrm{V}, \%$ \\
\hline Sulejów C & $0-20$ & 3.56 & 2.10 & 0.248 & 0.043 & 0.159 & 2.550 & 6.110 & 3.56 & 41.7 \\
\hline Sulejów C & $20-40$ & 1.58 & 1.30 & 0.205 & 0.026 & 0.049 & 1.580 & 3.160 & 1.58 & 50.0 \\
\hline Sulejów C & $90-110$ & 0.98 & 4.10 & 0.840 & 0.087 & 0.126 & 5.153 & 6.133 & 0.98 & 84.0 \\
\hline
\end{tabular}

Source: own elaboration.

\section{Characteristics of the flora and fungi - Sulejów C}

Approximately $80 \%$ of the study plot is covered with plants; rich in plant species (30). Among plants, the largest areas are covered with grasses. Four species dominate: Poa pratensis (about $20 \%$ ), Rumex acetosa (about 15\%), Dactylis glomerata (about 10\%) and Agrostis capillaris (about $7 \%$ ). The share of other species does not exceed $1-0.5 \%$. They include: Achillea millefolium,
Anthriscus sylvestris, Chelichrysum arenaria, Vicia hirsuta (Tab. 6.1). The area also features a few saplings of Pyrus communis.

Fungi of the plot are represented by 9 species of macromycetes. The most frequent one is Crinipellis scabella, occurring on grasses, mainly on Agrostis capillaris, Arrhenia lobata and Vascellum pratense (Tab. 7.1).

\section{Analysis of granulometric composition and chemical properties of soils in the Sulejów study plot group}

At all the plots down to the depth of $40 \mathrm{~cm}$, the content of silty and clayey fractions allowed the analysed sediments to be qualified as the granulometric subgroups of loamy sand (pg) and slightly loamy sand (ps). It is presumed that it is a Vistulian periglacial cover, underlined at plot Sulejów $\mathrm{C}$ with a clear stone pavement horizon, found at the depth of $60-70 \mathrm{~cm}$. Additionally, in the case of plots Sulejów A and C, the water balance of the habitats is influenced by sandy loam. At plot Sulejów
A, it occurs at the depth of $90 \mathrm{~cm}$, at plot Sulejów $\mathrm{C}-$ at $130 \mathrm{~cm}$.

As regards agronomic classification, the granulometric composition of the humus layer at the analysed sites qualify them to soils of category I and II (very light and light soils).

The $\mathrm{pH}$ reaction of most analysed series was acidic. At plots Sulejów A and Sulejów B, some individual series showed a very acidic reaction ( $\mathrm{pH}$ in $\mathrm{KCl}<4.5$ ). 
At the analysed plots, the content of available phosphorus $\left(\mathrm{P}_{2} \mathrm{O}_{5}\right)$, potassium $\left(\mathrm{K}_{2} \mathrm{O}\right)$ and magnesium $(\mathrm{Mg})$ oscillated at a level corresponding to very low and low class of availability (class $\mathrm{V}$ and IV). Only at plot Sulejów C did the content of available magnesium in the series from the depth of 0-20 cm correspond to class III, whereas in loams at the depth of $90-110 \mathrm{~cm}$ - to class II. In the analysed series, the degree of saturation of the sorptive complex with alkaline cations $\mathrm{Ca}^{2+}, \mathrm{Mg}^{2+}$, $\mathrm{Na}^{+}$and $\mathrm{K}^{+}$was fairly high, ranging from $41.7 \%$ to $85.3 \%$. Only in the sample from the depth of $0-20 \mathrm{~cm}$ at plot Sulejów B it was significantly lower $-16.1 \%$.

Plots Sulejów A and Sulejów C were characterised by a considerably larger number of plant species (29 and 30) than Sulejów B, where the number was 9 .

\section{PISKORZENIEC STUDY PLOT GROUP}

The Piskorzeniec study plot group is located about $1 \mathrm{~km}$ from the Przedbórz Landscape Park, near the eastern border of the Łódź Voivodeship, in Przedbórz Commune. The surface sediments at all three plots (Piskorzeniec A, Piskorzeniec B and Piskorzeniec C) are cover aeolian sands, deposited upon postglacial sands and gravels from the Warta Glaciation (Jurkiewicz 1962). The deposits constitute the parent rock for sandy soils of different genetic types (AB), whose agricultural suitability was classified as very poor rye complex (7) and poor and very poor grasslands (3z) (Wo- jewództwo piotrkowskie. Mapa glebowo-rolnicza 1979). As regards soil valuation, the productivity of these soils was qualified as class VI of arable lands and class VI of grasslands (http://geoportal.lodzkie.pl/imap/). The sites of the Piskorzeniec study area represent young abandoned lands, several years old, not taken over by trees and shrubs yet. In their vicinity, there are some older abandoned lands with a large share of pine trees. Photographic documentation and results of laboratory analyses from the Piskorzeniec study plot group are presented in figures 5.18-5.21 and in tables 5.10-5.12.

\section{STUDY PLOT PISKORZENIEC A (51 $01^{\circ}$ '896N, 2001'720E, elevation 239 m a.s.l.)}
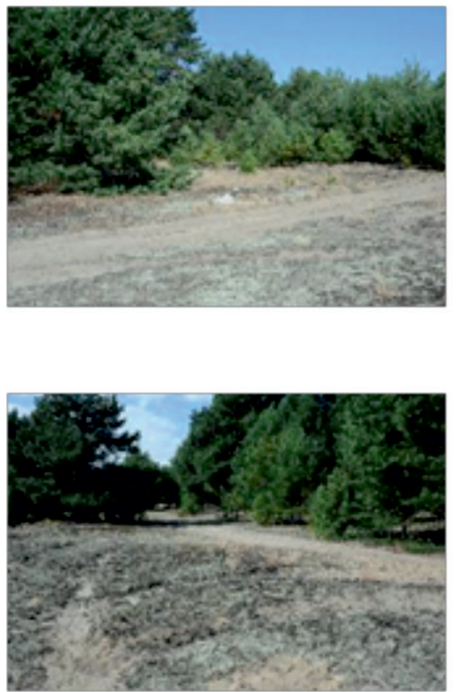
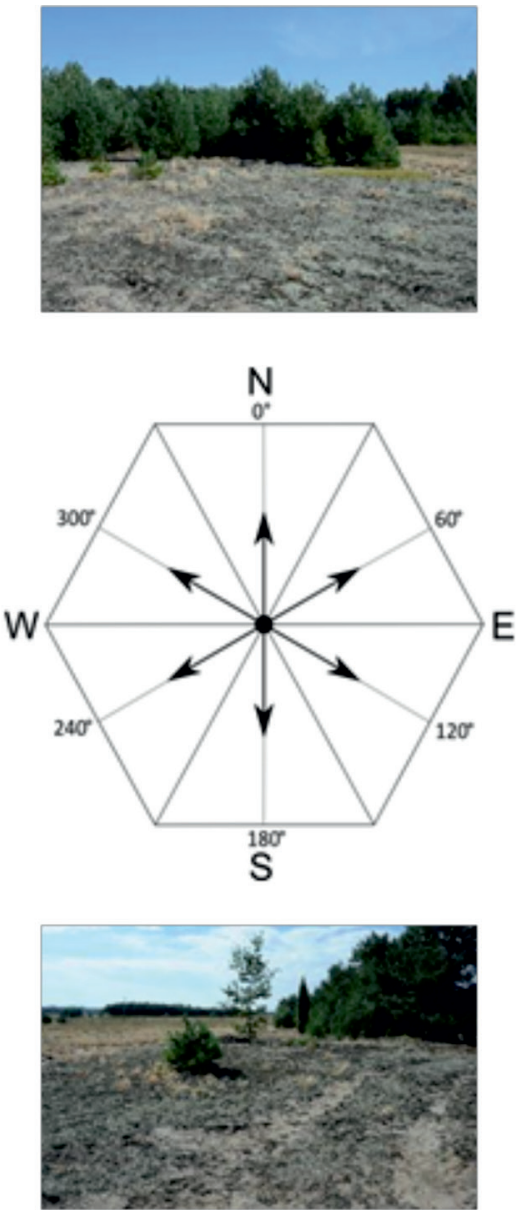
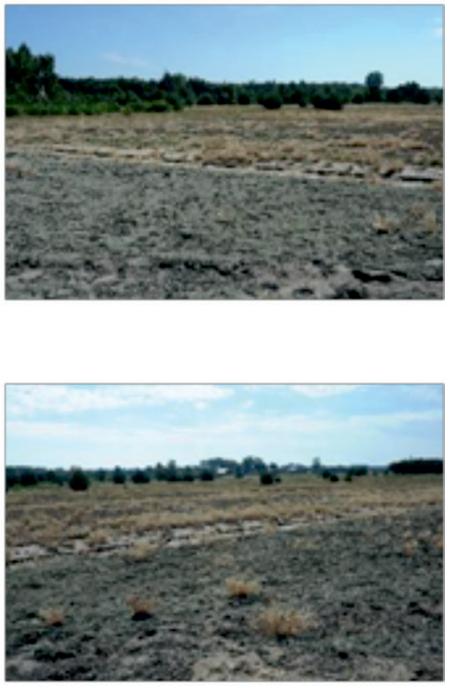

Fig. 5.18. Study plot Piskorzeniec A (photo S. Krysiak, 2012) 


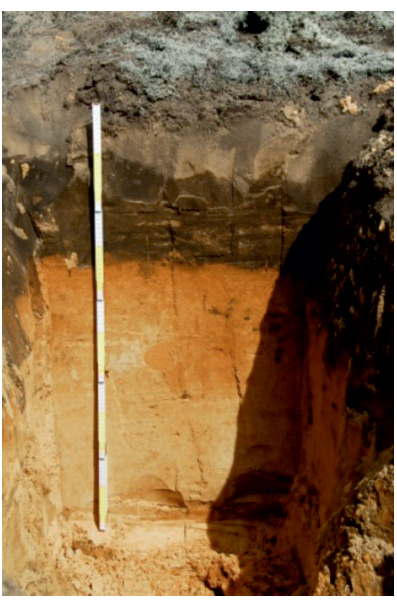

Depth Profile description

0-28 cm - humus, fine and medium sand, grey

$28-40 \mathrm{~cm}$ - fine and medium sand, rusty yellow

40-90 cm - fine and medium sand, yellow

$90-110 \mathrm{~cm}$ - fine and medium sand, beige

Fig. 5.19. Soil pit in study plot Sulejów C (photo S. Krysiak, 2012)

Table 5.10. Study plot Piskorzeniec A. Granulometric and chemical properties of soil

\begin{tabular}{|c|c|c|c|c|c|c|c|c|c|c|c|c|}
\hline \multirow[b]{2}{*}{ Soil pit } & \multirow{2}{*}{$\begin{array}{c}\text { Sampling } \\
\text { depth } \\
\mathrm{cm}\end{array}$} & \multicolumn{9}{|c|}{$\begin{array}{c}\text { Grain size distribution } \\
\%\end{array}$} & \multirow{2}{*}{$\begin{array}{l}\text { Gra- } \\
\text { nulo- } \\
\text { metric } \\
\text { sub- } \\
\text { group }\end{array}$} & \multirow{2}{*}{$\begin{array}{c}\text { Specific } \\
\text { surface } \\
\text { area } \\
\mathrm{m}^{2} \cdot \mathrm{g}^{-1}\end{array}$} \\
\hline & & $\begin{array}{c}2.0- \\
1.0 \\
\mathrm{~mm}\end{array}$ & $\begin{array}{c}1.0- \\
0.5 \\
\mathrm{~mm}\end{array}$ & $\begin{array}{c}0.5- \\
0.25 \\
\mathrm{~mm} \\
\end{array}$ & $\begin{array}{c}0.25- \\
0.1 \\
\mathrm{~mm}\end{array}$ & $\begin{array}{l}0.1- \\
0.05 \\
\mathrm{~mm}\end{array}$ & $\begin{array}{c}0.05- \\
0.02 \\
\mathrm{~mm}\end{array}$ & $\begin{array}{c}0.02- \\
0.005 \\
\mathrm{~mm}\end{array}$ & $\begin{array}{c}0.005- \\
0.002 \\
\mathrm{~mm}\end{array}$ & $\begin{array}{c}<0.002 \\
\mathrm{~mm}\end{array}$ & & \\
\hline $\begin{array}{l}\text { Piskorze- } \\
\text { niec A }\end{array}$ & $0-20$ & 0.4 & 27.4 & 47.2 & 19.8 & 0.9 & 1.5 & 1.8 & 0.8 & 0.2 & $\mathrm{pl}$ & 0.0542 \\
\hline $\begin{array}{l}\text { Piskorze- } \\
\text { niec A }\end{array}$ & $20-40$ & 0.3 & 28.9 & 46.9 & 16.9 & 1.7 & 2.1 & 1.9 & 0.9 & 0.4 & $\mathrm{pl}$ & 0.0694 \\
\hline $\begin{array}{l}\text { Piskorze- } \\
\text { niec A }\end{array}$ & $90-110$ & 0.0 & 20.9 & 49.0 & 25.4 & 1.6 & 1.9 & 0.9 & 0.3 & 0.0 & $\mathrm{pl}$ & 0.0337 \\
\hline
\end{tabular}

\begin{tabular}{|c|c|c|c|c|c|c|c|c|c|c|}
\hline Soil pit & $\begin{array}{c}\text { Sampling } \\
\text { depth } \\
\mathrm{cm}\end{array}$ & $\begin{array}{c}\text { Humus } \\
\%\end{array}$ & $\begin{array}{c}\mathrm{C} \text { total } \\
\%\end{array}$ & $\begin{array}{c}\mathrm{N} \text { total } \\
\%\end{array}$ & \multicolumn{2}{|c|}{$\mathrm{C} / \mathrm{N}$} & \multicolumn{3}{|c|}{$\mathrm{pH}$} & \multicolumn{3}{|c|}{$\begin{array}{c}\text { Available nutrients } \\
\mathrm{mg} / 100 \mathrm{~g}\end{array}$} \\
\cline { 6 - 11 } & & & & $\mathrm{KCl}$ & $\mathrm{H}_{2} \mathrm{O}$ & $\mathrm{P}_{2} \mathrm{O}_{5}$ & $\mathrm{~K}_{2} \mathrm{O}$ & $\mathrm{Mg}$ \\
\hline $\begin{array}{c}\text { Piskorze- } \\
\text { niec A }\end{array}$ & $0-20$ & 0.64 & 0.371 & 0.020 & 18.55 & 4.2 & 4.9 & 5.0 & 0.5 & 0.3 \\
\hline $\begin{array}{c}\text { Piskorze- } \\
\text { niec A }\end{array}$ & $20-40$ & - & - & - & - & 4.5 & 4.8 & 2.6 & 0.5 & 0.3 \\
\hline $\begin{array}{c}\text { Piskorze- } \\
\text { niec A }\end{array}$ & $90-110$ & - & - & - & - & 4.6 & 5.0 & 1.9 & 0.4 & 0.2 \\
\hline
\end{tabular}

\begin{tabular}{|c|c|c|c|c|c|c|c|c|c|c|}
\hline & \multirow{2}{*}{ Sampling } & \multirow{2}{*}{$\begin{array}{c}\text { Hydrolytic } \\
\text { acidity } \\
\text { depth } \\
\mathrm{cm}\end{array}$} & \multicolumn{4}{|c|}{$\begin{array}{c}\text { Exchangeable cations } \\
\mathrm{mm} / 100 \mathrm{~g}\end{array}$} & \multicolumn{4}{c|}{$\begin{array}{c}\text { Sorption capacity } \\
\mathrm{me} / 100 \mathrm{~g}\end{array}$} \\
\cline { 5 - 12 } & & $\mathrm{Ca}^{2+}$ & $\mathrm{Mg}^{2+}$ & $\mathrm{Na}^{+}$ & $\mathrm{K}^{+}$ & $\mathrm{S}$ & $\mathrm{T}$ & $(\mathrm{T}-\mathrm{S})$ & $\mathrm{V}, \%$ \\
\hline $\begin{array}{c}\text { Piskorze- } \\
\text { niec A }\end{array}$ & $0-20$ & 2.13 & 0.10 & 0.017 & 0.017 & 0.013 & 0.147 & 2.277 & 2.13 & 6.5 \\
\hline $\begin{array}{c}\text { Piskorze- } \\
\text { niec A }\end{array}$ & $20-40$ & 1.68 & 0.05 & 0.017 & 0.009 & 0.013 & 0.089 & 1.769 & 1.68 & 5.0 \\
\hline $\begin{array}{c}\text { Piskorze- } \\
\text { niec A }\end{array}$ & $90-110$ & 1.07 & $\mathrm{ND}$ & 0.017 & 0.017 & 0.005 & 0.039 & 1.109 & 1.07 & 3.5 \\
\hline
\end{tabular}

Source: own elaboration. 


\section{Characteristics of the flora and fungi - Piskorzeniec A}

Approximately $7 \%$ of the study plot is covered with plants. Very poor in species of vascular plants (2). The plot is dominated by lichens, of which the most abundant is Cladonia arbuscula, which covers about $50 \%$. It is accompanied by Cladonia rangiferina and Cladonia coccifera, which cover about $20 \%$ of the area. Among the lichens, the- re are small tufts of Corynephorus canescens and a few specimens of Arnoseris minima (Tab. 6.1).

Fungi of the plot are very sparse. They are represented by only 2 species of macromycetes: $B o$ vista plumbea and Lycoperdon nigrescens. Several isolated sporocarps of these species occurred in the area (Tab. 7.1).

STUDY PLOT PISKORZENIEC B (51 $03^{\circ} 881 \mathrm{~N}, 20^{\circ} 01^{\prime} 729 \mathrm{E}$, elevation $239 \mathrm{~m}$ a.s.l.)
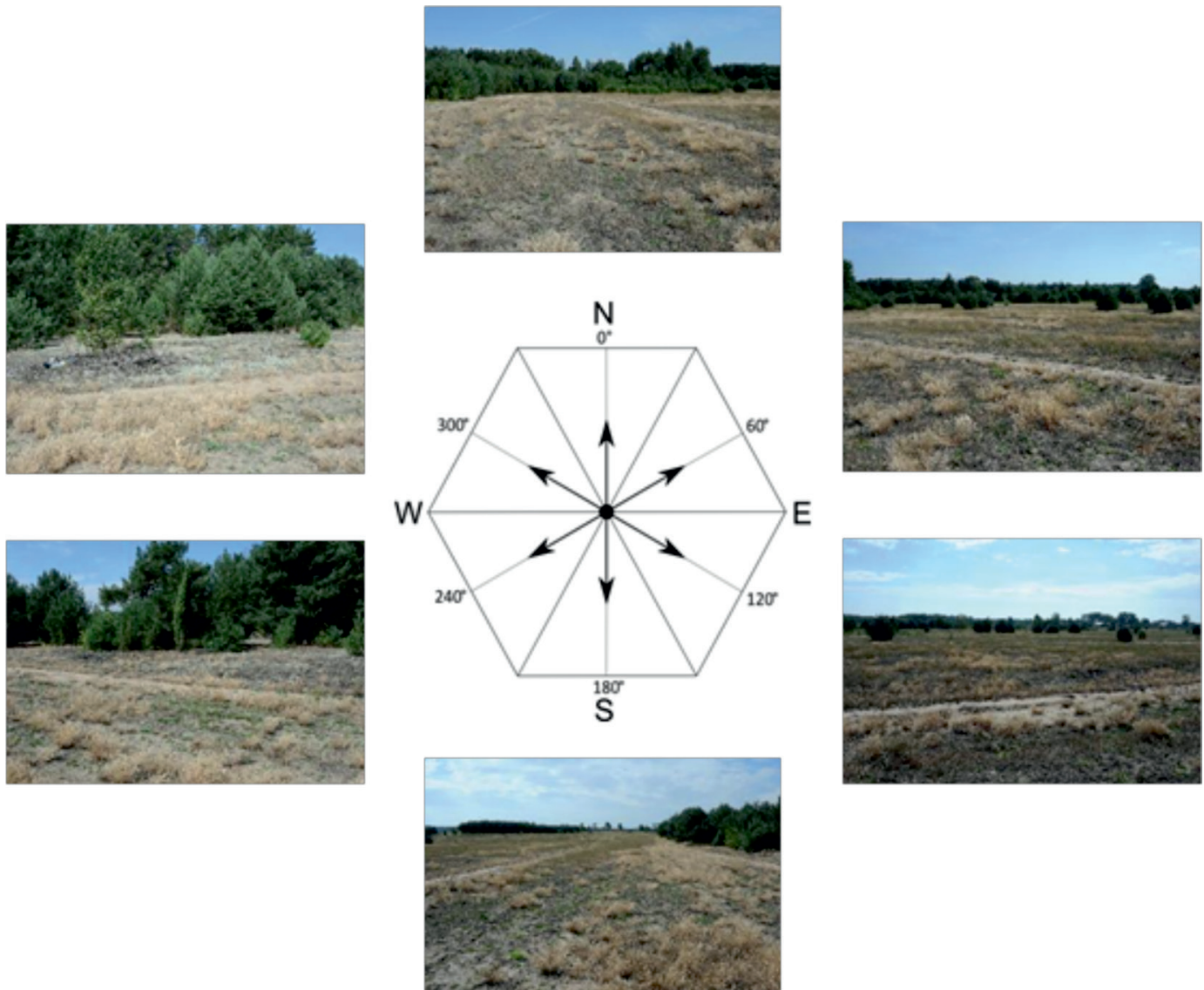

Fig. 5.20. Study plot Piskorzeniec B (photo S. Krysiak, 2012)

Depth Profile description

0-28 cm - humus, fine and medium sand, grey

$28-40 \mathrm{~cm}$ - fine and medium sand, rusty yellow

40-90 cm - fine and medium sand, yellow

90-110 cm - fine and medium sand, beige 
Table 5.11. Study plot Piskorzeniec B. Granulometric and chemical properties of soil

\begin{tabular}{|c|c|c|c|c|c|c|c|c|c|c|c|c|}
\hline \multirow[b]{2}{*}{ Soil pit } & \multirow{2}{*}{$\begin{array}{l}\text { Sampling } \\
\text { depth } \\
\mathrm{cm}\end{array}$} & \multicolumn{9}{|c|}{$\begin{array}{c}\text { Grain size distribution } \\
\%\end{array}$} & \multirow{2}{*}{$\begin{array}{l}\text { Gra- } \\
\text { nulo- } \\
\text { metric } \\
\text { sub- } \\
\text { group }\end{array}$} & \multirow{2}{*}{$\begin{array}{c}\text { Specific } \\
\text { surface } \\
\text { area } \\
\mathrm{m}^{2} \cdot \mathrm{g}^{-1}\end{array}$} \\
\hline & & $\begin{array}{c}2.0- \\
1.0 \\
\mathrm{~mm}\end{array}$ & $\begin{array}{c}1.0- \\
0.5 \\
\mathrm{~mm}\end{array}$ & $\begin{array}{l}0.5- \\
0.25 \\
\mathrm{~mm}\end{array}$ & $\begin{array}{c}0.25- \\
0.1 \\
\mathrm{~mm}\end{array}$ & $\begin{array}{l}0.1- \\
0.05 \\
\mathrm{~mm}\end{array}$ & $\begin{array}{c}0.05- \\
0.02 \\
\mathrm{~mm}\end{array}$ & $\begin{array}{c}0.02- \\
0.005 \\
\mathrm{~mm}\end{array}$ & $\begin{array}{c}0.005- \\
0.002 \\
\mathrm{~mm}\end{array}$ & $\begin{array}{c}<0.002 \\
\mathrm{~mm}\end{array}$ & & \\
\hline $\begin{array}{l}\text { Piskorze- } \\
\text { niec B }\end{array}$ & $0-20$ & 0.1 & 22.9 & 45.9 & 22.7 & 2.3 & 3.2 & 2.2 & 0.7 & 0.0 & $\mathrm{pl}$ & 0.0541 \\
\hline $\begin{array}{l}\text { Piskorze- } \\
\text { niec B }\end{array}$ & $20-40$ & 0.0 & 19.9 & 45.9 & 26.1 & 2.2 & 2.5 & 2.0 & 0.9 & 0.4 & $\mathrm{pl}$ & 0.0747 \\
\hline $\begin{array}{l}\text { Piskorze- } \\
\text { niec B }\end{array}$ & $90-110$ & 0.0 & 18.8 & 52.0 & 26.7 & 0.8 & 1.7 & 0.0 & 0.0 & 0.0 & $\mathrm{pl}$ & 0.0233 \\
\hline
\end{tabular}

\begin{tabular}{|c|c|c|c|c|c|c|c|c|c|c|}
\hline Soil pit & $\begin{array}{c}\text { Sampling } \\
\text { depth } \\
\mathrm{cm}\end{array}$ & $\begin{array}{c}\text { Humus } \\
\%\end{array}$ & $\begin{array}{c}\mathrm{C} \text { total } \\
\%\end{array}$ & $\begin{array}{c}\mathrm{N} \text { total } \\
\%\end{array}$ & $\mathrm{C} / \mathrm{N}$ & \multicolumn{3}{|c|}{$\begin{array}{c}\mathrm{pH} \\
\mathrm{in}\end{array}$} & \multicolumn{3}{|c|}{$\begin{array}{c}\text { Available nutrients } \\
\mathrm{mg} / 100 \mathrm{~g}\end{array}$} \\
\cline { 6 - 11 } & & & $\mathrm{KCl}$ & $\mathrm{H}_{2} \mathrm{O}$ & $\mathrm{P}_{2} \mathrm{O}_{5}$ & $\mathrm{~K}_{2} \mathrm{O}$ & $\mathrm{Mg}$ \\
\hline $\begin{array}{c}\text { Piskorze- } \\
\text { niec B }\end{array}$ & $0-20$ & 1.26 & 0.731 & 0.048 & 15.22 & 3.9 & 4.4 & 12.6 & 0.3 & 0.2 \\
\hline $\begin{array}{c}\text { Piskorze- } \\
\text { niec B }\end{array}$ & $20-40$ & - & - & - & - & 4.4 & 4.7 & 2.6 & 0.4 & 0.3 \\
\hline $\begin{array}{c}\text { Piskorze- } \\
\text { niec B }\end{array}$ & $90-110$ & - & - & - & - & 4.6 & 5.0 & 2.0 & 0.6 & 0.2 \\
\hline
\end{tabular}

\begin{tabular}{|c|c|c|c|c|c|c|c|c|c|c|}
\hline & \multirow{2}{*}{$\begin{array}{c}\text { Sampling } \\
\text { Septh } \\
\mathrm{cm}\end{array}$} & $\begin{array}{c}\text { Hydrolytic } \\
\text { acidity } \\
\mathrm{mmol} / 100 \mathrm{~g}\end{array}$ & \multicolumn{4}{|c|}{$\begin{array}{c}\text { Exchangeable cations } \\
\mathrm{me} / 100 \mathrm{~g}\end{array}$} & \multicolumn{4}{c|}{$\begin{array}{c}\text { Sorption capacity } \\
\mathrm{me} / 100 \mathrm{~g}\end{array}$} \\
\cline { 5 - 13 } & & & $\mathrm{Ca}^{2+}$ & $\mathrm{Mg}^{2+}$ & $\mathrm{Na}^{+}$ & $\mathrm{K}^{+}$ & $\mathrm{S}$ & $\mathrm{T}$ & $(\mathrm{T}-\mathrm{S})$ & $\mathrm{V}, \%$ \\
\hline $\begin{array}{c}\text { Piskorze- } \\
\text { niec B }\end{array}$ & $0-20$ & 3.49 & 0.10 & 0.017 & 0.017 & 0.013 & 0.147 & 3.637 & 3.49 & 4.0 \\
\hline $\begin{array}{c}\text { Piskorze- } \\
\text { niec B }\end{array}$ & $20-40$ & 1.69 & $\mathrm{ND}$ & 0.017 & 0.017 & 0.013 & 0.047 & 1.737 & 1.69 & 2.7 \\
\hline $\begin{array}{c}\text { Piskorze- } \\
\text { niec B }\end{array}$ & $90-110$ & 0.99 & $\mathrm{ND}$ & 0.017 & 0.017 & 0.013 & 0.047 & 1.037 & 0.99 & 4.5 \\
\hline
\end{tabular}

Source: own elaboration.

\section{Characteristics of the flora and fungi - Piskorzeniec B}

Approximately $10 \%$ of the study plot is covered with plants. Very poor in species of vascular plants (3). The identified species are: Hieracium pilosella, Anthoxanthum aristatum and Spergularia rubra. Area coverage with specimens of any of the species did not exceed 3\% (Tab. 6.1).
Fungi of the plot are exceptionally sparse. They are represented by only 1 species of macromycetes: Bovista plumbea. Two sporocarps of this species were found in the area (Tab. 7.1). 


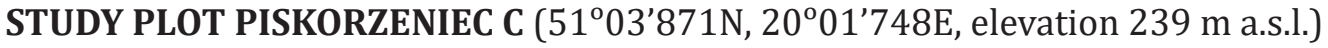
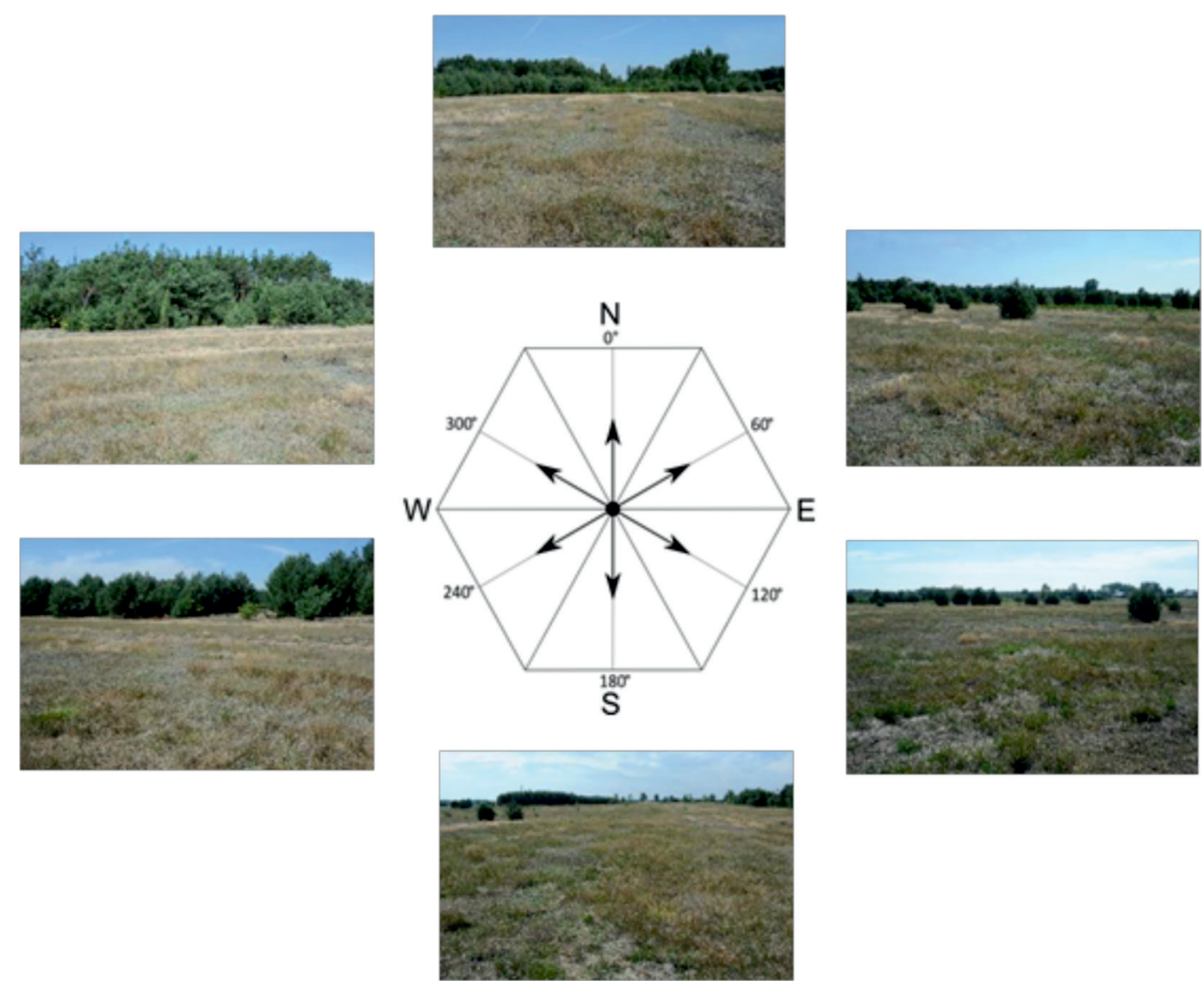

Fig. 5.21. Study plot Piskorzeniec C (photo S. Krysiak, 2012)

$\begin{aligned} \text { Depth } & \text { Profile description } \\ 0-20 \mathrm{~cm} & - \text { humus, fine and medium sand, light grey } \\ 20-40 \mathrm{~cm} & - \text { fine and medium sand, yellow } \\ 40-70 \mathrm{~cm} & - \text { fine and medium sand, light yellow } \\ 70-110 \mathrm{~cm} & \text { - fine and medium sand, rusty with grey stains }\end{aligned}$

Table 5.12. Study plot Piskorzeniec C. Granulometric and chemical properties of soil

\begin{tabular}{|c|c|c|c|c|c|c|c|c|c|c|c|c|}
\hline \multirow[b]{2}{*}{ Soil pit } & \multirow{2}{*}{$\begin{array}{c}\text { Sampling } \\
\text { depth } \\
\mathrm{cm}\end{array}$} & \multicolumn{9}{|c|}{$\begin{array}{c}\text { Grain size distribution } \\
\% \\
\end{array}$} & \multirow{2}{*}{$\begin{array}{l}\text { Gra- } \\
\text { nulo- } \\
\text { metric } \\
\text { sub- } \\
\text { group }\end{array}$} & \multirow{2}{*}{$\begin{array}{c}\text { Specific } \\
\text { surface } \\
\text { area } \\
\mathrm{m}^{2} \cdot \mathrm{g}^{-1}\end{array}$} \\
\hline & & $\begin{array}{c}2.0- \\
1.0 \\
\mathrm{~mm}\end{array}$ & $\begin{array}{c}1.0- \\
0.5 \\
\mathrm{~mm}\end{array}$ & $\begin{array}{l}0.5- \\
0.25 \\
\mathrm{~mm}\end{array}$ & $\begin{array}{c}0.25- \\
0.1 \\
\mathrm{~mm}\end{array}$ & $\begin{array}{l}0.1- \\
0.05 \\
\mathrm{~mm}\end{array}$ & $\begin{array}{c}0.05- \\
0.02 \\
\mathrm{~mm}\end{array}$ & $\begin{array}{c}0.02- \\
0.005 \\
\mathrm{~mm}\end{array}$ & $\begin{array}{c}0.005- \\
0.002 \\
\mathrm{~mm}\end{array}$ & $\begin{array}{c}<0.002 \\
\mathrm{~mm}\end{array}$ & & \\
\hline $\begin{array}{l}\text { Piskorze- } \\
\text { niec C }\end{array}$ & $0-20$ & 0,0 & 20,1 & 43,8 & 23,3 & 4,4 & 5,1 & 2,6 & 0,7 & 0,0 & $\mathrm{pl}$ & 0,0600 \\
\hline $\begin{array}{l}\text { Piskorze- } \\
\text { niec C }\end{array}$ & $20-40$ & 0,0 & 20,4 & 47,9 & 26,4 & 1,4 & 1,7 & 1,4 & 0,7 & 0,2 & $\mathrm{pl}$ & 0,0519 \\
\hline $\begin{array}{c}\text { Piskorze- } \\
\text { niec C }\end{array}$ & $90-110$ & 0,0 & 21,4 & 50,0 & 28,2 & 0,4 & 0,0 & 0,0 & 0,0 & 0,0 & $\mathrm{pl}$ & 0,0204 \\
\hline
\end{tabular}


Table 5.12. Study plot Piskorzeniec C. Granulometric and chemical properties of soil (continued)

\begin{tabular}{|c|c|c|c|c|c|c|c|c|c|c|}
\hline Soil pit & $\begin{array}{c}\text { Sampling } \\
\text { depth } \\
\mathrm{cm}\end{array}$ & $\begin{array}{c}\text { Humus } \\
\%\end{array}$ & $\begin{array}{c}\mathrm{C} \text { total } \\
\%\end{array}$ & $\begin{array}{c}\mathrm{N} \text { total } \\
\%\end{array}$ & $\mathrm{C} / \mathrm{N}$ & \multicolumn{3}{|c|}{$\begin{array}{c}\mathrm{pH} \\
\text { in }\end{array}$} & & \multicolumn{3}{|c|}{$\begin{array}{c}\text { Available nutrients } \\
\mathrm{mg} / 100 \mathrm{~g}\end{array}$} \\
\cline { 6 - 11 } \\
$\begin{array}{c}\text { Piskorze- } \\
\text { niec C }\end{array}$ & $0-20$ & 2.18 & 1.265 & 0.085 & 14.88 & 3.9 & 4.3 & 15.6 & 0.9 & 0.3 \\
\hline $\begin{array}{c}\text { Piskorze- } \\
\text { niec C }\end{array}$ & $20-40$ & - & - & - & - & 4.4 & 4.7 & 2.3 & 0.5 & 0.2 \\
\hline $\begin{array}{c}\text { Piskorze- } \\
\text { niec C }\end{array}$ & $90-110$ & - & - & - & - & 4.6 & 5.3 & 1.5 & 0.4 & 0.2 \\
\hline
\end{tabular}

\begin{tabular}{|c|c|c|c|c|c|c|c|c|c|c|}
\hline & \multirow{2}{*}{$\begin{array}{c}\text { Sampling } \\
\text { depth } \\
\mathrm{cm}\end{array}$} & \multirow{2}{*}{$\begin{array}{c}\text { Hydrolytic } \\
\text { acidity } \\
\mathrm{mmol} / 100 \mathrm{~g}\end{array}$} & \multicolumn{4}{|c|}{$\begin{array}{c}\text { Exchangeable cations } \\
\mathrm{me} / 100 \mathrm{~g}\end{array}$} & \multicolumn{4}{c|}{$\begin{array}{c}\text { Sorption capacity } \\
\text { me } / 100 \mathrm{~g}\end{array}$} \\
\cline { 6 - 13 } & & $\mathrm{Ca}^{2+}$ & $\mathrm{Mg}^{2+}$ & $\mathrm{Na}^{+}$ & $\mathrm{K}^{+}$ & $\mathrm{S}$ & $\mathrm{T}$ & $(\mathrm{T}-\mathrm{S})$ & $\mathrm{V}, \%$ \\
\hline $\begin{array}{c}\text { Piskorze- } \\
\text { niec C }\end{array}$ & $0-20$ & 5.14 & 0.10 & 0.017 & 0.009 & 0.038 & 0.164 & 5.304 & 5.14 & 3.1 \\
\hline $\begin{array}{c}\text { Piskorze- } \\
\text { niec C }\end{array}$ & $20-40$ & 1.76 & $\mathrm{ND}$ & 0.017 & 0.009 & 0.018 & 0.044 & 1.804 & 1.76 & 2.4 \\
\hline $\begin{array}{c}\text { Piskorze- } \\
\text { niec C }\end{array}$ & $90-110$ & 0.90 & $\mathrm{ND}$ & 0.017 & 0.009 & 0.013 & 0.039 & 0.939 & 0.90 & 4.2 \\
\hline
\end{tabular}

Source: own elaboration.

\section{Characteristics of the flora and fungi - Piskorzeniec C}

Approximately $70 \%$ of the study plot is covered with plants. Poor in species of vascular plants (9). The dominating species are: Hieracium pilosella (about 40\%) and Poa compressa (about 20\%). Coverage with specimens of other species did not exceed 3\%. They included: Agrostis capillaris,
Apera spica-venti, Arnoseris minima, Carex ovalis, and Pinus sylvestris (Tab. 6.1).

Fungi of the plot are sparse. They are represented with only three species of macromycetes: Amanita muscaria, Bovista plumbea and Suillus luteus (Tab. 7.1).

\section{Analysis of granulometric composition and chemical properties of soils in the Piskorzeniec study plot group}

The Piskorzeniec study plot group represents very poor, dry habitats related to the occurrence of cover aeolian sands. The percentage share of granulometric fractions indicates a good sorting of the sediments. At all three analysed plots: $\mathrm{Pi}$ skorzeniec $\mathrm{A}, \mathrm{B}$ and $\mathrm{C}$, the dominating role is played by the sandy fraction, mainly the subfraction of medium sand, whose share in the analysed horizons ranges from 43 to $50 \%$. Very coarse sands (1 to $2 \mathrm{~mm}$ ) and the clayey fraction represent an insignificant share (below 0.4\%). All the analysed samples were qualified as the granulometric subgroup of loose sands ( $\mathrm{pl}$ ) with low values of specific surface area, ranging from 0.0233 to $0.0747 \mathrm{~m}^{2} \cdot \mathrm{g}^{-1}$.

As regards agronomic classification, the granulometric composition of the analysed sediments qualify them as soils of category I (very light soils).
At all analysed plots - Piskorzeniec A, B and C, samples from the depths of $0-20 \mathrm{~cm}$ and $20-40 \mathrm{~cm}$ revealed very acidic reaction, whereas below, at the depth of $90-110 \mathrm{~cm}$, the reaction was acidic. At plots $B$ and $C$, in the sample from the $0-10 \mathrm{~cm}$ horizon, medium and high content of available phosphorus $\left(\mathrm{P}_{2} \mathrm{O}_{5}\right)$ was recorded, whereas in deeper horizons and along the entire profile at plot $\mathrm{A}$, the content was low and very low. In all horizons at sites Piskorzeniec A, B and C, a very low content of available potassium $\left(\mathrm{K}_{2} \mathrm{O}\right)$ and magnesium $(\mathrm{Mg}$ ) was found. An insignificant share of alkaline cations $\mathrm{Ca}^{2+}, \mathrm{Mg}^{2+}, \mathrm{Na}^{+}$and $\mathrm{K}^{+}$, expressed by very low degree of saturation of the sorptive complex $(\mathrm{V})$, ranging from 2.7 to $6.5 \%$, confirms the low agricultural suitability of the area. 


\section{WOLA ŻYCIŃSKA STUDY PLOT GROUP}

The Wola Życińska study plot group is located $6 \mathrm{~km}$ away from the borders of the Przedbórz Landscape Park, in Wielgomłyny Commune. The surface sediments at plots Wola Życińska A and Wola Życińska C include sands, sands with gravels and boulders, glacial and partially fluvioglacial, not separated, originated during the period of the Odranian Glaciation, whereas at plot Wola Życińska B - Odranian glacial tills (Szajn 1978). The deposits constitute the parent rock for sandy soils of various genetic types $(\mathrm{AB})$, whose agricultural suitability was classified as poor rye complex (6). As regards soil valuation, productivity of soils at plot Wola Życińska A was classified as class IVb, at plots Wola Życińska B and C - as class V of arable lands (http://geoportal.lodzkie.pl/imap/). Photographic documentation and results of laboratory analyses from the plots of the Wola Życińska study area are presented in figures 5.22-5.24 and in tables 5.13-5.15.

STUDY PLOT WOLA ŻYCIŃSKA A (5057'174N, 19048'677E, elevation 215 m a.s.l.)
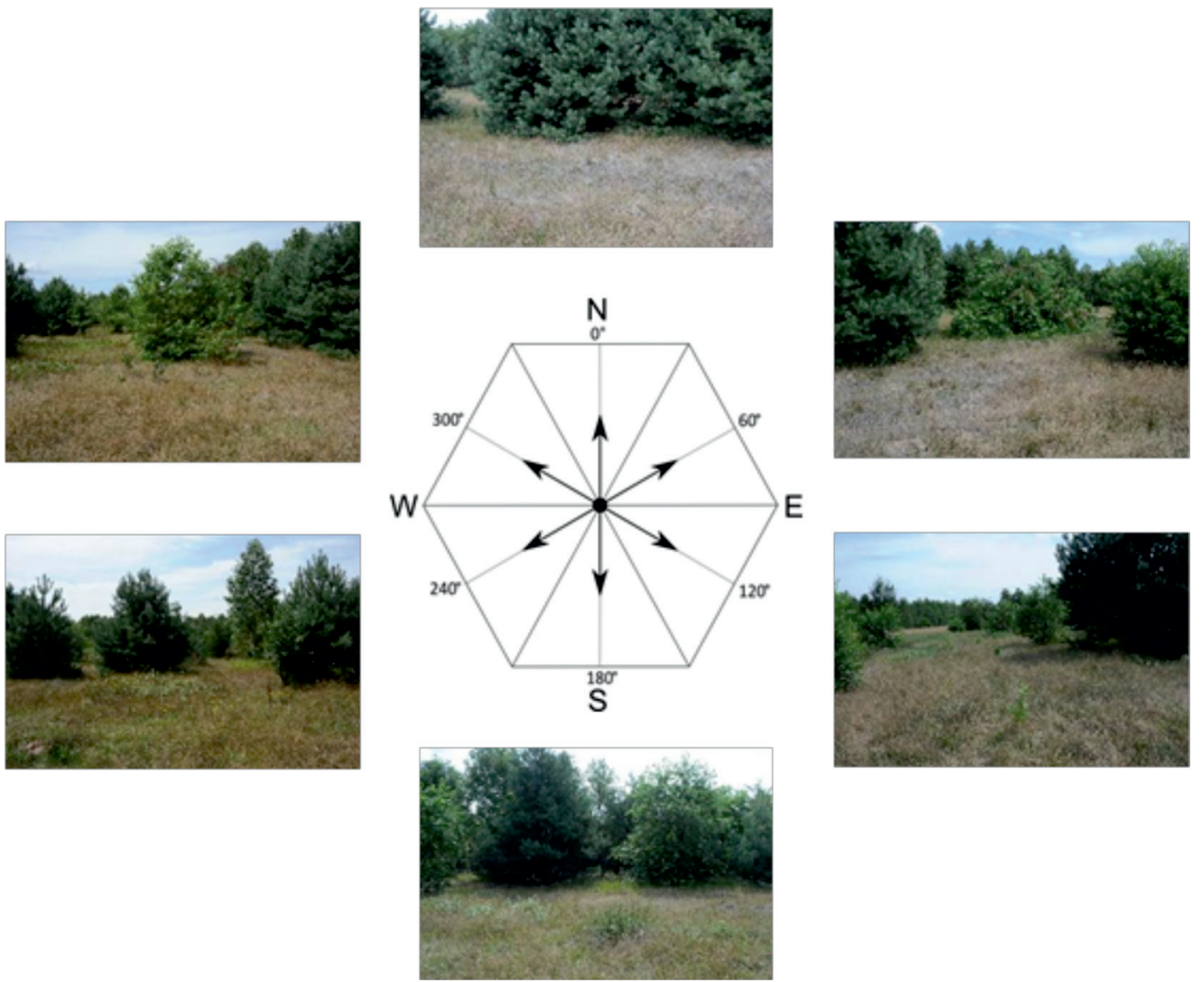

Fig. 5.22. Study plot Wola Życińska A (photo S. Krysiak, 2012)

Depth Profile description

0-30 cm - humus, fine sand and silty sand with single cobbles of Scandinavian origin, light grey

$30-50 \mathrm{~cm}$ - unsorted sand with some silty sand and single granules of gravel, grey and rusty

$50-60 \mathrm{~cm}$ - unsorted sands with cobbles of Scandinavian and local origin with 6 to $7 \mathrm{~cm}$ in diameter, beige

$60-90 \mathrm{~cm}$ - unsorted sands with single pebbles, yellow

90-120 cm - unsorted sands, rusty

120-165 cm - coarse and medium sands with single pebbles, rusty 
Table 5.13. Study plot Wola Życińska A. Granulometric and chemical properties of soil

\begin{tabular}{|c|c|c|c|c|c|c|c|c|c|c|c|c|}
\hline \multirow[b]{2}{*}{ Soil pit } & \multirow{2}{*}{$\begin{array}{c}\text { Sampling } \\
\text { depth } \\
\text { cm }\end{array}$} & \multicolumn{9}{|c|}{$\begin{array}{c}\text { Grain size distribution } \\
\%\end{array}$} & \multirow{2}{*}{$\begin{array}{l}\text { Gra- } \\
\text { nulo- } \\
\text { metric } \\
\text { sub- } \\
\text { group }\end{array}$} & \multirow{2}{*}{$\begin{array}{c}\text { Specific } \\
\text { surface } \\
\text { area } \\
\mathrm{m}^{2} \cdot \mathrm{g}^{-1}\end{array}$} \\
\hline & & $\begin{array}{c}2.0- \\
1.0 \\
\mathrm{~mm}\end{array}$ & $\begin{array}{l}1.0- \\
0.5 \\
\mathrm{~mm}\end{array}$ & $\begin{array}{l}0.5- \\
0.25 \\
\mathrm{~mm}\end{array}$ & $\begin{array}{c}0.25- \\
0.1 \\
\mathrm{~mm}\end{array}$ & $\begin{array}{l}0.1- \\
0.05 \\
\mathrm{~mm}\end{array}$ & $\begin{array}{c}0.05- \\
0.02 \\
\mathrm{~mm}\end{array}$ & $\begin{array}{c}0.02- \\
0.005 \\
\mathrm{~mm}\end{array}$ & $\begin{array}{c}0.005- \\
0.002 \\
\mathrm{~mm}\end{array}$ & $\begin{array}{c}<0.002 \\
\mathrm{~mm}\end{array}$ & & \\
\hline $\begin{array}{l}\text { W. Życiń- } \\
\text { ska A }\end{array}$ & $0-20$ & 0.5 & 16.7 & 29.3 & 23.7 & 8.4 & 10.2 & 7.6 & 2.1 & 1.5 & pg & 0.2150 \\
\hline $\begin{array}{l}\text { W. Życiń- } \\
\text { ska A }\end{array}$ & $20-40$ & 1.7 & 20.8 & 33.3 & 24.0 & 5.8 & 6.3 & 5.2 & 1.7 & 1.2 & ps & 0.1670 \\
\hline $\begin{array}{l}\text { W. Życiń- } \\
\text { ska A }\end{array}$ & $90-110$ & 5.1 & 38.7 & 37.5 & 13.9 & 2.2 & 0.4 & 1.2 & 0.8 & 0.2 & $\mathrm{pl}$ & 0.0473 \\
\hline $\begin{array}{l}\text { W. Życiń- } \\
\text { ska A }\end{array}$ & $160-165$ & 3.5 & 47.3 & 44.4 & 4.8 & 0.0 & 0.0 & 0.0 & 0.0 & 0.0 & $\mathrm{pl}$ & 0.0130 \\
\hline
\end{tabular}

\begin{tabular}{|c|c|c|c|c|c|c|c|c|c|c|}
\hline Soil pit & $\begin{array}{c}\text { Sampling } \\
\text { depth } \\
\mathrm{cm}\end{array}$ & $\begin{array}{c}\text { Humus } \\
\%\end{array}$ & $\begin{array}{c}\text { C total } \\
\%\end{array}$ & $\begin{array}{c}\text { N total } \\
\%\end{array}$ & $\mathrm{C} / \mathrm{N}$ & \multicolumn{3}{|c|}{$\begin{array}{c}\mathrm{pH} \\
\mathrm{in}\end{array}$} & \multicolumn{3}{|c|}{$\begin{array}{c}\text { Available nutrients } \\
\mathrm{mg} / 100 \mathrm{~g}\end{array}$} \\
\cline { 6 - 11 } & & & $\mathrm{KCl}$ & $\mathrm{H}_{2} \mathrm{O}$ & $\mathrm{P}_{2} \mathrm{O}_{5}$ & $\mathrm{~K}_{2} \mathrm{O}$ & $\mathrm{Mg}$ \\
\hline $\begin{array}{c}\text { W. Życiń- } \\
\text { ska A }\end{array}$ & $0-20$ & 1.85 & 1.073 & 0.082 & 13.09 & 3.8 & 4.5 & 15.0 & 1.3 & 0.5 \\
\hline $\begin{array}{c}\text { W. Życiń- } \\
\text { ska A }\end{array}$ & $20-40$ & - & - & - & - & 4.4 & 4.9 & 1.9 & 0.4 & 0.3 \\
\hline $\begin{array}{c}\text { W. Życiń- } \\
\text { ska A }\end{array}$ & $90-110$ & - & - & - & - & 4.5 & 5.8 & 1.2 & 4.3 & 1.6 \\
\hline $\begin{array}{c}\text { W. Życiń- } \\
\text { ska A }\end{array}$ & $160-165$ & - & - & - & - & 4.6 & 6.0 & 1.3 & 3.3 & 1.2 \\
\hline
\end{tabular}

\begin{tabular}{|c|c|c|c|c|c|c|c|c|c|c|}
\hline & \multirow{2}{*}{$\begin{array}{c}\text { Sampling } \\
\text { depth } \\
\mathrm{cm}\end{array}$} & \multirow{2}{*}{$\begin{array}{c}\text { Hydrolytic } \\
\text { acidity } \\
\mathrm{mmol} / 100 \mathrm{~g}\end{array}$} & \multicolumn{4}{|c|}{$\begin{array}{c}\text { Exchangeable cations } \\
\mathrm{me} / 100 \mathrm{~g}\end{array}$} & \multicolumn{4}{c|}{$\begin{array}{c}\text { Sorption capacity } \\
\mathrm{me} / 100 \mathrm{~g}\end{array}$} \\
\cline { 5 - 12 } & & $\mathrm{Ca}^{2+}$ & $\mathrm{Mg}^{+}$ & $\mathrm{Na}^{+}$ & $\mathrm{K}^{+}$ & $\mathrm{S}$ & $\mathrm{T}$ & $(\mathrm{T}-\mathrm{S})$ & $\mathrm{V}, \%$ \\
\hline $\begin{array}{c}\text { W. Życiń- } \\
\text { ska A }\end{array}$ & $0-20$ & 5.48 & 0.20 & 0.033 & 0.035 & 0.050 & 0.318 & 5.798 & 5.48 & 5.5 \\
\hline $\begin{array}{c}\text { W. Życiń- } \\
\text { ska A }\end{array}$ & $20-40$ & 2.21 & 0.15 & 0.017 & 0.017 & 0.013 & 0.197 & 2.407 & 2.21 & 8.2 \\
\hline $\begin{array}{c}\text { W. Życiń- } \\
\text { ska A }\end{array}$ & $90-110$ & 0.73 & 0.70 & 0.150 & 0.026 & 0.087 & 0.963 & 1.693 & 0.73 & 57.0 \\
\hline $\begin{array}{c}\text { W. Życiń- } \\
\text { ska A }\end{array}$ & $160-165$ & 0.43 & 0.50 & 0.117 & 0.026 & 0.062 & 0.705 & 1.135 & 0.43 & 62.1 \\
\hline
\end{tabular}

Source: own elaboration.

\section{Characteristics of the flora and fungi - Wola Życińska A}

Approximately $80 \%$ of the study plot is covered with plants. Among plants, three species dominate: Agrostis capillaris (about 30\%), Holcus mollis (30\%) and Hieracium pilosella (about 10\%). The share of other species does not exceed $2-0.5 \%$ of area coverage (Tab. 6.1). The plot also features saplings of trees and shrubs: Pyrus communis and Padus serotina (Photo 6.2).
Fungi of the plot are represented by 11 species of macromycetes. The most frequently found species is Crinipellis scabella, which occurs on grasses, mainly on Agrostis capillaris. Among land fungi, the most abundant species was Marasmius oreades. Some sporocarps of mycorrhizal fungi were also found in the area: Scleroderma citrina and Inocybe asterospora (Tab. 7.1). 
STUDY PLOT WOLA ŻYCIŃSKA B (5057'137N, $19^{\circ} 48^{\prime} 715 \mathrm{E}$, elevation $215.5 \mathrm{~m}$ a.s.l.)
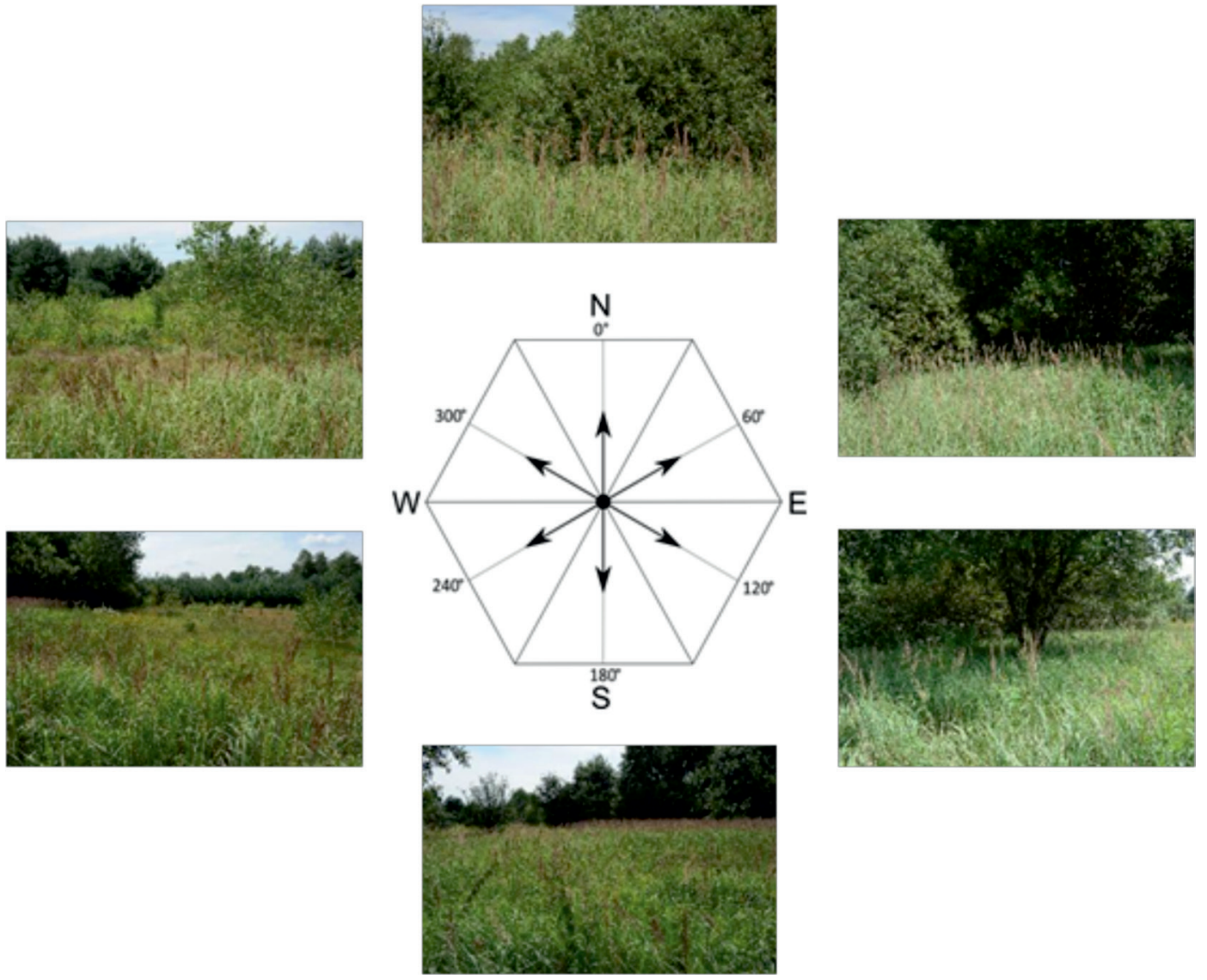

Fig. 5.23. Study plot Wola Życińska B (photo S. Krysiak, 2012)

Depth Profile description

0-30 cm - humus, fine sand with some silty sand, dark brown

30-40 cm - sandy loam, brown and yellow

40-110 cm - glacial till, brown and grey

Table 5.14. Study plot Wola Życińska B. Granulometric and chemical properties of soil

\begin{tabular}{|c|c|c|c|c|c|c|c|c|c|c|c|c|}
\hline \multirow[b]{2}{*}{ Soil pit } & \multirow{2}{*}{$\begin{array}{c}\text { Sampling } \\
\text { depth } \\
\mathrm{cm}\end{array}$} & \multicolumn{9}{|c|}{$\begin{array}{c}\text { Grain size distribution } \\
\%\end{array}$} & \multirow{2}{*}{$\begin{array}{l}\text { Gra- } \\
\text { nulo- } \\
\text { metric } \\
\text { sub- } \\
\text { group }\end{array}$} & \multirow{2}{*}{$\begin{array}{c}\text { Specific } \\
\text { surface } \\
\text { area } \\
\mathrm{m}^{2} \cdot \mathrm{g}^{-1}\end{array}$} \\
\hline & & $\begin{array}{c}2.0- \\
1.0 \\
\mathrm{~mm}\end{array}$ & $\begin{array}{c}1.0- \\
0.5 \\
\mathrm{~mm}\end{array}$ & $\begin{array}{l}0.5- \\
0.25 \\
\mathrm{~mm}\end{array}$ & $\begin{array}{c}0.25- \\
0.1 \\
\mathrm{~mm}\end{array}$ & $\begin{array}{l}0.1- \\
0.05 \\
\mathrm{~mm}\end{array}$ & $\begin{array}{c}0.05- \\
0.02 \\
\mathrm{~mm}\end{array}$ & $\begin{array}{c}0.02- \\
0.005 \\
\mathrm{~mm}\end{array}$ & $\begin{array}{c}0.005- \\
0.002 \\
\mathrm{~mm}\end{array}$ & $\begin{array}{c}<0.002 \\
\mathrm{~mm}\end{array}$ & & \\
\hline $\begin{array}{l}\text { W. Życiń- } \\
\text { ska B }\end{array}$ & $0-20$ & 1.4 & 16.6 & 28.2 & 17.2 & 9.3 & 13.9 & 9.3 & 2.4 & 1.8 & pg & 0.258 \\
\hline $\begin{array}{l}\text { W. Życiń- } \\
\text { ska B }\end{array}$ & $20-40$ & 0.0 & 9.8 & 23.2 & 16.8 & 9.8 & 17.9 & 14.5 & 4.7 & 3.3 & gp & 0.441 \\
\hline $\begin{array}{l}\text { W. Życiń- } \\
\text { ska B }\end{array}$ & $90-110$ & 0.0 & 4.9 & 16.7 & 20.9 & 8.1 & 11.7 & 18.5 & 11.4 & 7.8 & gz & 0.850 \\
\hline
\end{tabular}


Table 5.14. Study plot Wola Życińska B. Granulometric and chemical properties of soil (continued)

\begin{tabular}{|c|c|c|c|c|c|c|c|c|c|c|}
\hline Soil pit & $\begin{array}{c}\text { Sampling } \\
\text { depth } \\
\mathrm{cm}\end{array}$ & $\begin{array}{c}\text { Humus } \\
\%\end{array}$ & $\begin{array}{c}\text { C total } \\
\%\end{array}$ & $\begin{array}{c}\text { N total } \\
\%\end{array}$ & $\mathrm{C} / \mathrm{N}$ & \multicolumn{3}{|c|}{$\begin{array}{c}\mathrm{pH} \\
\mathrm{in}\end{array}$} & \multicolumn{3}{|c|}{$\begin{array}{c}\text { Available nutrients } \\
\mathrm{mg} / 100 \mathrm{~g}\end{array}$} \\
\cline { 6 - 11 } \\
$\begin{array}{c}\text { W. Życiń- } \\
\text { ska B }\end{array}$ & $0-20$ & 3.15 & 1.827 & 0.162 & 11.28 & 4.6 & 5.4 & 0.2 & 5.4 & 3.2 \\
\hline $\begin{array}{c}\text { W. Życiń- } \\
\text { ska B }\end{array}$ & $20-40$ & - & - & - & - & 4.6 & 5.5 & 0.2 & 2.9 & 3.0 \\
\hline $\begin{array}{c}\text { W. Życiń- } \\
\text { ska B }\end{array}$ & $90-110$ & - & - & - & - & 4.0 & 4.9 & 0.7 & 5.1 & 6.2 \\
\hline
\end{tabular}

\begin{tabular}{|c|c|c|c|c|c|c|c|c|c|c|}
\hline & \multirow{2}{*}{ Sampling } & \multirow{2}{*}{$\begin{array}{c}\text { Hydrolytic } \\
\text { acidity } \\
\text { depth } \\
\mathrm{cm}\end{array}$} & \multicolumn{4}{|c|}{$\begin{array}{c}\text { Exchangeable cations } \\
\mathrm{mm} / 100 \mathrm{~g}\end{array}$} & \multicolumn{4}{c|}{$\begin{array}{c}\text { Sorption capacity } \\
\mathrm{me} / 100 \mathrm{~g}\end{array}$} \\
\cline { 5 - 11 } & & $\mathrm{Ca}^{2+}$ & $\mathrm{Mg}^{2+}$ & $\mathrm{Na}^{+}$ & $\mathrm{K}^{+}$ & $\mathrm{S}$ & $\mathrm{T}$ & $(\mathrm{T}-\mathrm{S})$ & $\mathrm{V}, \%$ \\
\hline $\begin{array}{c}\text { W. Życín- } \\
\text { ska B }\end{array}$ & $0-20$ & 5.10 & 2.80 & 0.300 & 0.070 & 0.164 & 3.334 & 8.434 & 5.10 & 39.5 \\
\hline $\begin{array}{c}\text { W. Życiń- } \\
\text { ska B }\end{array}$ & $20-40$ & 3.30 & 2.10 & 0.283 & 0.070 & 0.102 & 2.555 & 5.555 & 3.30 & 46.0 \\
\hline $\begin{array}{c}\text { W. Życiń- } \\
\text { ska B }\end{array}$ & $90-110$ & 5.61 & 3.30 & 0.633 & 0.087 & 0.164 & 4.184 & 9.794 & 5.61 & 42.7 \\
\hline
\end{tabular}

Source: own elaboration.

\section{Characteristics of the flora and fungi - Wola Życińska B}

Approximately $100 \%$ of the study plot is covered with plants. Among plants, the dominating species is definitely Calamagrostis epigeois, which covers about $60 \%$ of the area. It is accompanied by the less abundant Eupatorium cannabinum (about 10\%) and Lysimachia vulgaris (10\%). The share of other species does not exceed 3\%. They include: Cirsium arvense, Hypericum perforatum, Equisetum arvense (Tab. 6.1).

Fungi of the plot are sparse. Only 3 species of macromycetes were observed. Among grasses, some sporocarps of Conocybe tenera, Psilocybe semiglobata and Psilocybe semilanceata (Tab. 7.1) were identified.

STUDY PLOT WOLA ŻYCIŃSKA C (5057'153N, 19²48'658E, elevation 215,5 m a.s.l.)
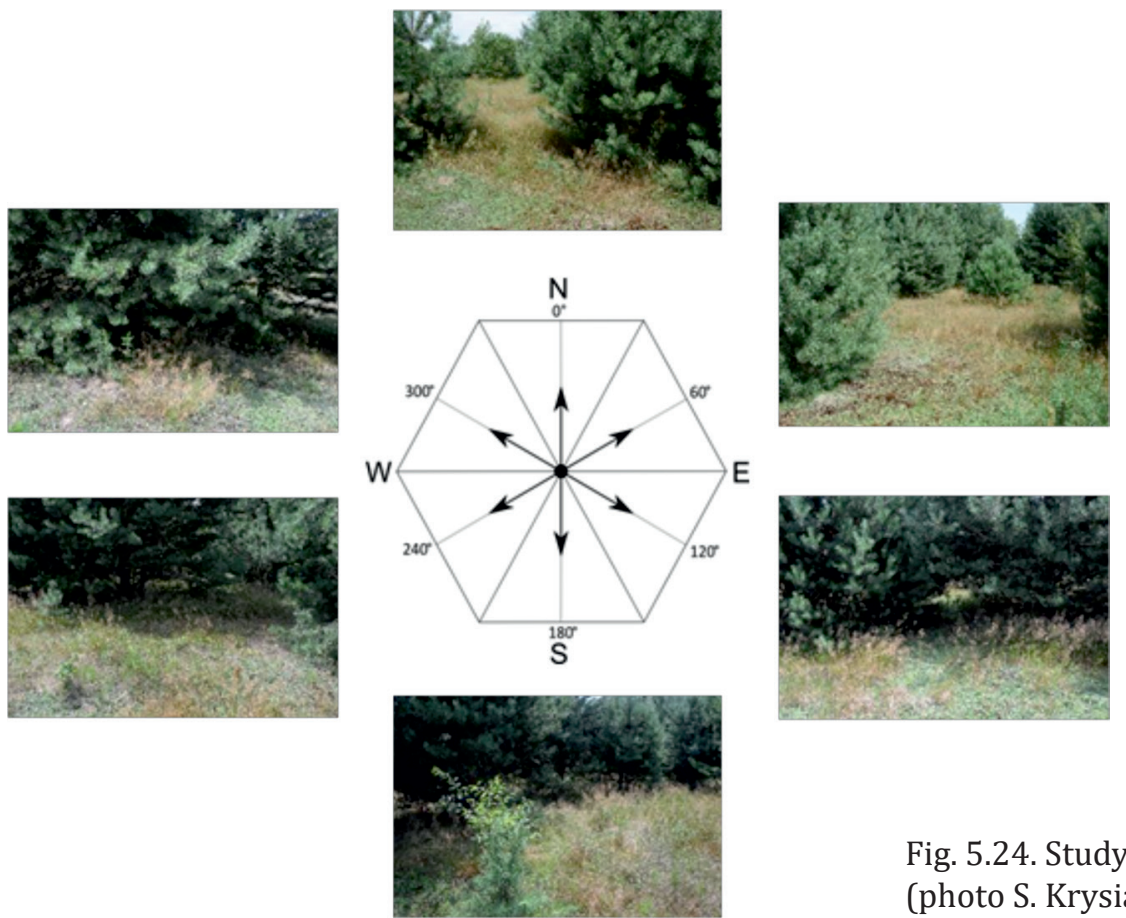

Fig. 5.24. Study plot Wola Życińska C (photo S. Krysiak, 2012) 


\section{Depth Profile description}

0-30 cm - humus, fine silty sand, grey and beige

$30-40 \mathrm{~cm}$ - fine silty sand, yellow and beige

$40-70 \mathrm{~cm}$ - fine silty sand with numerous cobbles of Scandinavian and local origin

$70-90 \mathrm{~cm}$ - fine and medium sand, yellow and brown

$90-110 \mathrm{~cm}$ - fine sand and silty sand with gravel of local origin, yellow

Table 5.15. Study plot Wola Życińska C. Granulometric and chemical properties of soil

\begin{tabular}{|c|c|c|c|c|c|c|c|c|c|c|c|c|}
\hline \multirow[b]{2}{*}{ Soil pit } & \multirow{2}{*}{$\begin{array}{c}\text { Sampling } \\
\text { depth } \\
\text { cm }\end{array}$} & \multicolumn{9}{|c|}{$\begin{array}{c}\text { Grain size distribution } \\
\%\end{array}$} & \multirow{2}{*}{$\begin{array}{l}\text { Gra- } \\
\text { nulo- } \\
\text { metric } \\
\text { sub- } \\
\text { group }\end{array}$} & \multirow{2}{*}{$\begin{array}{c}\text { Specific } \\
\text { surface } \\
\text { area } \\
\mathrm{m}^{2} \cdot \mathrm{g}^{-1}\end{array}$} \\
\hline & & $\begin{array}{c}2.0- \\
1.0 \\
\mathrm{~mm}\end{array}$ & $\begin{array}{c}1.0- \\
0.5 \\
\mathrm{~mm}\end{array}$ & $\begin{array}{l}0.5- \\
0.25 \\
\mathrm{~mm}\end{array}$ & $\begin{array}{c}0.25- \\
0.1 \\
\mathrm{~mm}\end{array}$ & $\begin{array}{l}0.1- \\
0.05 \\
\mathrm{~mm}\end{array}$ & $\begin{array}{c}0.05- \\
0.02 \\
\mathrm{~mm}\end{array}$ & $\begin{array}{c}0.02- \\
0.005 \\
\mathrm{~mm}\end{array}$ & $\begin{array}{c}0.005- \\
0.002 \\
\mathrm{~mm}\end{array}$ & $\begin{array}{c}<0.002 \\
\mathrm{~mm}\end{array}$ & & \\
\hline $\begin{array}{l}\text { W. Życiń- } \\
\text { ska C }\end{array}$ & $0-20$ & 0.1 & 15.0 & 32.9 & 27.6 & 7.9 & 8.0 & 5.9 & 1.6 & 1.1 & ps & 0.168 \\
\hline $\begin{array}{c}\text { W. Życiń- } \\
\text { ska C }\end{array}$ & $20-40$ & 0.4 & 18.9 & 32.5 & 24.6 & 6.4 & 7.1 & 6.3 & 2.2 & 1.5 & ps & 0.202 \\
\hline $\begin{array}{l}\text { W. Życiń- } \\
\text { ska C }\end{array}$ & $90-110$ & 0.1 & 14.3 & 34.7 & 41.5 & 6.4 & 0.4 & 1.4 & 0.9 & 0.2 & $\mathrm{pl}$ & 0.0622 \\
\hline $\begin{array}{l}\text { W. Życiń- } \\
\text { ska C }\end{array}$ & $130-135$ & 0.0 & 0.0 & 13.1 & 74.7 & 7.4 & 0.6 & 1.9 & 1.6 & 0.7 & $\mathrm{pl}$ & 0.1150 \\
\hline
\end{tabular}

\begin{tabular}{|c|c|c|c|c|c|c|c|c|c|c|}
\hline Soil pit & $\begin{array}{c}\text { Sampling } \\
\text { depth } \\
\mathrm{cm}\end{array}$ & $\begin{array}{c}\text { Humus } \\
\%\end{array}$ & $\begin{array}{c}\mathrm{C} \text { total } \\
\%\end{array}$ & $\begin{array}{c}\mathrm{N} \text { total } \\
\%\end{array}$ & $\mathrm{C} / \mathrm{N}$ & \multicolumn{3}{|c|}{$\begin{array}{c}\mathrm{pH} \\
\text { in }\end{array}$} & \multicolumn{3}{|c|}{$\begin{array}{c}\text { Available nutrients } \\
\mathrm{mg} / 100 \mathrm{~g}\end{array}$} \\
\cline { 6 - 11 } & & & & $\mathrm{KCl}$ & $\mathrm{H}_{2} \mathrm{O}$ & $\mathrm{P}_{2} \mathrm{O}_{5}$ & $\mathrm{~K}_{2} \mathrm{O}$ & $\mathrm{Mg}$ \\
\hline $\begin{array}{c}\text { W. Życiń- } \\
\text { ska C }\end{array}$ & $0-20$ & 1.58 & 0.916 & 0.075 & 12.21 & 4.8 & 5.8 & 9.3 & 2.6 & 0.6 \\
\hline $\begin{array}{c}\text { W. Życiń- } \\
\text { ska C }\end{array}$ & $20-40$ & - & - & - & - & 4.8 & 5.9 & 1.6 & 1.0 & 0.3 \\
\hline $\begin{array}{c}\text { W. Życiń- } \\
\text { ska C }\end{array}$ & $90-110$ & - & - & - & - & 4.3 & 5.1 & 1.0 & 0.6 & 0.3 \\
\hline $\begin{array}{c}\text { W. Życiń- } \\
\text { ska C }\end{array}$ & $130-135$ & - & - & - & - & 4.4 & 5.5 & 1.2 & 1.7 & 1.7 \\
\hline
\end{tabular}

\begin{tabular}{|c|c|c|c|c|c|c|c|c|c|c|}
\hline \multirow[t]{2}{*}{ Soil pit } & \multirow{2}{*}{$\begin{array}{c}\text { Sampling } \\
\text { depth } \\
\mathrm{cm}\end{array}$} & \multirow{2}{*}{$\begin{array}{l}\text { Hydrolytic } \\
\text { acidity } \\
\text { mmol/100g }\end{array}$} & \multicolumn{4}{|c|}{$\begin{array}{c}\text { Exchangeable cations } \\
\text { me } / 100 \mathrm{~g}\end{array}$} & \multicolumn{4}{|c|}{$\begin{array}{l}\text { Sorption capacity } \\
\text { me/100g }\end{array}$} \\
\hline & & & $\mathrm{Ca}^{2+}$ & $\mathrm{Mg}^{2+}$ & $\mathrm{Na}^{+}$ & $\mathrm{K}^{+}$ & $\mathrm{S}$ & $\mathrm{T}$ & (T-S) & $\mathrm{V}, \%$ \\
\hline $\begin{array}{l}\text { W. Życiń- } \\
\text { ska C }\end{array}$ & $0-20$ & 2.89 & 2.10 & 0.067 & 0.052 & 0.090 & 2.309 & 5.199 & 2.89 & 44.4 \\
\hline $\begin{array}{l}\text { W. Życiń- } \\
\text { ska C }\end{array}$ & $20-40$ & 1.18 & 0.80 & 0.018 & 0.026 & 0.038 & 0.882 & 2.062 & 1.18 & 42.8 \\
\hline $\begin{array}{l}\text { W. Życiń- } \\
\text { ska C }\end{array}$ & $90-110$ & 1.12 & 0.15 & 0.025 & 0.009 & 0.031 & 0.215 & 1.335 & 1.12 & 16.1 \\
\hline $\begin{array}{l}\text { W. Życiń- } \\
\text { ska C }\end{array}$ & $130-135$ & 0.90 & 0.90 & 0.155 & 0.026 & 0.044 & 1.125 & 2.025 & 0.90 & 55.5 \\
\hline
\end{tabular}

Source: own elaboration. 


\section{Characteristics of the flora and fungi - Wola Życińska C}

Approximately $80 \%$ of the study plot is covered with plants. Rich in species (26). Two species clearly dominate: Hieracium pilosella (about 50\%) and Agrostis capillaris (about 10\%). The share of other species does not exceed $1 \%$ of the area coverage. Other identified plant species include: Achillea millefolium, Elymus repens, Agrostis gigantea, Apera spica-venti, Artemisia vulgaris, Convolvulus arvensis, Dianthus carthusiana, Conyza, Hypochoeris radicata (Tab. 6.1). A few saplings of trees and shrubs also occur in the plot: Quercus robur, Crataegus monogyna and Padus serotina.
Fungi of the plot are sparse. They are represented by 7 species of macromycetes. The most frequently found one is Crinipellis scabella, which occurs on grasses, mainly on Agrostis capillaris and Festuca rubra. Among land fungi, the most abundant species was Marasmius oreades. A small number of sporocarps of mycorrhizal fungi were also found in the area: Scleroderma citrina, Amanita muscaria, Inocybe asterospora, I. corydalina and Laccaria laccata (Tab. 7.1).

\section{Analysis of granulometric composition and chemical properties of soils in the Wola Życińska study plot group}

At plots Wola Życińska A and Wola Życińska C a clear duality of sediments is visible. The upper part of the profile is formed by a periglacial cover series with $10-20 \%$ share of the silty fraction, thanks to which the sediments may be classified as the granulometric subgroups of loamy sand (pg) or slightly loamy sand (ps). The cover series are divided from the lower, more granulometrically diverse loose sands ( $\mathrm{pl}$ ), with a clear horizon of stone pavement with numerous cobbles of Scandinavian and local origin. At plot Wola Życińska B, the share of silty and clayey fraction increases with depth, which reveals a transition from the granulometric subgroup of loamy sand (pg) through sandy loam (gp) to loam (gz).

As regards agronomic classification, the granulometric composition of the humus layers at plots Wola Życińska A and B classify them with the category of light soils (category II). Slightly loamy sands in the humus layer of the plot Wola Życiń- ska C classify the soil with the category of very light soils.

At all analysed plots of the Wola Życińska study plot group, the reaction was acidic or strongly acidic. A higher content of available phosphorus $\left(\mathrm{P}_{2} \mathrm{O}_{5}\right)$, which corresponds to medium availability, was only found at plot Wola Życińska A. At all plots, the content of available potassium $\left(\mathrm{K}_{2} \mathrm{O}\right)$ was at a very low or low level. As regards the content of available magnesium, the loamy sediments of plot Wola Życińska B were above the average, reaching class II in the sample from the depth of 90-110 cm, which corresponds to high availability.

In most analysed sediments, the saturation degree of the sorptive complex with alkaline cations $\mathrm{Ca}^{2+}, \mathrm{Mg}^{2+}, \mathrm{Na}^{+}$and $\mathrm{K}^{+}$oscillated around several dozen percent. Only in the cover sediments at plot Wola Życińska A did it not exceed 10\%. 


\title{
5.2. Abandoned land study plot groups in the buffer zone around the Sieradz landscape parks
}

\author{
Elżbieta Papińska, Jolanta Adamczyk, Jarosław Sieradzki
}

\section{WERONIKA STUDY PLOT GROUP}

The described study plot group is located about $10 \mathrm{~km}$ to the east of the border of the Warta-Widawka Interfluve Landscape Park. As regards administration, the described area lies in the northern part of Zelów Commune (Bełchatów District), at the border with Buczek Commune. According to the physicogeographical division by J. Kondracki (2002), the study plot group is located within the macroregion of the South Greater Poland Lowland, in the Szczerców Basin mesoregion.

Surface sediments occurring in the Weronika study plot group include upper fluvioglacial sands deposited upon Wartanian glacial till (Baliński, Gawlik 1983). They became the parent rock for the leeched brown soils, which originated here (Województwo miejskie łódzkie. Mapa glebowo-rolnicza 1986). At plot Weronika A, the thickness of sands is the highest, which was of importance for classifying the soils originated here as complex
7 of agricultural suitability (very poor rye) and valuation class VI. At plots Weronika B and C, the thickness of sandy formations is lower, which resulted in classifying them as complex 6 of agricultural suitability (poor rye) and valuation class IVb (http://geoportal.lodzkie.pl/imap/). The current usage status of the study area is abandoned land, whose "age" was estimated at 4 to 8 years (Figures 5.25, 5.27, 5.29). All plots are located within a type 16 geocomplex, formed by plateau glacial sands and gravels upon glacial till. It belongs to the group of lithogenic geocomplexes, shaped under hydrogenic conditions associated with shallow low permeable formations. In order to document individual sites of the Weronika study plot group, photographs of soil pits were taken (Figures 5.26, 5.28, 5.30), which present the diversity of soil environment, and the results of laboratory analyses were presented in tables 5.16-5.18.

STUDY PLOT WERONIKA A (51 $28^{\prime} 5^{\prime \prime}$ N, $19^{\circ} 09^{\prime} 44^{\prime \prime}$, elevation $187 \mathrm{~m}$ a.s.l.)
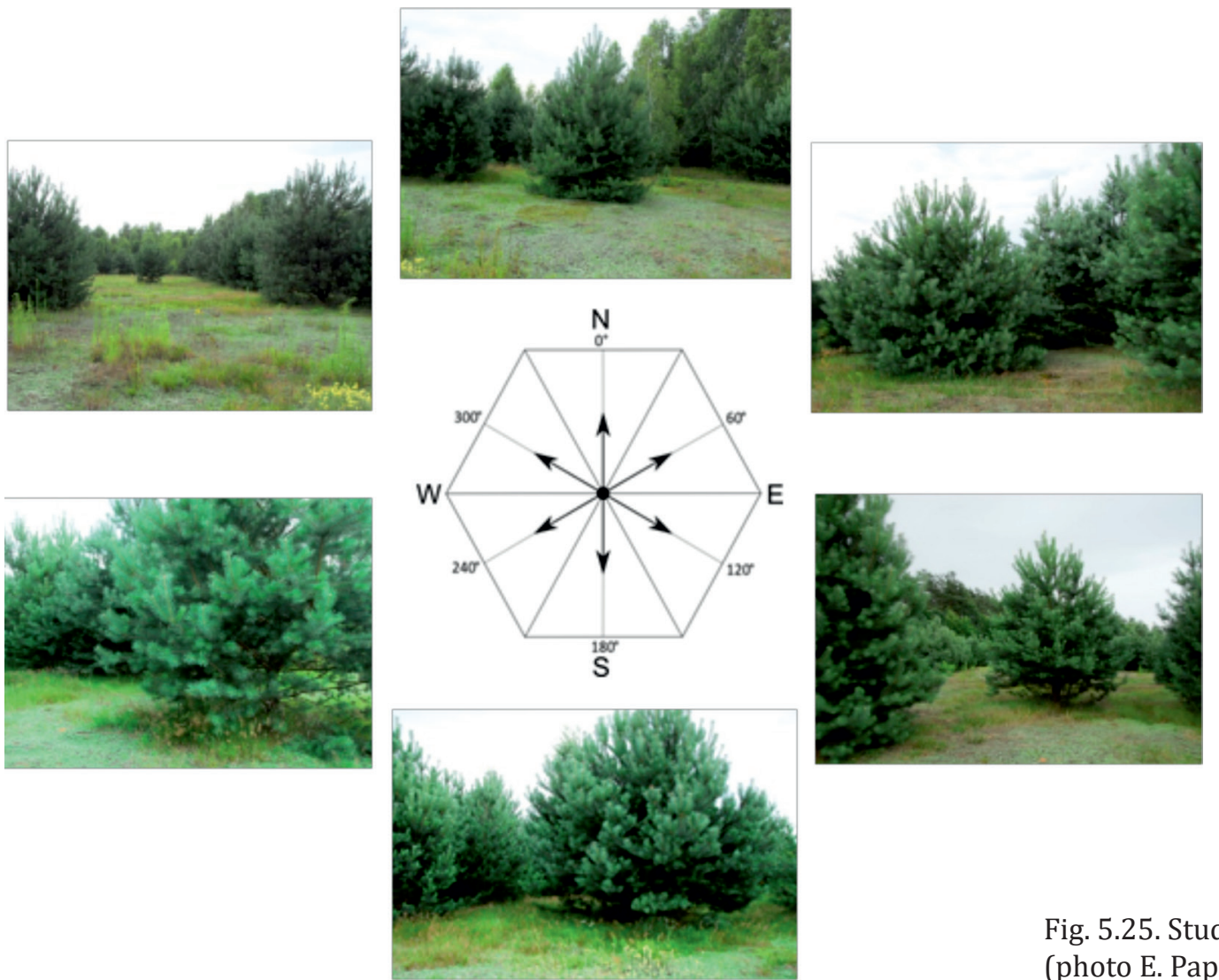

Fig. 5.25. Study plot Weronika A (photo E. Papińska, 2012) 


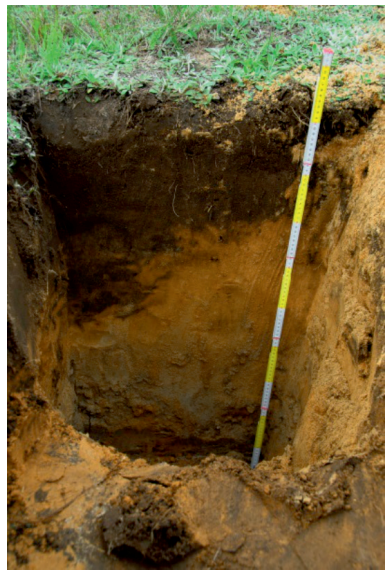

\author{
Depth Profile description \\ 0,5-0 cm - organic horizon \\ 0-25 cm - humus, coarse and medium sand, yellow \\ 25-90 cm - horizon dominated by medium sand \\ 90-100 cm - horizon with fine stones, rusty unsorted sand \\ (mostly medium) with cobbles of up to $10 \mathrm{~cm}$ \\ in diameter \\ $100-110 \mathrm{~cm}$ - horizon of parent rock, unsorted sand with rusty \\ and brown stains
}

Fig. 5.26. Soil pit at study plot Weronika A (photo E. Papińska 2012)

Table 5.16. Study plot Weronika A. Granulometric and chemical properties of soil

\begin{tabular}{|c|c|c|c|c|c|c|c|c|c|c|c|c|}
\hline \multirow[b]{2}{*}{ Soil pit } & \multirow{2}{*}{$\begin{array}{c}\text { Sampling } \\
\text { depth } \\
\text { cm }\end{array}$} & \multicolumn{9}{|c|}{$\begin{array}{c}\text { Grain size distribution } \\
\%\end{array}$} & \multirow{2}{*}{$\begin{array}{l}\text { Granu- } \\
\text { lome- } \\
\text { tric } \\
\text { sub- } \\
\text { group }\end{array}$} & \multirow{2}{*}{$\begin{array}{c}\text { Spe- } \\
\text { cific } \\
\text { surface } \\
\text { area } \\
\mathrm{m}^{2} \cdot \mathrm{g}^{-1}\end{array}$} \\
\hline & & $\begin{array}{c}2.0- \\
1.0 \\
\mathrm{~mm}\end{array}$ & $\begin{array}{c}1.0- \\
0.5 \\
\mathrm{~mm}\end{array}$ & $\begin{array}{l}0.5- \\
0.25 \\
\mathrm{~mm}\end{array}$ & $\begin{array}{c}0.25- \\
0.1 \\
\mathrm{~mm}\end{array}$ & $\begin{array}{l}0.1- \\
0.05 \\
\mathrm{~mm}\end{array}$ & $\begin{array}{c}0.05- \\
0.02 \\
\mathrm{~mm}\end{array}$ & $\begin{array}{c}0.02- \\
0.005 \\
\mathrm{~mm}\end{array}$ & $\begin{array}{c}0.005- \\
0.002 \\
\mathrm{~mm}\end{array}$ & $\begin{array}{c}<0.002 \\
\mathrm{~mm}\end{array}$ & & \\
\hline Weronika A & $0-20$ & 0.2 & 23.2 & 43.0 & 20.6 & 3.4 & 4.3 & 3.4 & 1.2 & 0.6 & $\mathrm{pl}$ & 0.1020 \\
\hline Weronika A & $20-40$ & 0.0 & 21.0 & 48.6 & 27.2 & 1.9 & 1.3 & 0.0 & 0.0 & 0.0 & $\mathrm{pl}$ & 0.0233 \\
\hline Weronika A & $90-110$ & 0.3 & 22.9 & 41.5 & 23.2 & 4.1 & 2.7 & 2.8 & 1.5 & 1.0 & $\mathrm{pl}$ & 0.1270 \\
\hline
\end{tabular}

\begin{tabular}{|c|c|c|c|c|c|c|c|c|c|c|}
\hline \multirow[t]{2}{*}{ Soil pit } & \multirow{2}{*}{$\begin{array}{l}\text { Sampling } \\
\text { depth } \\
\mathrm{cm}\end{array}$} & \multirow{2}{*}{$\begin{array}{c}\text { Humus } \\
\%\end{array}$} & \multirow{2}{*}{$\begin{array}{c}\mathrm{C} \text { total } \\
\%\end{array}$} & \multirow{2}{*}{$\begin{array}{c}\mathrm{N} \text { total } \\
\%\end{array}$} & \multirow[t]{2}{*}{$\mathrm{C} / \mathrm{N}$} & \multicolumn{2}{|c|}{$\begin{array}{l}\mathrm{pH} \\
\text { in }\end{array}$} & \multicolumn{3}{|c|}{$\begin{array}{l}\text { Available nutrients } \\
\mathrm{mg} / 100 \mathrm{~g}\end{array}$} \\
\hline & & & & & & $\mathrm{KCl}$ & $\mathrm{H}_{2} \mathrm{O}$ & $\mathrm{P}_{2} \mathrm{O}_{5}$ & $\mathrm{~K}_{2} \mathrm{O}$ & $\mathrm{Mg}$ \\
\hline Weronika A & $0-20$ & 1.73 & 1.003 & 0.062 & 12.865 & 4.2 & 5.0 & 5.8 & 1.5 & 0.5 \\
\hline Weronika A & $20-40$ & - & - & - & - & 4.7 & 5.5 & 2.5 & 0.8 & 0.4 \\
\hline Weronika A & $90-110$ & - & - & - & - & 4.5 & 5.4 & 1.4 & 0.7 & 0.9 \\
\hline
\end{tabular}

\begin{tabular}{|c|c|c|c|c|c|c|c|c|c|c|}
\hline & \multirow{2}{*}{ Soil pit } & \multirow{2}{*}{$\begin{array}{c}\text { Sempling } \\
\text { depth } \\
\mathrm{cm}\end{array}$} & \multirow{2}{*}{$\begin{array}{c}\text { Hydrolytic } \\
\text { acidity } \\
\text { mmol/100g }\end{array}$} & \multicolumn{4}{|c|}{$\begin{array}{c}\text { Exchangeable cations } \\
\mathrm{me} / 100 \mathrm{~g}\end{array}$} & \multicolumn{4}{c|}{$\begin{array}{c}\text { Sorption capacity } \\
\mathrm{me} / 100 \mathrm{~g}\end{array}$} \\
\cline { 5 - 12 } & & $\mathrm{Ca}^{2+}$ & $\mathrm{Mg}^{2+}$ & $\mathrm{Na}^{+}$ & $\mathrm{K}^{+}$ & $\mathrm{S}$ & $\mathrm{T}$ & $(\mathrm{T}-\mathrm{S})$ & $\mathrm{V}, \%$ \\
\hline Weronika A & $0-20$ & 2.84 & 0.20 & 0.042 & 0.035 & 0.056 & 0.333 & 3.173 & 2.84 & 10.495 \\
\hline Weronika A & $20-40$ & 1.22 & 0.15 & 0.025 & 0.026 & 0.026 & 0.227 & 1.447 & 1.22 & 15.688 \\
\hline Weronika A & $90-110$ & 1.08 & 0.35 & 0.072 & 0.017 & 0.031 & 0.470 & 1.550 & 1.08 & 30.323 \\
\hline
\end{tabular}

Source: own elaboration.

\title{
Characteristics of the flora and fungi - Weronika A
}

Approximately $100 \%$ of the study plot is covered with plants. Fairly rich in species (20). Two species dominate: Hieracium pilosella (about 60\%) and Chelichrysum arenaria (about 20\%) (Photo 6.3). The share of other species ranges from $3 \%$ to $0.5 \%$ of area coverage. They include: Achillea millefolium, Anthoxathum aristatum, Apera spica-venti, Convolvulus arvensis, Dactylis glomerata, Elymus repens,
Erigeron annuus, Euphorbia cyparissias. The area also features some specimens of Pinus sylvestris (Tab. 6.1).

Fungi of the plot are represented by 13 species of macromycetes. The most frequently found ones are saprobionts: Marasmius oreades and Bovista plumbea. Mycorrhizal fungi are represented by: Boletus edulis and Chalciporus piperatus (Tab. 7.1). 
STUDY PLOT WERONIKA B $\left(51^{\circ} 28^{\prime} 36^{\prime \prime} \mathrm{N}, 1^{\circ} 09^{\prime} 42^{\prime \prime}\right.$, elevation 190 m a.s.l.
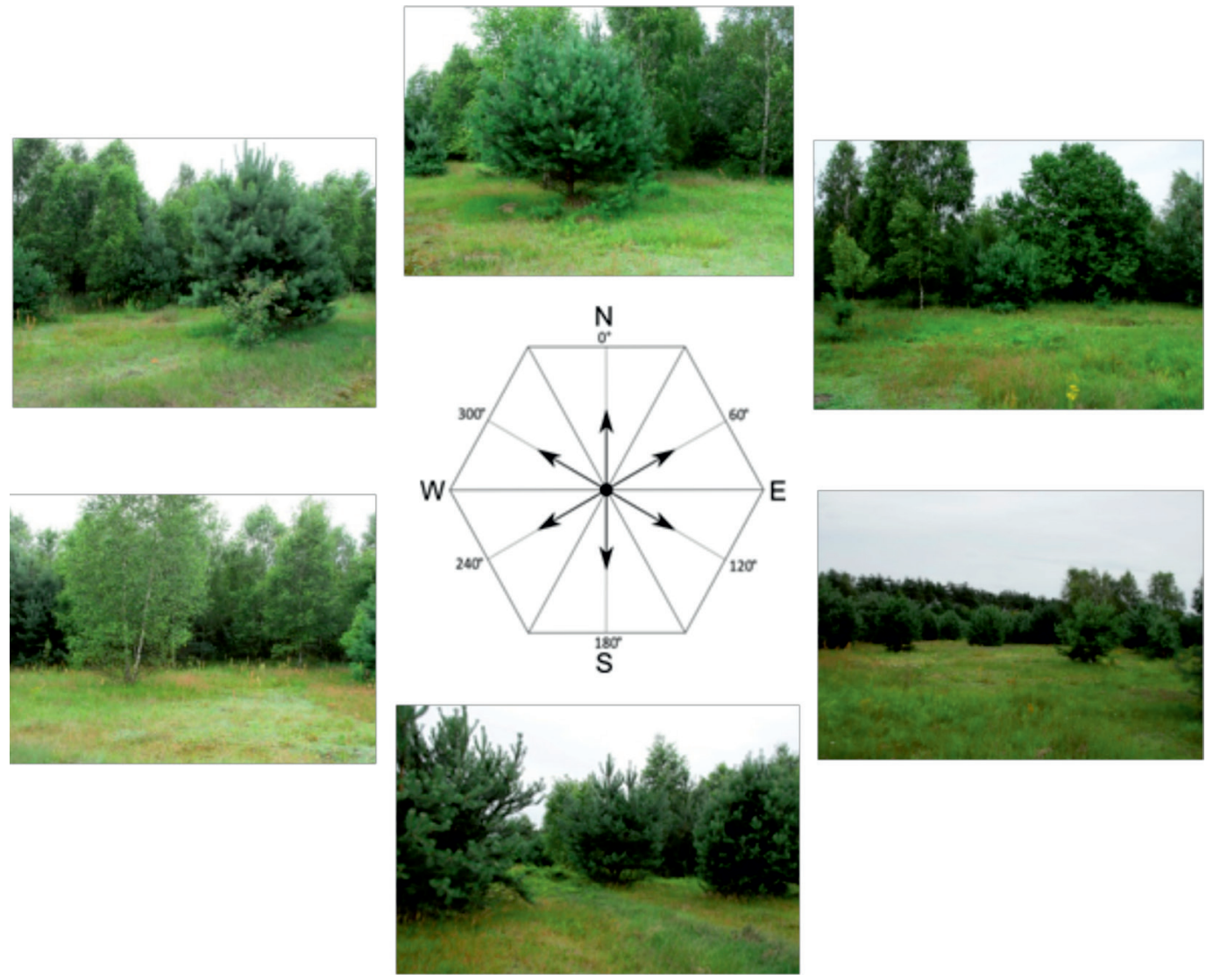

Fig. 5.27. Study plot Weronika B (photo E. Papińska, 2012)

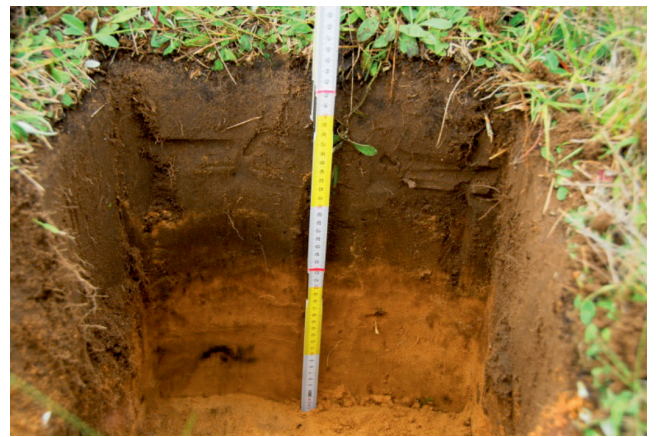

Depth Profile description

0,5-0 cm - organic horizon

0-28 cm - humus, transformed, deepened by agricultural use (ploughing) coarse and medium sand, rusty

28-90 cm - horizon with dominating medium sand and single pebbles,

90-110 cm - loamy sand turning into grey loam

Fig. 5.28. Soil pit in study plot Weronika B (photo. E. Papińska, 2012)

Table 5.17. Study plot Weronika B. Granulometric and chemical properties of soil

\begin{tabular}{|c|c|c|c|c|c|c|c|c|c|c|c|c|}
\hline \multirow[b]{2}{*}{ Soil pit } & \multirow{2}{*}{$\begin{array}{c}\text { Sampling } \\
\text { depth } \\
\text { cm }\end{array}$} & \multicolumn{9}{|c|}{$\begin{array}{c}\text { Grain size distribution } \\
\% \\
\end{array}$} & \multirow{2}{*}{$\begin{array}{c}\text { Granu- } \\
\text { lome- } \\
\text { tric } \\
\text { sub- } \\
\text { group }\end{array}$} & \multirow{2}{*}{$\begin{array}{c}\text { Spe- } \\
\text { cific } \\
\text { surface } \\
\text { area } \\
\mathrm{m}^{2} \cdot \mathrm{g}^{-1}\end{array}$} \\
\hline & & $\begin{array}{c}2.0- \\
1.0 \\
\mathrm{~mm} \\
\end{array}$ & $\begin{array}{c}1.0- \\
0.5 \\
\mathrm{~mm}\end{array}$ & $\begin{array}{l}0.5- \\
0.25 \\
\mathrm{~mm} \\
\end{array}$ & $\begin{array}{c}0.25- \\
0.1 \\
\mathrm{~mm}\end{array}$ & $\begin{array}{l}0.1- \\
0.05 \\
\mathrm{~mm} \\
\end{array}$ & $\begin{array}{c}0.05- \\
0.02 \\
\mathrm{~mm}\end{array}$ & $\begin{array}{c}0.02- \\
0.005 \\
\mathrm{~mm}\end{array}$ & $\begin{array}{c}0.005- \\
0.002 \\
\mathrm{~mm} \\
\end{array}$ & $\begin{array}{c}<0.002 \\
\mathrm{~mm}\end{array}$ & & \\
\hline Weronika B & $0-20$ & 0.2 & 22.4 & 42.1 & 21.0 & 3.6 & 4.5 & 3.9 & 1.4 & 0.9 & $\mathrm{pl}$ & 0.1280 \\
\hline Weronika B & $20-40$ & 0.0 & 19.7 & 48.1 & 28.4 & 2.7 & 1.2 & 0.0 & 0.0 & 0.0 & $\mathrm{pl}$ & 0.0239 \\
\hline Weronika B & $90-110$ & 0.3 & 19.0 & 33.2 & 20.9 & 5.0 & 4.3 & 8.0 & 5.4 & 4.0 & pg & 0.4280 \\
\hline
\end{tabular}


Table 5.17. Study plot Weronika B. Granulometric and chemical properties of soil (continued)

\begin{tabular}{|c|c|c|c|c|c|c|c|c|c|c|}
\hline \multirow{2}{*}{ Soil pit } & $\begin{array}{c}\text { Sampling } \\
\text { depth } \\
\end{array}$ & $\begin{array}{c}\text { Humus } \\
\text { cm }\end{array}$ & \multirow{2}{*}{$\begin{array}{c}\text { C total } \\
\%\end{array}$} & \multirow{2}{*}{$\begin{array}{c}\mathrm{N} \text { total } \\
\%\end{array}$} & \multirow{2}{*}{$\mathrm{C} / \mathrm{N}$} & \multicolumn{3}{|c|}{$\begin{array}{c}\mathrm{pH} \\
\text { in }\end{array}$} & \multicolumn{3}{|c|}{$\begin{array}{c}\text { Available nutrients } \\
\mathrm{mg} / 100 \mathrm{~g}\end{array}$} \\
\cline { 6 - 11 } & & & & $\mathrm{KCl}$ & $\mathrm{H}_{2} \mathrm{O}$ & $\mathrm{P}_{2} \mathrm{O}_{5}$ & $\mathrm{~K}_{2} \mathrm{O}$ & $\mathrm{Mg}$ \\
\hline Weronika B & $0-20$ & 1.33 & 0.771 & 0.051 & 15.127 & 4.1 & 4.9 & 8.0 & 3.2 & 0.6 \\
\hline Weronika B & $20-40$ & - & - & - & - & 4.6 & 4.9 & 3.4 & 0.6 & 0.3 \\
\hline Weronika B & $90-110$ & - & - & - & - & 4.3 & 5.2 & 1.5 & 2.2 & 3.0 \\
\hline
\end{tabular}

\begin{tabular}{|c|c|c|c|c|c|c|c|c|c|c|}
\hline \multirow{2}{*}{ Soil pit } & \multirow{2}{*}{$\begin{array}{c}\text { Sampling } \\
\text { depth }\end{array}$} & \multirow{2}{*}{$\begin{array}{c}\text { Hydrolytic } \\
\text { acidity } \\
\mathrm{mmol} / 100 \mathrm{~g}\end{array}$} & \multicolumn{4}{|c|}{$\begin{array}{c}\text { Exchangeable cations } \\
\mathrm{me} / 100 \mathrm{~g}\end{array}$} & \multicolumn{4}{c|}{$\begin{array}{c}\text { Sorption capacity } \\
\mathrm{me} / 100 \mathrm{~g}\end{array}$} \\
\hline & & $\mathrm{Ca}^{2+}$ & $\mathrm{Mg}^{2+}$ & $\mathrm{Na}^{+}$ & $\mathrm{K}^{+}$ & $\mathrm{S}$ & $\mathrm{T}$ & $(\mathrm{T}-\mathrm{S})$ & $\mathrm{V}, \%$ \\
\hline Weronika B & $0-20$ & 3.23 & 0.15 & 0.052 & 0.026 & 0.102 & 0.330 & 3.560 & 3.23 & 9.270 \\
\hline Weronika B & $20-40$ & 1.38 & 0.10 & 0.015 & 0.009 & 0.026 & 0.150 & 1.530 & 1.38 & 9.804 \\
\hline Weronika B & $90-110$ & 1.75 & 1.00 & 0.280 & 0.043 & 0.079 & 1.402 & 3.152 & 1.75 & 44.480 \\
\hline
\end{tabular}

Source: own elaboration.

\section{Characteristics of the flora and fungi - Weronika B}

Approximately $80 \%$ of the study plot is covered with plants. Fairly rich in species (21). Two species dominate: Hieracium pilosella (about 35\%) and Agrostis capillaris (about 20\%). The share of other species ranges from $5 \%$ to $0.5 \%$ of area coverage. Other identified plant species include: Achillea millefolium, Chenopodium album, Convolvulus arvensis, Dactylis glomerata, Euphorbia cyparissias, Equise- tum arvense, Equisetum sylvaticum. The area also features specimens of trees: Pinus sylvesris, Pyrus communis, Sorbus aucuparia (Tab. 6.1).

Fungi of the plot include 11 species of macromycetes. Frequently found ones include: Bovista pumbea, Amanita muscaria, Boletus edulis. Dead pine wood is inhabited by Schizophyllum commune.

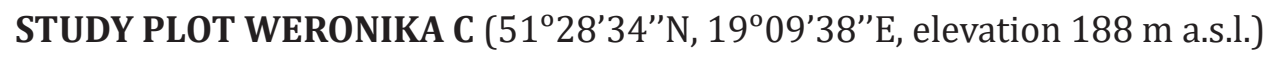
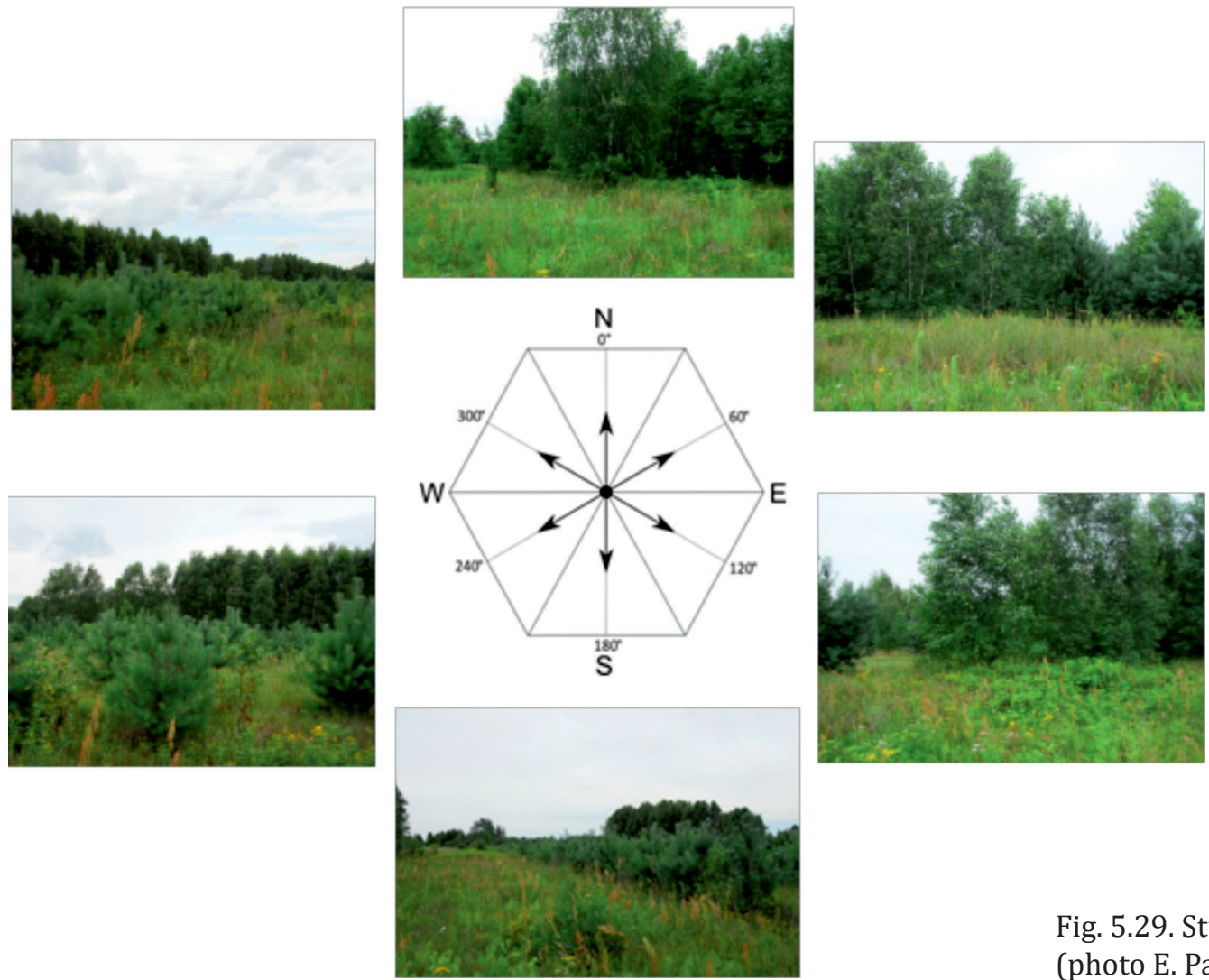

Fig. 5.29. Study plot Weronika C (photo E. Papińska, 2012) 


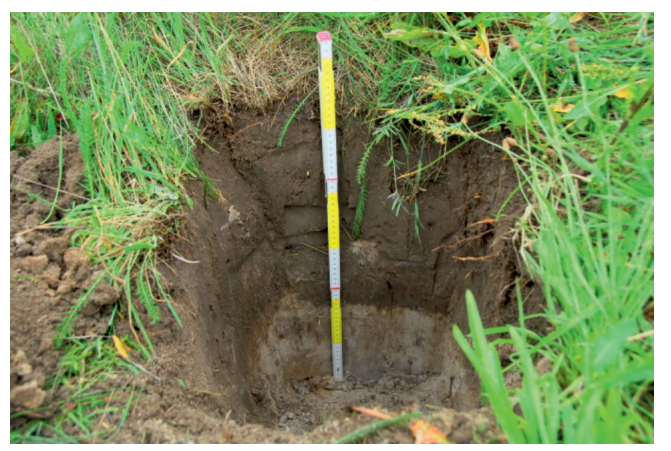

\author{
Depth Profile description \\ 1-0 cm - organic horizon \\ 0-37 cm - humus, transformed and deepened by agricultu- \\ ral use (ploughing) \\ $37-75 \mathrm{~cm}$ - coarse and medium sand with dominating light \\ grey sand \\ $75-110 \mathrm{~cm}$ - grey loam
}

Fig. 5.30. Soil pit in study plot Weronika C (photo. E. Papińska, 2012)

Table 5.18. Study plot Weronika C. Granulometric and chemical properties of soil

\begin{tabular}{|c|c|c|c|c|c|c|c|c|c|c|c|c|}
\hline \multirow[b]{2}{*}{ Soil pit } & \multirow{2}{*}{$\begin{array}{c}\text { Sampling } \\
\text { depth } \\
\mathrm{cm}\end{array}$} & \multicolumn{9}{|c|}{$\begin{array}{c}\text { Grain size distribution } \\
\%\end{array}$} & \multirow{2}{*}{$\begin{array}{l}\text { Granu- } \\
\text { lome- } \\
\text { tric } \\
\text { sub- } \\
\text { group }\end{array}$} & \multirow{2}{*}{$\begin{array}{c}\text { Spe- } \\
\text { cific } \\
\text { surface } \\
\text { area } \\
\mathrm{m}^{2} \cdot \mathrm{g}-1\end{array}$} \\
\hline & & $\begin{array}{c}2.0- \\
1.0 \\
\mathrm{~mm}\end{array}$ & $\begin{array}{c}1.0- \\
0.5 \\
\mathrm{~mm}\end{array}$ & $\begin{array}{l}0.5- \\
0.25 \\
\mathrm{~mm}\end{array}$ & $\begin{array}{c}0.25- \\
0.1 \\
\mathrm{~mm}\end{array}$ & $\begin{array}{l}0.1- \\
0.05 \\
\mathrm{~mm}\end{array}$ & $\begin{array}{c}0.05- \\
0.02 \\
\mathrm{~mm}\end{array}$ & $\begin{array}{c}0.02- \\
0.005 \\
\mathrm{~mm}\end{array}$ & $\begin{array}{c}0.005- \\
0.002 \\
\mathrm{~mm}\end{array}$ & $\begin{array}{c}<0.002 \\
\mathrm{~mm}\end{array}$ & & \\
\hline Weronika C & $0-20$ & 0.9 & 26.0 & 40.9 & 18.4 & 3.6 & 4.6 & 3.8 & 1.1 & 0.6 & $\mathrm{pl}$ & 0.103 \\
\hline Weronika C & $20-40$ & 1.1 & 24.7 & 40.8 & 21.5 & 2.8 & 2.8 & 3.5 & 1.8 & 1.1 & $\mathrm{pl}$ & 0.138 \\
\hline Weronika C & $90-110$ & 1.8 & 12.8 & 16.8 & 15.3 & 6.1 & 8.4 & 17.9 & 12.0 & 8.8 & gl & 0.915 \\
\hline
\end{tabular}

\begin{tabular}{|c|c|c|c|c|c|c|c|c|c|c|}
\hline \multirow[t]{2}{*}{ Soil pit } & \multirow{2}{*}{$\begin{array}{c}\text { Sampling } \\
\text { depth } \\
\text { cm }\end{array}$} & \multirow{2}{*}{$\begin{array}{c}\text { Humus } \\
\%\end{array}$} & \multirow{2}{*}{$\begin{array}{l}\text { C total } \\
\%\end{array}$} & \multirow{2}{*}{$\begin{array}{l}\mathrm{N} \text { total } \\
\%\end{array}$} & \multirow[t]{2}{*}{$\mathrm{C} / \mathrm{N}$} & \multicolumn{2}{|c|}{$\begin{array}{l}\mathrm{pH} \\
\text { in }\end{array}$} & \multicolumn{3}{|c|}{$\begin{array}{c}\text { Available nutrients } \\
\mathrm{mg} / 100 \mathrm{~g}\end{array}$} \\
\hline & & & & & & $\mathrm{KCl}$ & $\mathrm{H}_{2} \mathrm{O}$ & $\mathrm{P}_{2} \mathrm{O}_{5}$ & $\mathrm{~K}_{2} \mathrm{O}$ & $\mathrm{Mg}$ \\
\hline Weronika C & $0-20$ & 1.02 & 0.592 & 0.058 & 10.201 & 4.5 & 5.5 & 5.2 & 3.8 & 0.7 \\
\hline Weronika C & $20-40$ & - & - & - & - & 4.8 & 6.0 & 1.9 & 1.3 & 1.1 \\
\hline Weronika C & $90-110$ & - & - & - & - & 4.1 & 5.4 & 3.0 & 5.8 & 20.0 \\
\hline
\end{tabular}

\begin{tabular}{|c|c|c|c|c|c|c|c|c|c|c|}
\hline & \multirow{2}{*}{ Soil pit } & \multirow{2}{*}{$\begin{array}{c}\text { Sampling } \\
\text { depth } \\
\mathrm{cm}\end{array}$} & \multirow{2}{*}{$\begin{array}{c}\text { Hydrolytic } \\
\text { acidity } \\
\mathrm{mmol} / 100 \mathrm{~g}\end{array}$} & \multicolumn{4}{|c|}{$\begin{array}{c}\text { Exchangeable cations } \\
\mathrm{me} / 100 \mathrm{~g}\end{array}$} & \multicolumn{4}{c|}{$\begin{array}{c}\text { Sorption capacity } \\
\text { me/100g }\end{array}$} \\
\cline { 5 - 13 } & & $\mathrm{Ca}^{2+}$ & $\mathrm{Mg}^{2+}$ & $\mathrm{Na}^{+}$ & $\mathrm{K}^{+}$ & $\mathrm{S}$ & $\mathrm{T}$ & $(\mathrm{T}-\mathrm{S})$ & $\mathrm{V}, \%$ \\
\hline Weronika C & $0-20$ & 2.37 & 1.05 & 0.053 & 0.043 & 0.120 & 1.266 & 3.636 & 2.37 & 34.818 \\
\hline Weronika C & $20-40$ & 0.84 & 0.65 & 0.092 & 0.035 & 0.044 & 0.821 & 1.661 & 0.84 & 49.428 \\
\hline Weronika C & $90-110$ & 2.68 & 6.20 & 2.220 & 0.209 & 0.208 & 8.837 & 11.517 & 2.68 & 76.730 \\
\hline
\end{tabular}

Source: own elaboration.

\title{
Characteristics of the flora and fungi - Weronika C
}

Approximately $80 \%$ of the study plot is covered with plants. Fairly rich in species (25). Two species dominate: Agrostis capillaris (about 20\%) and Rumex acetosa (10\%). The share of other species ranges from $7 \%$ to $0.5 \%$ of area coverage. They include: Arrthenatherum elatius, Conyza canadensis, Dactylis glomerata, Cirsium arvense, Equisetum sylvaticum, Hieracium pilosella, Hieracium umbellatum, Holcus lanatus, Juncus effusus.
The area also features specimens of trees and shrubs: Pinus sylvestris, Pyrus communis, Padus serotina and Frangula alnus (Tab. 6.1).

Fungi of the plot are not too abundant. They are represented by 10 species of macromycetes (Photo 7.1). The frequently found species include: Entoloma conferendum, Macrolepiota procera and mycorrhizal species: Amanita muscaria and Boletus edulis (Tab. 7.1). 


\section{Analysis of granulometric composition and chemical properties of soils in the Weronika Study Plot Group}

A comparison of grain size distribution results for individual plots of the Weronika study group (Tab. 5.16-5.18) clearly indicates the dominance of the sandy fraction (Polskie Towarzystwo Gleboznawcze 2009) in all horizons at plot Weronika A and in two horizons $(0-20 \mathrm{~cm}$ and $20-40 \mathrm{~cm})$ at plots B and C. The share of this fraction in these horizons ranges from 90 to $99 \%$. The medium sand fraction dominates, reaching $40-48 \%$ in samples from the depth of $20-40 \mathrm{~cm}$ at all sites. The share of coarse and fine sands is similar and oscillates around $18-26 \%$ in two horizons $(0-20 \mathrm{~cm}$ and $20-40 \mathrm{~cm})$ at all sites. The situation begins to change at the deepest analysed depth at plots B and C. At plot B, the share of total sandy fraction in horizon $90-100 \mathrm{~cm}$ is below $80 \%$, and it is still lower at plot $\mathrm{C}$, where it drops to about $53 \%$. Simultaneously, the share of silty fraction in this horizon at both plots increases to $17.7 \%$ at plot $\mathrm{B}$ and up to $38.3 \%$ at plot $\mathrm{C}$. There is a clearly increased share of clay in this horizon - up to $4 \%$ at plot $\mathrm{B}$ and $8.8 \%$ at plot $\mathrm{C}$. The results of granulometric analyses of samples from individual horizons indicate fairly subtle differences between plots. At plot Weronika A, the granulometric subgroup of loose sand ( $\mathrm{pl}$ ) was determined for all horizons. At plot Weronika B, samples from two horizons $(0-20 \mathrm{~cm}$ and $20-40 \mathrm{~cm})$ were classified as loose sands (pl), whereas the sample from the depth of 90-100 cm - as loamy sand (pg). At plot Weronika $\mathrm{C}$, the situation is similar, except that the deepest sample exhibits the properties of sandy loam (gp).

All analysed soils in the Weronika study plot group were categorised agronomically. Samples classified as loose sands on the basis of their grain size distribution are classified as agronomic category I (very light soils). Another parameter which characterises the properties of soils at the described sites - specific surface area - is dependent on grain size distribution. Data included in tables 5.16-5.18 clearly shows that it is at its lowest in these horizons, where the share of the sandy fraction is close to $100 \%$. Such a situation occurs at plots Weronika A and B in samples from the depth of 20-40 cm, whereas the specific surface area value is $0.0233 \mathrm{~m}^{2} \cdot \mathrm{g}^{-1}$ (Weronika A) and 0.0239 $\mathrm{m}^{2} \cdot \mathrm{g}-1$ (Weronika $\mathrm{B}$ ). The value of this parameter increases with the increase of the percentage of silts and clays in the sample, which can be observed at plot Weronika $\mathrm{C}$ in the sample from the depth 90-100 cm, where it reaches the maximum of $0.915 \mathrm{~m}^{2} \cdot \mathrm{g}^{-1}$, with the content of both mentioned fractions at nearly $40 \%$.

The $\mathrm{pH}$ reaction in $\mathrm{KCl}$ in all samples from all three plots is acidic and very acidic and ranges from 4.1 (Weronika B, sample 0-20 cm) to 4.8 (Weronika C, sample $20-40 \mathrm{~cm}$ ). The highest values are recorded in samples taken from the depth of $20-40 \mathrm{~cm}$. The $\mathrm{pH}$ reaction in $\mathrm{H}_{2} \mathrm{O}$ ranges from 4.9 to 6 . The highest $\mathrm{pH}$ value of 6 was found in the sample from the depth of 20-40 $\mathrm{cm}$ at plot Weronika $\mathrm{C}$, which is also reflected in the high level of saturation with alkaline cations (V), which is $76.73 \%$. The lowest $\mathrm{pH}$ value was found at plot Weronika $B$ in samples from the depth of $0-20 \mathrm{~cm}$ and $20-40 \mathrm{~cm}$, where the saturation with alkaline cations is the lowest and is $9.27 \%$ and $9.804 \%$, respectively. Low values of this parameter make the soil susceptible to fluctuations of the reaction and chemical degradation caused by acidic substances. In such events, the buffering capacity of the soil is low. Assessing the content of individual nutrients available for plants, it may be stated that for phosphorus $\left(\mathrm{P}_{2} \mathrm{O}_{5}\right)$ it changes from low in the $0-20 \mathrm{~cm}$ horizon at all sites, to very low in the other two samples for all horizons. The content of potassium $\left(\mathrm{K}_{2} \mathrm{O}\right)$ is very low and low (only in 2 samples), and the content of magnesium (Mg) in most horizons is very low and low. Only the content in the sample from the depth of $90-100 \mathrm{~cm}$ at plot Weronika C falls within the very high availability class.

The content of humus in the $0-20 \mathrm{~cm}$ horizon is the lowest at plot Weronika $\mathrm{A}-0.95 \%$, and the highest at plot Weronika B $-1.33 \%$. The content of organic carbon (C) is fairly high at plot Weronika B $-0.771 \%$. At the two other plots, the content is lower and falls below $0.6 \%$. 


\section{WOLA PSZCZÓŁECKA STUDY PLOT GROUP}

The described study plot group is located at the elevation of 165-170 $\mathrm{m}$ a.s.l., on the border between the Łask Plateau and the Szczerców Basin (Kondracki 2002), about $8.5 \mathrm{~km}$ to the east of the border of the Warta-Widawka Interfluve Landscape Park, in Zelów Commune. The study plot group lies within the terrace of the Chrzęstawka, a right tributary of Widawka. According to the Detailed Geological Map of Poland, scale 1:50 000, sheet Zelów (Baliński, Gawlik 1983), Vistulian fluvial sands of terraces upon Wartanian loams occur here. On the 1:5000 soil and agricultural map, within the analysed area there are leeched brown soils (Bw), agricultural suitability class 7 (very poor rye), developed upon loose sands (pl) at plots Wola Pszczółecka A and B. The soils were qualified as valuation class VI (http:// geoportal.lodzkie.pl/imap/). At plot Wola Pszczółecka $C$, the map shows podzolic soils (A), of agricultural suitability class 5 (good rye), developed upon slightly loamy sands (psg) upon sandy loams (gp). The soils were qualified as valuation class IVb (http://geoportal.lodzkie.pl/imap/). The current usage - abandoned land, whose "age" is estimated at a minimum of 5-6 years. The physiognomy of individual study plots within the analysed group is presented in photographs (Figures 5.31, $5.33,5.35)$. The study plot group is located within a type 8 geocomplex, which is formed by fluvial sands and gravels of upper terraces. Similarly to the previous case, soil pits were excavated (Figures 5.32, 5.34, 5.36) from which samples were taken for laboratory studies, and their results are presented in tables 5.19-5.21.

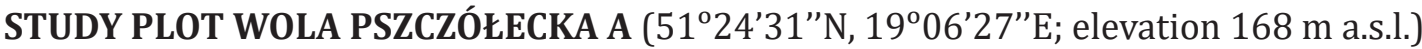
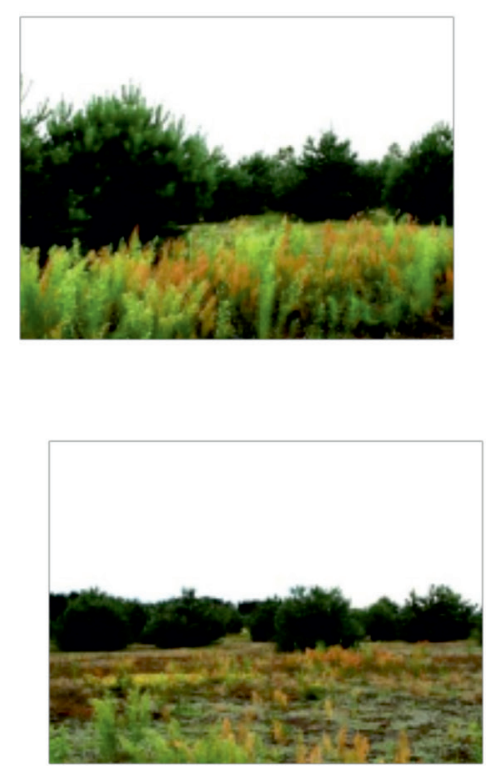
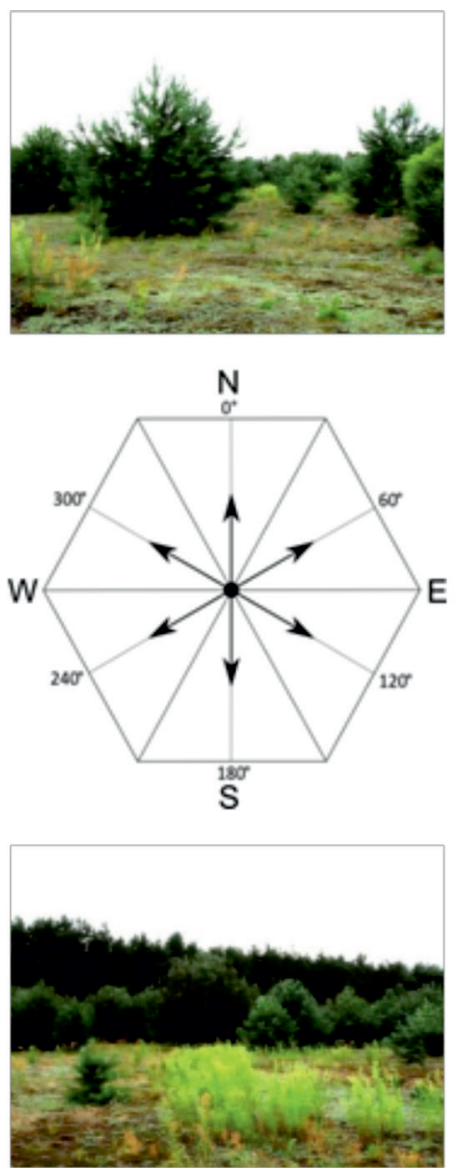
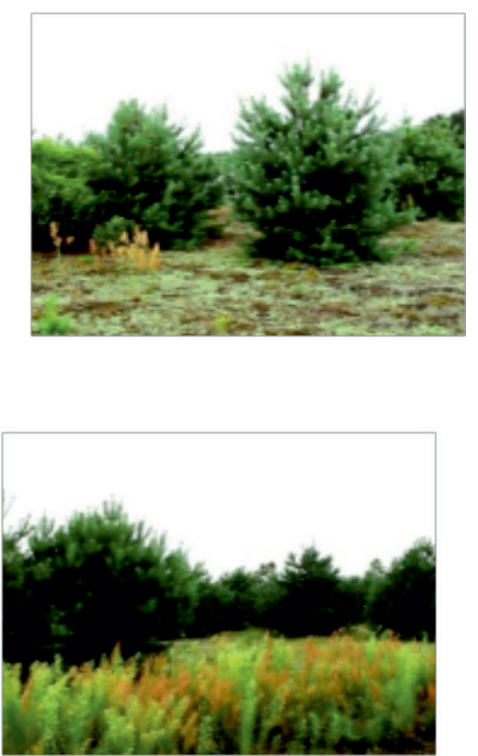

Fig. 5.31. Study plot Wola Pszczółecka A (photo E. Papińska, 2012) 


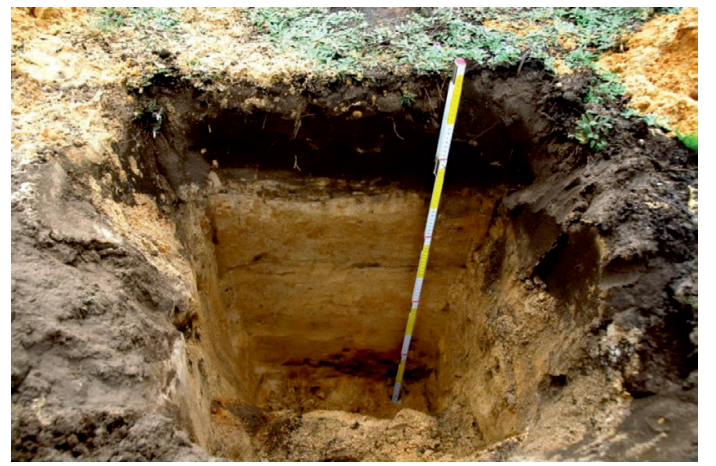

Depth Profile description

1-0 cm - organic horizon

0-25 cm - humus, transformed, deepened by agricultural use (ploughing) coarse and medium sand, grey and brown

25-30 cm - depletion layer, reduced thickness probably due to deep ploughing with dominating medium sand

30-85 cm - enrichment horizon, unsorted sand (mostly medium) light yellow

85-110 cm - horizon of parent rock, unsorted sand, rusty wit pebbles and cobbles of $8 \mathrm{~cm}$ in diameter

Fig. 5.32. Soil pit in study plot Wola Pszczółecka A (photo. E. Papińska, 2012)

Table 5.19. Study plot Wola Pszczółecka A. Granulometric and chemical properties of soil

\begin{tabular}{|c|c|c|c|c|c|c|c|c|c|c|c|c|}
\hline \multirow[b]{2}{*}{ Soil pit } & \multirow{2}{*}{$\begin{array}{c}\text { Sam- } \\
\text { pling } \\
\text { depth } \\
\text { cm }\end{array}$} & \multicolumn{9}{|c|}{$\begin{array}{c}\text { Grain size distribution } \\
\%\end{array}$} & \multirow{2}{*}{$\begin{array}{l}\text { Granu- } \\
\text { lome- } \\
\text { tric } \\
\text { sub- } \\
\text { group }\end{array}$} & \multirow{2}{*}{$\begin{array}{c}\text { Spe- } \\
\text { cific } \\
\text { surface } \\
\text { area } \\
\mathrm{m}^{2} \cdot \mathrm{g}^{-1}\end{array}$} \\
\hline & & $\begin{array}{c}2.0- \\
1.0 \\
\mathrm{~mm}\end{array}$ & $\begin{array}{c}1.0- \\
0.5 \\
\mathrm{~mm}\end{array}$ & $\begin{array}{l}0.5- \\
0.25 \\
\mathrm{~mm}\end{array}$ & $\begin{array}{c}0.25- \\
0.1 \\
\mathrm{~mm}\end{array}$ & $\begin{array}{l}0.1- \\
0.05 \\
\mathrm{~mm}\end{array}$ & $\begin{array}{c}0.05- \\
0.02 \\
\mathrm{~mm}\end{array}$ & $\begin{array}{c}0.02- \\
0.005 \\
\mathrm{~mm}\end{array}$ & $\begin{array}{c}0.005- \\
0.002 \\
\mathrm{~mm}\end{array}$ & $\begin{array}{c}<0.002 \\
\mathrm{~mm}\end{array}$ & & \\
\hline $\begin{array}{c}\text { Wola Pszczó- } \\
\text { łecka A }\end{array}$ & $0-20$ & 0.4 & 26.1 & 45.5 & 21.6 & 1.9 & 2 & 1.9 & 0.6 & 0.0 & $\mathrm{pl} \mathrm{gr}$ & 0.0462 \\
\hline $\begin{array}{c}\text { Wola Pszczó- } \\
\text { łecka A }\end{array}$ & $20-40$ & 0.0 & 22.6 & 51.7 & 25.6 & 0.1 & 0 & 0.0 & 0.0 & 0.0 & $\mathrm{pl} \mathrm{sr}$ & 0.0195 \\
\hline $\begin{array}{l}\text { Wola Pszczó- } \\
\text { łecka A }\end{array}$ & $90-110$ & 1.1 & 26.2 & 40.5 & 23.4 & 4.2 & 2 & 1.5 & 0.8 & 0.3 & pl gr & 0.0617 \\
\hline
\end{tabular}

\begin{tabular}{|c|c|c|c|c|c|c|c|c|c|c|}
\hline \multirow{2}{*}{ Soil pit } & \multirow{2}{*}{$\begin{array}{l}\text { Sam- } \\
\text { pling } \\
\text { depth } \\
\text { cm }\end{array}$} & \multirow{2}{*}{$\begin{array}{c}\text { Humus } \\
\%\end{array}$} & \multirow{2}{*}{$\begin{array}{c}\text { C total } \\
\%\end{array}$} & \multirow{2}{*}{$\begin{array}{c}\mathrm{N} \text { total } \\
\%\end{array}$} & \multirow{2}{*}{$\mathrm{C} / \mathrm{N}$} & \multicolumn{2}{|c|}{$\begin{array}{l}\mathrm{pH} \\
\text { in }\end{array}$} & \multicolumn{3}{|c|}{$\begin{array}{c}\text { Available nutrients } \\
\mathrm{mg} / 100 \mathrm{~g}\end{array}$} \\
\hline & & & & & & $\mathrm{KCl}$ & $\mathrm{H}_{2} \mathrm{O}$ & $\mathrm{P}_{2} \mathrm{O}_{5}$ & $\mathrm{~K}_{2} \mathrm{O}$ & $\mathrm{Mg}$ \\
\hline $\begin{array}{c}\text { Wola Pszczó- } \\
\text { łecka A }\end{array}$ & $0-20$ & 1.29 & 0.748 & 0.057 & 13.127 & 4.3 & 5.2 & 12.0 & 2.8 & 1.0 \\
\hline $\begin{array}{c}\text { Wola Pszczó- } \\
\text { łecka A }\end{array}$ & $20-40$ & - & - & - & - & 4.8 & 5.0 & 5.1 & 0.5 & 0.3 \\
\hline $\begin{array}{c}\text { Wola Pszczó- } \\
\text { łecka A }\end{array}$ & $90-110$ & - & - & - & - & 4.5 & 4.8 & 1.9 & 1.0 & 0.4 \\
\hline
\end{tabular}

\begin{tabular}{|c|c|c|c|c|c|c|c|c|c|c|}
\hline \multirow{2}{*}{ Soil pit } & \multirow{2}{*}{$\begin{array}{c}\text { Sam- } \\
\text { pling } \\
\text { depth } \\
\text { cm }\end{array}$} & \multirow{2}{*}{$\begin{array}{l}\text { Hydrolytic } \\
\text { acidity } \\
\text { mmol/100g }\end{array}$} & \multicolumn{4}{|c|}{$\begin{array}{c}\text { Exchangeable cations } \\
\text { me } / 100 \mathrm{~g}\end{array}$} & \multicolumn{4}{|c|}{$\begin{array}{c}\text { Sorption capacity } \\
\text { me } / 100 \mathrm{~g}\end{array}$} \\
\hline & & & $\mathrm{Ca}^{2^{+}}$ & $\mathrm{Mg}^{2+}$ & $\mathrm{Na}^{+}$ & $\mathrm{K}^{+}$ & S & $\mathrm{T}$ & $(\mathrm{T}-\mathrm{S})$ & $\mathrm{V}, \%$ \\
\hline $\begin{array}{l}\text { Wola Pszczó- } \\
\text { łecka A }\end{array}$ & $0-20$ & 2.92 & 0.5 & 0.033 & 0.026 & 0.079 & 0.638 & 3.558 & 2.92 & 17.93 \\
\hline $\begin{array}{l}\text { Wola Pszczó- } \\
\text { łecka A }\end{array}$ & $20-40$ & 1.54 & 0.1 & 0.013 & 0.009 & 0.018 & 0.140 & 1.680 & 1.54 & 8.333 \\
\hline $\begin{array}{l}\text { Wola Pszczó- } \\
\text { łecka A }\end{array}$ & $90-110$ & 1.53 & 0.1 & 0.018 & 0.009 & 0.031 & 0.158 & 1.688 & 1.53 & 9.360 \\
\hline
\end{tabular}

Source: own elaboration. 


\section{Characteristics of the flora and fungi - Wola Pszczółecka A}

Approximately $90 \%$ of the study plot is covered with plants. Fairly rich in species (22). Three species dominate: Hieracium pilosella (40\%), Conyza canadensis (15\%) and Rumex acetosa (15\%). The share of other species ranges from $5 \%$ to $0.5 \%$ of area coverage. They include: Achillea millefolium, Anthoxanthum aristatum, Convolvulus arvensis, Corynephorus canescens, Equisetum pratense, $\mathrm{Fe}$ stuca ovina viridis, Viola tricolor. Two species of moss were also identified: Bryum sp. and Polytrichum piliferum. Specimens of trees occur in the area - Pinus sylvesris (Tab. 6.1).

Fungi of the plot are represented by 13 species of macromycetes. The frequently found species include: Bovista plumbea, Macrolepiota procera, Lepiota alba, Rickenella fibula (Photo 7.2) occurs abundantly on mosses (Tab. 7.1).

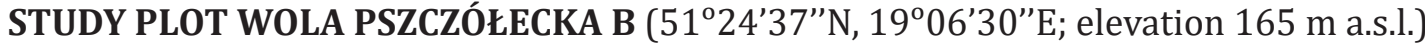
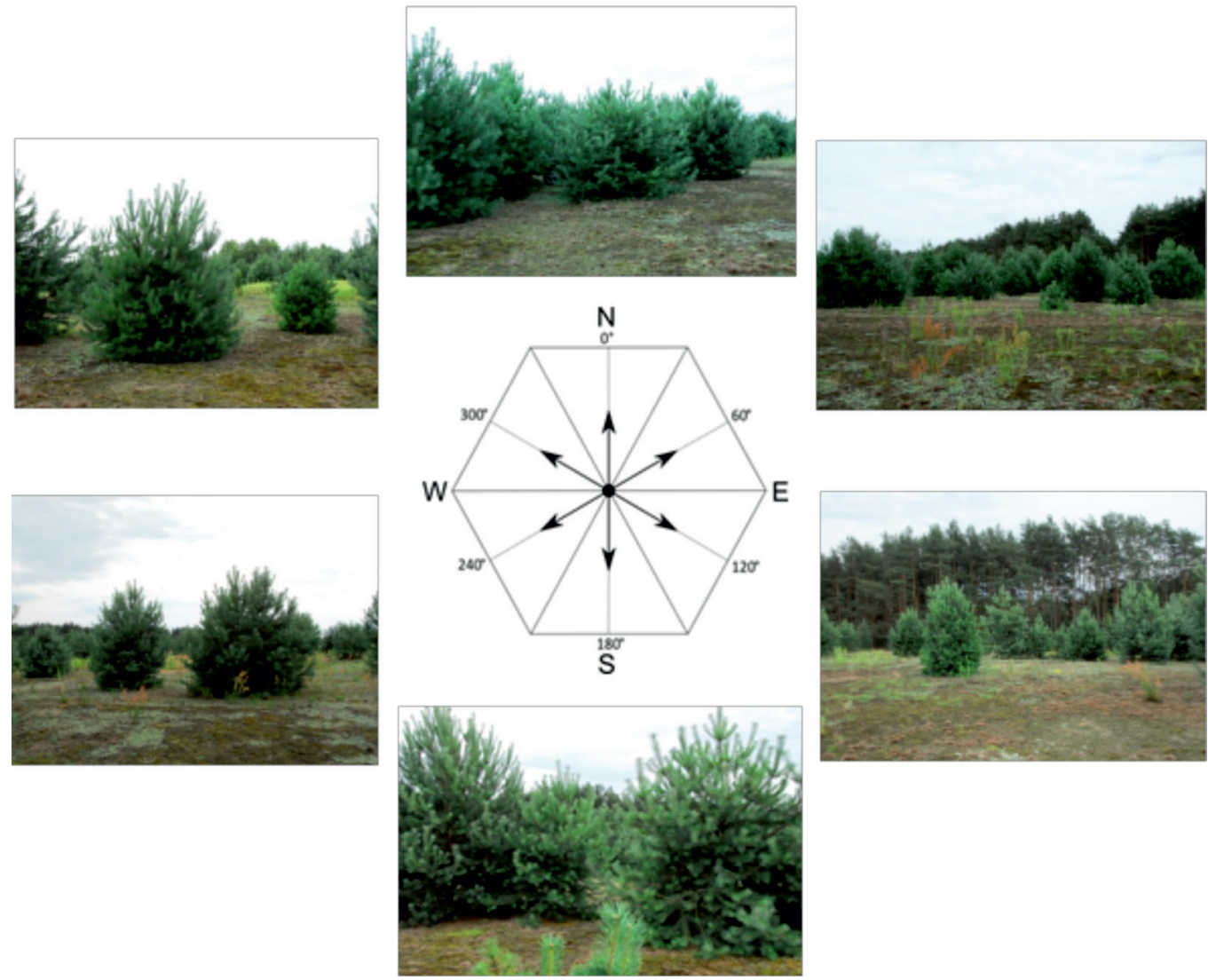

Fig. 5.33. Study plot Wola Pszczółecka B (photo E. Papińska, 2012)

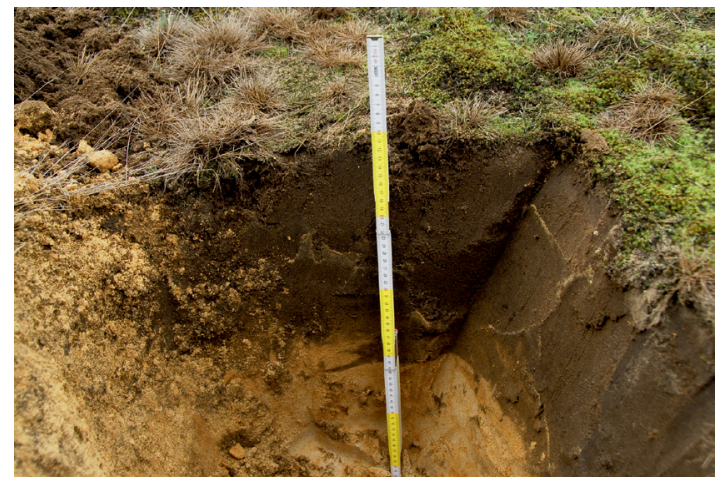

Depth Profile description

0-30 cm - humus, transformed, deepened by agricultural use (ploughing), coarse and medium sand, grey and dark brown

30-90 cm - horizon with dominating fine and medium sand

90-110 cm - horizon of stone pavement, turning into sand

Fig. 5.34. Soil pit in study plot Wola Pszczółecka B (photo. E. Papińska, 2012) 
Table 5.20. Study plot Wola Pszczółecka B. Granulometric and chemical properties of soil

\begin{tabular}{|c|c|c|c|c|c|c|c|c|c|c|c|c|}
\hline \multirow[b]{2}{*}{ Soil pit } & \multirow{2}{*}{$\begin{array}{l}\text { Sam- } \\
\text { pling } \\
\text { depth } \\
\text { cm }\end{array}$} & \multicolumn{9}{|c|}{$\begin{array}{c}\text { Grain size distribution } \\
\%\end{array}$} & \multirow{2}{*}{$\begin{array}{l}\text { Granu- } \\
\text { lome- } \\
\text { tric } \\
\text { sub- } \\
\text { group }\end{array}$} & \multirow{2}{*}{$\begin{array}{c}\text { Specific } \\
\text { surface } \\
\text { area } \\
\mathrm{m}^{2} \cdot \mathrm{g}^{-1}\end{array}$} \\
\hline & & $\begin{array}{c}2.0- \\
1.0 \\
\mathrm{~mm}\end{array}$ & $\begin{array}{c}1.0- \\
0.5 \\
\mathrm{~mm}\end{array}$ & $\begin{array}{l}0.5- \\
0.25 \\
\mathrm{~mm}\end{array}$ & $\begin{array}{c}0.25- \\
0.1 \\
\mathrm{~mm}\end{array}$ & $\begin{array}{l}0.1- \\
0.05 \\
\mathrm{~mm}\end{array}$ & $\begin{array}{c}0.05- \\
0.02 \\
\mathrm{~mm}\end{array}$ & $\begin{array}{c}0.02- \\
0.005 \\
\mathrm{~mm}\end{array}$ & $\begin{array}{c}0.005- \\
0.002 \\
\mathrm{~mm}\end{array}$ & $\begin{array}{c}<0.002 \\
\mathrm{~mm}\end{array}$ & & \\
\hline $\begin{array}{c}\text { Wola Pszczó- } \\
\text { łecka B }\end{array}$ & $0-20$ & 0 & 22.7 & 48.7 & 26.3 & 1.9 & 0.5 & 0 & 0 & 0 & $\mathrm{pl}$ & 0.0217 \\
\hline $\begin{array}{c}\text { Wola Pszczó- } \\
\text { łecka B }\end{array}$ & $20-40$ & 0 & 19.5 & 43.6 & 31.7 & 4.1 & 1.1 & 0 & 0 & 0 & $\mathrm{pl}$ & 0.0257 \\
\hline $\begin{array}{c}\text { Wola Pszczó- } \\
\text { łecka B }\end{array}$ & $90-100$ & 0 & 20.7 & 45.4 & 29.5 & 3.4 & 1.0 & 0 & 0 & 0 & $\mathrm{pl}$ & 0.0243 \\
\hline
\end{tabular}

\begin{tabular}{|c|c|c|c|c|c|c|c|c|c|c|}
\hline \multirow{2}{*}{ Soil pit } & \multirow{2}{*}{$\begin{array}{l}\text { Sam- } \\
\text { pling } \\
\text { depth } \\
\text { cm }\end{array}$} & \multirow{2}{*}{$\begin{array}{c}\text { Humus } \\
\%\end{array}$} & \multirow{2}{*}{$\begin{array}{c}\text { C total } \\
\%\end{array}$} & \multirow{2}{*}{$\begin{array}{c}\mathrm{N} \text { total } \\
\%\end{array}$} & \multirow{2}{*}{$\mathrm{C} / \mathrm{N}$} & \multicolumn{2}{|c|}{$\begin{array}{l}\mathrm{pH} \\
\text { in }\end{array}$} & \multicolumn{3}{|c|}{$\begin{array}{c}\text { Available nutrients } \\
\mathrm{mg} / 100 \mathrm{~g}\end{array}$} \\
\hline & & & & & & $\mathrm{KCl}$ & $\mathrm{H}_{2} \mathrm{O}$ & $\mathrm{P}_{2} \mathrm{O}_{5}$ & $\mathrm{~K}_{2} \mathrm{O}$ & $\mathrm{Mg}$ \\
\hline $\begin{array}{c}\text { Wola Pszczó- } \\
\text { łecka B }\end{array}$ & $0-20$ & 1.55 & 0.899 & 0.06 & 14.985 & 3.9 & 4.3 & 18.9 & 0.4 & 0.2 \\
\hline $\begin{array}{c}\text { Wola Pszczó- } \\
\text { łecka B }\end{array}$ & $20-40$ & - & - & - & - & 4.7 & 5.1 & 1.6 & 0.3 & 0.3 \\
\hline $\begin{array}{c}\text { Wola Pszczó- } \\
\text { łecka B }\end{array}$ & $90-100$ & - & - & - & - & 4.7 & 4.9 & 1.2 & 0.4 & 0.3 \\
\hline
\end{tabular}

\begin{tabular}{|c|c|c|c|c|c|c|c|c|c|c|}
\hline \multirow{2}{*}{ Soil pit } & \multirow{2}{*}{$\begin{array}{l}\text { Sam- } \\
\text { pling } \\
\text { depth } \\
\text { cm }\end{array}$} & \multirow{2}{*}{$\begin{array}{l}\text { Hydrolytic } \\
\text { acidity } \\
\mathrm{mmol} / 100 \mathrm{~g}\end{array}$} & \multicolumn{4}{|c|}{$\begin{array}{c}\text { Exchangeable cations } \\
\text { me } / 100 \mathrm{~g}\end{array}$} & \multicolumn{4}{|c|}{$\begin{array}{c}\text { Sorption capacity } \\
\text { me } / 100 \mathrm{~g}\end{array}$} \\
\hline & & & $\mathrm{Ca}^{2+}$ & $\mathrm{Mg}^{2+}$ & $\mathrm{Na}^{+}$ & $\mathrm{K}^{+}$ & $S$ & $\mathrm{~T}$ & $(\mathrm{~T}-\mathrm{S})$ & V, \% \\
\hline $\begin{array}{l}\text { Wola Pszczó- } \\
\text { łecka B }\end{array}$ & $0-20$ & 4.27 & 0.1 & 0.017 & 0.017 & 0.018 & 0.152 & 4.422 & 4.27 & 3.437 \\
\hline $\begin{array}{l}\text { Wola Pszczó- } \\
\text { łecka B }\end{array}$ & $20-40$ & 0.9 & 0.05 & 0.012 & 0.017 & 0.013 & 0.092 & 0.992 & 0.9 & 9.274 \\
\hline $\begin{array}{c}\text { Wola Pszczó- } \\
\text { tecka B }\end{array}$ & $90-100$ & 0.98 & 0.05 & 0.01 & 0.087 & 0.018 & 0.165 & 1.145 & 0.98 & 14.410 \\
\hline
\end{tabular}

Source: own elaboration.

\section{Characteristics of the flora and fungi - Wola Pszczółecka B}

Approximately $20 \%$ of the study plot is covered with plants and approximately $20 \%$ with lichens. Poor in species of plants (5). Three species dominate: Hieracium pilosella, Rumex acetosa and Pinus sylvesris, with each covering about $5 \%$ of the area. The other two species: Hypericum perforatum and Lupinus perennis exhibit a low coverage (about 5\%). Three species of lichens occur in the plot: Cladonia uncialis, Cladonia coccifera and Cladonia furcata (Tab. 6.1).

Fungi of the plot are sparse. They are represented by only 4 species of macromycetes, such as Bovista plumbea and B. nigrescens (Tab. 7.1). 


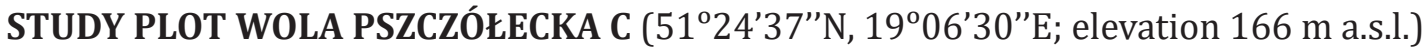
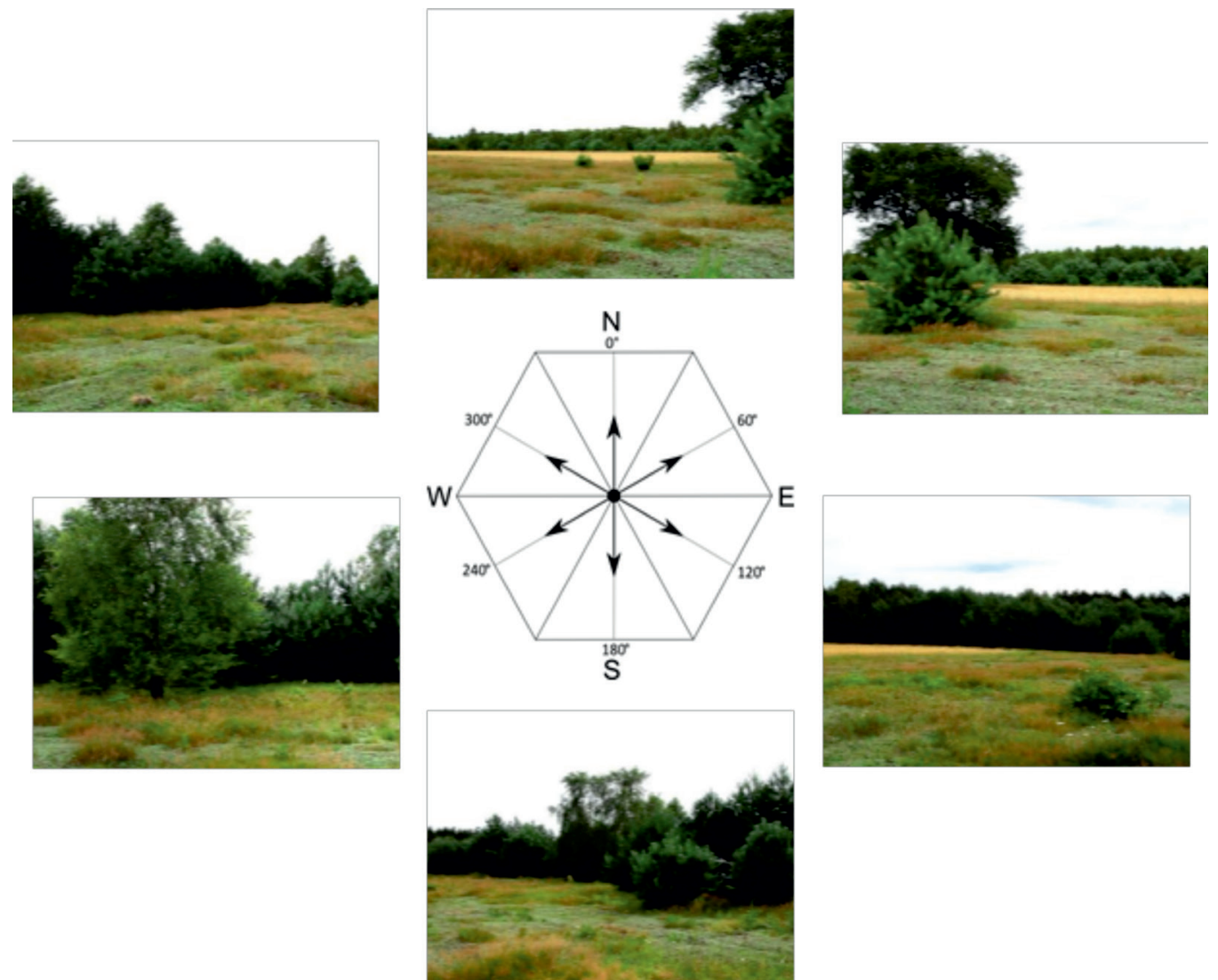

Fig. 5.35. Study plot Wola Pszczółecka C (photo E. Papińska, 2012)

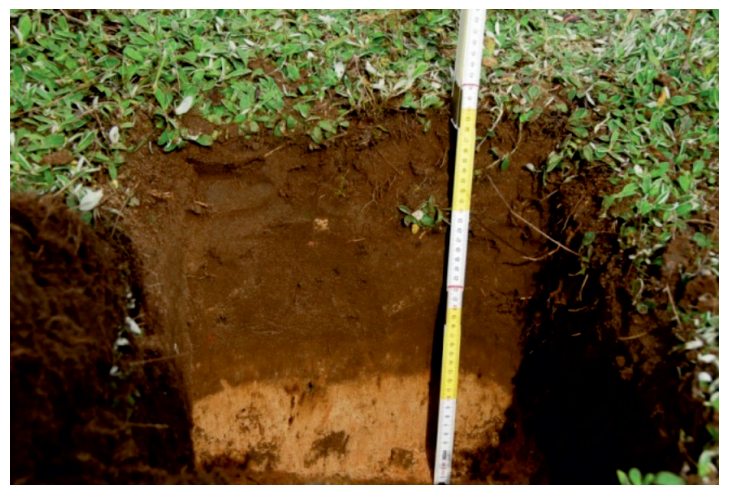

$$
\begin{aligned}
\text { Depth } & \text { Profile description } \\
1-0 \mathrm{~cm}- & \text { organic horizon } \\
0-30 \mathrm{~cm}- & \text { humus, transformed, deepened by } \\
& \text { agricultural use (ploughing), medium } \\
30-40 \mathrm{~cm}- & \text { sand, dark brown } \\
40-110 \mathrm{~cm}- & \text { loamy sand, beige sandy loam, beige }
\end{aligned}
$$

Fig. 5.36. Soil pit in study plot Wola Pszczółecka C (photo. E. Papińska, 2012) 
Table 5.21. Study plot Wola Pszczółecka C. Granulometric and chemical properties of soil

\begin{tabular}{|c|c|c|c|c|c|c|c|c|c|c|c|c|}
\hline \multirow[b]{2}{*}{ Soil pit } & \multirow{2}{*}{$\begin{array}{c}\text { Sam- } \\
\text { pling } \\
\text { depth } \\
\text { cm }\end{array}$} & \multicolumn{9}{|c|}{$\begin{array}{c}\text { Grain size distribution } \\
\%\end{array}$} & \multirow{2}{*}{$\begin{array}{c}\text { Granu- } \\
\text { lome- } \\
\text { tric } \\
\text { sub- } \\
\text { group }\end{array}$} & \multirow{2}{*}{$\begin{array}{c}\text { Specific } \\
\text { surface } \\
\text { area } \\
\mathrm{m}^{2} \cdot \mathrm{g}^{-1}\end{array}$} \\
\hline & & $\begin{array}{c}2.0- \\
1.0 \\
\mathrm{~mm}\end{array}$ & $\begin{array}{c}1.0- \\
0.5 \\
\mathrm{~mm}\end{array}$ & $\begin{array}{l}0.5- \\
0.25 \\
\mathrm{~mm}\end{array}$ & $\begin{array}{c}0.25- \\
0.1 \\
\mathrm{~mm}\end{array}$ & $\begin{array}{l}0.1- \\
0.05 \\
\mathrm{~mm}\end{array}$ & $\begin{array}{c}0.05- \\
0.02 \\
\mathrm{~mm}\end{array}$ & $\begin{array}{c}0.02- \\
0.005 \\
\mathrm{~mm}\end{array}$ & $\begin{array}{c}0.005- \\
0.002 \\
\mathrm{~mm}\end{array}$ & $\begin{array}{c}<0.002 \\
\mathrm{~mm}\end{array}$ & & \\
\hline $\begin{array}{c}\text { Wola Pszczó- } \\
\text { łecka C }\end{array}$ & $0-20$ & 1.2 & 23.4 & 35.1 & 20.3 & 5.4 & 5.3 & 5.8 & 2.1 & 1.3 & ps & 0.180 \\
\hline $\begin{array}{c}\text { Wola Pszczó- } \\
\text { łecka C }\end{array}$ & $20-40$ & 1.5 & 20.4 & 28.1 & 19.2 & 6.0 & 5.3 & 9.7 & 5.8 & 4.0 & pg & 0.441 \\
\hline $\begin{array}{c}\text { Wola Pszczó- } \\
\text { łecka C }\end{array}$ & $50-60$ & 0.1 & 13.3 & 24.0 & 17.3 & 5.9 & 7.8 & 15.0 & 9.6 & 7.0 & gp & 0.743 \\
\hline $\begin{array}{c}\text { Wola Pszczó- } \\
\text { łecka C }\end{array}$ & $\begin{array}{l}90- \\
100\end{array}$ & 0.3 & 14.6 & 24.3 & 16.7 & 5.8 & 7.2 & 14.6 & 9.6 & 7.0 & gp & 0.745 \\
\hline
\end{tabular}

\begin{tabular}{|c|c|c|c|c|c|c|c|c|c|c|}
\hline \multirow{2}{*}{ Soil pit } & \multirow{2}{*}{$\begin{array}{c}\text { Sam- } \\
\text { pling } \\
\text { depth } \\
\mathrm{cm}\end{array}$} & \multirow{2}{*}{$\begin{array}{c}\text { Humus } \\
\%\end{array}$} & \multirow{2}{*}{$\begin{array}{c}\text { C total } \\
\%\end{array}$} & \multirow{2}{*}{$\begin{array}{c}\mathrm{N} \text { total } \\
\%\end{array}$} & \multirow{2}{*}{$\mathrm{C} / \mathrm{N}$} & \multicolumn{2}{|c|}{$\begin{array}{l}\mathrm{pH} \\
\text { in }\end{array}$} & \multicolumn{3}{|c|}{$\begin{array}{l}\text { Available nutrients } \\
\mathrm{mg} / 100 \mathrm{~g}\end{array}$} \\
\hline & & & & & & $\mathrm{KCl}$ & $\mathrm{H}_{2} \mathrm{O}$ & $\mathrm{P}_{2} \mathrm{O}_{5}$ & $\mathrm{~K}_{2} \mathrm{O}$ & $\mathrm{Mg}$ \\
\hline $\begin{array}{c}\text { Wola Pszczó- } \\
\text { łecka C }\end{array}$ & $0-20$ & 1.42 & 0.824 & 0.072 & 11.440 & 3.9 & 4.4 & 6.4 & 1.5 & 0.4 \\
\hline $\begin{array}{c}\text { Wola Pszczó- } \\
\text { łecka C }\end{array}$ & $20-40$ & - & - & - & - & 4.1 & 4.9 & 1.2 & 1.5 & 0.9 \\
\hline $\begin{array}{c}\text { Wola Pszczó- } \\
\text { łecka C }\end{array}$ & $50-60$ & - & - & - & - & 3.9 & 5.2 & 0.9 & 3.3 & 6.5 \\
\hline $\begin{array}{l}\text { Wola Pszczó- } \\
\text { łecka C }\end{array}$ & $90-100$ & - & - & - & - & 4.6 & 6.3 & 0.9 & 3.2 & 8.0 \\
\hline
\end{tabular}

\begin{tabular}{|c|c|c|c|c|c|c|c|c|c|c|}
\hline \multirow{2}{*}{ Soil pit } & \multirow{2}{*}{$\begin{array}{l}\text { Sam- } \\
\text { pling } \\
\text { depth } \\
\text { cm } \\
\end{array}$} & \multirow{2}{*}{$\begin{array}{l}\text { Hydrolytic } \\
\text { acidity } \\
\text { mmol/100g }\end{array}$} & \multicolumn{4}{|c|}{$\begin{array}{c}\text { Exchangeable cations } \\
\text { me } / 100 \mathrm{~g}\end{array}$} & \multicolumn{4}{|c|}{$\begin{array}{c}\text { Sorption capacity } \\
\text { me } / 100 \mathrm{~g}\end{array}$} \\
\hline & & & $\mathrm{Ca}^{2+}$ & $\mathrm{Mg}^{2+}$ & $\mathrm{Na}^{+}$ & $\mathrm{K}^{+}$ & S & $\mathrm{T}$ & $(\mathrm{T}-\mathrm{S})$ & $\mathrm{V}, \%$ \\
\hline $\begin{array}{l}\text { Wola Pszczó- } \\
\text { łecka C }\end{array}$ & $0-20$ & 4.46 & 0.15 & 0.027 & 0.026 & 0.062 & 0.265 & 4.725 & 4.46 & 5.608 \\
\hline $\begin{array}{l}\text { Wola Pszczó- } \\
\text { łecka C }\end{array}$ & $20-40$ & 3.13 & 0.45 & 0.068 & 0.035 & 0.056 & 0.609 & 3.739 & 3.13 & 16.288 \\
\hline $\begin{array}{l}\text { Wola Pszczó- } \\
\text { łecka C }\end{array}$ & $50-60$ & 3.42 & 4.00 & 0.678 & 0.122 & 0.138 & 4.938 & 8.358 & 3.42 & 59.081 \\
\hline $\begin{array}{l}\text { Wola Pszczó- } \\
\text { łecka C }\end{array}$ & $90-100$ & 1.05 & 6.35 & 0.867 & 0.174 & 0.126 & 7.517 & 8.567 & 1.05 & 87.744 \\
\hline
\end{tabular}

Source: own elaboration.

\section{Characteristics of the flora and fungi - Wola Pszczółecka C}

Approximately $90 \%$ of the plot is covered with plants. Poor in species of plants (9). Two species dominate: Hieracium pilosella (65\%) and Agrostis capillaris $(15 \%)$. The other species do not cover more than $0.5 \%$ of the area each. They include: Achillea millefolium, Festuca, Jasione montana. There are some specimens of trees in the plot: $P i$ nus sylvestris and Betula pendula (Tab. 6.1).
Fungi of the plot are represented by 10 species of macromycetes. The most frequently found ones include: Marasmius oreades, Bovista plumbea. In the undergrowth, the following species were also found: Lepiota alba and Macrolepiota procera. Mycorrhizal species were represented by: Amanita muscaria, Boletus edulis, Inocybe asterospora and Suillus luteus (Tab. 7.1). 


\section{Analysis of granulometric composition and chemical properties of soils in the Wola Pszczółecka study plot group}

Data included in tables 5.19-5.21 indicate that the grain size distribution for two of three plots ( $A$ and $B$ ) is very similar, but it clearly differs for plot $\mathrm{C}$. The dominating fraction in all horizons of plots Wola Pszczółecka A and B is the sandy fraction $(95.4-100 \%)$, the share of silt is very small (the maximum of $4.5 \%$ ). The content of particles $<0.002 \mathrm{~mm}$ (clays) is insignificant and its maximum value is $0.3 \%$. In all horizons, the dominating fraction is medium sand, whose content ranges from 40.5 to $51.7 \%$. The share of coarse and fine sands is similar and ranges from 20.3 to $31.7 \%$ each. Thus, they are fractions of loose medium and coarse sand. Such a high content of the sandy fraction results in classifying the soils as the agronomic category of very light soils (I).

The granulometric composition at plot Wola Pszczółecka $C$ is different than that observed at sites $A$ and $B$ (hence the decision to take samples from an additional depth range of $50-60 \mathrm{~cm}$ ) and reflects the information given on the soil and agricultural map. Based on fraction analysis, the sample from the $0-20 \mathrm{~cm}$ depth was determined as the granulometric subgroup of slightly loamy sands (psg); then it turns into loamy sand (pg), to become sandy loam (gp) at the depth of 50-60 cm. The share of silts and clays clearly increases with profile depth, from $9.2 \%$ in the sample from $0-20 \mathrm{~cm}$ to more than $31 \%$ in samples taken below $50 \mathrm{~cm}$. The granulometric composition allows to qualify the soil as very light (I).

The value of specific surface area in samples from plots Wola Pszczółecka A and B is very low. It reaches $0.0195 \mathrm{~m}^{2} \cdot \mathrm{g}^{-1}$ in samples taken at the depth of 20-40 cm, where the share of the sandy fraction was $100 \%$. In other horizons, where some content of the silty and clayey fractions was present, the specific surface area value is higher, with the maximum value of $0.0617 \mathrm{~m}^{2} \cdot \mathrm{g}^{-1}$. The situation is different at plot $\mathrm{C}$, where the grain size distribution reveals a high content of silts and clays. The value of specific surface area ranges from $0.18 \mathrm{~m}^{2} \cdot \mathrm{g}^{-1}$ in the sample taken at the depth of $0-20 \mathrm{~cm}$ to $0.745 \mathrm{~m}^{2} \cdot \mathrm{g}^{-1}$ in the sample from the depth of $90-100 \mathrm{~cm}$.
The reaction in $\mathrm{KCl}$ in all horizons of the analysed plots is very acidic or acidic. The $\mathrm{pH}$ value in $\mathrm{H}_{2} \mathrm{O}$ at plot Wola Pszczółecka A decreases with depth, which is also reflected in a lower level of saturation with alkaline cations (V), which drops from nearly $18 \%$ in the sample from the $0-20 \mathrm{~cm}$ horizon to as little as 8-9\% in lower horizons. In samples from plot $\mathrm{B}$, the $\mathrm{pH}$ value is at its highest in the 20-40 cm horizon ( $\mathrm{pH} \mathrm{5.1)}$, and at plot $\mathrm{C}$, the value of this parameter increases with depth, from $\mathrm{pH} 4.4$ in the surface horizon to $\mathrm{pH} 6.3$ in the 90-100 cm horizon.

Low values of this parameter at plots A and B make the soils susceptible to fluctuation of the $\mathrm{pH}$ reaction and chemical degradation from acidic substances. Saturation with alkaline cations (V) in these soils is low and ranges from 3.4\% to nearly $18 \%$. Regrettably, the content of available nutrients in the case of potassium $\left(\mathrm{K}_{2} \mathrm{O}\right)$ and magnesium $(\mathrm{Mg})$ in all horizons of plots $\mathrm{A}$ and $\mathrm{B}$ is very low and low. At plot $C$, the level of saturation with alkaline cations increases noticeably, from 5.6\% (depth: $0-20 \mathrm{~cm}$ ) to nearly $88 \%$ (depth: $90-100 \mathrm{~cm}$ ), which means the base saturation is high. The fact is primarily influenced by the content of calcium cations $\left(\mathrm{Ca}^{2+}\right)$, which increases with depth. The content of magnesium reflects this rising trend with depth, from very low in the two upper horizons, through medium in the 50$60 \mathrm{~cm}$ horizon, to very high in the deepest sample. The content of phosphorus $\left(\mathrm{P}_{2} \mathrm{O}_{5}\right)$ in samples from all horizons of plots $A$ and $C$ is very low and low. Only at plot Wola Pszczółecka B, in the sample from the $0-20 \mathrm{~cm}$ horizon, was its high content recorded at $18.9 \%$.

The presence of humus compounds shapes the soil absorbing capacity. The content of organic carbon is high at all plots and ranges from $0.748 \%$ (A) to $0.899 \%$ (B). The content of humus is the lowest at plot Wola Pszczółecka A - 1.29\%, and the highest at plot Wola Pszczółecka B - 1.55\%, despite the fact that the vegetation occurring in the analysed area does not form a dense cover. 


\section{KRZĘTLE STUDY PLOT GROUP}

Krzetle study plot group lies at the elevation of 183-188 m a.s.l., in the Szczerców Basin (Kondracki 2002), about $1 \mathrm{~km}$ to the south-east of the border of the Warta-Widawka Interfluve Landscape Park, and about $10 \mathrm{~km}$ to the north-east of the border of the Załęcze Landscape Park. In administrative terms, the analysed group is located in Osjaków Commune, Wieluń District. The group is within a local kame hill. According to a geomorphological sketch by A. Dura-Gędek (2012), the kame hills border (mainly in the east) fluvioglacial plains, and to the west of them, there are undulating morainic plateaus. The 1:5000 soil and agricultural map of the area, shows the occurrence of leeched brown soils (Bw), of class 7 agricultural suitability (very poor rye), developed upon loose sands (pl). The soils were qualified as valuation class VI (http://geoportal.lodzkie. $\mathrm{pl} /$ imap/). Current usage - abandoned farmland. Characteristic scenery of the study plots is shown in photographs taken from the centre of the plot selected for the phytosociological inventory (Figures $5.37,5.39,5.41$ ). All the plots in the analysed group belong to a type 4 geocomplex. Also in this case, soil pits were excavated (Figures 5.38, $5.40,5.42$ ), from which samples were taken for laboratory studies, and their results were presented in tables 5.22-5.24.

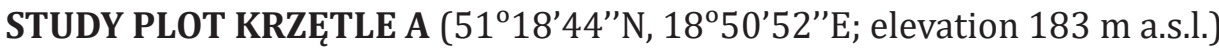

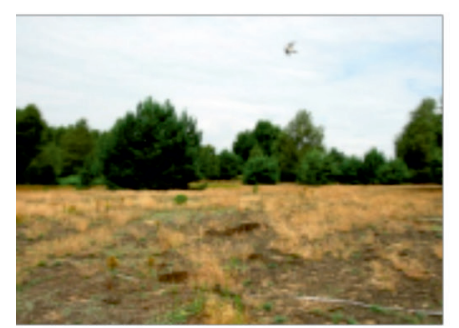

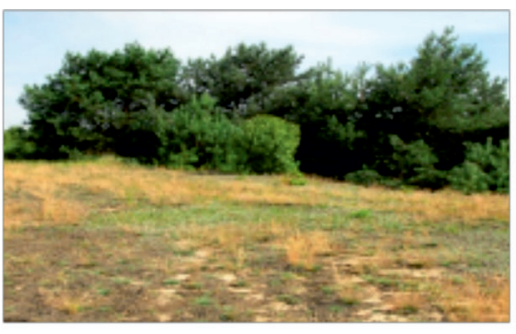
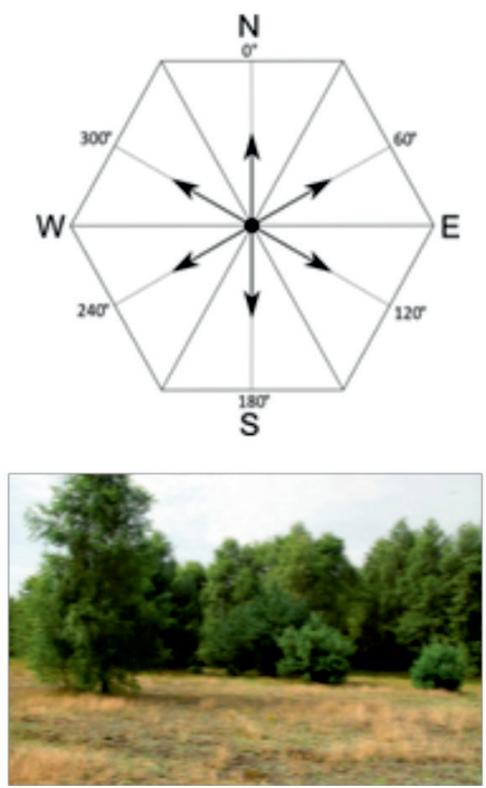
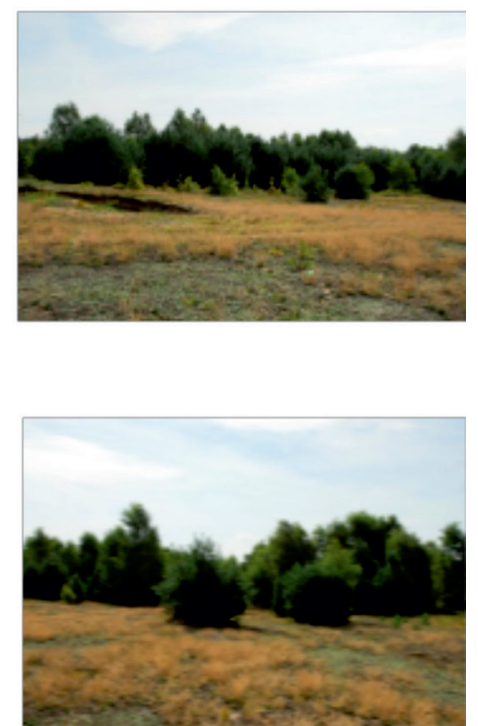

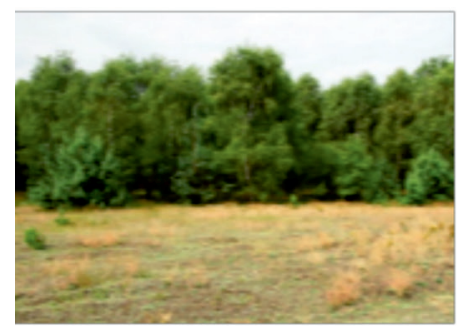

Fig. 5.37. Study plot Krzętle A (photo E. Papińska, 2012) 


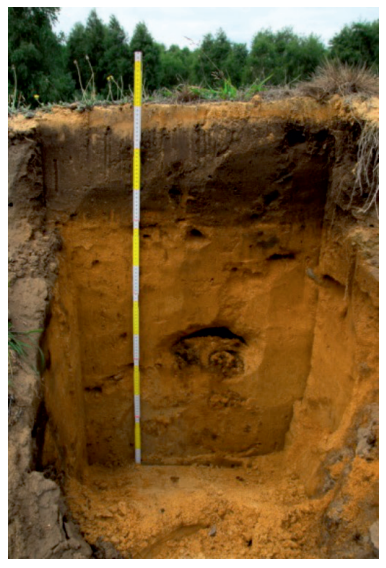

\author{
Depth Profile description \\ 1-0 cm - organic horizon \\ $0-5 \mathrm{~cm}$ - windrow, loose sand, rusty \\ 5-30 cm - humus, transformed, deepened by agricultural use \\ (ploughing), coarse and medium sand, grey and dark \\ brown \\ $30-90 \mathrm{~cm}$ - rusty horizon with dominating medium sand \\ 90-110 cm - horizon of parent rock, unsorted sand, rusty, with \\ cobbles and stones of $10-20 \mathrm{~cm}$ in diameter
}

Fig. 5.38. Soil pit in study plot Krzętle A (photo. E. Papińska, 2012)

Table 5.22. Study plot Krzętle A. Granulometric and chemical properties of soil

\begin{tabular}{|c|c|c|c|c|c|c|c|c|c|c|c|c|}
\hline \multirow[b]{2}{*}{ Soil pit } & \multirow{2}{*}{$\begin{array}{c}\text { Sampling } \\
\text { depth } \\
\mathrm{cm}\end{array}$} & \multicolumn{9}{|c|}{$\begin{array}{c}\text { Grain size distribution } \\
\%\end{array}$} & \multirow{2}{*}{$\begin{array}{l}\text { Granu- } \\
\text { lome- } \\
\text { tric } \\
\text { sub- } \\
\text { group }\end{array}$} & \multirow{2}{*}{$\begin{array}{c}\text { Spe- } \\
\text { cific } \\
\text { surface } \\
\text { area } \\
\mathrm{m}^{2} \cdot \mathrm{g}^{-1}\end{array}$} \\
\hline & & $\begin{array}{c}2.0- \\
1.0 \\
\mathrm{~mm}\end{array}$ & $\begin{array}{c}1.0- \\
0.5 \\
\mathrm{~mm}\end{array}$ & $\begin{array}{l}0.5- \\
0.25 \\
\mathrm{~mm}\end{array}$ & $\begin{array}{c}0.25- \\
0.1 \\
\mathrm{~mm}\end{array}$ & $\begin{array}{l}0.1- \\
0.05 \\
\mathrm{~mm}\end{array}$ & $\begin{array}{c}0.05- \\
0.02 \\
\mathrm{~mm}\end{array}$ & $\begin{array}{c}0.02- \\
0.005 \\
\mathrm{~mm}\end{array}$ & $\begin{array}{c}0.005- \\
0.002 \\
\mathrm{~mm}\end{array}$ & $\begin{array}{c}<0.002 \\
\mathrm{~mm}\end{array}$ & & \\
\hline Krzętle A & $0-20$ & 1.8 & 32.0 & 41.0 & 14.9 & 3.0 & 3.0 & 2.9 & 1.0 & 0.3 & pl śr & 0.0737 \\
\hline Krzętle A & $20-40$ & 2.7 & 37.9 & 41.7 & 11.7 & 1.7 & 1.2 & 1.9 & 0.9 & 0.3 & pl gr & 0.0617 \\
\hline Krzętle A & $90-110$ & 1.2 & 36.3 & 47.6 & 13.3 & 1.2 & 0.4 & 0.0 & 0.0 & 0.0 & pl gr & 0.0172 \\
\hline
\end{tabular}

\begin{tabular}{|c|c|c|c|c|c|c|c|c|c|c|}
\hline \multirow[t]{2}{*}{ Soil pit } & \multirow{2}{*}{$\begin{array}{c}\text { Sampling } \\
\text { depth } \\
\text { cm }\end{array}$} & \multirow{2}{*}{$\begin{array}{c}\text { Humus } \\
\%\end{array}$} & \multirow{2}{*}{$\begin{array}{l}\text { C total } \\
\%\end{array}$} & \multirow{2}{*}{$\begin{array}{l}\mathrm{N} \text { total } \\
\%\end{array}$} & \multirow[t]{2}{*}{$\mathrm{C} / \mathrm{N}$} & \multicolumn{2}{|c|}{$\begin{array}{l}\mathrm{pH} \\
\text { in }\end{array}$} & \multicolumn{3}{|c|}{$\begin{array}{l}\text { Available nutrients } \\
\mathrm{mg} / 100 \mathrm{~g}\end{array}$} \\
\hline & & & & & & $\mathrm{KCl}$ & $\mathrm{H}_{2} \mathrm{O}$ & $\mathrm{P}_{2} \mathrm{O}_{5}$ & $\mathrm{~K}_{2} \mathrm{O}$ & $\mathrm{Mg}$ \\
\hline Krzętle A & $0-20$ & 0.86 & 0.499 & 0.038 & 13.127 & 3.9 & 4.5 & 15.9 & 0.5 & 0.3 \\
\hline Krzętle A & $20-40$ & - & - & - & - & 4.6 & 5.0 & 4.8 & 0.3 & 0.3 \\
\hline Krzętle A & $90-110$ & - & - & - & - & 4.5 & 5.0 & 1.4 & 0.4 & 0.3 \\
\hline
\end{tabular}

\begin{tabular}{|c|c|c|c|c|c|c|c|c|c|c|}
\hline \multirow[t]{2}{*}{ Soil pit } & \multirow{2}{*}{$\begin{array}{c}\text { Sampling } \\
\text { depth } \\
\mathrm{cm}\end{array}$} & \multirow{2}{*}{$\begin{array}{l}\text { Hydrolytic } \\
\text { acidity } \\
\text { mmol/100g }\end{array}$} & \multicolumn{4}{|c|}{$\begin{array}{l}\text { Exchangeable cations } \\
\text { me } / 100 \mathrm{~g}\end{array}$} & \multicolumn{4}{|c|}{$\begin{array}{l}\text { Sorption capacity } \\
\text { me } / 100 \mathrm{~g}\end{array}$} \\
\hline & & & $\mathrm{Ca}^{2+}$ & $\mathrm{Mg}^{2+}$ & $\mathrm{Na}^{+}$ & $\mathrm{K}^{+}$ & S & $\mathrm{T}$ & (T-S) & $\mathrm{V}, \%$ \\
\hline Krzętle A & $0-20$ & 3.23 & 0.20 & 0.017 & 0.009 & 0.028 & 0.254 & 3.484 & 3.23 & 7.290 \\
\hline Krzętle A & $20-40$ & 1.40 & 0.15 & 0.013 & 0.004 & 0.013 & 0.180 & 1.580 & 1.40 & 11.392 \\
\hline Krzętle A & $90-110$ & 1.12 & 0.10 & 0.018 & 0.009 & 0.013 & 0.140 & 1.260 & 1.12 & 11.111 \\
\hline
\end{tabular}

Source: own elaboration.

\title{
Characteristics of the flora and fungi - Krzętle A
}

Approximately $80 \%$ of the plot is covered with plants. Poor in herbaceous plant species (10). Three species dominate among plants: Hieracium pilosella (about 30\%), Corynephorus canescens (about 20\%) (Photo 6.4) and Convolvulus arvensis (about 10\%). The share of other species does not exceed $1 \%$ of area coverage. The other identified species include: Anthoxantum aristatum, Equisetum pratense, Holcus mollis (Tab. 6.1). There are some saplings of Pinus sylvestris in the plot. A moss layer is also formed and covers about 5\% of the area. It consists of the following species: Brym sp., Ceratodon purpureus, Polytrichum piliferum. 
Fungi of the plot are represented by 12 species of macromycetes. The most frequently found species include: Marasmius oreades and Bovista plumbea. Less frequent species: Conocybe tenera,
Panaeolus fimicola. There are also a small number of sporocarps of mycorrhizal fungi in the plot: Amanita muscaria (Photo 7.3), Inocybe asterospora, Suillus bovinus and others (Tab. 7.1).

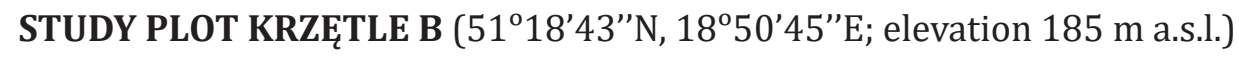
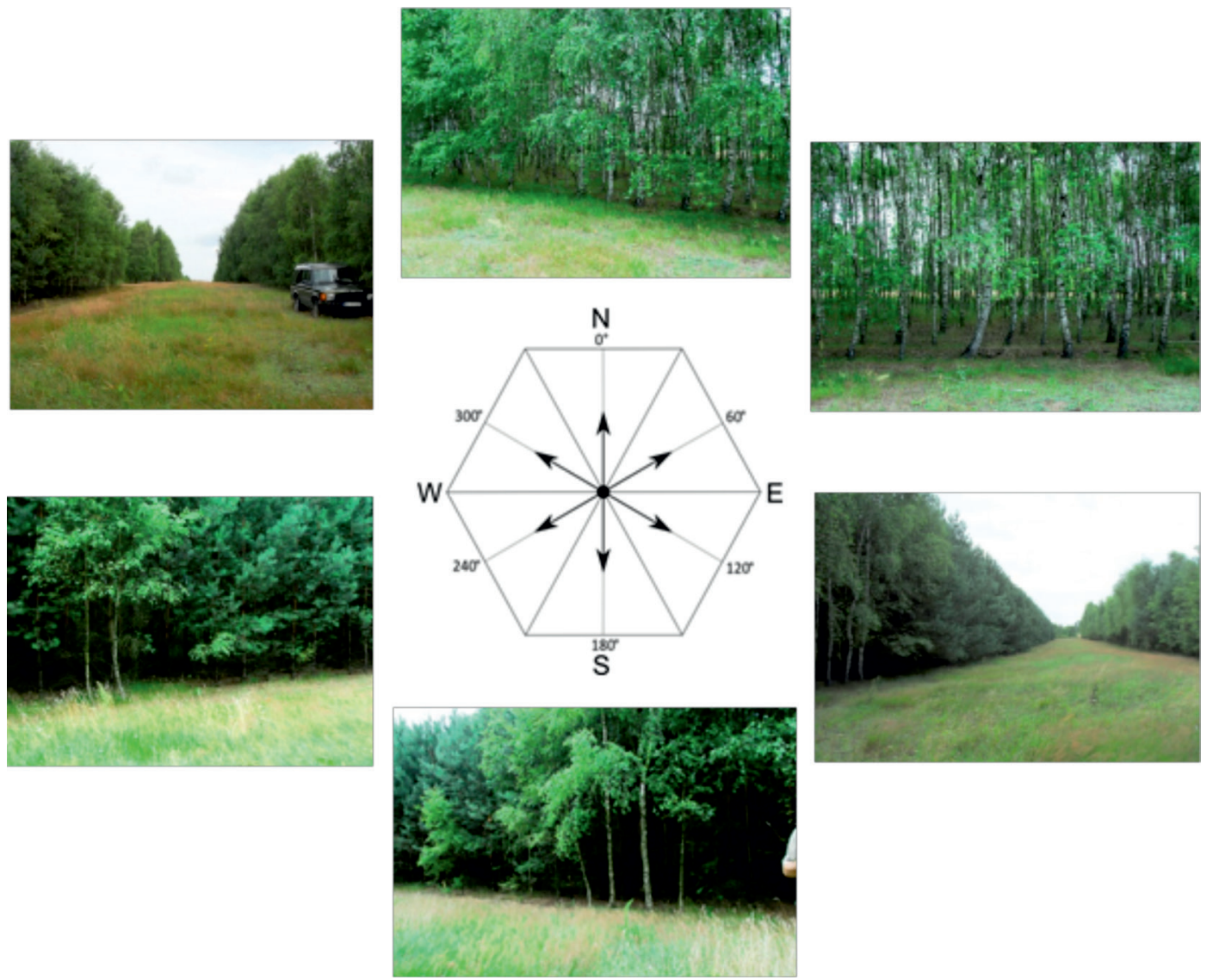

Fig. 5.39. Study plot Krzętle B (photo E. Papińska, 2012)

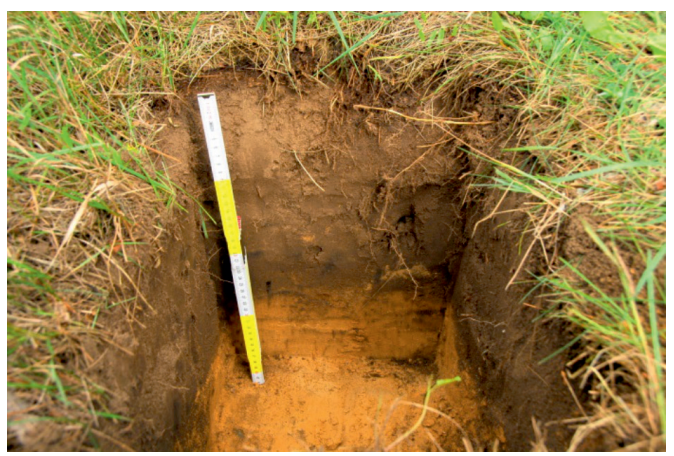

\author{
Depth Profile description \\ 1-0 cm - organic horizon \\ $0-30 \mathrm{~cm}$ - humus, grey and dark brown medium sand with \\ local material (flint) \\ $30-90 \mathrm{~cm}$ - rusty horizon with dominating medium sand \\ with loamy inclusions \\ 90-110 cm - horizon of parent rock, fine sand
}

Fig. 5.40. Soil pit in study plot Krzętle B (photo. E. Papińska, 2012) 
Table 5.23. Study plot Krzętle B. Granulometric and chemical properties of soil

\begin{tabular}{|c|c|c|c|c|c|c|c|c|c|c|c|c|}
\hline \multirow[b]{2}{*}{ Soil pit } & \multirow{2}{*}{$\begin{array}{c}\text { Sampling } \\
\text { depth } \\
\mathrm{cm}\end{array}$} & \multicolumn{9}{|c|}{$\begin{array}{c}\text { Grain size distribution } \\
\%\end{array}$} & \multirow{2}{*}{$\begin{array}{l}\text { Granu- } \\
\text { lome- } \\
\text { tric } \\
\text { sub- } \\
\text { group }\end{array}$} & \multirow{2}{*}{$\begin{array}{c}\text { Specific } \\
\text { surface } \\
\text { area } \\
\mathrm{m}^{2} \cdot \mathrm{g}-1\end{array}$} \\
\hline & & $\begin{array}{c}2.0- \\
1.0 \\
\mathrm{~mm}\end{array}$ & $\begin{array}{c}1.0- \\
0.5 \\
\mathrm{~mm}\end{array}$ & $\begin{array}{l}0.5- \\
0.25 \\
\mathrm{~mm}\end{array}$ & $\begin{array}{c}0.25- \\
0.1 \\
\mathrm{~mm}\end{array}$ & $\begin{array}{l}0.1- \\
0.05 \\
\mathrm{~mm}\end{array}$ & $\begin{array}{c}0.05- \\
0.02 \\
\mathrm{~mm}\end{array}$ & $\begin{array}{c}0.02- \\
0.005 \\
\mathrm{~mm}\end{array}$ & $\begin{array}{c}0.005- \\
0.002 \\
\mathrm{~mm}\end{array}$ & $\begin{array}{c}<0.002 \\
\mathrm{~mm}\end{array}$ & & \\
\hline Krzętle B & $0-20$ & 2.2 & 29.1 & 38.2 & 17.2 & 4.7 & 4.2 & 3.2 & 0.9 & 0.4 & $\mathrm{pl}$ & 0.0807 \\
\hline Krzętle B & $20-40$ & 1.3 & 28.8 & 42.6 & 16.9 & 2.0 & 2.7 & 3.0 & 1.6 & 1.0 & $\mathrm{pl}$ & 0.1270 \\
\hline Krzętle B & $90-110$ & 0.4 & 27.3 & 46.6 & 18.6 & 1.0 & 1.3 & 2.5 & 1.7 & 0.6 & $\mathrm{pl}$ & 0.0975 \\
\hline
\end{tabular}

\begin{tabular}{|c|c|c|c|c|c|c|c|c|c|c|}
\hline \multirow[t]{2}{*}{ Soil pit } & \multirow{2}{*}{$\begin{array}{c}\text { Sampling } \\
\text { depth } \\
\mathrm{cm}\end{array}$} & \multirow{2}{*}{$\begin{array}{c}\text { Humus } \\
\%\end{array}$} & \multirow{2}{*}{$\begin{array}{c}\text { C total } \\
\%\end{array}$} & \multirow{2}{*}{$\begin{array}{c}\mathrm{N} \text { total } \\
\%\end{array}$} & \multirow[t]{2}{*}{$\mathrm{C} / \mathrm{N}$} & \multicolumn{2}{|c|}{$\begin{array}{l}\mathrm{pH} \\
\text { in }\end{array}$} & \multicolumn{3}{|c|}{$\begin{array}{l}\text { Available nutrients } \\
\mathrm{mg} / 100 \mathrm{~g}\end{array}$} \\
\hline & & & & & & $\mathrm{KCl}$ & $\mathrm{H}_{2} \mathrm{O}$ & $\mathrm{P}_{2} \mathrm{O}_{5}$ & $\mathrm{~K}_{2} \mathrm{O}$ & $\mathrm{Mg}$ \\
\hline Krzętle B & $0-20$ & 1.3 & 0.754 & 0.06 & 12.568 & 4.1 & 5.0 & 10.8 & 2.7 & 0.8 \\
\hline Krzętle B & $20-40$ & - & - & - & - & 4.3 & 5.1 & 2.1 & 1.6 & 0.8 \\
\hline Krzętle B & $90-110$ & - & - & - & - & 4.4 & 5.5 & 1.0 & 3.8 & 2.4 \\
\hline
\end{tabular}

\begin{tabular}{|c|c|c|c|c|c|c|c|c|c|c|}
\hline & \multirow{2}{*}{ Soil pit } & \multirow{2}{*}{$\begin{array}{c}\text { Sempling } \\
\text { depth } \\
\mathrm{cm}\end{array}$} & $\begin{array}{c}\text { Hydrolytic } \\
\text { acidity } \\
\text { mmol/100g }\end{array}$ & \multicolumn{4}{|c|}{$\begin{array}{c}\text { Exchangeable cations } \\
\mathrm{me} / 100 \mathrm{~g}\end{array}$} & \multicolumn{4}{c|}{$\begin{array}{c}\text { Sorption capacity } \\
\text { me/100g }\end{array}$} \\
\cline { 5 - 14 } & & $\mathrm{Ca}^{2+}$ & $\mathrm{Mg}^{2+}$ & $\mathrm{Na}^{+}$ & $\mathrm{K}^{+}$ & $\mathrm{S}$ & $\mathrm{T}$ & $(\mathrm{T}-\mathrm{S})$ & $\mathrm{V}, \%$ \\
\hline Krzętle B & $0-20$ & 3.37 & 0.45 & 0.070 & 0.017 & 0.092 & 0.629 & 3.999 & 3.37 & 15.729 \\
\hline Krzętle B & $20-40$ & 1.82 & 0.30 & 0.067 & 0.026 & 0.049 & 0.442 & 2.262 & 1.82 & 19.540 \\
\hline Krzętle B & $90-110$ & 0.98 & 0.50 & 0.210 & 0.017 & 0.072 & 0.799 & 1.779 & 0.98 & 44.913 \\
\hline
\end{tabular}

Source: own elaboration.

\section{Characteristics of the flora and fungi - Krzętle B}

Approximately $90 \%$ of the study plot is covered with plants. Not very rich in plant species (15). One species dominates: Agrostis capillaris (about $50 \%$ ). The share of other species ranges from $5 \%$ to $0.5 \%$. The identified species include: Achillea millefolium, Apera spica-venti, Convolvulus arvensis, Conyza canadensis, Festuca ovina (Tab. 6.1). There are also several saplings of trees: Quercus robur. A moss layer is also formed and it covers about $25 \%$ of the plot. It consists of two species: Ceratodon purpurea and Pleurozium schreberii.
Fungi of the plot are abundant. It is represented by 14 species of macromycetes. The most frequently found species is Marasmius oreades. Numerous sporocarps of mycorrhizal fungi were also found in the area, such as: Scleroderma citrina, Amanita muscaria, Suillus bovinus. Numerous sporocarps of Trichaptum abietinum were identified on pine branches (Tab. 7.1). 
STUDY PLOT KRZĘTLE C (51 ${ }^{\circ} 19^{\prime} 41^{\prime \prime} \mathrm{N}, 18^{\circ} 50^{\prime} 37^{\prime \prime}$ E; elevation 186 m a.s.l.)
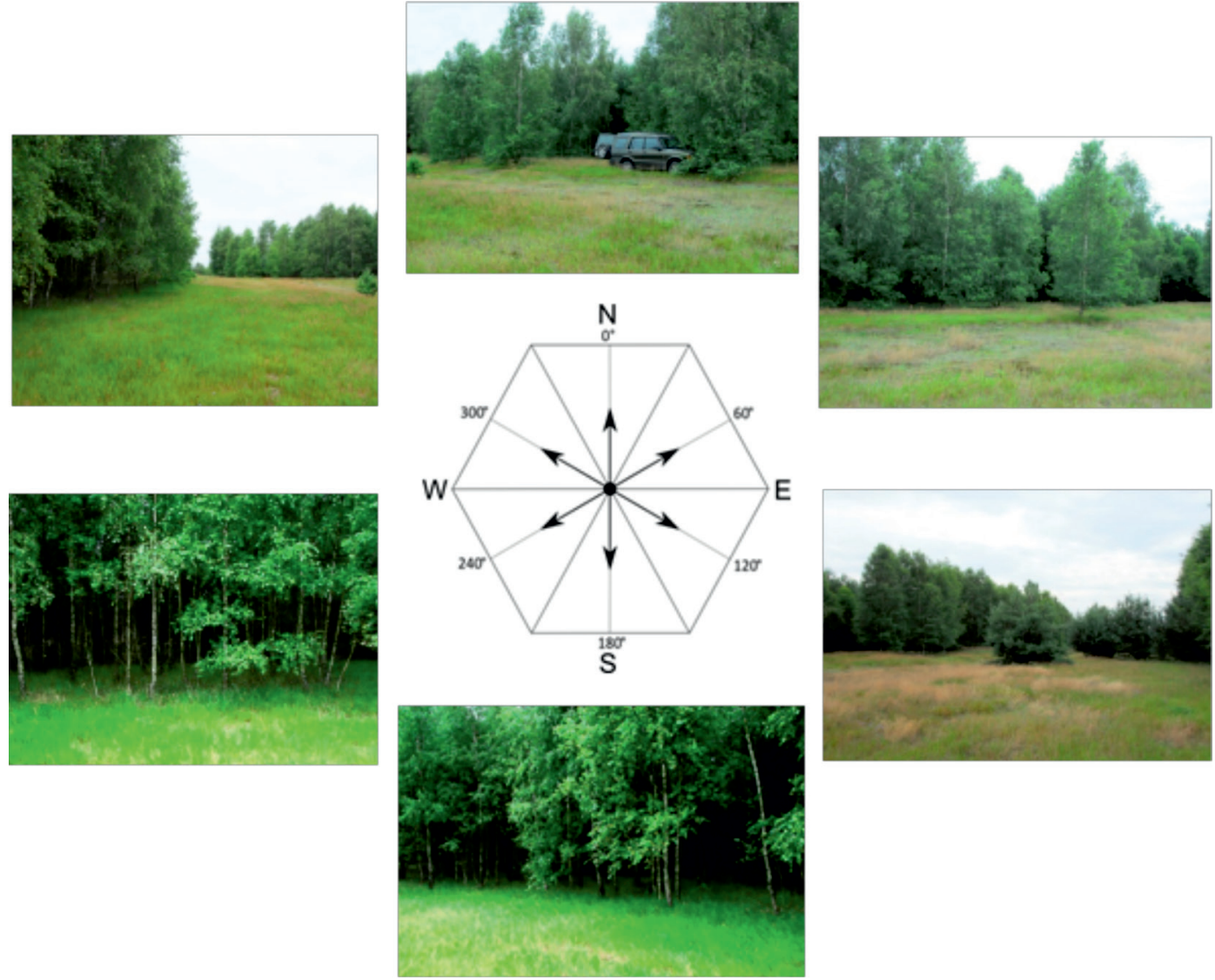

Fig. 5.41. Study plot Krzętle B (photo E. Papińska, 2012)

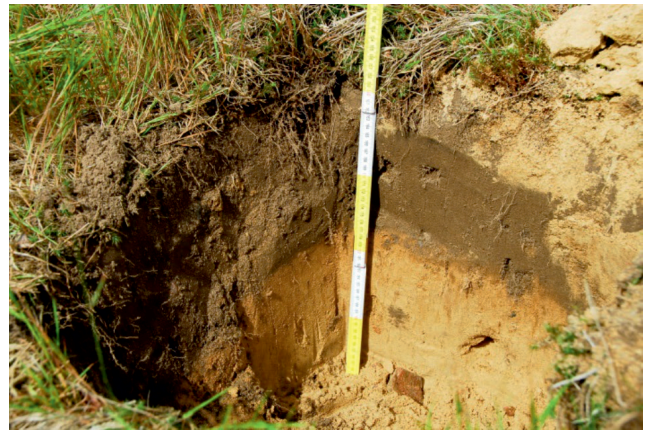

Depth Profile description

1-0 cm - organic horizon

0-20 cm - humus, grey and dark brown medium sand with local material (flint)

20-40 cm - rusty horizon with dominating medium sand 40-110 cm - horizon of parent rock, medium sand, light yellow

Fig. 5.42. Soil pit in study plot Krzętle C (photo. E. Papińska, 2012) 
Table 5.24. Krzętle C. Granulometric and chemical properties of soil

\begin{tabular}{|c|c|c|c|c|c|c|c|c|c|c|c|c|}
\hline \multirow{2}{*}{$\begin{array}{c}\text { Sampling } \\
\text { depth } \\
\mathrm{cm}\end{array}$} & \multirow[b]{2}{*}{ Soil pit } & \multicolumn{9}{|c|}{$\begin{array}{c}\text { Grain size distribution } \\
\%\end{array}$} & \multirow{2}{*}{$\begin{array}{l}\text { Granu- } \\
\text { lome- } \\
\text { tric } \\
\text { sub- } \\
\text { group }\end{array}$} & \multirow{2}{*}{$\begin{array}{c}\text { Spe- } \\
\text { cific } \\
\text { surface } \\
\text { area } \\
\mathrm{m}^{2} \cdot \mathrm{g}^{-1}\end{array}$} \\
\hline & & $\begin{array}{l}2.0- \\
1.0 \\
\mathrm{~mm}\end{array}$ & $\begin{array}{l}1.0- \\
0.5 \\
\mathrm{~mm}\end{array}$ & $\begin{array}{l}0.5- \\
0.25 \\
\mathrm{~mm}\end{array}$ & $\begin{array}{c}0.25- \\
0.1 \\
\mathrm{~mm}\end{array}$ & $\begin{array}{l}0.1- \\
0.05 \\
\mathrm{~mm}\end{array}$ & $\begin{array}{l}0.05- \\
0.02 \\
\mathrm{~mm}\end{array}$ & $\begin{array}{c}0.02- \\
0.005 \\
\mathrm{~mm}\end{array}$ & $\begin{array}{c}0.005- \\
0.002 \\
\mathrm{~mm}\end{array}$ & $\begin{array}{c}<0.002 \\
\mathrm{~mm}\end{array}$ & & \\
\hline Krzętle C & $0-20$ & 1.3 & 23.1 & 33.2 & 22.3 & 7.9 & 5.1 & 4.4 & 1.6 & 1.0 & $\mathrm{pl}$ & 0.1470 \\
\hline Krzętle C & $20-40$ & 0.4 & 27.9 & 46.1 & 19.9 & 2.6 & 1.1 & 1.2 & 0.6 & 0.1 & $\mathrm{pl}$ & 0.0442 \\
\hline Krzętle C & $90-110$ & 0.0 & 31.1 & 54.2 & 10.8 & 0.0 & 0.0 & 2.0 & 1.3 & 0.4 & $\mathrm{pl}$ & 0.0716 \\
\hline
\end{tabular}

\begin{tabular}{|c|c|c|c|c|c|c|c|c|c|c|}
\hline \multirow[t]{2}{*}{ Soil pit } & \multirow{2}{*}{$\begin{array}{l}\text { Sampling } \\
\text { depth } \\
\mathrm{cm}\end{array}$} & \multirow{2}{*}{$\begin{array}{c}\text { Humus } \\
\%\end{array}$} & \multirow{2}{*}{$\begin{array}{c}\mathrm{C} \text { total } \\
\%\end{array}$} & \multirow{2}{*}{$\begin{array}{c}\mathrm{N} \text { total } \\
\%\end{array}$} & \multirow[t]{2}{*}{$\mathrm{C} / \mathrm{N}$} & \multicolumn{2}{|c|}{$\begin{array}{l}\mathrm{pH} \\
\text { in }\end{array}$} & \multicolumn{3}{|c|}{$\begin{array}{c}\text { Available nutrients } \\
\mathrm{mg} / 100 \mathrm{~g}\end{array}$} \\
\hline & & & & & & $\mathrm{KCl}$ & $\mathrm{H}_{2} \mathrm{O}$ & $\mathrm{P}_{2} \mathrm{O}_{5}$ & $\mathrm{~K}_{2} \mathrm{O}$ & $\mathrm{Mg}$ \\
\hline Krzętle C & $0-20$ & 1.22 & 0.708 & 0.06 & 11.794 & 3.8 & 4.4 & 14.2 & 1.1 & 0.3 \\
\hline Krzętle C & $20-40$ & - & - & - & - & 4.5 & 4.9 & 5.3 & 0.3 & 0.2 \\
\hline Krzętle C & $90-110$ & - & - & - & - & 4.3 & 5.4 & 2.4 & 2.1 & 1.3 \\
\hline
\end{tabular}

\begin{tabular}{|c|c|c|c|c|c|c|c|c|c|c|}
\hline \multirow[t]{2}{*}{ Soil pit } & \multirow{2}{*}{$\begin{array}{l}\text { Sampling } \\
\text { depth } \\
\mathrm{cm}\end{array}$} & \multirow{2}{*}{$\begin{array}{l}\text { Hydrolytic } \\
\text { acidity } \\
\text { mmol/100g }\end{array}$} & \multicolumn{4}{|c|}{$\begin{array}{c}\text { Exchangeable cations } \\
\text { me } / 100 \mathrm{~g}\end{array}$} & \multicolumn{4}{|c|}{$\begin{array}{c}\text { Sorption capacity } \\
\text { me } / 100 \mathrm{~g}\end{array}$} \\
\hline & & & $\mathrm{Ca}^{2+}$ & $\mathrm{Mg}^{2+}$ & $\mathrm{Na}^{+}$ & $\mathrm{K}^{+}$ & S & $\mathrm{T}$ & $(\mathrm{T}-\mathrm{S})$ & $\mathrm{V}, \%$ \\
\hline Krzętle C & $0-20$ & 3.94 & 0.10 & 0.020 & 0.009 & 0.049 & 0.178 & 4.118 & 3.94 & 4.322 \\
\hline Krzętle C & $20-40$ & 1.22 & 0.10 & 0.015 & 0.009 & 0.013 & 0.137 & 1.357 & 1.22 & 10.096 \\
\hline Krzętle C & $90-110$ & 1.03 & 0.45 & 0.098 & 0.017 & 0.056 & 0.621 & 1.651 & 1.03 & 37.614 \\
\hline
\end{tabular}

Source: own elaboration.

\section{Characteristics of the flora and fungi - Krzętle C}

Approximately $100 \%$ of the study plot is covered with plants. Poor in vascular plants (8). The flora is dominated by one species: Agrostis capillaris (about 60\%). Other species with a significant share include Holcus mollis (about 20\%), Hieracium pilosella (about 10\%). The share of other species ranges from $5 \%$ to $0.5 \%$. They include: Anthoxanthum odoratum, Corynephorus canescens (Tab. 6.1). The plot also features a few saplings of Quercus robur. The moss layer is formed scantily and is represented by one species: Polytrichum piliferum.
Fungi of the plot are sparse. They are represented by 8 species of macromycetes. The frequently found species is Crinipellis scabella, occurring on grasses, mainly on Agrostis capillaris. Among land fungi, the abundantly fruiting species were: Bovista pumbea and Calvatias: Calvatia utriformis and C. excipuliformis. There were a small number of sporocarps of the mycorrhizal Amanita muscaria (Tab. 7.1).

\section{Analysis of granulometric composition and chemical properties of soils in the Krzętle study plot group}

The results of granulometric analysis presented in tables 5.22-5.24, indicate the domination of the sandy fraction at all plots and in all horizons from which samples were taken (Figures 5.38,
5.40 and 5.42). The share of this fraction ranges from $87.8 \%$ to $99.6 \%$ in the sample from the depth of $90-110 \mathrm{~cm}$. The share of the silty fraction exceeds $10 \%$ in just one sample (Krzętle C, 
horizon 0-20 cm). Its lowest content was found at plot Krzętle A - 0.4\% - at the depth of 90-100 cm. The clayey fraction is insignificant and amounts to a maximum of $1 \%$ in samples from the depth of $0-20 \mathrm{~cm}$ (plot C) and 20-40 cm (plot B). The high content of material in granulometric groups of coarse $(20-32 \%)$ and medium sands $(40 \%$ and above) is worth noticing. The presented grain size distribution of the analysed soils provides a basis for classifying them as very light soils (I) in the agronomic category.

A derivative of grain size distribution is the value of specific surface area. It is the highest for the sample taken from the smallest depth at plot Krzętle C $-0.147 \mathrm{~m}^{2} \cdot \mathrm{g}^{-1}$. This is due to the highest recorded share of silts (11.1\%) and clays (1\%) of all the profile. Also at this plot, the lowest value of this parameter of $0.0172 \mathrm{~m}^{2} \cdot \mathrm{g}^{-1}$ was recorded at the depth of 20-40 cm.

Analysis of $\mathrm{pH}$ reaction indicates high soil acidification, the $\mathrm{pH}$ value in $\mathrm{KCl}$ for the surface horizon at plot Krzętle C was as low as 3.8 (very acidic). Only one sample from the depth of 20-40 cm at plot Krzętle A reveals a slight increase of the $\mathrm{pH}$ value to 4.6 (acidic). In all other sam- ples at the analysed group, the pH value was very acidic. It influences the saturation degree with alkaline cations (V), which is only $7.29 \%$ at plot Krzętle $\mathrm{A}$ in the near-surface horizon and gradually increases to more than $11 \%$ in the remaining samples. At plot Krzętle B, the parameter changes with depth from $15.729 \%(0-20 \mathrm{~cm})$ to $44.913 \%$ $(90-100 \mathrm{~cm})$, and at plot Krzętle C from 4.322\% $(0-20 \mathrm{~cm})$ to $37.614 \%(90-100 \mathrm{~cm})$. The assessment of plant nutrient availability indicates very low availability of magnesium $(\mathrm{Mg})$ and potassium $\left(\mathrm{K}_{2} \mathrm{O}\right)$ in all samples from plot Krzętle A. At plots $\mathrm{B}$ and $\mathrm{C}$ both parameters are within very low and low availability classes, except for the sample taken from the depth of $90-100 \mathrm{~cm}$ at plot Krzętle B, which is found in the medium magnesium availability class. The content of phosphorus $\left(\mathrm{P}_{2} \mathrm{O}_{5}\right)$ indicates a higher diversity, from high availability (Krzętle A, $0-20 \mathrm{~cm}$ ), through medium (Krzętle $B$ and C, $0-20 \mathrm{~cm}$ ), to low and very low in the remaining cases.

Similarly to other analysed study plot groups, the content of humus was determined in the $0-20 \mathrm{~cm}$ horizon, and it ranges from $0.86 \%$ at plot Krzętle A to $1.3 \%$ at plot Krzętle B.

\section{RACISZYN STUDY PLOT GROUP}

The Raciszyn study plot group is located in Działoszyn Commune, Pajęczno District. It lies at the elevation of 210-220 m a.s.l., in the Wielun Upland (Kondracki 2002), about 800-1 $200 \mathrm{~m}$ to the east of the border of the Załecze Landscape Park. In geomorphological terms, it is a fluvioglacial plain, formed of fluvioglacial sands of the Odra Glaciation (Haisig, Wilanowski 2000). In the close vicinity, there are some outcrops of Upper Jurassic limestone, which were excavated in numerous quarries. They form some clearly dominating surfaces in the landscape - island hills. At numerous spots, the Mesozoic substratum lies close to the land surface, covered with a thin layer of fluvioglacial deposits (Laskowski, Papińska, Tołoczko 2001; Papińska 2001a, 2001b; Papińska, Tołoczko 2002). At plots Raciszyn A and
B, the 1:5000 soil and agricultural map shows leeched brown soils (Bw), belonging to class 6 of agricultural suitability (poor rye). They were formed on slightly loamy sands (psg) lying upon limestone $(\mathrm{w})$. The soils were qualified as valuation class V (http://geoportal.lodzkie.pl/ imap/). At plot Raciszyn C, the leeched brown soils were classified as agricultural suitability class 7 (very poor rye) and valuation class VI, and they are formed on loose sands (pl). The study plot group is located in a type 6 geocomplex, and its characteristics are presented in photographs (Figures 5.43, 5.45, 5.47). Soil pits were excavated in order to document the plots (Figures 5.44, 5.46, 5.48), from which samples were taken for laboratory analyses, and their results were presented in tables 5.25-5.27. 
STUDY PLOT RACISZYN A (51 $05^{\prime} 26^{\prime \prime} \mathrm{N}, 18^{\circ} 51^{\prime} 13^{\prime \prime}$; elevation 220 m a.s.l.)
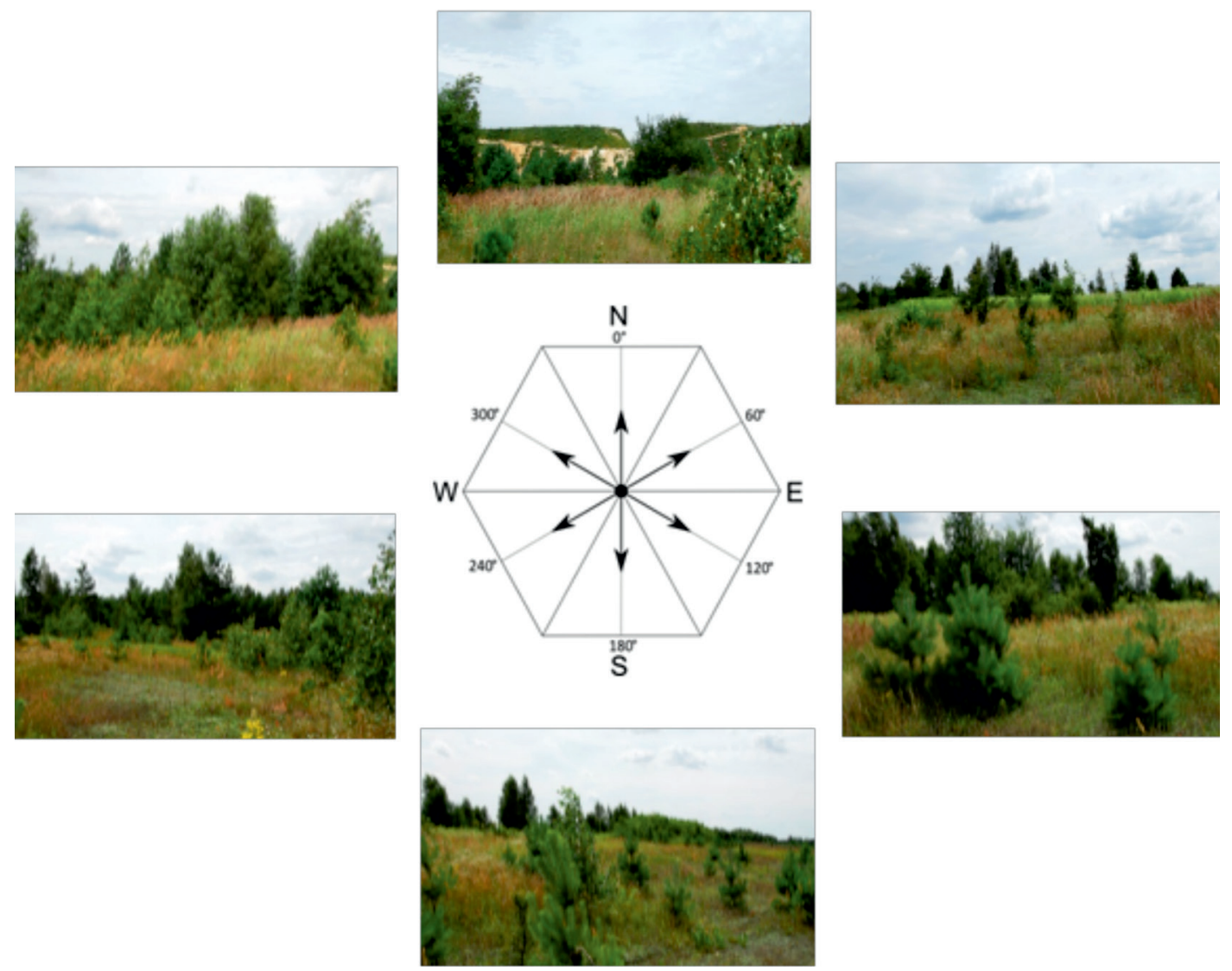

Fig. 5.43. Study plot Raciszyn A (photo E. Papińska, 2012)

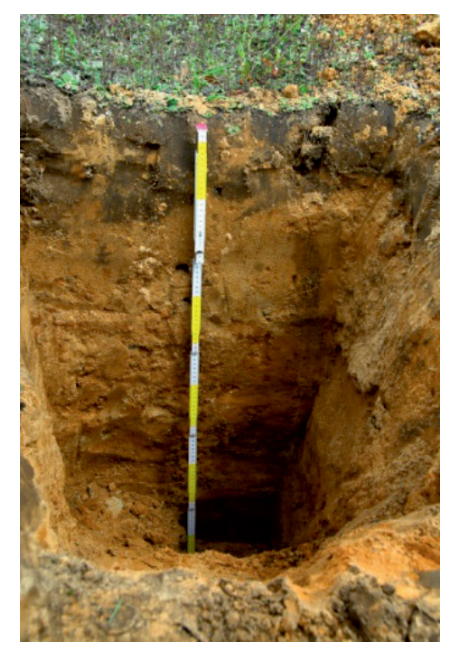

\author{
Depth Profile description \\ 1-0 cm - organic horizon \\ $0-16 \mathrm{~cm}$ - humus, medium sand, grey and dark brown \\ - rusty sand with single cobbles and stones of local and \\ $16-90 \mathrm{~cm}$ northern material, with dominating medium sand \\ $90-110 \mathrm{~cm}$ - loamy sand, light yellow with rusty (hardpan) stains
}

Fig. 5.44. Soil pit in study plot Raciszyn A (photo. E. Papińska, 2012) 
Table 5.25. Study plot Raciszyn A. Granulometric and chemical properties of soil

\begin{tabular}{|c|c|c|c|c|c|c|c|c|c|c|c|c|}
\hline \multirow[b]{2}{*}{ Soil pit } & \multirow{2}{*}{$\begin{array}{l}\text { Sampling } \\
\text { depth } \\
\mathrm{cm}\end{array}$} & \multicolumn{9}{|c|}{$\begin{array}{c}\text { Grain size distribution } \\
\%\end{array}$} & \multirow{2}{*}{$\begin{array}{l}\text { Gra- } \\
\text { nulo- } \\
\text { metric } \\
\text { sub- } \\
\text { group }\end{array}$} & \multirow{2}{*}{$\begin{array}{c}\text { Spe- } \\
\text { cific } \\
\text { surface } \\
\text { area } \\
\mathrm{m}^{2} \cdot \mathrm{g}-1\end{array}$} \\
\hline & & $\begin{array}{l}2.0- \\
1.0 \\
\mathrm{~mm} \\
\end{array}$ & $\begin{array}{l}1.0- \\
0.5 \\
\mathrm{~mm}\end{array}$ & $\begin{array}{l}0.5- \\
0.25 \\
\mathrm{~mm} \\
\end{array}$ & $\begin{array}{c}0.25- \\
0.1 \\
\mathrm{~mm}\end{array}$ & $\begin{array}{c}0.1- \\
0.05 \\
\mathrm{~mm} \\
\end{array}$ & $\begin{array}{l}0.05- \\
0.02 \\
\mathrm{~mm} \\
\end{array}$ & $\begin{array}{c}0.02- \\
0.005 \\
\mathrm{~mm}\end{array}$ & $\begin{array}{c}0.005- \\
0.002 \\
\mathrm{~mm} \\
\end{array}$ & $\begin{array}{c}<0.002 \\
\mathrm{~mm}\end{array}$ & & \\
\hline Raciszyn A & $0-20$ & 2.1 & 32.0 & 36.3 & 10.2 & 5.0 & 7.0 & 4.9 & 1.5 & 1.0 & ps & 0.150 \\
\hline Raciszyn A & $20-40$ & 0.7 & 28.3 & 38.4 & 12.6 & 5.1 & 7.0 & 4.8 & 1.7 & 1.4 & ps & 0.176 \\
\hline Raciszyn A & $90-110$ & 2.6 & 20.2 & 25.4 & 16.9 & 8.0 & 10.8 & 9.0 & 4.1 & 3.1 & pg & 0.363 \\
\hline
\end{tabular}

\begin{tabular}{|c|c|c|c|c|c|c|c|c|c|c|}
\hline \multirow{2}{*}{ Soil pit } & $\begin{array}{c}\text { Sampling } \\
\text { depth } \\
\end{array}$ & \multirow{2}{*}{$\begin{array}{c}\text { Humus } \\
\%\end{array}$} & \multirow{2}{*}{$\begin{array}{c}\mathrm{C} \text { total } \\
\%\end{array}$} & \multirow{2}{*}{$\begin{array}{c}\mathrm{N} \text { total } \\
\%\end{array}$} & \multirow{2}{*}{$\mathrm{C} / \mathrm{N}$} & \multicolumn{3}{|c|}{$\begin{array}{c}\mathrm{pH} \\
\text { in }\end{array}$} & \multicolumn{3}{|c|}{$\begin{array}{c}\text { Available nutrients } \\
\mathrm{mg} / 100 \mathrm{~g}\end{array}$} \\
\cline { 6 - 11 } & & & & $\mathrm{KCl}$ & $\mathrm{H}_{2} \mathrm{O}$ & $\mathrm{P}_{2} \mathrm{O}_{5}$ & $\mathrm{~K}_{2} \mathrm{O}$ & $\mathrm{Mg}$ \\
\hline Raciszyn A & $0-20$ & 0.94 & 0.545 & 0.05 & 10.905 & 4.2 & 4.8 & 3.0 & 1.9 & 0.3 \\
\hline Raciszyn A & $20-40$ & - & - & - & - & 4.3 & 5.2 & 0.5 & 2.2 & 0.4 \\
\hline Raciszyn A & $90-110$ & - & - & - & - & 4.1 & 5.3 & 1.0 & 8.0 & 3.2 \\
\hline
\end{tabular}

\begin{tabular}{|c|c|c|c|c|c|c|c|c|c|c|}
\hline \multirow{2}{*}{ Soil pit } & \multirow{2}{*}{$\begin{array}{l}\text { Sampling } \\
\text { depth } \\
\text { cm }\end{array}$} & \multirow{2}{*}{$\begin{array}{l}\text { Hydrolytic } \\
\text { acidity } \\
\text { mmol/100g }\end{array}$} & \multicolumn{4}{|c|}{$\begin{array}{l}\text { Exchangeable cations } \\
\text { me } / 100 \mathrm{~g}\end{array}$} & \multicolumn{4}{|c|}{$\begin{array}{l}\text { Sorption capacity } \\
\text { me } / 100 \mathrm{~g}\end{array}$} \\
\hline & & & $\mathrm{Ca}^{2+}$ & $\mathrm{Mg}^{2+}$ & $\mathrm{Na}^{+}$ & $\mathrm{K}^{+}$ & $S$ & $\mathrm{~T}$ & $(\mathrm{~T}-\mathrm{S})$ & $\mathrm{V}, \%$ \\
\hline Raciszyn A & $0-20$ & 3.21 & 0.15 & 0.023 & 0.017 & 0.062 & 0.252 & 3.462 & 3.21 & 7.279 \\
\hline Raciszyn A & $20-40$ & 1.70 & 0.20 & 0.032 & 0.017 & 0.067 & 0.316 & 2.016 & 1.70 & 15.675 \\
\hline Raciszyn A & $90-110$ & 2.12 & 2.05 & 0.270 & 0.061 & 0.213 & 2.594 & 4.714 & 2.12 & 55.028 \\
\hline
\end{tabular}

Source: own elaboration.

\section{Characteristics of the flora and fungi - Raciszyn A}

Approximately $100 \%$ of the study plot is covered with plants. Rich in species (31). Two species clearly dominate: Hieracium pilosella (about 50\%) and Agrostis capillaris (about 10\%). The share of other species ranges from $3 \%$ to $0.5 \%$ of area coverage. They include: Achillea millefolium, Arrthenatherum elatior, Artemisia campestris, Calamagrostis epigeios, Carex hirta, Carlina vulgaris, Cerastium arvense, Conyza canadensis, Fragaria viridis, Galium mollugo, Galium verum, Geum urbanum, Vicia angustifolia, Senecio jacobea (Photo 6.5). There are a small number of saplings of trees and shrubs:
Pinus sylvestris, Betula pendula and Pyrus communis (Tab. 6.1).

Fungi of the plot are abundant. They are represented by 19 species of macromycetes. The most frequently found species is Crinipellis scabella, which occurs on grasses, mainly on Agrostis capillaris. Among land fungi, the most abundant was Marasmius oreades. There were numerous sporocarps of mycorrhizal fungi in the plot: Scleroderma citrina, Amanita muscaria, Inocybe asterospora and Laccaria laccata (Photo 7.4) and others (Tab. 7.1). 
STUDY PLOT RACISZYN B (51 $05^{\prime} 26^{\prime \prime} \mathrm{N}, 18^{\circ} 51^{\prime} 11^{\prime \prime}$ E; elevation 214 m a.s.l. $)$
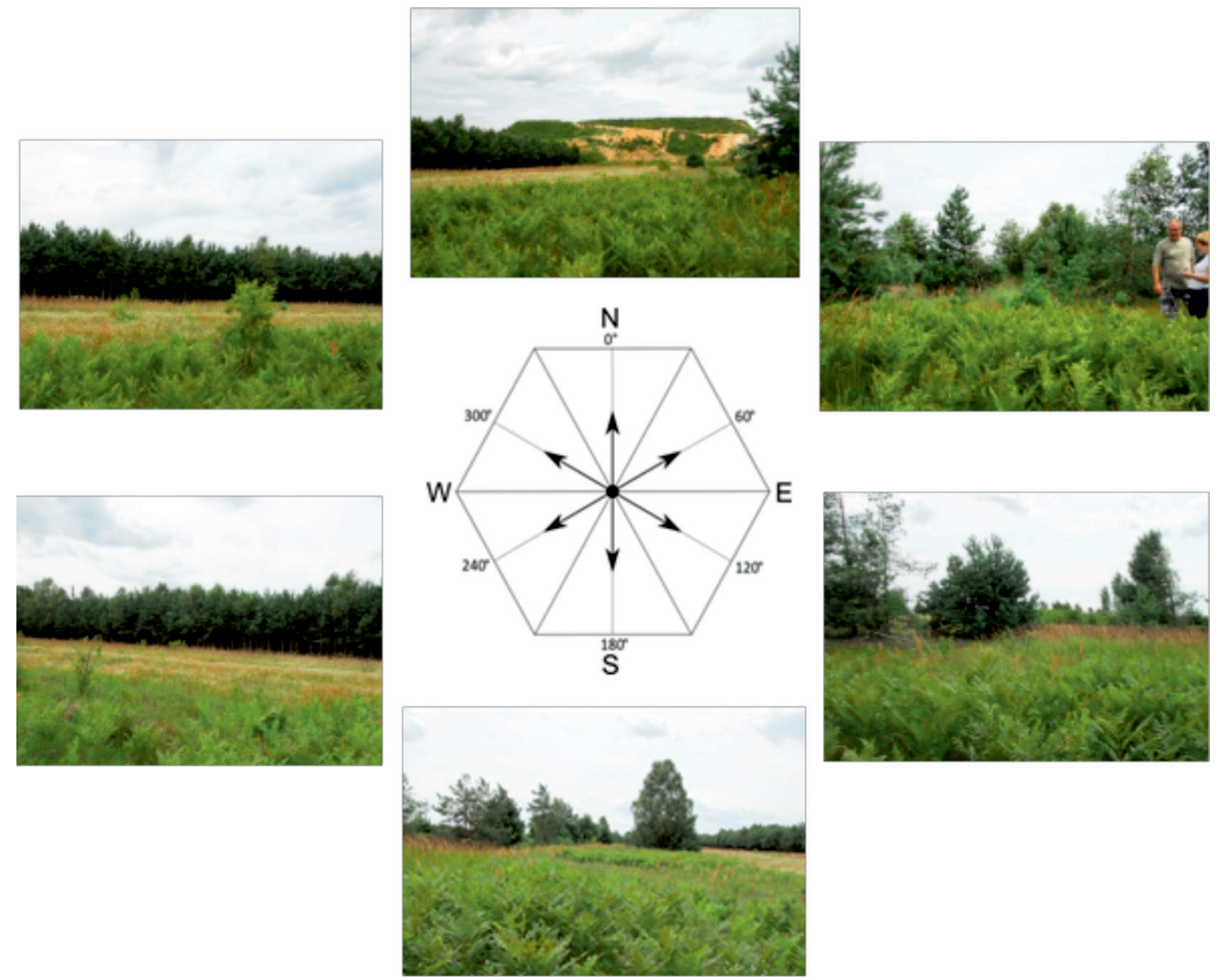

Fig. 5.45. Study plot Raciszyn B (photo E. Papińska, 2012)

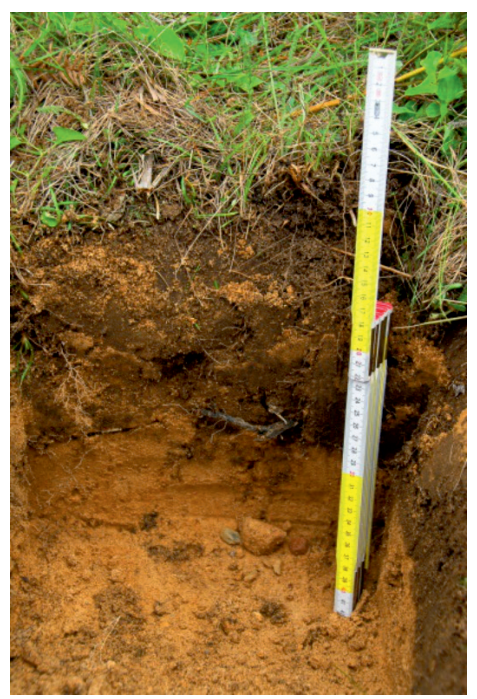

\footnotetext{
Depth Profile description

1-0 cm - organic horizon

0-20 cm - humus, medium sand, grey and dark brown

$20-90 \mathrm{~cm}$ - rusty sand with single cobbles and stones of local and northern material, with dominating medium sand

$90-110 \mathrm{~cm}$ - coarse sand, rusty
}

Fig. 5.46. Soil pit in study plot Raciszyn B (photo. E. Papińska, 2012) 
Table 5.26. Raciszyn B. Granulometric and chemical properties of soil

\begin{tabular}{|c|c|c|c|c|c|c|c|c|c|c|c|c|}
\hline \multirow[b]{2}{*}{ Soil pit } & \multirow{2}{*}{$\begin{array}{c}\text { Sampling } \\
\text { depth } \\
\text { cm }\end{array}$} & \multicolumn{9}{|c|}{$\begin{array}{c}\text { Grain size distribution } \\
\%\end{array}$} & \multirow{2}{*}{$\begin{array}{l}\text { Gra- } \\
\text { nulo- } \\
\text { metric } \\
\text { sub- } \\
\text { group }\end{array}$} & \multirow{2}{*}{$\begin{array}{c}\text { Spe- } \\
\text { cific } \\
\text { surface } \\
\text { area } \\
\mathrm{m}^{2} \cdot \mathrm{g}-\end{array}$} \\
\hline & & $\begin{array}{l}2.0- \\
1.0 \\
\mathrm{~mm}\end{array}$ & $\begin{array}{c}1.0- \\
0.5 \\
\mathrm{~mm}\end{array}$ & $\begin{array}{l}0.5- \\
0.25 \\
\mathrm{~mm}\end{array}$ & $\begin{array}{c}0.25- \\
0.1 \\
\mathrm{~mm}\end{array}$ & $\begin{array}{c}0.1- \\
0.05 \\
\mathrm{~mm}\end{array}$ & $\begin{array}{l}0.05- \\
0.02 \\
\mathrm{~mm}\end{array}$ & $\begin{array}{c}0.02- \\
0.005 \\
\mathrm{~mm}\end{array}$ & $\begin{array}{c}0.005- \\
0.002 \\
\mathrm{~mm}\end{array}$ & $\begin{array}{c}<0.002 \\
\mathrm{~mm}\end{array}$ & & \\
\hline Raciszyn B & $0-20$ & 2.3 & 33.1 & 37.5 & 9.6 & 4.9 & 6.1 & 4.4 & 1.2 & 0.8 & $\mathrm{pl}$ & 0.1230 \\
\hline Raciszyn B & $20-40$ & 3.5 & 37.4 & 40.3 & 6.7 & 1.4 & 2.8 & 4.3 & 2.1 & 1.4 & $\mathrm{pl}$ & 0.1680 \\
\hline Raciszyn B & $90-110$ & 2.4 & 31.7 & 45.4 & 14.4 & 0.4 & 1.3 & 2.4 & 1.4 & 0.6 & $\mathrm{pl}$ & 0.0887 \\
\hline
\end{tabular}

\begin{tabular}{|c|c|c|c|c|c|c|c|c|c|c|}
\hline \multirow[t]{2}{*}{ Soil pit } & \multirow{2}{*}{$\begin{array}{l}\text { Sampling } \\
\text { depth } \\
\text { cm }\end{array}$} & \multirow{2}{*}{$\begin{array}{c}\text { Humus } \\
\%\end{array}$} & \multirow{2}{*}{$\begin{array}{c}\text { C total } \\
\%\end{array}$} & \multirow{2}{*}{$\begin{array}{c}\mathrm{N} \text { total } \\
\%\end{array}$} & \multirow[t]{2}{*}{$\mathrm{C} / \mathrm{N}$} & \multicolumn{2}{|c|}{$\begin{array}{l}\mathrm{pH} \\
\text { in }\end{array}$} & \multicolumn{3}{|c|}{$\begin{array}{c}\text { Available nutrients } \\
\mathrm{mg} / 100 \mathrm{~g}\end{array}$} \\
\hline & & & & & & $\mathrm{KCl}$ & $\mathrm{H}_{2} \mathrm{O}$ & $\mathrm{P}_{2} \mathrm{O}_{5}$ & $\mathrm{~K}_{2} \mathrm{O}$ & $\mathrm{Mg}$ \\
\hline Raciszyn B & $0-20$ & 1.13 & 0.655 & 0.05 & 13.109 & 4.4 & 5.5 & 1.1 & 3.1 & 0.6 \\
\hline Raciszyn B & $20-40$ & - & - & - & - & 4.4 & 5.4 & 0.7 & 3.2 & 1.0 \\
\hline Raciszyn B & $90-110$ & - & - & - & - & 4.5 & 5.7 & 1.0 & 2.5 & 2.4 \\
\hline
\end{tabular}

\begin{tabular}{|c|c|c|c|c|c|c|c|c|c|c|}
\hline & \multirow{2}{*}{ Soil pit } & \multirow{2}{*}{$\begin{array}{c}\text { Sampling } \\
\text { depth } \\
\mathrm{cm}\end{array}$} & \multirow{2}{*}{$\begin{array}{c}\text { Hydrolytic } \\
\text { acidity } \\
\text { mmol/100g }\end{array}$} & \multicolumn{4}{|c|}{$\begin{array}{c}\text { Exchangeable cations } \\
\mathrm{me} / 100 \mathrm{~g}\end{array}$} & \multicolumn{4}{c|}{$\begin{array}{c}\text { Sorption capacity } \\
\text { me } / 100 \mathrm{~g}\end{array}$} \\
\cline { 5 - 13 } & & $\mathrm{Ca}^{2+}$ & $\mathrm{Mg}^{2+}$ & $\mathrm{Na}^{+}$ & $\mathrm{K}^{+}$ & $\mathrm{S}$ & $\mathrm{T}$ & $(\mathrm{T}-\mathrm{S})$ & $\mathrm{V}, \%$ \\
\hline Raciszyn B & $0-20$ & 2.51 & 0.8 & 0.048 & 0.035 & 0.102 & 0.985 & 3.495 & 2.51 & 28.183 \\
\hline Raciszyn B & $20-40$ & 1.27 & 1.2 & 0.078 & 0.043 & 0.097 & 1.418 & 2.688 & 1.27 & 52.753 \\
\hline Raciszyn B & $90-110$ & 0.73 & 1.1 & 0.190 & 0.043 & 0.067 & 1.400 & 2.130 & 0.73 & 65.728 \\
\hline
\end{tabular}

Source: own elaboration.

\section{Characteristics of the flora and fungi - Raciszyn B}

Approximately $100 \%$ of the study plot is covered with plants. Rich in species (31). Two species clearly dominate: Hieracium pilosella (about 50\%) and Agrostis capillaris (about 10\%). The share of other species ranges from $3 \%$ to $0.5 \%$ of area coverage. The other identified species of plants include: Achillea millefolium, Arrthenatherum elatius, Artemisia campestris, Calamagrostis epigeios, Carex hirta, Carlina vulgaris, Cerastium arvense, Conyza canadensis, Fragaria viridis, Galium mollugo, Galium verum. The plot also features a few sa- plings of trees and shrubs: Pinus sylvestris, Betula pendula and Pyrus communis (Tab. 6.1).

Fungi of the plot are abundant. They are represented by 14 species of macromycetes. Among land fungi, the most frequently found was $M a-$ rasmius oreades. Some sparse sporocarps of mycorrhizal fungi were also found in the area: Scleroderma citrina, Amanita muscaria, Inocybe asterospora, Xerocomus badius. On dead pine trees, sporocarps of Schizophyllum commune and Trichaptum abietinum were observed (Tab. 7.1). 
STUDY PLOT RACISZYN C ( $51^{\circ} 05^{\prime} 27^{\prime \prime} \mathrm{N}, 18^{\circ} 51^{\prime} 10^{\prime \prime}$; elevation $210 \mathrm{~m}$ a.s.l. $)$
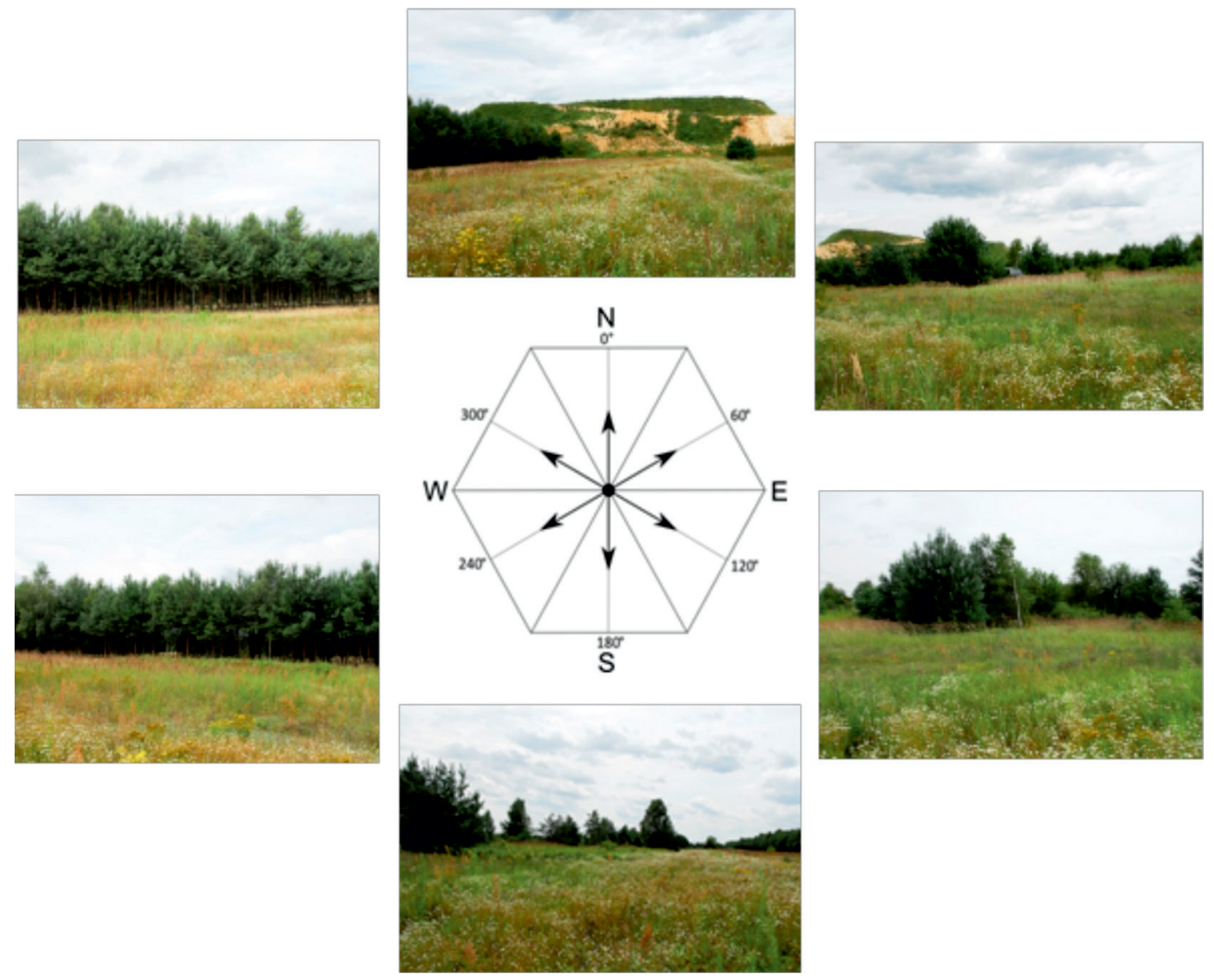

Fig. 5.47. Study plot Raciszyn C (photo E. Papińska, 2012)

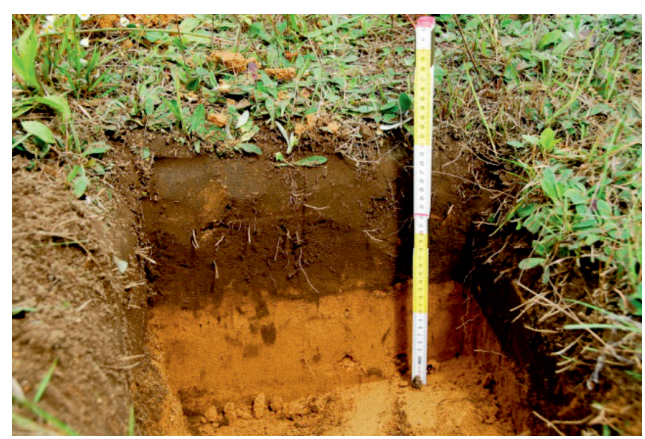

\author{
Depth Profile description \\ 1-0 cm - organic horizon \\ $0-20 \mathrm{~cm}$ - humus, medium sand, grey and dark brown \\ 20-100 cm - medium sand, rusty, moisture content increases \\ with depth
}

Fig. 5.48. Soil pit in study plot Raciszyn C (photo. E. Papińska, 2012) 
Table 5.27. Raciszyn C. Granulometric and chemical properties of soil

\begin{tabular}{|c|c|c|c|c|c|c|c|c|c|c|c|c|}
\hline \multirow[b]{2}{*}{ Soil pit } & \multirow{2}{*}{$\begin{array}{c}\text { Sampling } \\
\text { depth } \\
\mathrm{cm}\end{array}$} & \multicolumn{9}{|c|}{$\begin{array}{c}\text { Grain size distribution } \\
\%\end{array}$} & \multirow{2}{*}{$\begin{array}{l}\text { Gra- } \\
\text { nulo- } \\
\text { metric } \\
\text { sub- } \\
\text { group }\end{array}$} & \multirow{2}{*}{$\begin{array}{c}\text { Spe- } \\
\text { cific } \\
\text { surface } \\
\text { area } \\
\mathrm{m}^{2} \cdot \mathrm{g}^{-1}\end{array}$} \\
\hline & & $\begin{array}{l}2.0- \\
1.0 \\
\mathrm{~mm}\end{array}$ & $\begin{array}{c}1.0- \\
0.5 \\
\mathrm{~mm}\end{array}$ & $\begin{array}{l}0.5- \\
0.25 \\
\mathrm{~mm}\end{array}$ & $\begin{array}{c}0.25- \\
0.1 \\
\mathrm{~mm}\end{array}$ & $\begin{array}{l}0.1- \\
0.05 \\
\mathrm{~mm}\end{array}$ & $\begin{array}{l}0.05- \\
0.02 \\
\mathrm{~mm}\end{array}$ & $\begin{array}{c}0.02- \\
0.005 \\
\mathrm{~mm}\end{array}$ & $\begin{array}{c}0.005- \\
0.002 \\
\mathrm{~mm}\end{array}$ & $\begin{array}{c}<0.002 \\
\mathrm{~mm}\end{array}$ & & \\
\hline Raciszyn C & $0-20$ & 3.5 & 36.3 & 35.0 & 6.9 & 4.9 & 6.4 & 4.7 & 1.3 & 0.9 & ps & 0.131 \\
\hline Raciszyn C & $20-40$ & 2.6 & 43.4 & 43.4 & 6.1 & 1.4 & 1.0 & 1.4 & 0.6 & 0.1 & $\mathrm{pl}$ & 0.0401 \\
\hline Raciszyn C & $90-110$ & 1.7 & 37.1 & 41.8 & 8.5 & 3.2 & 3.3 & 2.5 & 1.1 & 0.7 & $\mathrm{pl}$ & 0.0952 \\
\hline
\end{tabular}

\begin{tabular}{|c|c|c|c|c|c|c|c|c|c|c|}
\hline \multirow[t]{2}{*}{ Soil pit } & \multirow{2}{*}{$\begin{array}{c}\text { Sampling } \\
\text { depth } \\
\mathrm{cm}\end{array}$} & \multirow{2}{*}{$\begin{array}{c}\text { Humus } \\
\%\end{array}$} & \multirow{2}{*}{$\begin{array}{c}\text { C total } \\
\%\end{array}$} & \multirow{2}{*}{$\begin{array}{l}\mathrm{N} \text { total } \\
\%\end{array}$} & \multirow[t]{2}{*}{$\mathrm{C} / \mathrm{N}$} & \multicolumn{2}{|c|}{$\begin{array}{l}\mathrm{pH} \\
\text { in }\end{array}$} & \multicolumn{3}{|c|}{$\begin{array}{c}\text { Available nutrients } \\
\mathrm{mg} / 100 \mathrm{~g}\end{array}$} \\
\hline & & & & & & $\mathrm{KCl}$ & $\mathrm{H}_{2} \mathrm{O}$ & $\mathrm{P}_{2} \mathrm{O}_{5}$ & $\mathrm{~K}_{2} \mathrm{O}$ & $\mathrm{Mg}$ \\
\hline Raciszyn C & $0-20$ & 1.73 & 1.003 & 0.078 & 12.865 & 4.2 & 4.9 & 2.5 & 1.7 & 0.3 \\
\hline Raciszyn C & $20-40$ & - & - & - & - & 4.6 & 5.4 & 1.6 & 0.2 & 0.4 \\
\hline Raciszyn C & $90-110$ & - & - & - & - & 4.8 & 5.9 & 1.2 & 1.9 & 0.6 \\
\hline
\end{tabular}

\begin{tabular}{|c|c|c|c|c|c|c|c|c|c|c|}
\hline & \multirow{2}{*}{ Soil pit } & \multirow{2}{*}{$\begin{array}{c}\text { Sampling } \\
\text { depth } \\
\text { cm }\end{array}$} & \multirow{2}{*}{$\begin{array}{c}\text { Hydrolytic } \\
\text { acidity } \\
\text { mmol/100g }\end{array}$} & \multicolumn{4}{|c|}{$\begin{array}{c}\text { Exchangeable cations } \\
\text { me/100g }\end{array}$} & \multicolumn{4}{c|}{$\begin{array}{c}\text { Sorption capacity } \\
\text { me/100g }\end{array}$} \\
\cline { 5 - 13 } & & $\mathrm{Ca}^{2+}$ & $\mathrm{Mg}^{2+}$ & $\mathrm{Na}^{+}$ & $\mathrm{K}^{+}$ & $\mathrm{S}$ & $\mathrm{T}$ & $(\mathrm{T}-\mathrm{S})$ & $\mathrm{V}, \%$ \\
\hline Raciszyn C & $0-20$ & 3.89 & 0.40 & 0.025 & 0.026 & 0.062 & 0.513 & 4.403 & 3.89 & 11.651 \\
\hline Raciszyn C & $20-40$ & 1.09 & 0.15 & 0.028 & 0.017 & 0.026 & 0.221 & 1.311 & 1.09 & 16.857 \\
\hline Raciszyn C & $90-110$ & 0.62 & 0.50 & 0.047 & 0.035 & 0.049 & 0.631 & 1.251 & 0.62 & 50.440 \\
\hline
\end{tabular}

Source: own elaboration.

\section{Characteristics of the flora and fungi - Raciszyn C}

Approximately $100 \%$ of the study plot is covered with plants. Rich in species (31). Two species dominate: Hieracium pilosella (about $40 \%$ ) and Pteridium aquilinum (30\%). About $5 \%$ of the area is covered by the following species: Achillea millefolium, Festuca rubra and Calamagrostis epigeios. The share of other species does not exceed $0.5 \%$ of area coverage (Photo 6.6). Other identified species of plants include: Agrostis capillaris, Artemisia vulgaris, Carlina vulgaris, Centaurea stoebe, C. scabiosa, Conyza canadensis, Convolvulus arven- sis, Coronilla varia, Dianthus carthusiana. There are also some trees and shrubs in the area: Pinus sylvestris and Rosa sp.

Fungi of the plot include 18 species of macromycetes (Photo 7.5). The most frequently found species is Marasmius oreades. In the lush undergrowth, the following species were also found: Clitopilus prunulus, Clitocybe vibecina, Conocybe tenera. Mycorrhizal fungi were represented by: Amanita muscaria and Boletus edulis. Hemimycena lactea was found fruiting on pine needles (Tab. 7.1).

\section{Analysis of granulometric composition and chemical properties of soils in the Raciszyn study plot group}

The analysis results presented in tables 5.25-5.27 indicate that grain size distribution at individual plots of the study plot group is diverse. At plot Raciszyn A, the granulometric composition of all samples is dominated by the fraction of sands, whose share decreases with depth, from $85.6 \%$ in the sample from $0-20 \mathrm{~cm}$ to $73.1 \%$ at the depth of $90-110 \mathrm{~cm}$. On the other hand, there is an increasing share of silts (from $13.4 \%$ to $23.9 \%$ ) and clays (from $1 \%$ to $3.1 \%$ ). Content analysis of the 
fractions $<0.02 \mathrm{~mm}$, which is the basis for assigning the agronomic category of soils, ranges from $7.4 \%$ in the sample from $0-20 \mathrm{~cm}$ to $16.2 \%$ at the depth of $90-110 \mathrm{~cm}$. The soil was classified as category I - very light soils.

The high content of the finest fractions (silts and clays) in all samples, influences another parameter of the analysed soil - its specific surface area. The value of this parameter increases with depth, from $0.15 \mathrm{~m}^{2} \cdot \mathrm{g}^{-1}$ (sample from the depth of $0-20 \mathrm{~cm}$ ) to $0.363 \mathrm{~m}^{2} \cdot \mathrm{g}^{-1}$ (sample from the depth of $90-110 \mathrm{~cm}$ ).

At plot Raciszyn B, the granulometric composition in all horizons is similar - the granulometric subgroup of loose sand (pl). In this case, the share of the sandy fraction increases with depth from $87.4(0-20 \mathrm{~cm})$ to $94.3(90-100 \mathrm{~cm}$ of depth). The share of silts is the highest in the uppermost sample and reaches nearly $12 \%$, to decrease to the value of $5.1 \%$ in the deepest sample. The share of the clayey fraction at this site is the highest at the depth of $20-40 \mathrm{~cm}: 1.4 \%$. Dominance of the sandy fraction in the grain size distribution resulted in classifying them as agronomic category I - very light soils. The specific surface area is the lowest at the depth of 90-100 cm with the value of $0.0887 \mathrm{~m}^{2} \cdot \mathrm{g}^{-1}$, and the highest at $0.176 \mathrm{~m}^{2} \cdot \mathrm{g}^{-1}$ in the sample from the depth of $20-40 \mathrm{~cm}$, where the share of the finest fraction (clays) is the highest of the entire profile.

The grain size distribution results for plot Raciszyn $\mathrm{C}$ are different. The occurrence of slightly loamy sand (ps) was identified the closest to the surface, and in other horizons - loose sands (pl). The content of sands is the lowest in the sample from the depth of $20-40 \mathrm{~cm}$ is nearly $97 \%$, which leaves very little room for other granulometric groups $-3 \%$ of silts and $0.1 \%$ of clays. Specific surface reached the lowest value in this horizon: $0.0401 \mathrm{~m}^{2} \cdot \mathrm{g}^{-1}$. More favourable parameters of grain size distribution, and thus also of specific surface, were found in the sample from the 0-20 cm horizon. The share of the sandy fraction of $87.4 \%$ is the lowest of the entire profile, whereas it is the highest for silts (12.4\%) and clays $(0.9 \%)$. In this horizon, the value of specific surface was estimated at $0.131 \mathrm{~m}^{2} \cdot \mathrm{g}^{-1}$.

The $\mathrm{pH}$ reaction of the soil in $\mathrm{KCl}$ in all horizons of plots Raciszyn A and B is very acidic and changes with depth within a very narrow range. It is similar in the $0-20 \mathrm{~cm}$ horizon at Raciszyn C, but it increases slightly to acidic in the subsequent two horizons. The same parameter analysed in $\mathrm{H}_{2} \mathrm{O}$ indicates a slight increase into the depth of the profile, e.g.: from pH 4.8 to 5.3 at site $\mathrm{A}$, or from $\mathrm{pH} 4.9$ to 5.9 at site $\mathrm{C}$. The degree of saturation with alkaline cations (V) also corresponds to this tendency, increasing in samples from Raciszyn A from $7.279 \%$ in the upper horizon to $55.028 \%$ in the deepest horizon. This is due to the content of calcium cations $\left(\mathrm{Ca}^{2+}\right)$, which increases with depth. However, the highest saturation with alkaline cations occurs in samples from Raciszyn B. This parameter changes with depth from $28.183 \%(0-20 \mathrm{~cm})$ to $65.728 \%(90-100 \mathrm{~cm})$.

The content of nutrients available to plants is not sufficient. For phosphorus $\left(\mathrm{P}_{2} \mathrm{O}_{5}\right)$, the value is low in only one sample (the first sample from Raciszyn A), and very low in all the remaining samples. Potassium $\left(\mathrm{K}_{2} \mathrm{O}\right)$ also shows low or very low content. The values were a bit better for magnesium (Mg), from very low to medium availability (samples from the depth of $90-110 \mathrm{~cm}$ at sites Raciszyn A and B).

The humus content in the uppermost horizon ranges from $0.94 \%$ (at Raciszyn A) to $1.73 \%$ (at Raciszyn C). It is the highest content of all the analysed study plots. The content of organic carbon ranges from $0.545 \%$ (at Raciszyn A) to $1.003 \%$. 


\title{
5.3. Abandoned land study plot groups in the buffer zones around the Bolimów Landscape Park and the Łódź Hills Landscape Park
}

\author{
Anna Majchrowska, Jolanta Adamczyk, Jarosław Sieradzki
}

\section{POLESIE STUDY PLOT GROUP}

The Polesie study plot group lies about $5 \mathrm{~km}$ to the west of the Bolimów Landscape Park, in Łyszkowice Commune, Łowicz District. According to the physicogeographical regionalisation of Poland (Kondracki 2002), it is located in the central part of the mesoregion of the Łowicz-Błonie Plain, in the macroregion of the Central Mazovian Lowland. The Polesie group lies in one of the southern branches of the North-Central Ecological Corridor (Jędrzejewski et al. 2011).

The area has the structure of a mosaic of abandoned lands at various ages and planted forests, mostly pine ones. In the west, it borders a vast forest complex, whereas on the other sides, it is surrounded with areas of alternating farmlands, abandoned lands and forests, which are mostly artificially planted. The share of farmlands clearly increases towards the north-east and east.

The geological substratum of the study plot group was presented at the junction of two sheets of the Detailed Geological Map of Poland (Brzeziński 1990, 1995), as fluvioglacial and fluvial sands and gravels of the North-Mazovian stage of the Central Polish Glaciation, and upper fluvioglacial sands and gravels of the Warta Glaciation, respectively. The grade of soils was described in soil and agricultural maps (http://geoportal.lodzkie. $\mathrm{pl} / \mathrm{imap} /$ ) as loose sands, of which leached brown soils originated. The soils were classified as very poor rye complex (7) of agricultural suitability and class VI of soil valuation (http://geoportal. lodzkie.pl/imap/).

All plots of the described group: Polesie A, $B$ and $C$ are found in a type 6 geocomplex - glacial and fluvioglacial boulders, cobbles, gravels, sands and muds of plateaus, alluvial fans and erosional-accumulational terraces. It is included in the group of lithogenic geocomplexes, which normally do not undergo excessive moisturising, associated with permeable Quaternary formations.

The physiognomy of the study plot group and soil profiles is illustrated with figures 5.49-5.54. The results of laboratory analyses of soils were presented in tables 5.28-5.30.

STUDY PLOT POLESIE A (5000'799N, 195 59'941E, elevation 112 m a.s.l.)
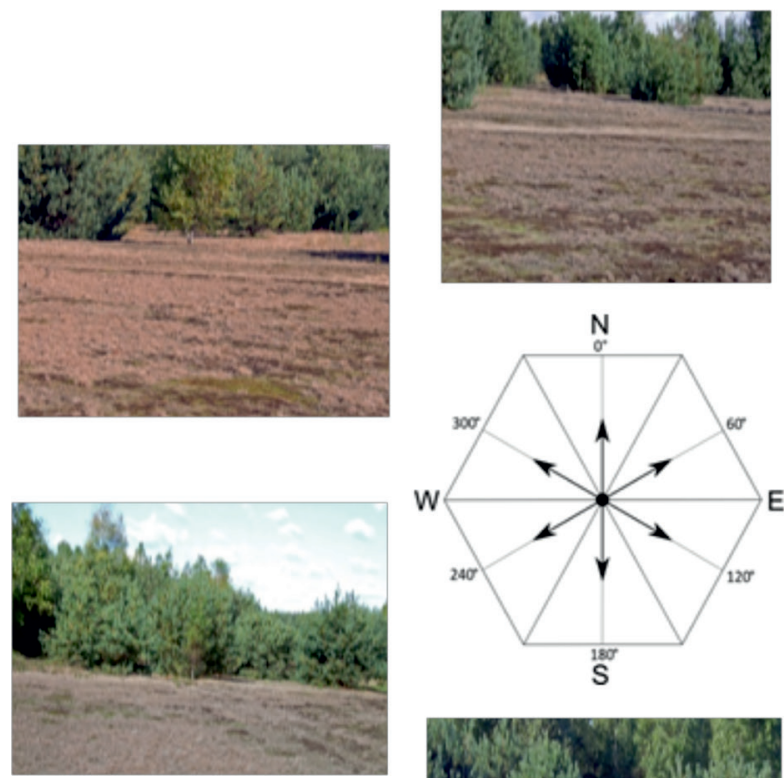
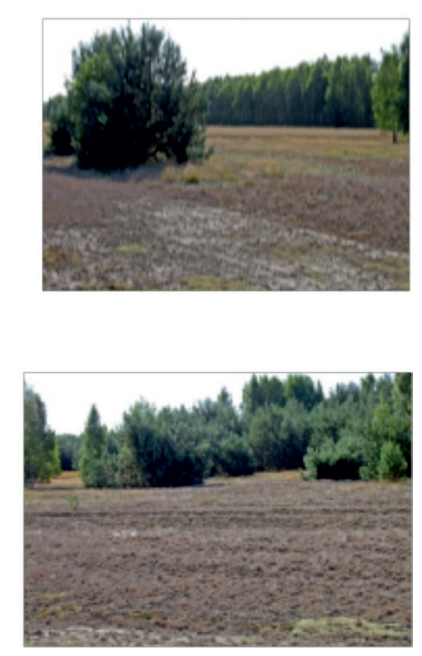

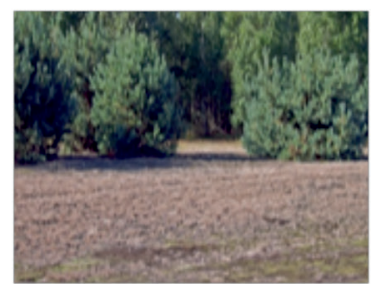

Fig. 5.49. Study plot Polesie A (photo A. Majchrowska, 2013) 


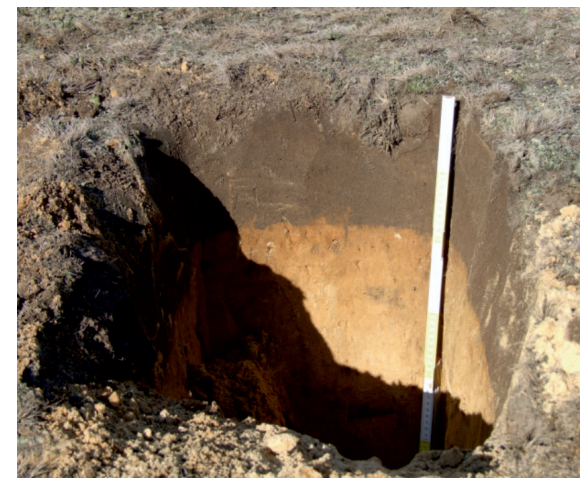

Depth Profile description

0-20 cm - humus, fine silty sand, dark brown

0-100 cm - fine and medium sand, yellow

$100-140 \mathrm{~cm}$ - fine and medium sand, light yellow and grey

$140-150 \mathrm{~cm}$ - medium and fine sand, light yellow and grey, with some single cobbles of $1-1.5 \mathrm{~cm}$ in diameter

Fig. 5.50. Soil pit in study plot Polesie A (photo A. Majchrowska, 2013)

Table 5.28. Study plot Polesie A. Granulometric and chemical properties of the soil

\begin{tabular}{|c|c|c|c|c|c|c|c|c|c|c|c|c|}
\hline \multirow[b]{2}{*}{ Soil pit } & \multirow{2}{*}{$\begin{array}{c}\text { Sampling } \\
\text { depth } \\
\text { cm }\end{array}$} & \multicolumn{9}{|c|}{$\begin{array}{c}\text { Grain size distribution } \\
\%\end{array}$} & \multirow{2}{*}{$\begin{array}{c}\text { Granu- } \\
\text { lome- } \\
\text { tric } \\
\text { sub- } \\
\text { group }\end{array}$} & \multirow{2}{*}{$\begin{array}{c}\text { Spe- } \\
\text { cific } \\
\text { surface } \\
\text { area } \\
\mathrm{m}^{2} \cdot \mathrm{g}^{-1}\end{array}$} \\
\hline & & $\begin{array}{l}2.0- \\
1.0 \\
\mathrm{~mm}\end{array}$ & $\begin{array}{c}1.0- \\
0.5 \\
\mathrm{~mm}\end{array}$ & $\begin{array}{l}0.5- \\
0.25 \\
\mathrm{~mm}\end{array}$ & $\begin{array}{c}0.25- \\
0.1 \\
\mathrm{~mm}\end{array}$ & $\begin{array}{l}0.1- \\
0.05 \\
\mathrm{~mm}\end{array}$ & $\begin{array}{l}0.05- \\
0.02 \\
\mathrm{~mm}\end{array}$ & $\begin{array}{c}0.02- \\
0.005 \\
\mathrm{~mm}\end{array}$ & $\begin{array}{c}0.005- \\
0.002 \\
\mathrm{~mm}\end{array}$ & $\begin{array}{c}<0.002 \\
\mathrm{~mm}\end{array}$ & & \\
\hline Polesie A & $0-20$ & 1.3 & 20.7 & 30.4 & 15.5 & 2.9 & 5.2 & 11.7 & 7.5 & 4.8 & gp & 0.532 \\
\hline Polesie A & $20-40$ & 1.7 & 24.2 & 41.6 & 27.2 & 3.2 & 1.1 & 0.7 & 0.2 & 0.0 & $\mathrm{p}$ & 0.0313 \\
\hline Polesie A & $90-110$ & 1.0 & 21.7 & 42.4 & 30.8 & 3.0 & 1.1 & 0.0 & 0.0 & 0.0 & $\mathrm{p}$ & 0.0244 \\
\hline
\end{tabular}

\begin{tabular}{|c|c|c|c|c|c|c|c|c|c|c|}
\hline \multirow[t]{2}{*}{ Soil pit } & \multirow{2}{*}{$\begin{array}{c}\text { Sampling } \\
\text { depth } \\
\text { cm }\end{array}$} & \multirow{2}{*}{$\begin{array}{c}\text { Humus } \\
\%\end{array}$} & \multirow{2}{*}{$\begin{array}{l}\text { C total } \\
\%\end{array}$} & \multirow{2}{*}{$\begin{array}{l}\mathrm{N} \text { total } \\
\%\end{array}$} & \multirow[t]{2}{*}{$\mathrm{C} / \mathrm{N}$} & \multicolumn{2}{|c|}{$\begin{array}{l}\mathrm{pH} \\
\text { in }\end{array}$} & \multicolumn{3}{|c|}{$\begin{array}{c}\text { Available nutrients } \\
\mathrm{mg} / 100 \mathrm{~g}\end{array}$} \\
\hline & & & & & & $\mathrm{KCl}$ & $\mathrm{H}_{2} \mathrm{O}$ & $\mathrm{P}_{2} \mathrm{O}_{5}$ & $\mathrm{~K}_{2} \mathrm{O}$ & $\mathrm{Mg}$ \\
\hline Polesie A & $0-20$ & 1.42 & 0.824 & 0.053 & 15.54 & 4.0 & 4.4 & 9.8 & 0.4 & 0.3 \\
\hline Polesie A & $20-40$ & - & - & - & - & 4.5 & 4.7 & 2.5 & 0.3 & 0.2 \\
\hline Polesie A & $90-110$ & - & - & - & - & 4.6 & 4.9 & 1.4 & 0.3 & 0.3 \\
\hline
\end{tabular}

\begin{tabular}{|c|c|c|c|c|c|c|c|c|c|c|}
\hline \multirow[t]{2}{*}{ Soil pit } & \multirow{2}{*}{$\begin{array}{c}\text { Sampling } \\
\text { depth } \\
\mathrm{cm}\end{array}$} & \multirow{2}{*}{$\begin{array}{l}\text { Hydrolytic } \\
\text { acidity } \\
\text { mmol/100g }\end{array}$} & \multicolumn{4}{|c|}{$\begin{array}{c}\text { Exchangeable cations } \\
\text { me } / 100 \mathrm{~g}\end{array}$} & \multicolumn{4}{|c|}{$\begin{array}{c}\text { Sorption capacity } \\
\mathrm{me} / 100 \mathrm{~g}\end{array}$} \\
\hline & & & $\mathrm{Ca}^{2+}$ & $\mathrm{Mg}^{2+}$ & $\mathrm{Na}^{+}$ & $\mathrm{K}^{+}$ & S & $\mathrm{T}$ & $(\mathrm{T}-\mathrm{S})$ & $\mathrm{V}, \%$ \\
\hline Polesie A & $0-20$ & 3.62 & 0.15 & 0.017 & 0.026 & 0.026 & 0.219 & 3.839 & 3.62 & 5.7 \\
\hline Polesie A & $20-40$ & 1.39 & 0.15 & 0.013 & 0.017 & 0.018 & 0.198 & 1.588 & 1.39 & 12.5 \\
\hline Polesie A & $90-110$ & 1.00 & 0.10 & 0.013 & 0.017 & 0.018 & 0.148 & 1.148 & 1.00 & 12.9 \\
\hline
\end{tabular}

Source: own elaboration.

\section{Characteristics of the flora and fungi - Polesie A}

The study plot is floristically poor, only about $15 \%$ is covered with plants. Only three species of vascular plants were found: Corynephorus canescens, which covered about $10 \%$ of the area, Rumex acetosella and Pinus sylvestris in small amounts. The layer of lichens (about 3\%) and mosses (about $1 \%$ ) was not very abundant (Photo 7.6). The following species of lichens were found: Cladonia arbuscula, Cladonia coccifera, Cladonia uncialis.
Among the lichens there was one species of moss - Polytrichum piliferum (Tab. 6.1).

Fungi of the plot are sparse. They are represented by only 4 species of macromycetes. The most frequently occurring was Marasmius oreades. Several sporocarps of mycorrhizal fungi were also observed: Scleroderma citrina, Amanita muscaria, Inocybe asterospora (Tab. 7.1). 
STUDY PLOT POLESIE B (5200'857N, 195 59'921E, elevation 103 m a.s.l.)
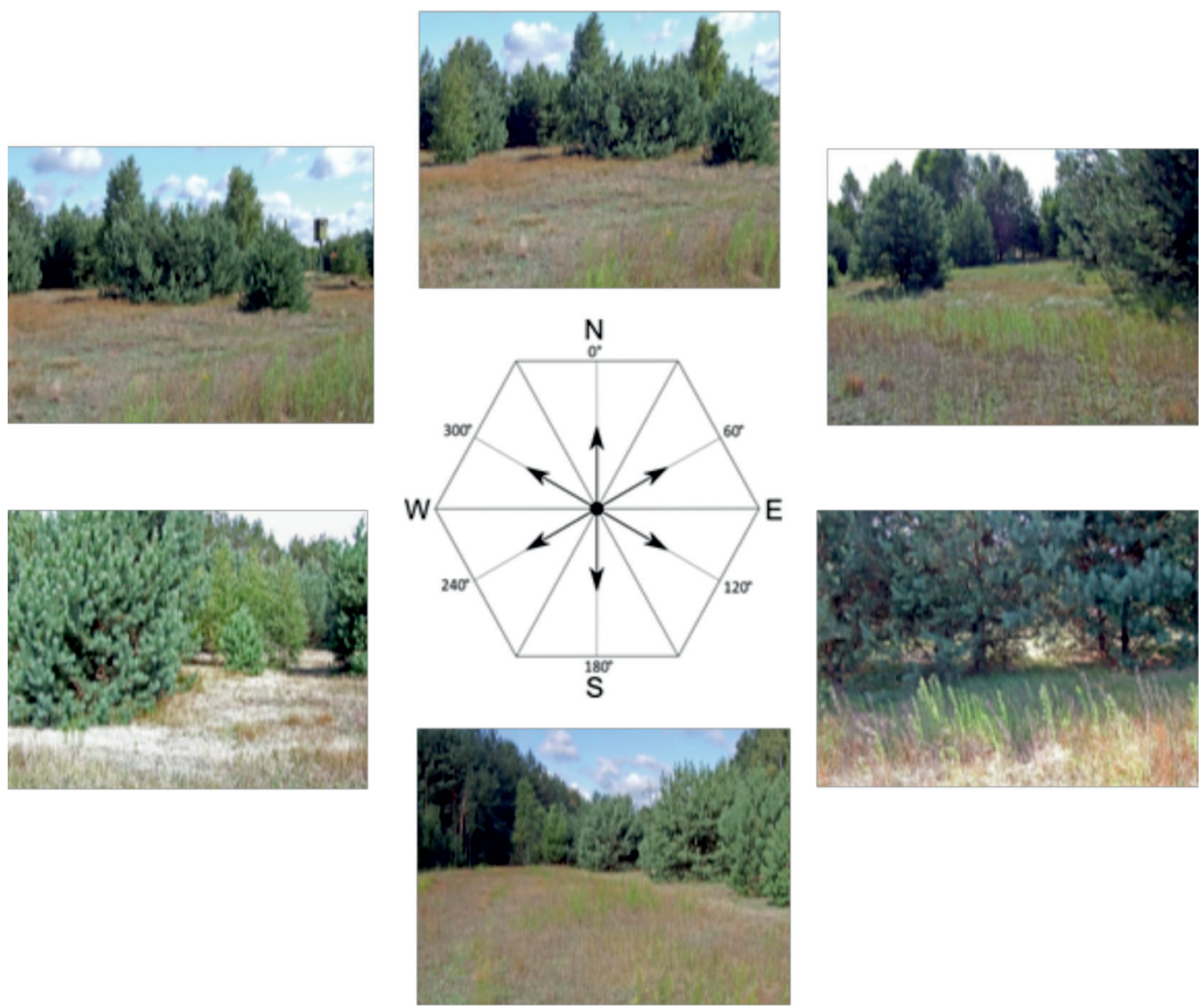

Fig. 5.51. Study plot Polesie B (photo A. Majchrowska, 2013)

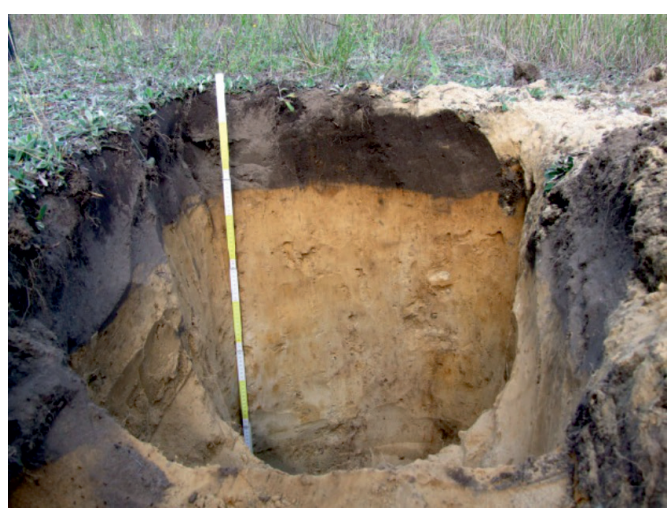

Depth Profile description

$0-25 \mathrm{~cm}$ - humus, medium and fine sand, dark grey 25-50 cm - medium and fine sand, golden, with gravel 50-100 cm - medium sand, light yellow, with more gravel $100-140 \mathrm{~cm}$ - medium sand, light beige, with gravel

Fig. 5.52. Soil pit in study plot Polesie B (photo A. Majchrowska, 2013) 
Table 5.29. Study plot Polesie B. Granulometric and chemical properties of soil

\begin{tabular}{|c|c|c|c|c|c|c|c|c|c|c|c|c|}
\hline \multirow[b]{2}{*}{ Soil pit } & \multirow{2}{*}{$\begin{array}{c}\text { Sampling } \\
\text { depth } \\
\mathrm{cm}\end{array}$} & \multicolumn{9}{|c|}{$\begin{array}{c}\text { Grain size distribution } \\
\%\end{array}$} & \multirow{2}{*}{$\begin{array}{l}\text { Gra- } \\
\text { nulo- } \\
\text { metric } \\
\text { sub- } \\
\text { group }\end{array}$} & \multirow{2}{*}{$\begin{array}{c}\text { Spe- } \\
\text { cific } \\
\text { surface } \\
\text { area } \\
\mathrm{m}^{2} \cdot \mathrm{g}^{-1}\end{array}$} \\
\hline & & $\begin{array}{l}2.0- \\
1.0 \\
\mathrm{~mm}\end{array}$ & $\begin{array}{c}1.0- \\
0.5 \\
\mathrm{~mm}\end{array}$ & $\begin{array}{l}0.5- \\
0.25 \\
\mathrm{~mm}\end{array}$ & $\begin{array}{c}0.25- \\
0.1 \\
\mathrm{~mm}\end{array}$ & $\begin{array}{l}0.1- \\
0.05 \\
\mathrm{~mm}\end{array}$ & $\begin{array}{l}0.05- \\
0.02 \\
\mathrm{~mm}\end{array}$ & $\begin{array}{c}0.02- \\
0.005 \\
\mathrm{~mm}\end{array}$ & $\begin{array}{c}0.005- \\
0.002 \\
\mathrm{~mm}\end{array}$ & $\begin{array}{c}<0.002 \\
\mathrm{~mm}\end{array}$ & & \\
\hline Polesie B & $0-20$ & 0.6 & 21.5 & 39.6 & 25 & 4.9 & 4.7 & 2.8 & 0.8 & 0.1 & $\mathrm{p}$ & 0.0645 \\
\hline Polesie B & $20-40$ & 0.5 & 20.6 & 40.9 & 28.8 & 3.4 & 2.7 & 2.0 & 0.8 & 0.3 & $\mathrm{p}$ & 0.0697 \\
\hline Polesie B & $90-110$ & 2.1 & 30.7 & 42.8 & 20.3 & 1.0 & 0.8 & 1.3 & 0.8 & 0.2 & $\mathrm{p}$ & 0.0515 \\
\hline
\end{tabular}

\begin{tabular}{|c|c|c|c|c|c|c|c|c|c|c|}
\hline \multirow{2}{*}{ Soil pit } & \multirow{2}{*}{$\begin{array}{c}\text { Sampling } \\
\text { depth } \\
\text { cm }\end{array}$} & \multirow{2}{*}{$\begin{array}{c}\text { Humus } \\
\%\end{array}$} & \multirow{2}{*}{$\begin{array}{c}\text { C total } \\
\%\end{array}$} & \multirow{2}{*}{$\begin{array}{l}\mathrm{N} \text { total } \\
\%\end{array}$} & \multirow[t]{2}{*}{$\mathrm{C} / \mathrm{N}$} & \multicolumn{2}{|c|}{$\begin{array}{l}\mathrm{pH} \\
\text { in }\end{array}$} & \multicolumn{3}{|c|}{$\begin{array}{c}\text { Available nutrients } \\
\mathrm{mg} / 100 \mathrm{~g}\end{array}$} \\
\hline & & & & & & $\mathrm{KCl}$ & $\mathrm{H}_{2} \mathrm{O}$ & $\mathrm{P}_{2} \mathrm{O}_{5}$ & $\mathrm{~K}_{2} \mathrm{O}$ & $\mathrm{Mg}$ \\
\hline Polesie B & $0-20$ & 2.24 & 1.3 & 0.092 & 14.12 & 4.0 & 4.6 & 10.3 & 1.1 & 0.6 \\
\hline Polesie B & $20-40$ & - & - & - & - & 4.5 & 5.0 & 1.7 & 0.8 & 0.4 \\
\hline Polesie B & $90-110$ & - & - & - & - & 4.6 & 4.9 & 1.5 & 1.0 & 0.4 \\
\hline
\end{tabular}

\begin{tabular}{|c|c|c|c|c|c|c|c|c|c|c|}
\hline \multirow[t]{2}{*}{ Soil pit } & \multirow{2}{*}{$\begin{array}{c}\text { Sampling } \\
\text { depth } \\
\text { cm }\end{array}$} & \multirow{2}{*}{$\begin{array}{l}\text { Hydrolytic } \\
\text { acidity } \\
\text { mmol/100g }\end{array}$} & \multicolumn{4}{|c|}{$\begin{array}{c}\text { Exchangeable cations } \\
\text { me } / 100 \mathrm{~g}\end{array}$} & \multicolumn{4}{|c|}{$\begin{array}{c}\text { Sorption capacity } \\
\text { me/100g }\end{array}$} \\
\hline & & & $\mathrm{Ca}^{2+}$ & $\mathrm{Mg}^{2+}$ & $\mathrm{Na}^{+}$ & $\mathrm{K}^{+}$ & S & $\mathrm{T}$ & $(\mathrm{T}-\mathrm{S})$ & $\mathrm{V}, \%$ \\
\hline Polesie B & $0-20$ & 4.90 & 0.35 & 0.045 & 0.026 & 0.044 & 0.465 & 5.365 & 4.90 & 11.5 \\
\hline Polesie B & $20-40$ & 2.00 & 0.15 & 0.030 & 0.017 & 0.038 & 0.235 & 2.235 & 2.00 & 9.5 \\
\hline Polesie B & $90-110$ & 1.27 & 0.15 & 0.018 & 0.017 & 0.044 & 0.229 & 1.499 & 1.27 & 15.3 \\
\hline
\end{tabular}

Source: own elaboration.

\section{Characteristics of the flora and fungi - Polesie B}

Approximately $90 \%$ of the study plot is covered with plants. Three species dominate among plants: Agrostis capillaris (about 10\%), Hieracium pilosella (about 60\%) and Festuca rubra (about $10 \%)$. The share of other species does not exceed $3 \%$ of area coverage. Other identified species include: Apera spica-venti, Jasione montana, Centaurea cyanus, Conyza canadensis, Elymus repens, Galeopsis ladanum (Tab. 6.1).
Fungi of the plot are sparse. They are represented by only 9 species of macromycetes. The most frequently found species is Crinipellis scabella, which occurs on grasses. Among land fungi, the most abundant species were Marasmius oreades and Bovista plumbea (Tab. 7.1). 
STUDY PLOT POLESIE C (5200'893N, 1959'895E, elevation 106 m a.s.l. $)$
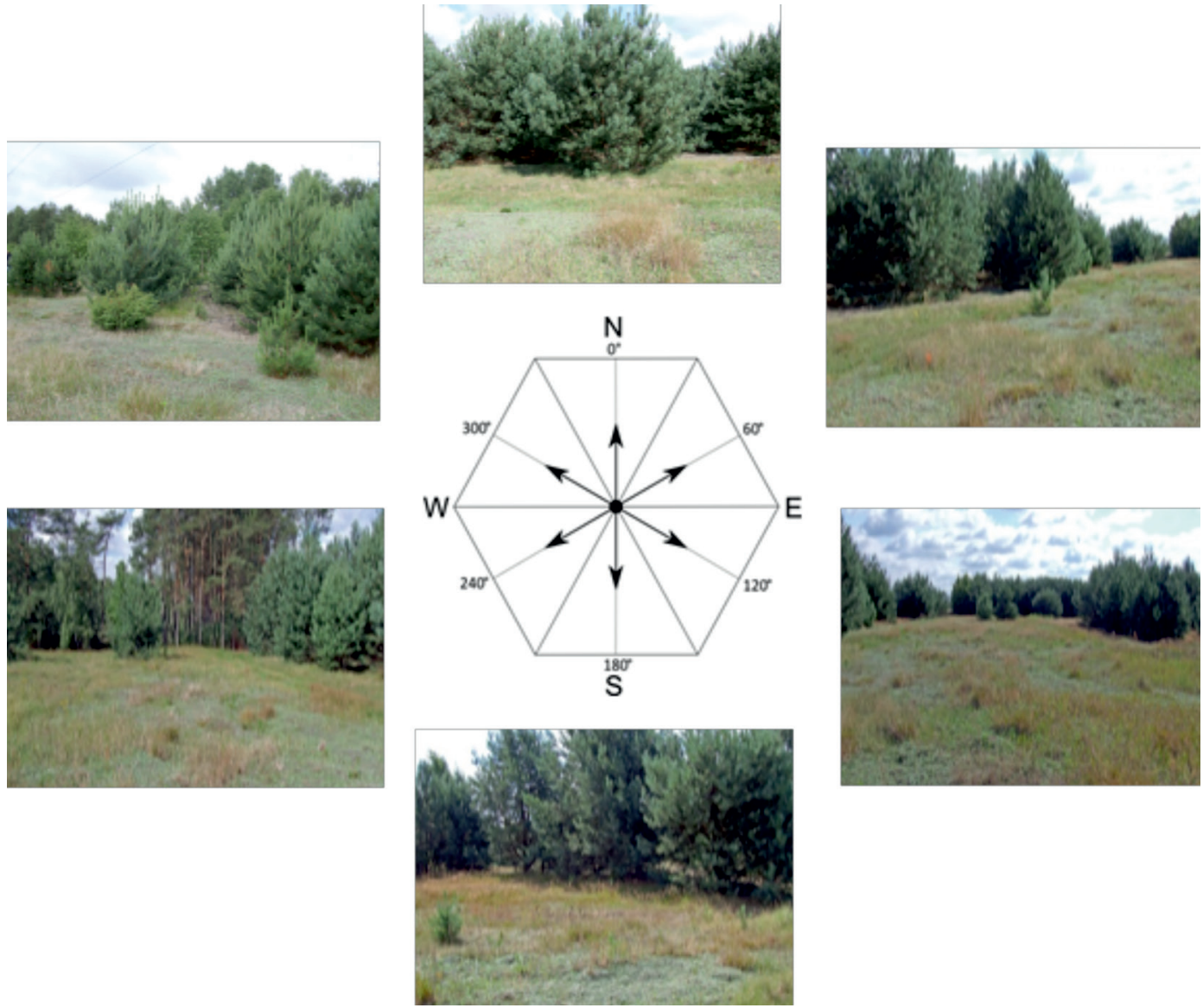

Fig. 5.53. Study plot Polesie C (photo A. Majchrowska, 2013)

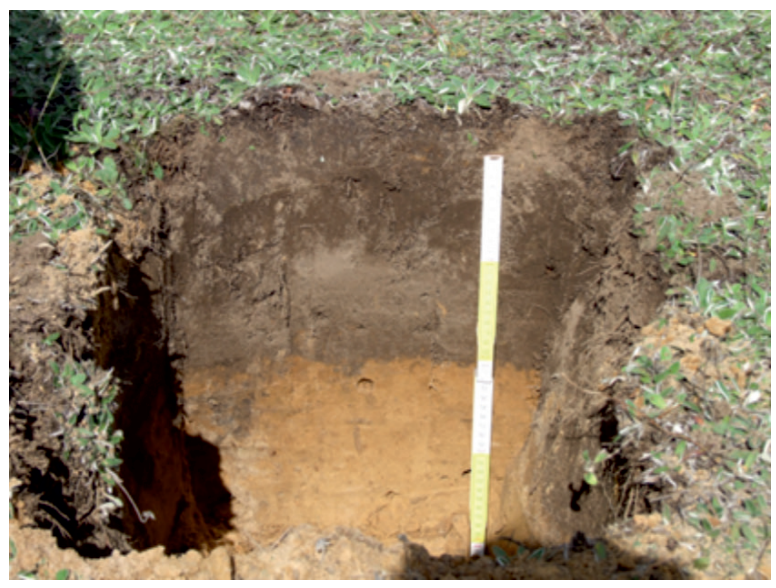

Depth Profile description

0-26 cm - humus, medium and fine sand, dark brown

26-40 cm - fine sand, yellow and golden, with a single cobble of $8 \mathrm{~cm}$ in diameter

40-90 cm - fine and medium sand, yellow and golden

90-140 cm - medium and coarse sand, light yellow, with gravel

Fig. 5.54. Soil pit in study plot Polesie C (photo A. Majchrowska, 2013) 
Table 5.30. Study plot Polesie C. Granulometric and chemical properties of soil

\begin{tabular}{|c|c|c|c|c|c|c|c|c|c|c|c|c|}
\hline \multirow[b]{2}{*}{ Soil pit } & \multirow{2}{*}{$\begin{array}{c}\text { Sampling } \\
\text { depth } \\
\text { cm }\end{array}$} & \multicolumn{9}{|c|}{$\begin{array}{c}\text { Grain size distribution } \\
\%\end{array}$} & \multirow{2}{*}{$\begin{array}{l}\text { Gra- } \\
\text { nulo- } \\
\text { metric } \\
\text { sub- } \\
\text { group }\end{array}$} & \multirow{2}{*}{$\begin{array}{c}\text { Spe- } \\
\text { cific } \\
\text { surface } \\
\text { area } \\
\mathrm{m}^{2} \cdot \mathrm{g}^{-1}\end{array}$} \\
\hline & & $\begin{array}{l}2.0- \\
1.0 \\
\mathrm{~mm}\end{array}$ & $\begin{array}{c}1.0- \\
0.5 \\
\mathrm{~mm}\end{array}$ & $\begin{array}{l}0.5- \\
0.25 \\
\mathrm{~mm}\end{array}$ & $\begin{array}{c}0.25- \\
0.1 \\
\mathrm{~mm}\end{array}$ & $\begin{array}{l}0.1- \\
0.05 \\
\mathrm{~mm}\end{array}$ & $\begin{array}{l}0.05- \\
0.02 \\
\mathrm{~mm}\end{array}$ & $\begin{array}{c}0.02- \\
0.005 \\
\mathrm{~mm}\end{array}$ & $\begin{array}{c}0.005- \\
0.002 \\
\mathrm{~mm}\end{array}$ & $\begin{array}{c}<0.002 \\
\mathrm{~mm}\end{array}$ & & \\
\hline Polesie C & $0-20$ & 1.5 & 23.9 & 37.3 & 20.7 & 5.6 & 5.9 & 3.7 & 1.0 & 0.5 & $\mathrm{p}$ & 0.0954 \\
\hline Polesie C & $20-40$ & 0.6 & 25.1 & 43.2 & 19.9 & 2.6 & 3.3 & 3.0 & 1.4 & 0.9 & $\mathrm{p}$ & 0.1190 \\
\hline Polesie C & $90-110$ & 11.0 & 51.1 & 34.6 & 3.3 & 0.0 & 0.0 & 0.0 & 0.0 & 0.0 & $\mathrm{p}$ & 0.0116 \\
\hline
\end{tabular}

\begin{tabular}{|c|c|c|c|c|c|c|c|c|c|c|}
\hline \multirow[t]{2}{*}{ Soil pit } & \multirow{2}{*}{$\begin{array}{c}\text { Sampling } \\
\text { depth } \\
\text { cm }\end{array}$} & \multirow{2}{*}{$\begin{array}{c}\text { Humus } \\
\%\end{array}$} & \multirow{2}{*}{$\begin{array}{c}\mathrm{C} \text { total } \\
\%\end{array}$} & \multirow{2}{*}{$\begin{array}{c}\mathrm{N} \text { total } \\
\%\end{array}$} & \multirow{2}{*}{$\mathrm{C} / \mathrm{N}$} & \multicolumn{2}{|c|}{$\begin{array}{l}\mathrm{pH} \\
\text { in }\end{array}$} & \multicolumn{3}{|c|}{$\begin{array}{c}\text { Available nutrients } \\
\mathrm{mg} / 100 \mathrm{~g}\end{array}$} \\
\hline & & & & & & $\mathrm{KCl}$ & $\mathrm{H}_{2} \mathrm{O}$ & $\mathrm{P}_{2} \mathrm{O}_{5}$ & $\mathrm{~K}_{2} \mathrm{O}$ & $\mathrm{Mg}$ \\
\hline Polesie C & $0-20$ & 2.23 & 1.29 & 0.093 & 13.91 & 4.6 & 5.5 & 5.2 & 1.1 & 0.8 \\
\hline Polesie C & $20-40$ & - & - & - & - & 4.7 & 5.7 & 1.6 & 0.8 & 0.4 \\
\hline Polesie C & $90-110$ & - & - & - & - & 4.7 & 5.6 & 2.0 & 0.6 & 0.2 \\
\hline
\end{tabular}

\begin{tabular}{|c|c|c|c|c|c|c|c|c|c|c|}
\hline & \multirow{2}{*}{ Soil pit } & \multirow{2}{*}{$\begin{array}{c}\text { Sampling } \\
\text { depth } \\
\mathrm{cm}\end{array}$} & \multirow{2}{*}{$\begin{array}{c}\text { Hydrolytic } \\
\text { acidity } \\
\text { mmol/100g }\end{array}$} & \multicolumn{4}{|c|}{$\begin{array}{c}\text { Exchangeable cations } \\
\mathrm{me} / 100 \mathrm{~g}\end{array}$} & \multicolumn{4}{c|}{$\begin{array}{c}\text { Sorption capacity } \\
\text { me/100g }\end{array}$} \\
\cline { 6 - 14 } & & $\mathrm{Ca}^{2+}$ & $\mathrm{Mg}^{2+}$ & $\mathrm{Na}^{+}$ & $\mathrm{K}^{+}$ & $\mathrm{S}$ & $\mathrm{T}$ & $(\mathrm{T}-\mathrm{S})$ & $\mathrm{V}, \%$ \\
\hline Polesie C & $0-20$ & 3.49 & 1.80 & 0.060 & 0.043 & 0.044 & 1.947 & 5.437 & 3.49 & 35.80 \\
\hline Polesie C & $20-40$ & 1.90 & 0.50 & 0.028 & 0.026 & 0.044 & 0.598 & 2.498 & 1.90 & 23.94 \\
\hline Polesie C & $90-110$ & 0.90 & 0.10 & 0.015 & 0.035 & 0.026 & 0.176 & 1.076 & 0.90 & 16.36 \\
\hline
\end{tabular}

Source: own elaboration.

\section{Characteristics of the flora and fungi - Polesie C}

Approximately $90 \%$ of the study plot is covered with plants. Three species dominate among plants: Hieracium pilosella (about 50\%), Agrostis capillaris (about 10\%) and Corynephorus canescens (about 10\%). The share of other species does not exceed $2 \%$ of area coverage. Other identified species include: Helichrysum arenaria, Conyza canadensis, Elymus repens, Festuca rubra, Holcus lanatus, Viola arvensis. Species found in the young tree layer include: Pinus sylvestris and Sorbus aucuparia (Tab. 6.1).
Fungi of the area are poor. They are represented by only 6 species of macromycetes. The most frequently occurring species is Crinipellis scabella, which occurs on grasses, mainly on Agrostis capillaris and Festuca rubra. Among terrestrial fungi, the most abundant species was Marasmius oreades. Fairly numerous sporocarps of mycorrhizal fungi were found in the area: Amanita muscaria, Xerocomus badius (Tab. 7.1).

\section{Analysis of granulometric composition and chemical properties of soils in the Polesie study plot group}

On the basis of the results of grain size analyses (Tab. 5.28-5.30), soils of the abandoned lands in the Polesie study plot group were classified as the granulometric group of sand (p) (Polskie Towarzystwo Gleboznawcze 2009). The surface hori- zon $(0-20 \mathrm{~cm})$ of soil at plot Polesie A was an exception, with an increased content of the silty and clayey fractions (up to 29.2\%), which was classified as sandy loam (gp). At all plots, the content of this fraction decreased with depth. The grain size 
distribution of soil at plot Polesie A allowed for classifying it as the medium category of agrotechnical heaviness, and the soils at the other plots were classified as very light soils.

The value of specific surface area shows a relation with the granulometric composition and was at its highest where the content of fine fractions was high, in the surface horizon of plot Polesie A.

The content of humus, ranging from 1.42 to $2.24 \%$ in the analysed soils, was defined as medium and high. The poorest in humus was the soil at plot Polesie $\mathrm{A}$. The $\mathrm{C} / \mathrm{N}$ ratio oscillated around 15:1-13:1. The $\mathrm{pH}$ reaction of the analysed soils at plots Polesie A and B was very acidic, and at site Polesie C - acidic.

The surface horizon of soils in the Polesie study plot group was characterised by low and medium content of easily available phosphorus, whereas in the lower horizons, the content of phosphorus decreased to very low. The content of available potassium and magnesium was very low for all the analysed soil horizons.

The soils revealed varied hydrolytic acidity, with values ranging from $0.9-4.9 \mathrm{mmol} / 100 \mathrm{~g}$. The total capacity for cation exchange was very low: the maximum value was $5.437 \mathrm{mmol} / 100 \mathrm{~g}$. Saturation of the sorptive complex with alkaline ions was very low at plots Polesie A and B $(5.7 \%-15.3 \%)$. The degree of saturation with alkalis at plot Polesie $C$ in the $0-20 \mathrm{~cm}$ horizon was the highest, though it was low in general, as it was $35.8 \%$ and decreased into the depth of the soil profile to $16 \%$. The highest values of acidity, total alkaline cations and the values of total absorbing capacity were found in humus horizons.

\section{WOLA MAKOWSKA STUDY PLOT GROUP}

The Wola Makowska study plot group is located about $7 \mathrm{~km}$ west of the Bolimów Landscape Park, in Maków Commune, Skierniewice District. According to the physicogeographical regionalisation (Kondracki 2002), it is located in the southern part of the mesoregion of the Łowicz-Błonie Plain, in the macroregion of the Central Mazovian Lowland. Elevation of the analysed area is about 110-120 $\mathrm{m}$ a.s.l.

In the west, the study plot group borders on an extensive forest complex. In the north and east, it borders on belts of farmlands and meadows, dissected by a stream called Ruczaj. In the south, the area neighbours arable lands with a small share of abandoned fields, and with several strips of planted woodland.

According to the Detailed Geological Map of Poland, the substratum of the analysed area is formed by Quaternary sands of alluvial fans and Holocene aeolian sands (Balińska-Wuttke 1958). On the basis of a soil and agricultural map (http:// geoportal.lodzkie.pl/imap/), the soils were qualified as leeched brown soils, formed upon loose sand and were classified as very poor rye complex
(7) of agricultural suitability. The plot Makowska Wola $C$ lies in direct neighbourhood of the border between leeched brown soils and muck soils, classified as very poor grassland (3z). Soils of the plots Wola Makowska A and B were qualified as valuation class VIRz, and soils of the plot Wola Makowska C - as class VI of arable lands (http:// geoportal.lodzkie.pl/imap/).

Plot Wola Makowska A is located within a type 15 geocomplex - aeolian sands of dunes and shields, plot B - on the border between geocomplexes 15 and 6 - geocomplex of glacial and fluvioglacial boulders, cobbles, gravels, sands and muds of plateaus, alluvial fans and erosional-accumulational terraces, and plot Wola Makowska C - in a type 6 geocomplex. Type 6 and 15 geocomplexes are included in the group of lithogenic units, which normally do not undergo excessive moisturising, associated with permeable Quaternary deposits.

Photographical documentation of the Wola Makowska study plot group and soil profiles consists of a set of figures 5.55-5.60. The results of detailed laboratory analyses are presented in tables 5.31-5.33. 


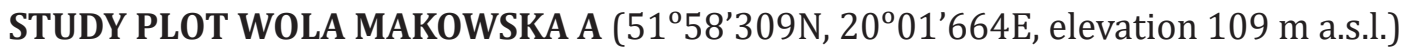
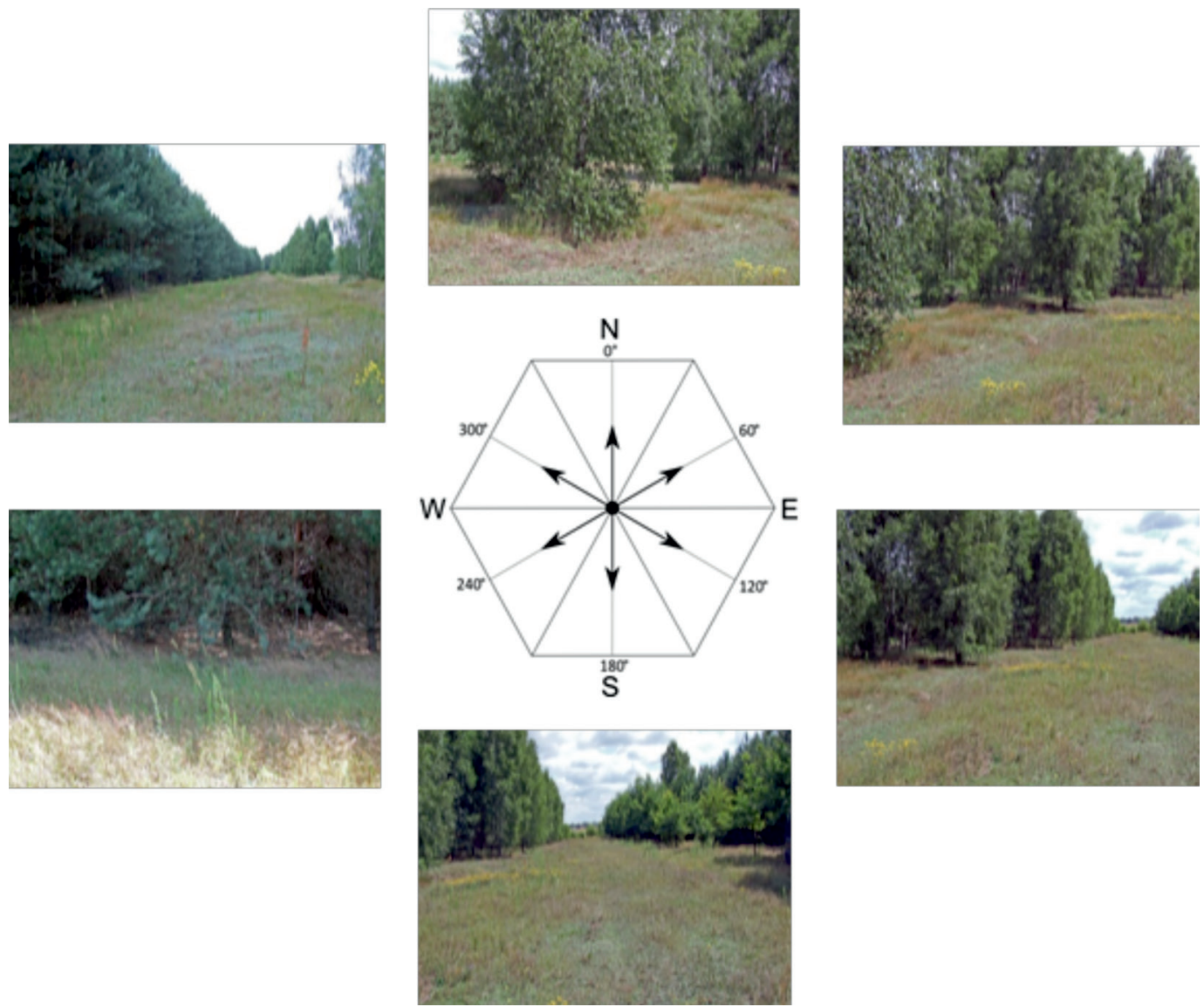

Fig. 5.55. Study plot Wola Makowska A (photo A. Majchrowska, 2013)

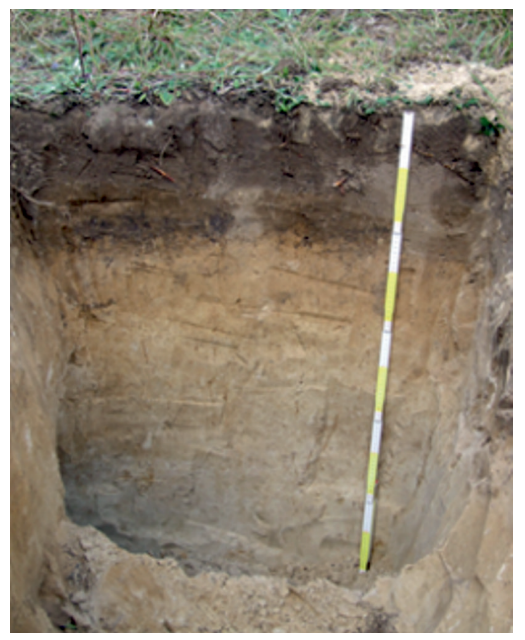

Depth Profile description

0-20 cm - humus; unsorted sand, silty, light brown 20-60 cm - fine and silty sand, yellow and beige $60-110 \mathrm{~cm}$ - silty sand and fine sand, light beige $110-150 \mathrm{~cm}$ - silty sand, light grey and white

Fig. 5.56. Soil pit in study plot Wola Makowska A (photo A. Majchrowska, 2013) 
Table 5.31. Study plot Wola Makowska A. Granulometric and chemical properties of soil

\begin{tabular}{|c|c|c|c|c|c|c|c|c|c|c|c|c|}
\hline \multirow[b]{2}{*}{ Soil pit } & \multirow{2}{*}{$\begin{array}{c}\text { Sampling } \\
\text { depth } \\
\mathrm{cm}\end{array}$} & \multicolumn{9}{|c|}{$\begin{array}{c}\text { Grain size distribution } \\
\%\end{array}$} & \multirow{2}{*}{$\begin{array}{l}\text { Gra- } \\
\text { nulo- } \\
\text { metric } \\
\text { sub- } \\
\text { group }\end{array}$} & \multirow{2}{*}{$\begin{array}{l}\text { Spe- } \\
\text { cific } \\
\text { surface } \\
\text { area } \\
\mathrm{m}^{2} \cdot \mathrm{g}^{-1}\end{array}$} \\
\hline & & $\begin{array}{l}2.0- \\
1.0 \\
\mathrm{~mm}\end{array}$ & $\begin{array}{c}1.0- \\
0.5 \\
\mathrm{~mm}\end{array}$ & $\begin{array}{l}0.5- \\
0.25 \\
\mathrm{~mm}\end{array}$ & $\begin{array}{c}0.25- \\
0.1 \\
\mathrm{~mm}\end{array}$ & $\begin{array}{c}0.1- \\
0.05 \\
\mathrm{~mm}\end{array}$ & $\begin{array}{l}0.05- \\
0.02 \\
\mathrm{~mm}\end{array}$ & $\begin{array}{c}0.02- \\
0.005 \\
\mathrm{~mm}\end{array}$ & $\begin{array}{c}0.005- \\
0.002 \\
\mathrm{~mm}\end{array}$ & $\begin{array}{c}<0.002 \\
\mathrm{~mm}\end{array}$ & & \\
\hline $\begin{array}{l}\text { Wola Ma- } \\
\text { kowska A }\end{array}$ & $0-20$ & 0.1 & 14.7 & 41.6 & 32.6 & 4.9 & 2.8 & 2.0 & 0.9 & 0.4 & $\mathrm{p}$ & 0.0794 \\
\hline $\begin{array}{l}\text { Wola Ma- } \\
\text { kowska A }\end{array}$ & $20-40$ & 0.0 & 12.4 & 39.4 & 34.4 & 7.1 & 3.5 & 2.0 & 0.8 & 0.5 & $\mathrm{p}$ & 0.0826 \\
\hline $\begin{array}{l}\text { Wola Ma- } \\
\text { kowska A }\end{array}$ & $90-110$ & 0.0 & 10.0 & 37.7 & 38.3 & 8.7 & 3.2 & 1.4 & 0.6 & 0.1 & $\mathrm{p}$ & 0.0562 \\
\hline
\end{tabular}

\begin{tabular}{|c|c|c|c|c|c|c|c|c|c|c|}
\hline Soil pit & $\begin{array}{c}\text { Sampling } \\
\text { depth } \\
\mathrm{cm}\end{array}$ & $\begin{array}{c}\text { Humus } \\
\%\end{array}$ & $\begin{array}{c}\mathrm{C} \text { total } \\
\%\end{array}$ & $\begin{array}{c}\mathrm{N} \text { total } \\
\%\end{array}$ & $\mathrm{C} / \mathrm{N}$ & \multicolumn{3}{|c|}{$\begin{array}{c}\mathrm{pH} \\
\mathrm{in}\end{array}$} & \multicolumn{3}{|c|}{$\begin{array}{c}\text { Available nutrients } \\
\mathrm{mg} / 100 \mathrm{~g}\end{array}$} \\
\cline { 6 - 11 } \\
$\begin{array}{c}\text { Wola Ma- } \\
\text { kowska A }\end{array}$ & $0-20$ & 0.63 & 0.365 & 0.033 & 11.07 & 4.3 & 5.3 & 10.1 & 2.0 & 0.4 \\
\hline $\begin{array}{c}\text { Wola Ma- } \\
\text { kowska A }\end{array}$ & $20-40$ & - & - & - & - & 4.5 & 5.1 & 17.2 & 0.7 & 0.3 \\
\hline $\begin{array}{c}\text { Wola Ma- } \\
\text { kowska A }\end{array}$ & $90-110$ & - & - & - & - & 4.5 & 5.6 & 6.6 & 1.1 & 0.7 \\
\hline
\end{tabular}

\begin{tabular}{|c|c|c|c|c|c|c|c|c|c|c|}
\hline \multirow[t]{2}{*}{ Soil pit } & \multirow{2}{*}{$\begin{array}{l}\text { Sampling } \\
\text { depth } \\
\mathrm{cm}\end{array}$} & \multirow{2}{*}{$\begin{array}{l}\text { Hydrolytic } \\
\text { acidity } \\
\text { mmol } / 100 \mathrm{~g}\end{array}$} & \multicolumn{4}{|c|}{$\begin{array}{c}\text { Exchangeable cations } \\
\text { me } / 100 \mathrm{~g}\end{array}$} & \multicolumn{4}{|c|}{$\begin{array}{l}\text { Sorption capacity } \\
\text { me } / 100 \mathrm{~g}\end{array}$} \\
\hline & & & $\mathrm{Ca}^{2+}$ & $\mathrm{Mg}^{2+}$ & $\mathrm{Na}^{+}$ & $\mathrm{K}^{+}$ & S & $\mathrm{T}$ & $(\mathrm{T}-\mathrm{S})$ & $\mathrm{V}, \%$ \\
\hline $\begin{array}{l}\text { Wola Ma- } \\
\text { kowska A }\end{array}$ & $0-20$ & 2.20 & 0.50 & 0.025 & 0.026 & 0.074 & 0.625 & 2.825 & 2.20 & 22.12 \\
\hline $\begin{array}{l}\text { Wola Ma- } \\
\text { kowska A }\end{array}$ & $20-40$ & 2.00 & 0.20 & 0.015 & 0.026 & 0.031 & 0.272 & 2.272 & 2.00 & 11.97 \\
\hline $\begin{array}{l}\text { Wola Ma- } \\
\text { kowska A }\end{array}$ & $90-110$ & 1.00 & 0.30 & 0.048 & 0.009 & 0.038 & 0.395 & 1.395 & 1.00 & 28.32 \\
\hline
\end{tabular}

Source: own elaboration.

\section{Characteristics of the flora and fungi - Wola Makowska A}

Approximately $90 \%$ of the study plot is covered with plants. Poor in species (11). Four species dominate: Hieracium pilosella (40\%), Rumex acetosa $(10 \%)$, Corynephorus canescens $(10 \%)$ and Chelichrysum arenaria (10\%). The share of other species ranges between $5 \%$ and $0.5 \%$ of area covera- ge. They include: Achillea millefolium, Convolvulus arvensis, Conyza canadensis (Tab. 6.1).

Fungi of the plot are sparse. They are represented by only 3 species of macromycetes. They are: Marasmius oreades, Conocybe rickeaniana and Panaeolus foenisecii (Tab. 7.1). 


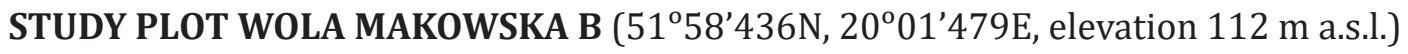
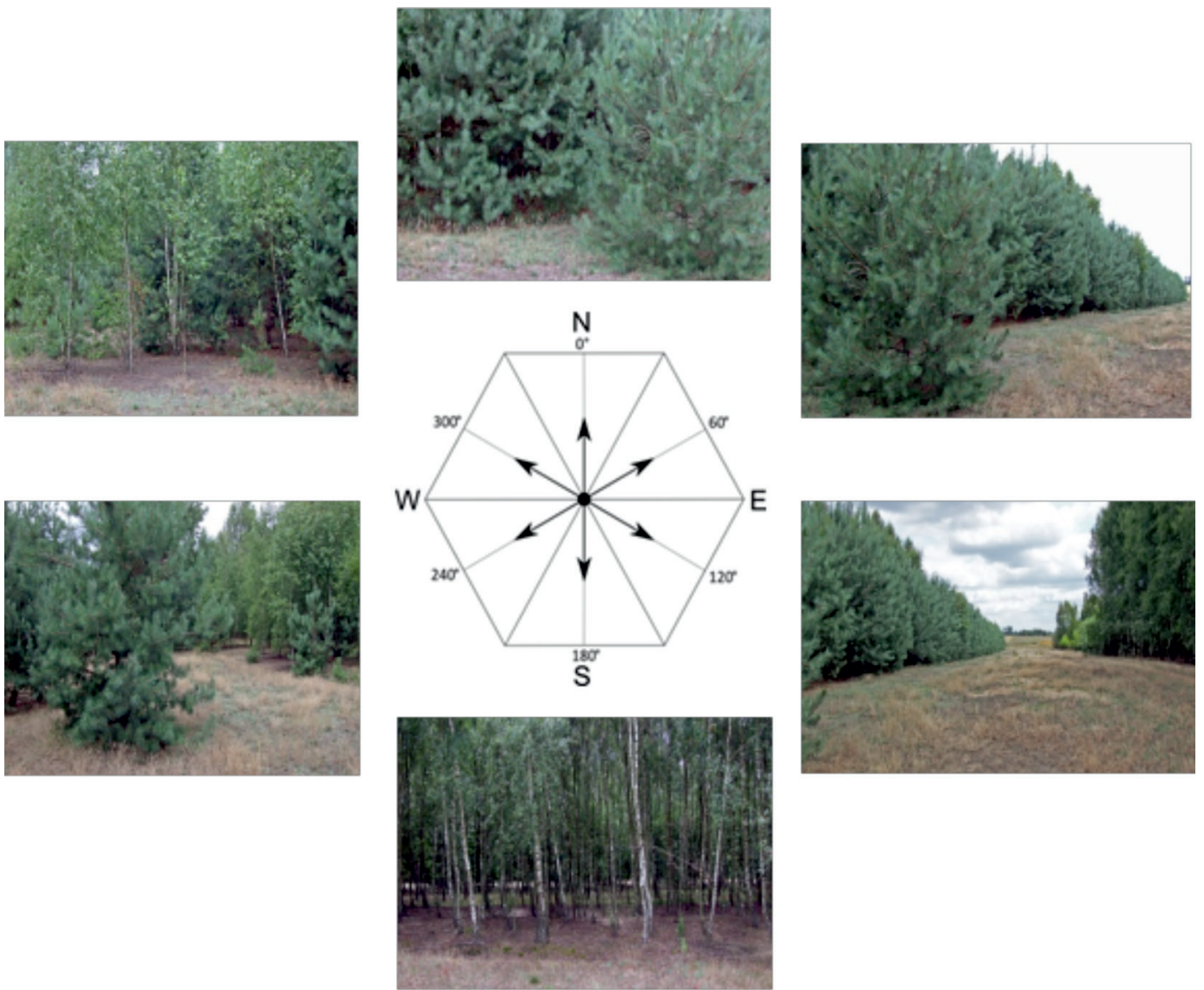

Fig. 5.57. Study plot Wola Makowska B (photo A. Majchrowska, 2013)

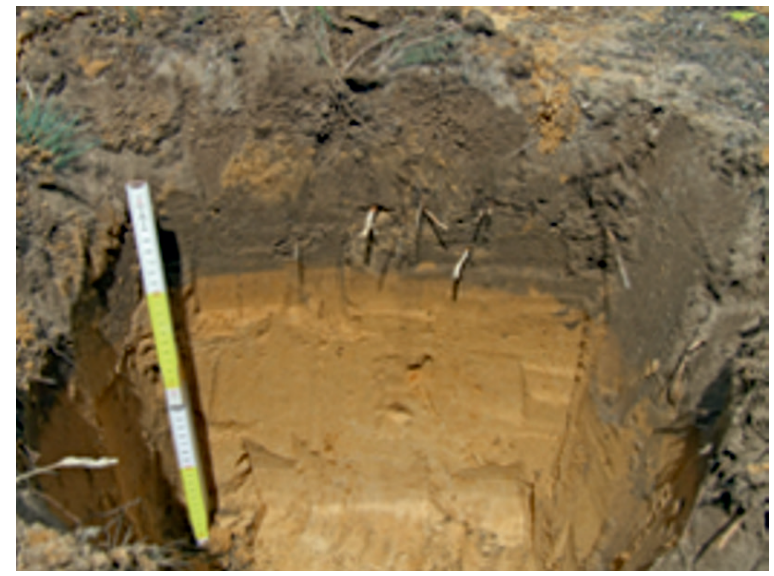

Depth Profile description

0-20 cm - humus, fine sand, brown and grey

$20-70 \mathrm{~cm}-$ medium and fine sand, yellow

70-110 cm - fine sand, silty, light yellow

Fig. 5.58. Soil pit in study plot Wola Makowska B (photo A. Majchrowska, 2013) 
Table 5.32. Study plot Wola Makowska B. Granulometric and chemical properties of soil

\begin{tabular}{|c|c|c|c|c|c|c|c|c|c|c|c|c|}
\hline \multirow[b]{2}{*}{ Soil pit } & \multirow{2}{*}{$\begin{array}{c}\text { Sampling } \\
\text { depth } \\
\text { cm }\end{array}$} & \multicolumn{9}{|c|}{$\begin{array}{c}\text { Grain size distribution } \\
\%\end{array}$} & \multirow{2}{*}{$\begin{array}{l}\text { Gra- } \\
\text { nulo- } \\
\text { metric } \\
\text { sub- } \\
\text { group }\end{array}$} & \multirow{2}{*}{$\begin{array}{c}\text { Spe- } \\
\text { cific } \\
\text { surface } \\
\text { area } \\
\mathrm{m}^{2} \cdot \mathrm{g}^{-1}\end{array}$} \\
\hline & & $\begin{array}{l}2.0- \\
1.0 \\
\mathrm{~mm}\end{array}$ & $\begin{array}{c}1.0- \\
0.5 \\
\mathrm{~mm}\end{array}$ & $\begin{array}{l}0.5- \\
0.25 \\
\mathrm{~mm}\end{array}$ & $\begin{array}{c}0.25- \\
0.1 \\
\mathrm{~mm}\end{array}$ & $\begin{array}{l}0.1- \\
0.05 \\
\mathrm{~mm}\end{array}$ & $\begin{array}{l}0.05- \\
0.02 \\
\mathrm{~mm}\end{array}$ & $\begin{array}{c}0.02- \\
0.005 \\
\mathrm{~mm}\end{array}$ & $\begin{array}{c}0.005- \\
0.002 \\
\mathrm{~mm}\end{array}$ & $\begin{array}{c}<0.002 \\
\mathrm{~mm}\end{array}$ & & \\
\hline $\begin{array}{l}\text { Wola Ma- } \\
\text { kowska B }\end{array}$ & $0-20$ & 0.2 & 19.4 & 43.4 & 29.3 & 2.9 & 2.3 & 1.7 & 0.8 & 0.1 & $\mathrm{p}$ & 0.0543 \\
\hline $\begin{array}{l}\text { Wola Ma- } \\
\text { kowska B }\end{array}$ & $20-40$ & 0.0 & 18.5 & 45.6 & 32.5 & 2.3 & 1.1 & 0.0 & 0.0 & 0.0 & $\mathrm{p}$ & 0.0245 \\
\hline $\begin{array}{l}\text { Wola Ma- } \\
\text { kowska B }\end{array}$ & $90-110$ & 0.0 & 9.7 & 38.4 & 38.9 & 6.4 & 3.1 & 2.0 & 0.9 & 0.6 & $\mathrm{p}$ & 0.0948 \\
\hline
\end{tabular}

\begin{tabular}{|c|c|c|c|c|c|c|c|c|c|c|}
\hline \multirow[t]{2}{*}{ Soil pit } & \multirow{2}{*}{$\begin{array}{c}\text { Sampling } \\
\text { depth } \\
\mathrm{cm}\end{array}$} & \multirow{2}{*}{$\begin{array}{c}\text { Humus } \\
\%\end{array}$} & \multirow{2}{*}{$\begin{array}{c}\mathrm{C} \text { total } \\
\%\end{array}$} & \multirow{2}{*}{$\begin{array}{l}\mathrm{N} \text { total } \\
\%\end{array}$} & \multirow[t]{2}{*}{$\mathrm{C} / \mathrm{N}$} & \multicolumn{2}{|c|}{$\begin{array}{l}\mathrm{pH} \\
\text { in }\end{array}$} & \multicolumn{3}{|c|}{$\begin{array}{l}\text { Available nutrients } \\
\mathrm{mg} / 100 \mathrm{~g}\end{array}$} \\
\hline & & & & & & $\mathrm{KCl}$ & $\mathrm{H}_{2} \mathrm{O}$ & $\mathrm{P}_{2} \mathrm{O}_{5}$ & $\mathrm{~K}_{2} \mathrm{O}$ & $\mathrm{Mg}$ \\
\hline $\begin{array}{l}\text { Wola Ma- } \\
\text { kowska B }\end{array}$ & $0-20$ & 0.85 & 0.493 & 0.041 & 12.02 & 4.2 & 4.6 & 12.4 & 0.3 & 0.3 \\
\hline $\begin{array}{l}\text { Wola Ma- } \\
\text { kowska B }\end{array}$ & $20-40$ & - & - & - & - & 4.6 & 4.8 & 7.1 & 0.3 & 0.3 \\
\hline $\begin{array}{l}\text { Wola Ma- } \\
\text { kowska B }\end{array}$ & $90-110$ & - & - & - & - & 4.4 & 4.7 & 3.0 & 0.6 & 0.3 \\
\hline
\end{tabular}

\begin{tabular}{|c|c|c|c|c|c|c|c|c|c|c|}
\hline & \multirow{2}{*}{ Sompling } & \multirow{2}{*}{$\begin{array}{c}\text { Hydrolytic } \\
\text { depth } \\
\text { acidity } \\
\text { mmol/100g }\end{array}$} & \multicolumn{4}{|c|}{$\begin{array}{c}\text { Exchangeable cations } \\
\mathrm{me} / 100 \mathrm{~g}\end{array}$} & \multicolumn{4}{c|}{$\begin{array}{c}\text { Sorption capacity } \\
\mathrm{me} / 100 \mathrm{~g}\end{array}$} \\
\cline { 5 - 12 } & & $\mathrm{Ca}^{2+}$ & $\mathrm{Mg}^{2+}$ & $\mathrm{Na}^{+}$ & $\mathrm{K}^{+}$ & $\mathrm{S}$ & $\mathrm{T}$ & $(\mathrm{T}-\mathrm{S})$ & $\mathrm{V}, \%$ \\
\hline $\begin{array}{c}\text { Wola Ma- } \\
\text { kowska B }\end{array}$ & $0-20$ & 2.70 & 0.10 & 0.017 & 0.017 & 0.031 & 0.165 & 2.865 & 2.70 & 5.76 \\
\hline $\begin{array}{c}\text { Wola Ma- } \\
\text { kowska B }\end{array}$ & $20-40$ & 1.48 & 0.15 & 0.017 & 0.009 & 0.026 & 0.202 & 1.682 & 1.48 & 12.01 \\
\hline $\begin{array}{c}\text { Wola Ma- } \\
\text { kowska B }\end{array}$ & $90-110$ & 1.30 & 0.10 & 0.015 & 0.009 & 0.026 & 0.150 & 1.450 & 1.30 & 10.34 \\
\hline
\end{tabular}

Source: own elaboration.

\section{Characteristics of the flora and fungi - Wola Makowska B}

Approximately $70 \%$ of the study plot is covered with plants. Poor in plant species (8). Two species dominate: Corynephorus canescens (45\%) and Hieracium pilosella (10\%). The share of other species oscillates between $5 \%$ and $0.5 \%$ of area coverage. The other identified plant species include: Anthoxanthum aristatum. Scarce specimens of
Pinus sylvestris are found in the plot. Among herbaceous plants, there are patches of moss - Bryum sp. and lichens - Cladonia furcata (Tab. 6.1).

Fungi of the plot are very sparse. They are represented by only 2 species of macromycetes. They are: Marasmius oreades and Conocybe rickeaniana (Tab. 7.1). 


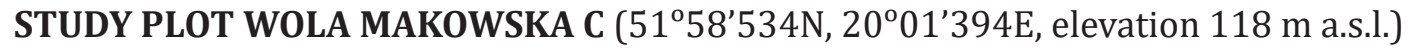
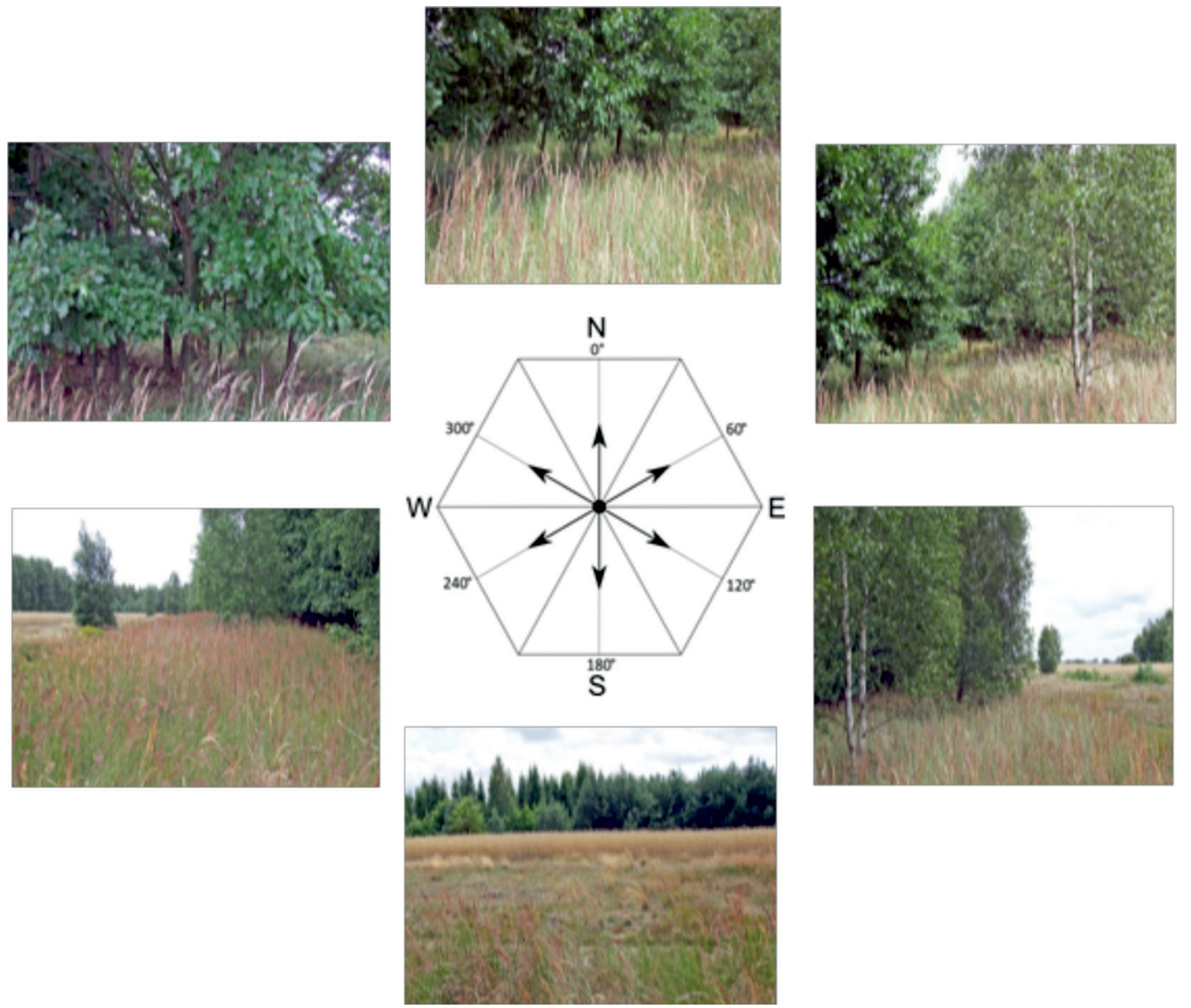

Fig. 5.59. Study plot Wola Makowska C (photo A. Majchrowska, 2013)

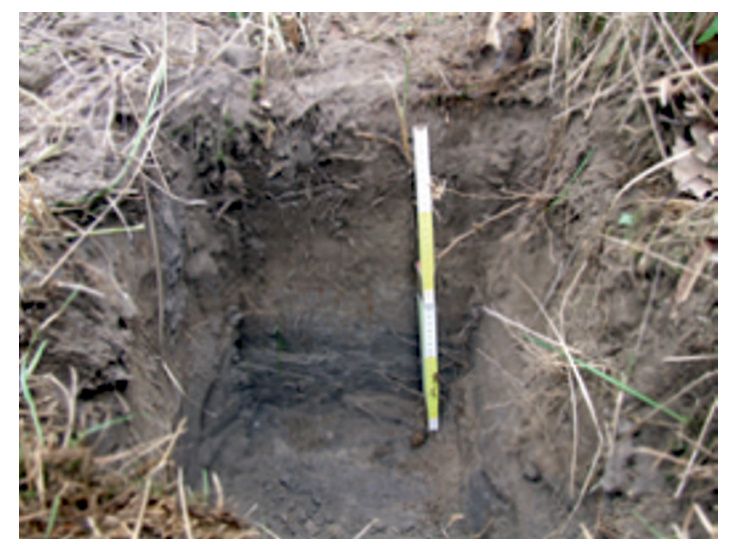

\section{Depth Profile description}

0-24 cm - humus, numerous grass roots, sandy, brown and grey

24-32 cm - humus, fine sand, silty, light brown and grey

32-60 cm - fine sand, dark grey

$60-90 \mathrm{~cm} \mathrm{-} \mathrm{fine} \mathrm{sand,} \mathrm{yellow} \mathrm{and} \mathrm{grey}$

90-120 cm - medium sand, grey, yellow and beige

Fig. 5.60. Soil pit in study plot Wola Makowska C (photo A. Majchrowska, 2013) 
Table 5.33. Study plot Wola Makowska C. Granulometric and chemical properties of soil

\begin{tabular}{|c|c|c|c|c|c|c|c|c|c|c|c|c|}
\hline \multirow[b]{2}{*}{ Soil pit } & \multirow{2}{*}{$\begin{array}{c}\text { Sampling } \\
\text { depth } \\
\mathrm{cm}\end{array}$} & \multicolumn{9}{|c|}{$\begin{array}{c}\text { Grain size distribution } \\
\%\end{array}$} & \multirow{2}{*}{$\begin{array}{l}\text { Gra- } \\
\text { nulo- } \\
\text { metric } \\
\text { sub- } \\
\text { group }\end{array}$} & \multirow{2}{*}{$\begin{array}{c}\text { Spe- } \\
\text { cific } \\
\text { surface } \\
\text { area } \\
\mathrm{m}^{2} \cdot \mathrm{g}^{-1}\end{array}$} \\
\hline & & $\begin{array}{l}2.0- \\
1.0 \\
\mathrm{~mm}\end{array}$ & $\begin{array}{l}1.0- \\
0.5 \\
\mathrm{~mm}\end{array}$ & $\begin{array}{l}0.5- \\
0.25 \\
\mathrm{~mm}\end{array}$ & $\begin{array}{c}0.25- \\
0.1 \\
\mathrm{~mm}\end{array}$ & $\begin{array}{l}0.1- \\
0.05 \\
\mathrm{~mm}\end{array}$ & $\begin{array}{l}0.05- \\
0.02 \\
\mathrm{~mm}\end{array}$ & $\begin{array}{c}0.02- \\
0.005 \\
\mathrm{~mm}\end{array}$ & $\begin{array}{c}0.005- \\
0.002 \\
\mathrm{~mm}\end{array}$ & $\begin{array}{c}<0.002 \\
\mathrm{~mm}\end{array}$ & & \\
\hline $\begin{array}{l}\text { Wola Ma- } \\
\text { kowska C }\end{array}$ & $0-20$ & 0 & 12.8 & 37.8 & 31.6 & 7.8 & 6.1 & 2.9 & 0.8 & 0.2 & $\mathrm{p}$ & 0.0747 \\
\hline $\begin{array}{l}\text { Wola Ma- } \\
\text { kowska C }\end{array}$ & $20-40$ & 0 & 13.0 & 38.5 & 29.8 & 6.0 & 6.0 & 4.6 & 1.3 & 0.7 & $\mathrm{p}$ & 0.1230 \\
\hline $\begin{array}{l}\text { Wola Ma- } \\
\text { kowska C }\end{array}$ & $90-110$ & 0 & 14.6 & 53.5 & 31.9 & 0.0 & 0.0 & 0.0 & 0.0 & 0.0 & $\mathrm{p}$ & 0.0213 \\
\hline
\end{tabular}

\begin{tabular}{|c|c|c|c|c|c|c|c|c|c|c|}
\hline \multirow[t]{2}{*}{ Soil pit } & \multirow{2}{*}{$\begin{array}{c}\text { Sampling } \\
\text { depth } \\
\mathrm{cm}\end{array}$} & \multirow{2}{*}{$\begin{array}{c}\text { Humus } \\
\%\end{array}$} & \multirow{2}{*}{$\begin{array}{c}\text { C total } \\
\%\end{array}$} & \multirow{2}{*}{$\begin{array}{c}\mathrm{N} \text { total } \\
\%\end{array}$} & \multirow[t]{2}{*}{$\mathrm{C} / \mathrm{N}$} & \multicolumn{2}{|c|}{$\begin{array}{l}\mathrm{pH} \\
\text { in }\end{array}$} & \multicolumn{3}{|c|}{$\begin{array}{c}\text { Available nutrients } \\
\mathrm{mg} / 100 \mathrm{~g}\end{array}$} \\
\hline & & & & & & $\mathrm{KCl}$ & $\mathrm{H}_{2} \mathrm{O}$ & $\mathrm{P}_{2} \mathrm{O}_{5}$ & $\mathrm{~K}_{2} \mathrm{O}$ & $\mathrm{Mg}$ \\
\hline $\begin{array}{l}\text { Wola Ma- } \\
\text { kowska C }\end{array}$ & $0-20$ & 1.70 & 0.986 & 0.078 & 12.64 & 4.1 & 4.5 & 5.0 & 0.6 & 0.4 \\
\hline $\begin{array}{l}\text { Wola Ma- } \\
\text { kowska C }\end{array}$ & $20-40$ & - & - & - & - & 4.3 & 4.8 & 1.7 & 0.4 & 0.3 \\
\hline $\begin{array}{l}\text { Wola Ma- } \\
\text { kowska C }\end{array}$ & $90-110$ & - & - & - & - & 4.6 & 5.2 & 0.9 & 0.4 & 0.3 \\
\hline
\end{tabular}

\begin{tabular}{|c|c|c|c|c|c|c|c|c|c|c|}
\hline & \multirow{2}{*}{ Sampling } & \multirow{2}{*}{$\begin{array}{c}\text { Hydrolytic } \\
\text { depth } \\
\text { cmidity } \\
\text { mmol/100g }\end{array}$} & \multicolumn{4}{|c|}{$\begin{array}{c}\text { Exchangeable cations } \\
\text { me/100g }\end{array}$} & \multicolumn{4}{c|}{$\begin{array}{c}\text { Sorption capacity } \\
\text { me/100g }\end{array}$} \\
\hline $\begin{array}{c}\text { Wola Ma- } \\
\text { kowska C }\end{array}$ & $0-20$ & 4.72 & 0.15 & 0.027 & 0.004 & 0.031 & 0.212 & 4.932 & 4.72 & 4.30 \\
\hline $\begin{array}{c}\text { Wola Ma- } \\
\text { kowska C }\end{array}$ & $20-40$ & 4.88 & 0.15 & 0.020 & 0.017 & 0.026 & 0.213 & 5.093 & 4.88 & 4.18 \\
\hline $\begin{array}{c}\text { Wola Ma- } \\
\text { kowska C }\end{array}$ & $90-110$ & 0.94 & 0.10 & 0.018 & 0.009 & 0.018 & 0.145 & 1.085 & 0.94 & 13.36 \\
\hline
\end{tabular}

Source: own elaboration.

\section{Characteristics of the flora and fungi - Wola Makowska C}

Approximately $90 \%$ of the study plot is covered with plants. Very poor in plant species (7). One species dominates: Calamagrostis epigeios (85\%). Other plants include only Agrostos capillaris, which covers about $5 \%$ of the area. The other species show only a slight coverage. They include:
Hieracium pilosella, Betula pendula, Epilobium angustifolium and Quercus rubra (Tab. 6.1).

Fungi of the plot are very sparse. They are represented by only 2 species of macromycetes. They are: Marasmius oreades and Psilocybe semilanceata (Tab. 7.1).

\section{Analysis of granulometric composition and chemical properties of soils in the Wola Makowska study plot group}

On the basis of the results of grain size distribution analyses (Tab. 5.31-5.33) at all study plots and soil horizons, the granulometric group of sand (p) was identified (Polskie Towarzystwo Gleboznawcze 2009). The share of sandy fraction ranged from $87.3 \%$ to $100 \%$, and the total content of the fraction below $0.02 \mathrm{~mm}$ fell within the range $0-6.6 \%$ and was at its highest in the horizon of $20-40 \mathrm{~cm}$, at plot Wola Makowska C. On the basis of the granulometric composition, the soils 
in the Wola Makowska study plot group were classified as very light in the category of agrotechnical heaviness.

At plot Wola Makowska C, in the horizon of 20-40 cm, the specific surface area reached the maximum value of only $0.123 \mathrm{~m}^{2} \cdot \mathrm{g}^{-1}$, and in general this index was very low at all analysed soil horizons, due to the low content of the clayey and silty fractions.

The humus content ranged from $0.63 \%$ at plot A to $1.70 \%$ at plot $\mathrm{C}$. The lowest value occurred in leeched brown soil, formed upon aeolian sands, and the highest - at the plot located on the border between the leeched brown and mucky soil types. The $\mathrm{C} / \mathrm{N}$ ration was narrow and amounted to about 12:1-11:1.

The $\mathrm{pH}$ in $1 \mathrm{M} \mathrm{KCl}$ in all soil horizons indicated very acidic reaction and only in horizons 20-40 $\mathrm{cm}$ at plot Wola Makowska B and $90-110 \mathrm{~cm}$ at plot Wola Makowska C, the reaction was acidic.

The surface horizon of soils at plots Wola Makowska A and B was characterised by medium con- tent of available phosphorus, whereas deeper horizons showed medium, low or very low content of this element. The soil at plot $C$ was very poor in available forms of phosphorus along the entire profile. The content of available potassium and magnesium in all analysed soil horizons was very low.

The soils were characterised by varied hydrolytic acidity, with values within the range from 0.94 to $4.88 \mathrm{mmol} / 100 \mathrm{~g}$. The total content of exchangeable alkaline cations was low, as it was ranging from $0.145 \mathrm{mmol} / 100 \mathrm{~g}$ to $0.625 \mathrm{mmol} / 100 \mathrm{~g}$, and its most important component was calcium. Total cation exchange capacity was very low: the maximum value was $5.093 \mathrm{mmol} / 100 \mathrm{~g}$. Saturation of the sorptive complex with alkaline ions (V) was very low $(4.18 \%-13.36 \%)$ at plots Wola Makowska B and C. At plot Wola Makowska A, in the horizon of $90-110 \mathrm{~cm}$, the degree of saturation with alkalis was the highest, though it was low in general, amounting to $28.32 \%$.

\section{SZYMANISZKI STUDY PLOT GROUP}

The Szymaniszki study plot group is located about $750 \mathrm{~m}$ from the borders of the Łódź Hills Landscape Park, on the north-eastern outskirts of Brzeziny. According to the physicogeographical regionalisation of Poland (Kondracki 2002), it lies in the mesoregion of the Łódź Hills, within the macroregion of the South Mazovian Hills.

The analysed area encompasses alternating belts of farmlands, abandoned fields and narrow strips of woodland. In the south, it is restricted by buildings of Szymaniszki. In the east, it borders arable lands, with single strips of abandoned lands and a group of farm buildings and several recreational lots. To the south, there are mainly arable lands. To the west, about 200 metres away, there is a developing pit of a sand mine.

The geological substratum of the area consists of deposits from the Warta Glaciation, diversified genetically and lithologically (Trzmiel 1990). In the south-western part (study plot Szymaniszki A), there are sands and gravels with pebbles of end moraines as well as glacial sands and gravels; in the northern part (study plot Szymaniszki B) glacial tills, whereas in the remaining area (study plot Szymaniszki C) - fluvioglacial sands.

On the basis of a soil and agricultural map (http://geoportal.lodzkie.pl/imap/), at plot Szymaniszki A leeched brown soil was identified, formed from loamy and silty sand, underneath which lies slightly loamy sand and loose sand. The soil was classified as complex 6 of agricultural suitability - poor rye and class $\mathrm{V}$ of arable land valuation. At plots Szymaniszki B and C, there are leeched brown soils, formed from silts, lying over loamy sands. The soils were classified as complex 5 of agricultural suitability - good rye and valuation class IVb. The soil and agricultural map shows the presence of silty and sandy deposits which, due to their low thickness - about $0.5 \mathrm{~m}$ - were omitted in the geological map.

The diverse geological substratum is reflected in the complex structure of natural environment of the Szymaniszki group, as each of the three study plots was classified as a different type of morpholithohydrotope: Szymaniszki A - lies in a type 4 geocomplex - boulders, cobbles, gravels, sands and muds of moraine hills, kame hills and esker ridges; Szymaniszki B - in a type 5 geocomplex glacial tills of plateaus; Szymaniszki C - in a type 6 geocomplex - glacial and fluvioglacial boulders, cobbles, gravels, sands and muds of plateaus, alluvial fans and erosional-accumulational terraces.

Photographic documentation of the Szymaniszki study plot group and analysis results of granulometric composition and chemical properties of soils are presented in figures 5.61-5.66 and in tables 5.34-5.36. 
STUDY PLOT SZYMANISZKI A (51º8'944N, 19046'556E, elevation 229 m a.s.l.)
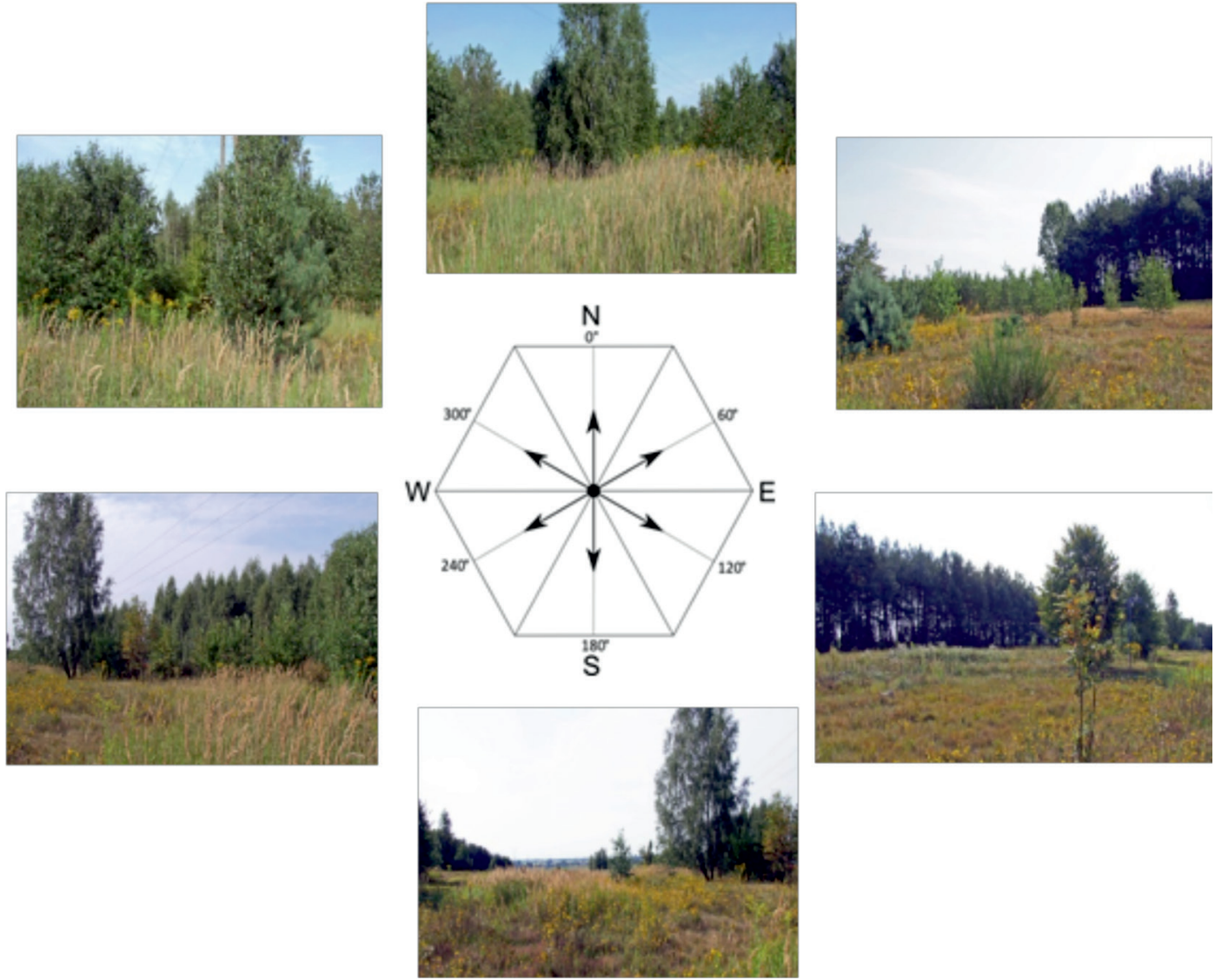

Fig. 5.61. Study plot Szymaniszki A (photo A. Majchrowska, 2013)

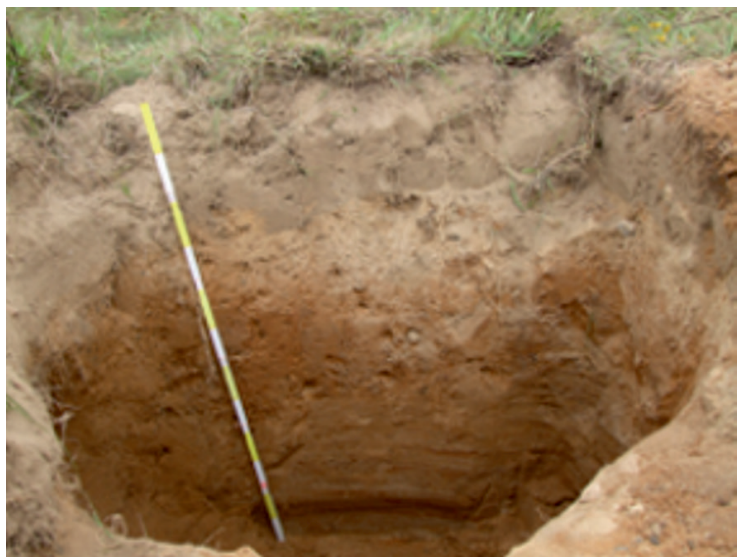

Depth Profile description

$0-30 \mathrm{~cm} \mathrm{-} \mathrm{humus,} \mathrm{sandy} \mathrm{and} \mathrm{silty,} \mathrm{grey} \mathrm{and} \mathrm{beige}$

$30-45 \mathrm{~cm} \mathrm{-} \mathrm{sand} \mathrm{with} \mathrm{gravel} \mathrm{and} \mathrm{silt,} \mathrm{grey,} \mathrm{beige} \mathrm{and}$ yellow

45-70 cm - unsorted and coarse sand, with gravel and cobbles, rusty

$70-110 \mathrm{~cm}$ - coarse sand, yellow and rusty; with layers of gravel

110-150 cm - coarse sand, with increasing content of gravel, yellow and rusty, moist

Fig. 5.62. Soil pit in study plot Szymaniszki A (photo. A. Majchrowska, 2013) 
Table 5.34. Study plot Szymaniszki A. Granulometric and chemical properties of soil

\begin{tabular}{|c|c|c|c|c|c|c|c|c|c|c|c|c|}
\hline \multirow[b]{2}{*}{ Soil pit } & \multirow{2}{*}{$\begin{array}{c}\text { Sam- } \\
\text { pling } \\
\text { depth } \\
\text { cm }\end{array}$} & \multicolumn{9}{|c|}{$\begin{array}{c}\text { Grain size distribution } \\
\%\end{array}$} & \multirow{2}{*}{$\begin{array}{l}\text { Gra- } \\
\text { nulo- } \\
\text { metric } \\
\text { sub- } \\
\text { group }\end{array}$} & \multirow{2}{*}{$\begin{array}{c}\text { Spe- } \\
\text { cific } \\
\text { surfa- } \\
\text { ce area } \\
\mathrm{m}^{2} \cdot \mathrm{g}^{-1}\end{array}$} \\
\hline & & $\begin{array}{c}2.0- \\
1.0 \\
\mathrm{~mm}\end{array}$ & $\begin{array}{c}1.0- \\
0.5 \\
\mathrm{~mm}\end{array}$ & $\begin{array}{c}0.5- \\
0.25 \\
\mathrm{~mm}\end{array}$ & $\begin{array}{c}0.25- \\
0.1 \\
\mathrm{~mm}\end{array}$ & $\begin{array}{c}0.1- \\
0.05 \\
\mathrm{~mm}\end{array}$ & $\begin{array}{l}0.05- \\
0.02 \\
\mathrm{~mm}\end{array}$ & $\begin{array}{c}0.02- \\
0.005 \\
\mathrm{~mm}\end{array}$ & $\begin{array}{c}0.005- \\
0.002 \\
\mathrm{~mm}\end{array}$ & $\begin{array}{c}<0.002 \\
\mathrm{~mm}\end{array}$ & & \\
\hline Szymaniszki A & $0-20$ & 5.5 & 29.9 & 24.6 & 6.6 & 9.5 & 14.0 & 6.7 & 1.7 & 1.5 & $\mathrm{pg}$ & 0.2110 \\
\hline Szymaniszki A & $20-40$ & 6.0 & 38.2 & 33.0 & 5.3 & 3.9 & 6.5 & 4.6 & 1.4 & 1.1 & ps & 0.1460 \\
\hline Szymaniszki A & $90-110$ & 6.7 & 49.8 & 39.3 & 4.2 & 0.0 & 0.0 & 0.0 & 0.0 & 0.0 & $\mathrm{p}$ & 0.0124 \\
\hline
\end{tabular}

\begin{tabular}{|c|c|c|c|c|c|c|c|c|c|c|}
\hline \multirow{2}{*}{ Soil pit } & \multirow{2}{*}{$\begin{array}{l}\text { Sam- } \\
\text { pling } \\
\text { depth } \\
\mathrm{cm}\end{array}$} & \multirow{2}{*}{$\begin{array}{c}\text { Humus } \\
\%\end{array}$} & \multirow{2}{*}{$\begin{array}{c}\text { C total } \\
\%\end{array}$} & \multirow{2}{*}{$\begin{array}{c}\mathrm{N} \text { total } \\
\%\end{array}$} & \multirow{2}{*}{$\mathrm{C} / \mathrm{N}$} & \multicolumn{2}{|c|}{$\begin{array}{l}\mathrm{pH} \\
\text { in }\end{array}$} & \multicolumn{3}{|c|}{$\begin{array}{c}\text { Available nutrients } \\
\mathrm{mg} / 100 \mathrm{~g}\end{array}$} \\
\hline & & & & & & $\mathrm{KCl}$ & $\mathrm{H}_{2} \mathrm{O}$ & $\mathrm{P}_{2} \mathrm{O}_{5}$ & $\mathrm{~K}_{2} \mathrm{O}$ & $\mathrm{Mg}$ \\
\hline Szymaniszki A & $0-20$ & 1.24 & 0.719 & 0.069 & 10.42 & 4.3 & 5.1 & 6.2 & 5.6 & 0.6 \\
\hline Szymaniszki A & $20-40$ & - & - & - & - & 4.4 & 5.2 & 2.9 & 3.4 & 0.7 \\
\hline Szymaniszki A & $90-110$ & - & - & - & - & 4.8 & 6.1 & 2.1 & 2.1 & 1.0 \\
\hline
\end{tabular}

\begin{tabular}{|c|c|c|c|c|c|c|c|c|c|c|}
\hline \multirow{2}{*}{ Soil pit } & \multirow{2}{*}{$\begin{array}{l}\text { Sam- } \\
\text { pling } \\
\text { depth } \\
\text { cm }\end{array}$} & \multirow{2}{*}{$\begin{array}{l}\text { Hydrolytic } \\
\text { acidity } \\
\text { mmol/100g }\end{array}$} & \multicolumn{4}{|c|}{$\begin{array}{c}\text { Exchangeable cations } \\
\text { me } / 100 \mathrm{~g}\end{array}$} & \multicolumn{4}{|c|}{$\begin{array}{c}\text { Sorption capacity } \\
\text { me } / 100 \mathrm{~g}\end{array}$} \\
\hline & & & $\mathrm{Ca}^{2+}$ & $\mathrm{Mg}^{2+}$ & $\mathrm{Na}^{+}$ & $\mathrm{K}^{+}$ & S & $\mathrm{T}$ & $(\mathrm{T}-\mathrm{S})$ & V, \% \\
\hline Szymaniszki A & $0-20$ & 3.94 & 0.70 & 0.050 & 0.017 & 0.143 & 0.910 & 4.850 & 3.94 & 18.76 \\
\hline Szymaniszki A & $20-40$ & 1.80 & 0.80 & 0.053 & 0.017 & 0.072 & 0.942 & 2.742 & 1.80 & 34.35 \\
\hline Szymaniszki A & $90-110$ & 0.49 & 0.70 & 0.078 & 0.017 & 0.036 & 0.831 & 1.321 & 0.49 & 62.91 \\
\hline
\end{tabular}

Source: own elaboration.

\section{Characteristics of the flora and fungi - Szymaniszki A}

Approximately $100 \%$ of the study plot is covered with plants. Fairly rich in plant species (17). Four species dominate: Hieracium umbellatum (30\%), Calamagrostis epigeios (25\%), Agrostis capillaris $(15 \%)$ and Thymus pulegioides $(10 \%)$. The share of other species ranges from $5 \%$ to $0.5 \%$ of area coverage. The other identified species of plants include: Convolvulus arvensis, Holcus mollis, Hypericum perforatum, Gnaphalium sylvaticum (Tab. 6.1).
The plot features specimens of trees and shrubs: Pinus sylvestris, Betula pendula and Padus serotina.

Fungi of the plot are not abundant. They are represented by 8 species of macromycetes. The most frequently found species include: Bovista plumbea and Amanita muscaria (Tab. 7.1). 
STUDY PLOT SZYMANISZKI B (51ํ49'089N, 19²46'598E, elevation 214 m a.s.l.)
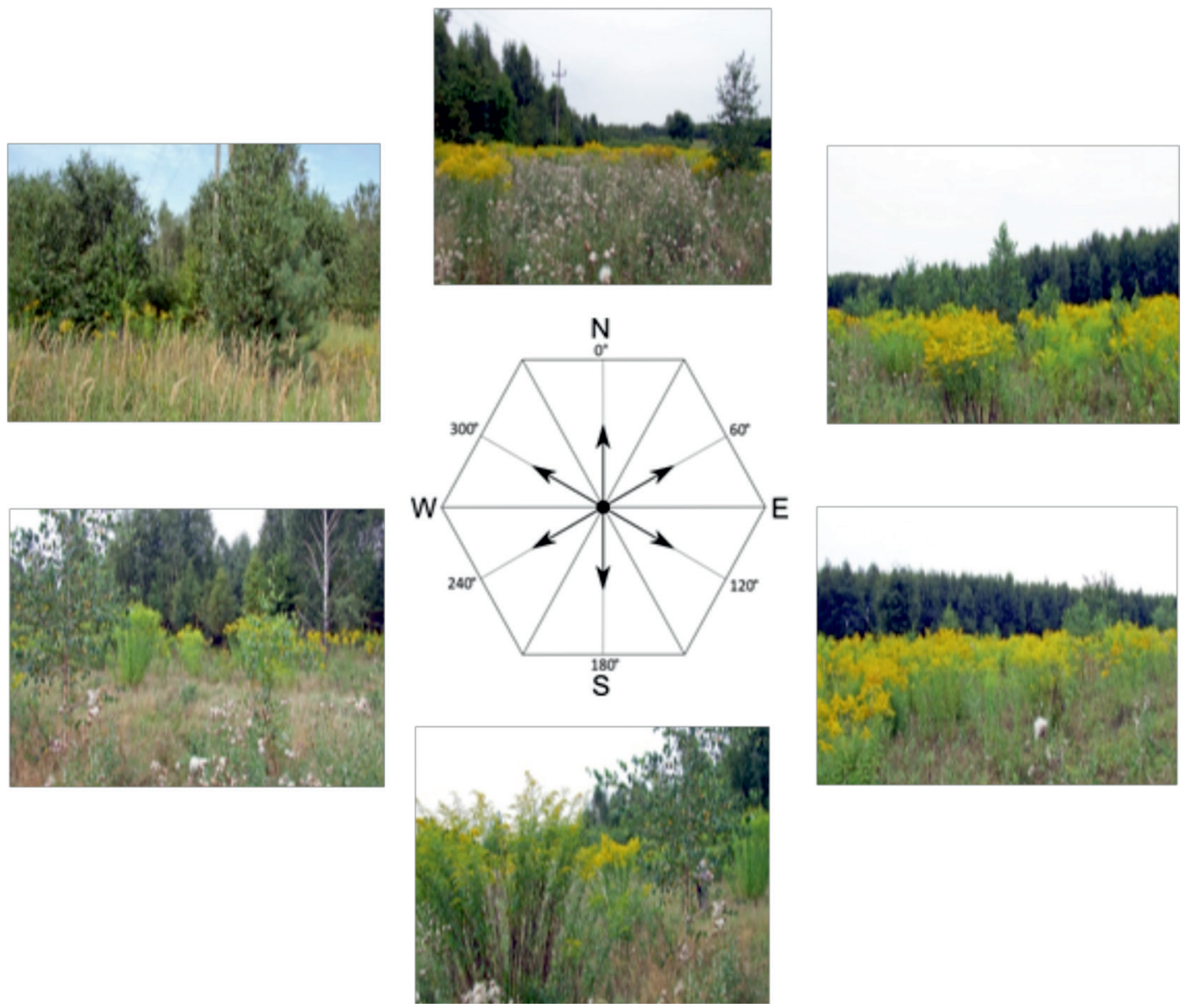

Fig. 5.63. Study plot Szymaniszki B (photo A. Majchrowska, 2013)

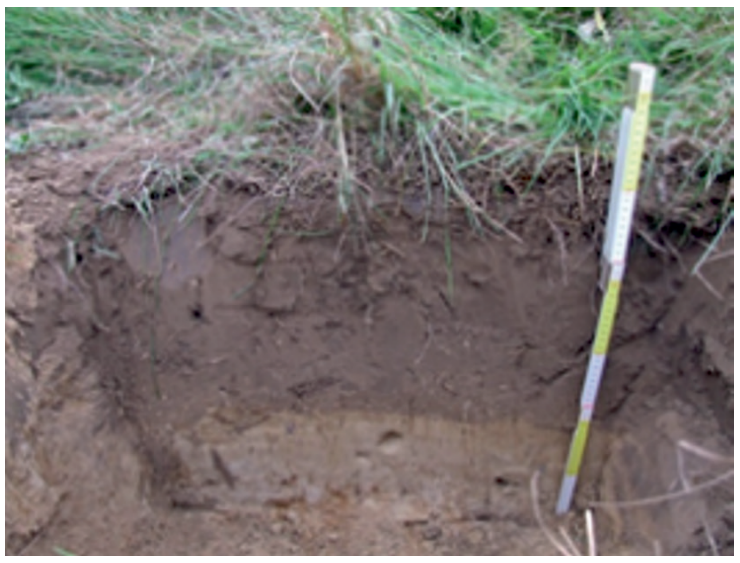

$$
\begin{aligned}
& \text { Depth } \text { Profile description } \\
& 0-32 \mathrm{~cm}- \text { humus, silty and clayey, grey and brown } \\
& 32-70 \mathrm{~cm}- \text { yellow and beige silt, with single cobbles } \\
& \text { of } 5 \mathrm{~cm} \text { in diameter } \\
& 70-80 \mathrm{~cm}- \text { yellow and beige fine sand, with gravel } \\
& \text { and cobbles } \\
& 80-130 \mathrm{~cm}- \text { light beige silty sand, alternating with } \\
& \text { strata of coarse sand, red, with gravel } \\
& 130-140 \mathrm{~cm}-\begin{array}{l}
\text { coarse sand, with gravel, somewhat } \\
\text { loamy, rusty }
\end{array}
\end{aligned}
$$

Fig. 5.64. Soil pit in study plot Szymaniszki B (photo. A. Majchrowska, 2013) 
Table 5.35. Study plot Szymaniszki B. Granulometric and chemical properties of soil

\begin{tabular}{|c|c|c|c|c|c|c|c|c|c|c|c|c|}
\hline \multirow[b]{2}{*}{ Soil pit } & \multirow{2}{*}{$\begin{array}{c}\text { Sampling } \\
\text { depth } \\
\text { cm }\end{array}$} & \multicolumn{9}{|c|}{$\begin{array}{c}\text { Grain size distribution } \\
\%\end{array}$} & \multirow{2}{*}{$\begin{array}{l}\text { Gra- } \\
\text { nulo- } \\
\text { metric } \\
\text { sub- } \\
\text { group }\end{array}$} & \multirow{2}{*}{$\begin{array}{c}\text { Spe- } \\
\text { cific } \\
\text { surfa- } \\
\text { ce area } \\
\mathrm{m}^{2} \cdot \mathrm{g}^{-1}\end{array}$} \\
\hline & & $\begin{array}{l}2.0- \\
1.0 \\
\mathrm{~mm}\end{array}$ & $\begin{array}{l}1.0- \\
0.5 \\
\mathrm{~mm}\end{array}$ & $\begin{array}{l}0.5- \\
0.25 \\
\mathrm{~mm}\end{array}$ & $\begin{array}{c}0.25- \\
0.1 \\
\mathrm{~mm}\end{array}$ & $\begin{array}{l}0.1- \\
0.05 \\
\mathrm{~mm}\end{array}$ & $\begin{array}{l}0.05- \\
0.02 \\
\mathrm{~mm}\end{array}$ & $\begin{array}{c}0.02- \\
0.005 \\
\mathrm{~mm}\end{array}$ & $\begin{array}{c}0.005- \\
0.002 \\
\mathrm{~mm}\end{array}$ & $\begin{array}{c}<0.002 \\
\mathrm{~mm}\end{array}$ & & \\
\hline Szymaniszki B & $0-20$ & 0.5 & 11.6 & 19.4 & 13.0 & 16.7 & 23.2 & 10.9 & 2.4 & 2.3 & gp & 0.323 \\
\hline Szymaniszki B & $20-40$ & 1.3 & 10.6 & 16.0 & 10.3 & 16.6 & 25.7 & 13.2 & 3.3 & 3.0 & gp & 0.402 \\
\hline Szymaniszki B & $90-110$ & 2.6 & 32.2 & 42.4 & 16.0 & 1.20 & 1.90 & 2.40 & 1.0 & 0.3 & $\mathrm{p}$ & 0.065 \\
\hline
\end{tabular}

\begin{tabular}{|c|c|c|c|c|c|c|c|c|c|c|}
\hline \multirow{2}{*}{ Soil pit } & \multirow{2}{*}{$\begin{array}{c}\text { Sampling } \\
\text { depth } \\
\mathrm{cm}\end{array}$} & $\begin{array}{c}\text { Humus } \\
\%\end{array}$ & \multirow{2}{*}{$\begin{array}{c}\mathrm{C} \text { total } \\
\%\end{array}$} & \multirow{2}{*}{$\begin{array}{c}\mathrm{N} \text { total } \\
\%\end{array}$} & $\mathrm{C} / \mathrm{N}$ & \multicolumn{2}{|c|}{$\begin{array}{c}\mathrm{pH} \\
\text { in }\end{array}$} & \multicolumn{3}{|c|}{ Available nutrients } \\
\cline { 6 - 11 } & & & & & $\mathrm{KCl}$ & $\mathrm{H}_{2} \mathrm{O}$ & $\mathrm{P}_{2} \mathrm{O}_{5}$ & $\mathrm{~K}_{2} \mathrm{O}$ & $\mathrm{Mg}$ \\
\hline Szymaniszki B & $0-20$ & 1.86 & 1.079 & 0.100 & 10.788 & 4.4 & 5.1 & 5.3 & 3.8 & 1.6 \\
\hline Szymaniszki B & $20-40$ & - & - & - & - & 4.6 & 5.4 & 4.5 & 2.3 & 1.1 \\
\hline Szymaniszki B & $90-110$ & - & - & - & - & 5.1 & 6.5 & 3.5 & 1.7 & 2.8 \\
\hline
\end{tabular}

\begin{tabular}{|c|c|c|c|c|c|c|c|c|c|c|}
\hline \multirow{2}{*}{ Soil pit } & \multirow{2}{*}{$\begin{array}{c}\text { Sampling } \\
\text { depth } \\
\text { cm }\end{array}$} & \multirow{2}{*}{$\begin{array}{l}\text { Hydrolytic } \\
\text { acidity } \\
\mathrm{mmol} / 100 \mathrm{~g}\end{array}$} & \multicolumn{4}{|c|}{$\begin{array}{l}\text { Exchangeable cations } \\
\text { me/100g }\end{array}$} & \multicolumn{4}{|c|}{$\begin{array}{l}\text { Sorption capacity } \\
\text { me/100g }\end{array}$} \\
\hline & & & $\mathrm{Ca}^{2+}$ & $\mathrm{Mg}^{2+}$ & $\mathrm{Na}^{+}$ & $\mathrm{K}^{+}$ & S & $\mathrm{T}$ & (T-S) & V, \% \\
\hline Szymaniszki B & $0-20$ & 4.39 & 1.80 & 0.145 & 0.043 & 0.108 & 2.096 & 6.486 & 4.39 & 32.32 \\
\hline Szymaniszki B & $20-40$ & 3.14 & 1.20 & 0.088 & 0.035 & 0.072 & 1.395 & 4.535 & 3.14 & 30.76 \\
\hline Szymaniszki B & $90-110$ & 0.60 & 1.55 & 0.268 & 0.039 & 0.036 & 1.893 & 2.493 & 0.60 & 75.93 \\
\hline
\end{tabular}

Source: own elaboration.

\section{Characteristics of the flora and fungi - Szymaniszki B}

Approximately $100 \%$ of the study plot is covered with plants. Poor in species of plants (5). Two species dominate: Cirsium arvense (60\%), Solidago canadensis (30\%). The share of other species is about $0.5 \%$ of area coverage each. The other identified species of plants include: Hypericum perforatum, Poa pratensis and Betula pendula.
Fungi of the plot are sparse. They are represented by 3 species of macromycetes. The most frequently found species is Marasmius oreades. The undergrowth also features the following: Conocybe rickeaniana and Macrolepiota procera (Tab. 7.1). 
STUDY PLOT SZYMANISZKI C (5149'047N, 19²46'804E, elevation 220 m a.s.l.)
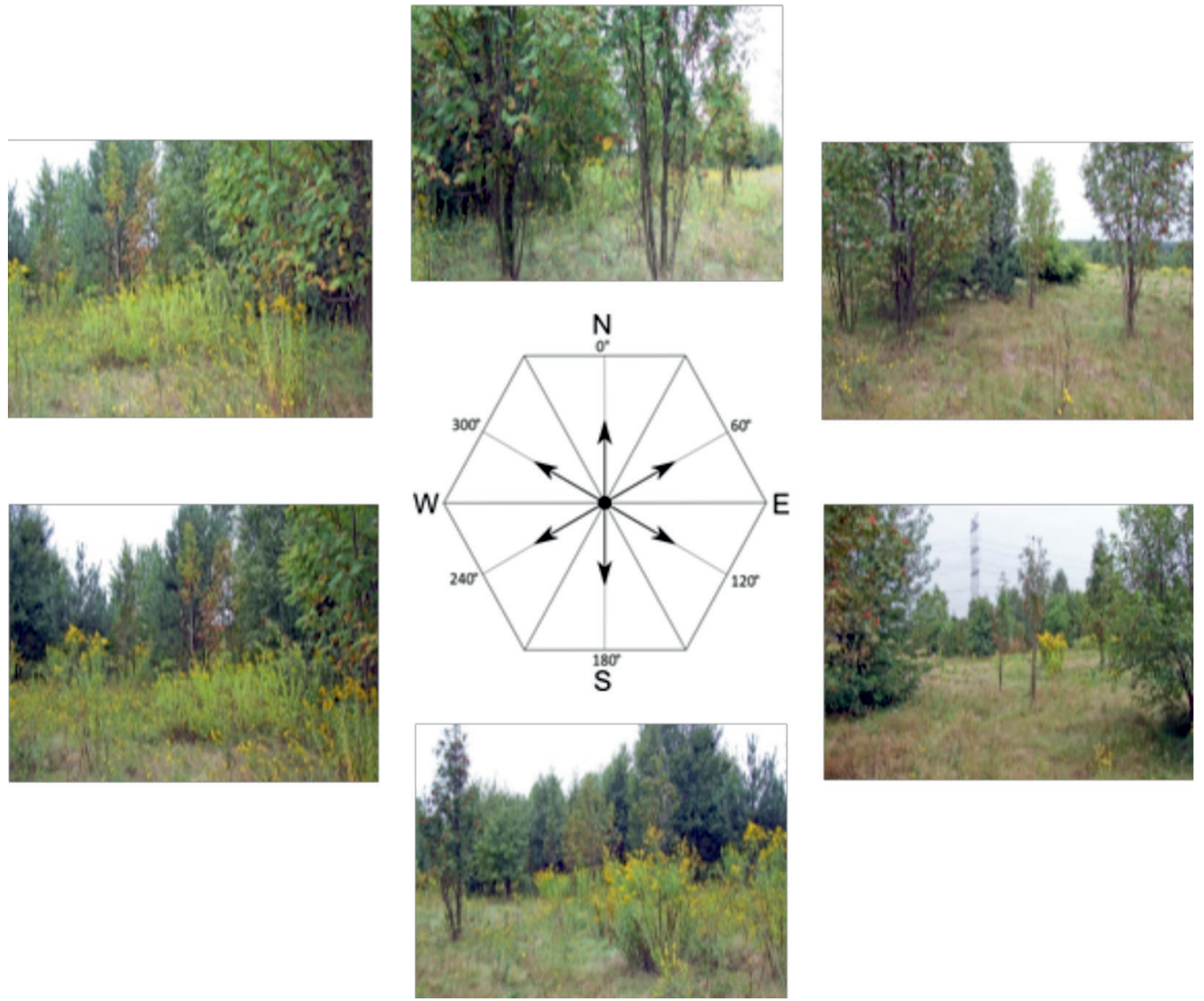

Fig. 5.65. Study plot Szymaniszki C (photo A. Majchrowska, 2013)

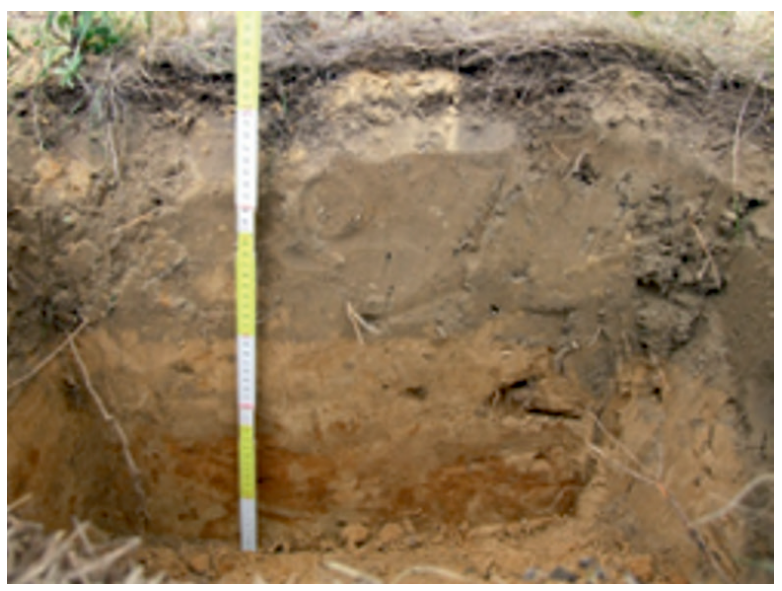

Depth Profile description

0-25 cm - humus, silty, dark brown and grey $25-40 \mathrm{~cm}$ - fine silty sand, golden

40-90 cm - fine and medium sand, silty, golden and rusty with rusty smudges

90-140 cm - medium and fine sand, silty, yellow

Fig. 5.66. Soil pit in study plot Szymaniszki C (photo. A. Majchrowska, 2013) 
Table 5.36. Study plot Szymaniszki C. Granulometric and chemical properties of soil

\begin{tabular}{|c|c|c|c|c|c|c|c|c|c|c|c|c|}
\hline \multirow[b]{2}{*}{ Soil pit } & \multirow{2}{*}{$\begin{array}{c}\text { Sam- } \\
\text { pling } \\
\text { depth } \\
\text { cm }\end{array}$} & \multicolumn{9}{|c|}{$\begin{array}{c}\text { Grain size distribution } \\
\% \\
\end{array}$} & \multirow{2}{*}{$\begin{array}{l}\text { Gra- } \\
\text { nulo- } \\
\text { metric } \\
\text { sub- } \\
\text { group }\end{array}$} & \multirow{2}{*}{$\begin{array}{c}\text { Spe- } \\
\text { cific } \\
\text { surfa- } \\
\text { ce area } \\
\mathrm{m}^{2} \cdot \mathrm{g}^{-1}\end{array}$} \\
\hline & & $\begin{array}{l}2.0- \\
1.0 \\
\mathrm{~mm} \\
\end{array}$ & $\begin{array}{l}1.0- \\
0.5 \\
\mathrm{~mm} \\
\end{array}$ & $\begin{array}{l}0.5- \\
0.25 \\
\mathrm{~mm}\end{array}$ & $\begin{array}{c}0.25- \\
0.1 \\
\mathrm{~mm}\end{array}$ & $\begin{array}{c}0.1- \\
0.05 \\
\mathrm{~mm} \\
\end{array}$ & $\begin{array}{l}0.05- \\
0.02 \\
\mathrm{~mm}\end{array}$ & $\begin{array}{c}0.02- \\
0.005 \\
\mathrm{~mm}\end{array}$ & $\begin{array}{c}0.005- \\
0.002 \\
\mathrm{~mm}\end{array}$ & $\begin{array}{c}<0.002 \\
\mathrm{~mm}\end{array}$ & & \\
\hline Szymaniszki C & $0-20$ & 0.1 & 7.4 & 17.6 & 17.7 & 18.3 & 23.5 & 10.7 & 2.5 & 2.3 & gp & 0.331 \\
\hline Szymaniszki C & $20-40$ & 0.0 & 2.5 & 19.0 & 46.7 & 17.6 & 6.2 & 4.8 & 1.8 & 1.3 & ps & 0.187 \\
\hline Szymaniszki C & $90-110$ & 0.0 & 7.1 & 27.7 & 44.3 & 14.5 & 3.1 & 1.8 & 0.9 & 0.6 & $\mathrm{p}$ & 0.101 \\
\hline
\end{tabular}

\begin{tabular}{|c|c|c|c|c|c|c|c|c|c|c|}
\hline \multirow{2}{*}{ Soil pit } & \multirow{2}{*}{$\begin{array}{l}\text { Sam- } \\
\text { pling } \\
\text { depth } \\
\text { cm }\end{array}$} & \multirow{2}{*}{$\begin{array}{c}\text { Humus } \\
\%\end{array}$} & \multirow{2}{*}{$\begin{array}{l}\mathrm{C} \text { total } \\
\%\end{array}$} & \multirow{2}{*}{$\begin{array}{l}\mathrm{N} \text { total } \\
\%\end{array}$} & \multirow{2}{*}{$\mathrm{C} / \mathrm{N}$} & \multicolumn{2}{|c|}{$\begin{array}{l}\mathrm{pH} \\
\text { in }\end{array}$} & \multicolumn{3}{|c|}{$\begin{array}{l}\text { Available nutrients } \\
\mathrm{mg} / 100 \mathrm{~g}\end{array}$} \\
\hline & & & & & & $\mathrm{KCl}$ & $\mathrm{H}_{2} \mathrm{O}$ & $\mathrm{P}_{2} \mathrm{O}_{5}$ & $\mathrm{~K}_{2} \mathrm{O}$ & $\mathrm{Mg}$ \\
\hline Szymaniszki C & $0-20$ & 1.34 & 0.77 & 0.068 & 11.43 & 4.1 & 4.4 & 7.1 & 2.4 & 0.4 \\
\hline Szymaniszki C & $20-40$ & - & - & - & - & 4.2 & 4.7 & 2.8 & 1.7 & 1.0 \\
\hline Szymaniszki C & $90-110$ & - & - & - & - & 4.7 & 6.0 & 1.9 & 1.0 & 1.8 \\
\hline
\end{tabular}

\begin{tabular}{|c|c|c|c|c|c|c|c|c|c|c|}
\hline \multirow{2}{*}{ Soil pit } & Sam- & \multirow{3}{*}{$\begin{array}{c}\text { pling } \\
\text { depth }\end{array}$} & $\begin{array}{c}\text { Hydrolytic } \\
\text { acidity } \\
\text { mmol/100g }\end{array}$ & \multicolumn{4}{|c|}{ Exchangeable cations } & \multicolumn{4}{c|}{$\begin{array}{c}\text { Sorption capacity } \\
\text { me/100g }\end{array}$} \\
\cline { 5 - 13 } & & & $\mathrm{Ca}^{2+}$ & $\mathrm{Mg}^{2+}$ & $\mathrm{Na}^{+}$ & $\mathrm{K}^{+}$ & $\mathrm{S}$ & $\mathrm{T}$ & $(\mathrm{T}-\mathrm{S})$ & $\mathrm{V}, \%$ \\
\hline Szymaniszki C & $0-20$ & 5.42 & 0.20 & 0.033 & 0.017 & 0.072 & 0.322 & 5.742 & 5.42 & 5.61 \\
\hline Szymaniszki C & $20-40$ & 3.21 & 0.65 & 0.077 & 0.017 & 0.051 & 0.795 & 4.005 & 3.21 & 19.85 \\
\hline Szymaniszki C & $90-110$ & 1.49 & 1.00 & 0.155 & 0.026 & 0.020 & 1.201 & 2.691 & 1.49 & 44.63 \\
\hline
\end{tabular}

Source: own elaboration.

\section{Characteristics of the flora and fungi - Szymaniszki C}

Approximately $100 \%$ of the study plot is covered with plants. Fairly rich in species of plants (10). Five species dominate: Senecio viscosus (30\%), Crepis biennis (20\%), Padus serotina (20\%), Solidago canadensis $(10 \%)$ and Cirsium arvense $(10 \%)$. The share of other species is low (Tab. 6.1). The area also features specimens of trees and shrubs: Pinus sylvestris, Betula pendula and Padus serotina.
Fungi of the plot are not abundant. They are represented by 6 species of macromycetes. The most frequently found species include: Marasmius oreades and Conocybe tenera. The undergrowth also features: Macrolepiota procera and Panaeolus foenisecii. The mycorrhizal fungi were represented by: Inocybe asterospora and Amanita muscaria (Tab. 7.1).

\section{Analysis of granulometric composition and chemical properties of soils in the Szymaniszki study plot group}

On the basis of the results of grain size distribution analyses (Tab. 5.34-5.36), the soils of abandoned lands in the Szymaniszki study plot group were classified as two granulometric groups of sands and loams (Polskie Towarzystwo Gleboznawcze 2009). At plot Szymaniszki A, the subsequent soil horizons, starting from the surface, were classified as subgroups of loamy sand, sand and loose sand. As regards agrotechnical heaviness, the soil was classified as very light. At plot Szymaniszki B, in the two shallower soil horizons the silty fraction constituted $36.5 \%$ and $42.7 \%$, the deepest horizon was dominated with sand $-94.3 \%$, which provided the base to include them in subgroups of sandy loam and loose sand, respectively. The soil was classified as the 
agronomical category of light soils. At plot Szymaniszki $C$, the soil texture was similar to that of plot $B$, but the content of silts decreased faster with the depth and the subsequent horizons were classified into subgroups of sandy loam, slightly loamy sand and loose sand, respectively. The soil's agrotechnical heaviness was classified as light soils.

At plot Szymaniszki B, in the $20-40 \mathrm{~cm}$ horizon, the index of specific surface area, dependent on the content of the silty and clayey fraction, reached its highest value of $0.402 \mathrm{~m}^{2} / \mathrm{g}$.

The content of humus was medium and ranged from $1.24 \%$ at plot A to $1.86 \%$ at plot C. The lowest value was recorded in the soil formed on loamy sand, and the highest - on sandy loam. The $\mathrm{C} / \mathrm{N}$ ratio was narrow: 12.6:1-10.4:1.

The $\mathrm{pH}$ value in $1 \mathrm{M} \mathrm{KCl}$ in all surface horizons of the soils indicated very acidic reaction and acidic reaction in deeper horizons.

The surface horizon of soils of the Szymaniszki study plot group was characterised by low content of available phosphorus, whereas deeper horizons had very low content of this element. The soil in the surface horizon at plot Szymaniszki $\mathrm{C}$ was characterised by low content of potassium, and the remaining analysed horizons - by very low content. The content of magnesium at all plots and soil horizons increased with the depth of the profile from very low to medium at plot B and low at plot $\mathrm{C}$.

The level of hydrolytic acidity clearly decreased with depth, whereas total alkaline cations, generally low, revealed fluctuations in various directions within the range from 0.322 to $2.096 \mathrm{mmol} / 100 \mathrm{~g}$. Among alkaline cations, the most important at all plots was calcium. Total sorptive capacity of the soils was very low: it reached the maximum of $6.486 \mathrm{mmol} / 100 \mathrm{~g}$ at plot Szymaniszki B, in the humus horizon. The degree of soil saturation with alkaline cations was different at different sites and horizons, but generally increased with depth and $\mathrm{pH}$ value to reach the highest values at plots $\mathrm{B}-75.9 \%$ and $\mathrm{A}-62.9 \%$. At plot $\mathrm{C}$, the saturation of alkaline cations was clearly the lowest and ranged from $5.61 \%$ to $44.6 \%$.

\section{ŁAGIEWNIKI STUDY PLOT GROUP}

The Łagiewniki study plot group is located about $450 \mathrm{~m}$ to the north of the border of the Łódź Hills Landscape Park, in Zgierz District, Rural Commune of Zgierz, on its border with Łódź. According to the physicogeographical regionalisation (Kondracki 2002), it is located in the north-western part of the mesoregion of the Łódź Hills, within the macroregion of Southern Mazovian Hills.

The analysed area lies in the suburban zone of Łódź and Zgierz. It contains alternating strips of arable lands, abandoned farmlands and narrow strips of shrubs and forests, which are perpendicular to the dirt road running across the area. The southern border of the area is the voivodeship road 71 . On the other sides, it is limited by developing suburban villages: Skotniki, Smardzew and Łagiewniki Nowe.

The analysed area lies at the elevation of approximately 200-222 m a.s.l. in the extreme north-western part of the highest marginal level of the Łódź Upland (Klatkowa 1972). According to the Detailed Geological Map of Poland, its geological substratum is constituted by upper fluvioglacial sands and sands with gravels of the Warta Glaciation (Klatkowa et al. 1991). On the basis of soil and agricultural maps (http://geoportal. lodzkie.pl/imap/), the soils are identified as le- eched brown. In study plots Łagiewniki A and B, the soils were formed from loose sands and were classified as very poor rye complex (7) of agricultural suitability. Study plot Łagiewniki $C$ is located on the border of loamy silty sands upon slightly loamy sands and loam, and loamy silty sands upon loose sand. Respectively, leeched brown soils at this plot, classified as good rye complex (5), turn into poor rye complex (6). Soils of plot $A$ and $B$ are qualified as valuation class VI, and soils at plot Łagiewniki $\mathrm{C}$ - as valuation class $\mathrm{V}$ of arable lands. The soil and agricultural map shows the presence of a stratum with features of periglacial cover sands and silts (Krysiak 2006b).

All the study plots: Łagiewniki A, B and C are within a type 6 geocomplex - glacial and fluvioglacial boulders, cobbles, gravels, sands and muds of plateaus, alluvial fans and erosional-accumulational terraces. It belongs to the group of lithogenic geocomplexes, which normally do not undergo excessive moisturising, associated with permeable Quaternary deposits.

The physiognomy of the study plot group and soil profiles are illustrated with figures 5.67-5.72. Results of laboratory soil analyses are presented in tables 5.37-5.39. 
STUDY PLOT ŁAGIEWNIKI A (51 ${ }^{\circ} 51^{\prime} 360 \mathrm{~N}, 1^{\circ} 28^{\prime} 343 \mathrm{E}$, elevation $222 \mathrm{~m}$ a.s.l.)
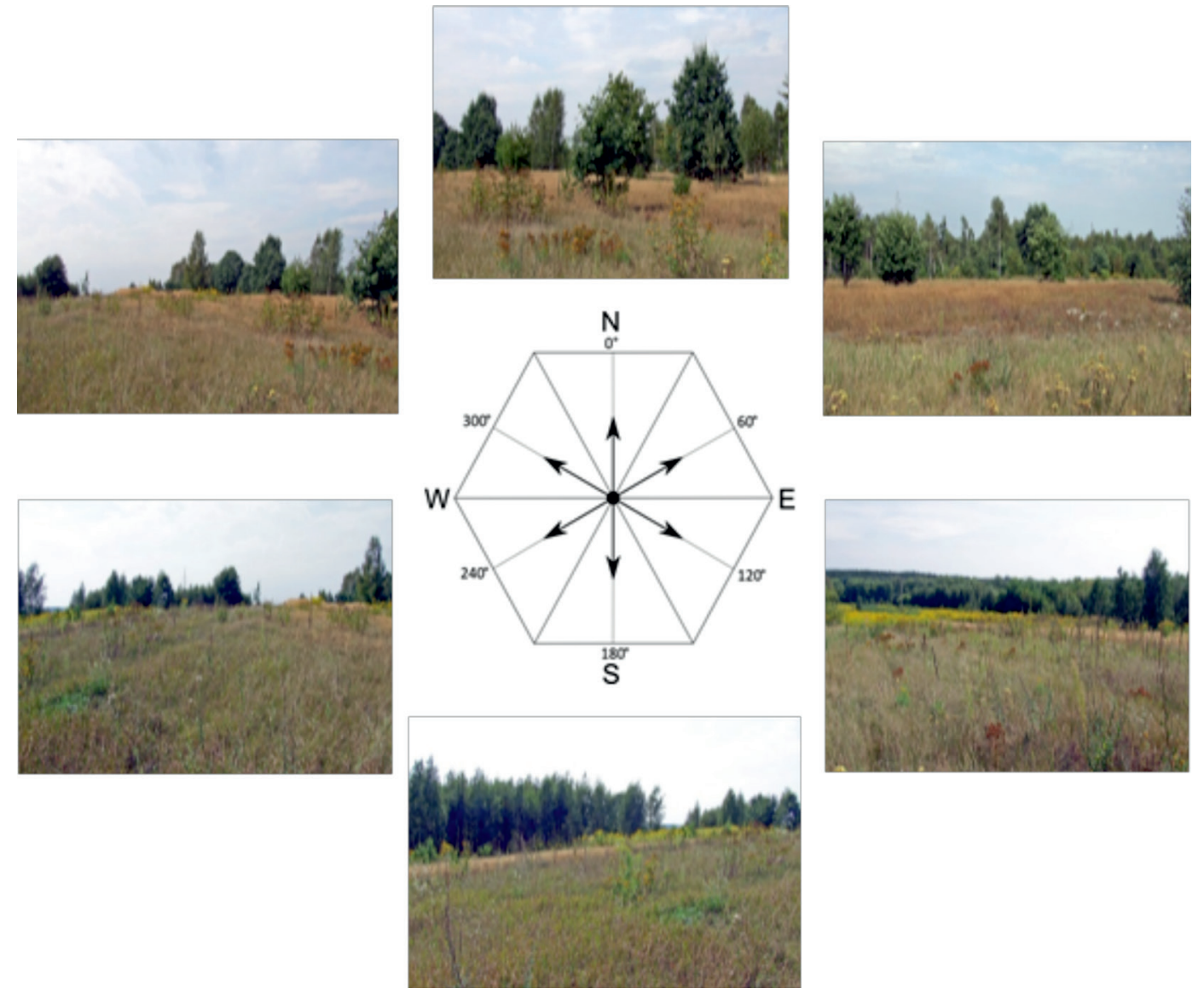

Fig. 5.67. Study plot Łagiewniki A (photo A. Majchrowska, 2013)

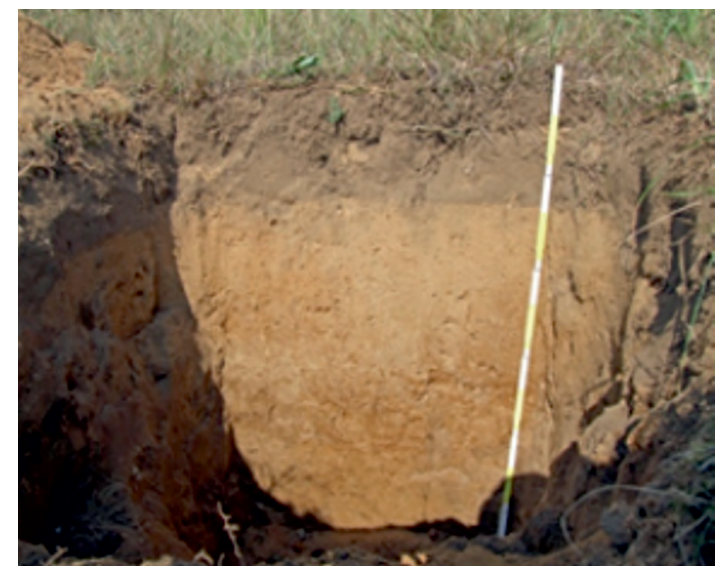

Depth Profile description

0-29 cm - humus, unsorted sand, silty with cobbles, dark brown and grey

29-60 cm - coarse and medium sand with gravel and cobbles, silty, grey and dark brown

60-100 cm - coarse sand with gravel and cobbles, grey, dark brown and yellow

100-150 cm - medium sand with gravel, yellowish

150-160 cm - medium and unsorted sand, yellowish

Fig. 5.68. Soil pit in study plot Łagiewniki A (photo A. Majchrowska, 2013) 
Table 5.37. Study plot Łagiewniki A. Granulometric and chemical properties of soil

\begin{tabular}{|c|c|c|c|c|c|c|c|c|c|c|c|c|}
\hline \multirow[b]{2}{*}{ Soil pit } & \multirow{2}{*}{$\begin{array}{c}\text { Sampling } \\
\text { depth } \\
\text { cm }\end{array}$} & \multicolumn{9}{|c|}{$\begin{array}{c}\text { Grain size distribution } \\
\% \\
\end{array}$} & \multirow{2}{*}{$\begin{array}{l}\text { Gra- } \\
\text { nulo- } \\
\text { metric } \\
\text { sub- } \\
\text { group }\end{array}$} & \multirow{2}{*}{$\begin{array}{l}\text { Spe- } \\
\text { cific } \\
\text { surfa- } \\
\text { ce area } \\
\mathrm{m}^{2} \cdot \mathrm{g}^{-1}\end{array}$} \\
\hline & & $\begin{array}{l}2.0- \\
1.0 \\
\mathrm{~mm}\end{array}$ & $\begin{array}{l}1.0- \\
0.5 \\
\mathrm{~mm}\end{array}$ & $\begin{array}{l}0.5- \\
0.25 \\
\mathrm{~mm}\end{array}$ & $\begin{array}{c}0.25- \\
0.1 \\
\mathrm{~mm}\end{array}$ & $\begin{array}{l}0.1- \\
0.05 \\
\mathrm{~mm}\end{array}$ & $\begin{array}{l}0.05- \\
0.02 \\
\mathrm{~mm}\end{array}$ & $\begin{array}{c}0.02- \\
0.005 \\
\mathrm{~mm}\end{array}$ & $\begin{array}{c}0.005- \\
0.002 \\
\mathrm{~mm}\end{array}$ & $\begin{array}{c}<0.002 \\
\mathrm{~mm}\end{array}$ & & \\
\hline Łagiewniki A & $0-20$ & 4.3 & 30.5 & 35.0 & 16.9 & 4.3 & 4.5 & 3.2 & 1.0 & 0.4 & $\mathrm{p}$ & 0.0835 \\
\hline Łagiewniki A & $20-40$ & 6.5 & 44.6 & 37.5 & 7.9 & 1.1 & 0.7 & 1.2 & 0.5 & 0.0 & $\mathrm{p}$ & 0.0313 \\
\hline Łagiewniki A & $90-110$ & 9.7 & 49.2 & 33.5 & 2.7 & 1.3 & 1.0 & 1.8 & 0.7 & 0.1 & $\mathrm{p}$ & 0.0420 \\
\hline
\end{tabular}

\begin{tabular}{|c|c|c|c|c|c|c|c|c|c|c|}
\hline \multirow{2}{*}{ Soil pit } & \multirow{2}{*}{$\begin{array}{c}\text { Sampling } \\
\text { depth } \\
\mathrm{cm}\end{array}$} & \multirow{2}{*}{$\begin{array}{c}\text { Humus } \\
\%\end{array}$} & \multirow{2}{*}{$\begin{array}{c}\text { C total } \\
\%\end{array}$} & \multirow{2}{*}{$\begin{array}{c}\mathrm{N} \text { total } \\
\%\end{array}$} & \multirow{2}{*}{$\mathrm{C} / \mathrm{N}$} & \multicolumn{2}{|c|}{$\begin{array}{l}\mathrm{pH} \\
\text { in }\end{array}$} & \multicolumn{3}{|c|}{$\begin{array}{l}\text { Available nutrients } \\
\mathrm{mg} / 100 \mathrm{~g}\end{array}$} \\
\hline & & & & & & $\mathrm{KCl}$ & $\mathrm{H}_{2} \mathrm{O}$ & $\mathrm{P}_{2} \mathrm{O}_{5}$ & $\mathrm{~K}_{2} \mathrm{O}$ & $\mathrm{Mg}$ \\
\hline Łagiewniki A & $0-20$ & 0.88 & 0.5104 & 0.043 & 11.87 & 5.4 & 6.4 & 9.2 & 4.2 & 4.8 \\
\hline Łagiewniki A & $20-40$ & - & - & - & - & 5.3 & 6.5 & 4.6 & 2.8 & 2.9 \\
\hline Łagiewniki A & $90-110$ & - & - & - & - & 4.8 & 5.9 & 3.2 & 1.6 & 2.3 \\
\hline
\end{tabular}

\begin{tabular}{|c|c|c|c|c|c|c|c|c|c|c|}
\hline \multirow[t]{2}{*}{ Soil pit } & \multirow{2}{*}{$\begin{array}{c}\text { Sampling } \\
\text { depth } \\
\text { cm }\end{array}$} & \multirow{2}{*}{$\begin{array}{l}\text { Hydrolytic } \\
\text { acidity } \\
\text { mmol/100g }\end{array}$} & \multicolumn{4}{|c|}{$\begin{array}{c}\text { Exchangeable cations } \\
\text { me } / 100 \mathrm{~g}\end{array}$} & \multicolumn{4}{|c|}{$\begin{array}{c}\text { Sorption capacity } \\
\text { me } / 100 \mathrm{~g}\end{array}$} \\
\hline & & & $\mathrm{Ca}^{2+}$ & $\mathrm{Mg}^{2+}$ & $\mathrm{Na}^{+}$ & $\mathrm{K}^{+}$ & $S$ & $\mathrm{~T}$ & $(\mathrm{~T}-\mathrm{S})$ & $\mathrm{V}, \%$ \\
\hline Łagiewniki A & $0-20$ & 1.76 & 1.70 & 0.410 & 0.035 & 0.100 & 2.245 & 4.005 & 1.76 & 43.95 \\
\hline Łagiewniki A & $20-40$ & 0.94 & 0.65 & 0.255 & 0.009 & 0.062 & 0.976 & 1.916 & 0.94 & 50.94 \\
\hline Łagiewniki A & $90-110$ & 0.71 & 1.15 & 0.193 & 0.017 & 0.028 & 1.388 & 2.098 & 0.71 & 66.16 \\
\hline
\end{tabular}

Source: own elaboration.

\section{Characteristics of the flora and fungi - Łagiewniki A}

Approximately $100 \%$ of the study plot is covered with plants. Medium abundance of plant species (11). Two species dominate: Agrostis capillaris (70\%) and Rumex acetosella (20\%). The share of other species is low and does not exceed $0.5 \%$ of area coverage. Other identified species of plants include: Hieracium pilosella, Poa pratensis, Elymus repens, Spergula arvensis (Tab. 6.1).
The plot features specimens of trees and shrubs: Betula pendula, Quercus robur and Padus serotina.

Fungi of the plot are sparse. They are represented by 4 species of macromycetes. The most frequently found species is Marasmius oreades. The undergrowth also features: Conocybe rickeaniana, Macrolepiota procera and Panaeolus foenisecii (Tab. 7.1). 


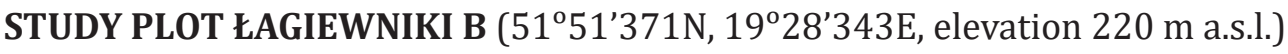
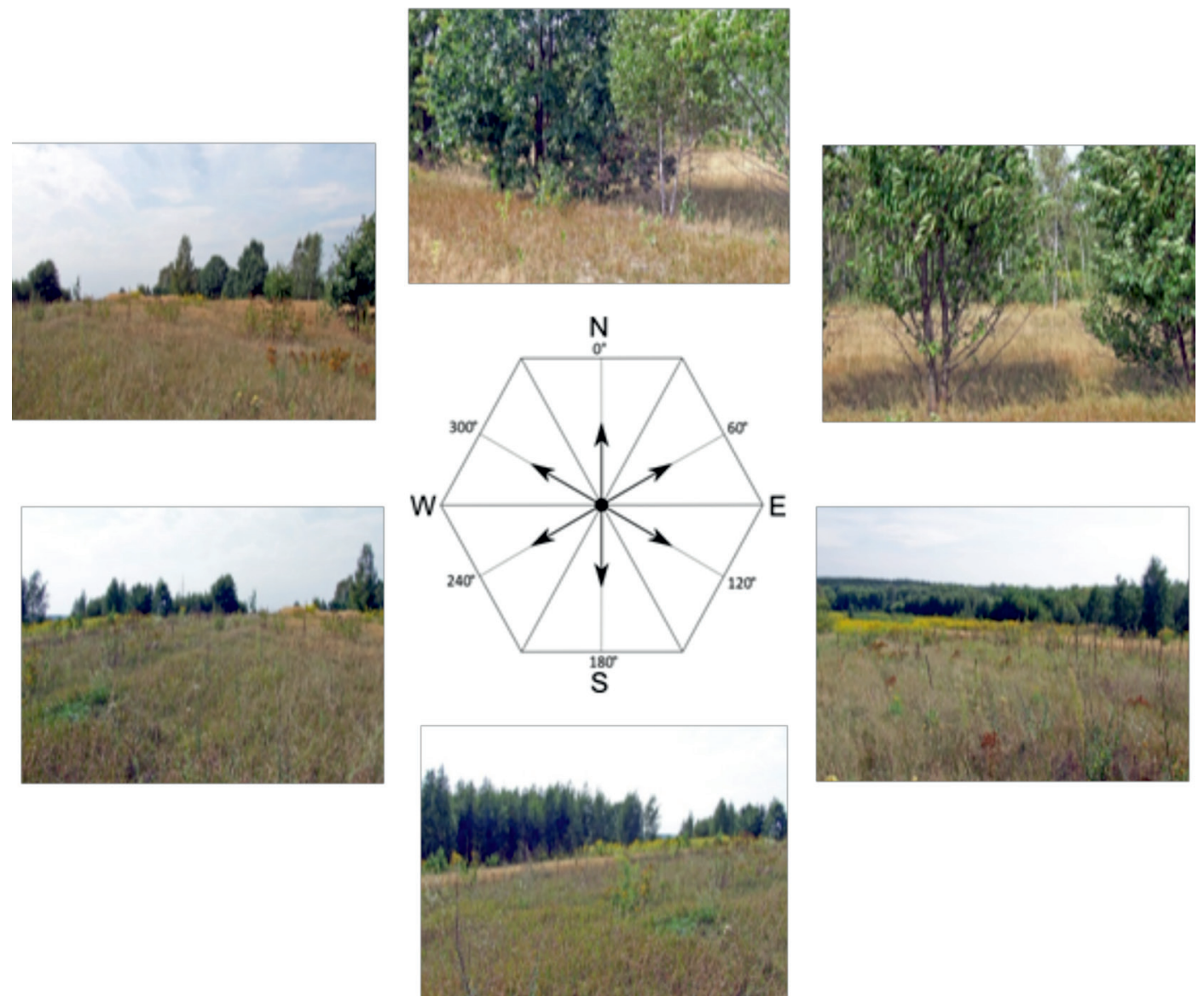

Fig. 5.69. Study plot Łagiewniki B (photo A. Majchrowska, 2013)

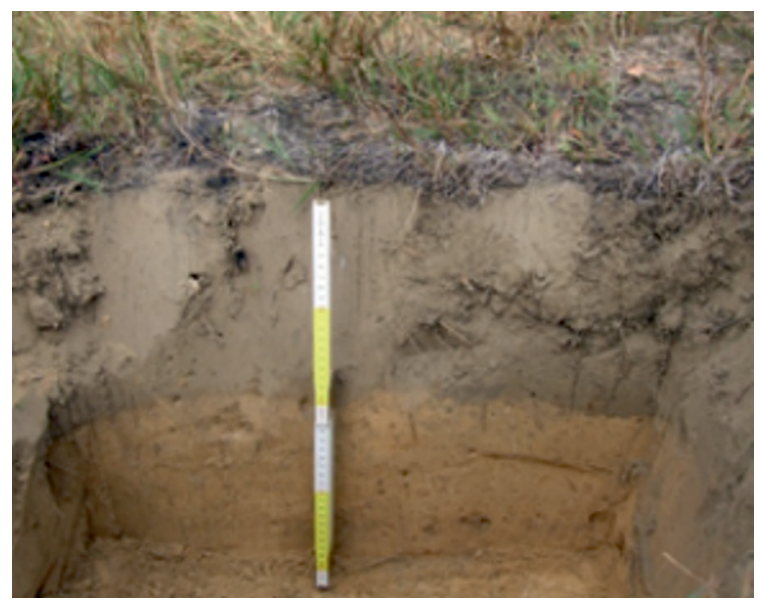

Depth Profile description

$0-23 \mathrm{~cm}$ - humus, silty sand, brown and grey

23-40 cm - silty sand, beige, with single cobbles of up to $3 \mathrm{~cm}$ in diameter at the bottom

$40-45 \mathrm{~cm}$ - cobbles of over $3 \mathrm{~cm}$ in diameter

45-60 cm - silty sand, beige and golden

$60-120 \mathrm{~cm}$ - fine and silty sand, light beige and golden, with single cobbles

120-150 cm - medium and fine sand, silty, light beige and golden

Fig. 5.70. Soil pit in study plot Łagiewniki B (photo A. Majchrowska, 2013) 
Table 5.38. Study plot Łagiewniki B. Granulometric and chemical properties of soil

\begin{tabular}{|c|c|c|c|c|c|c|c|c|c|c|c|c|}
\hline \multirow[b]{2}{*}{ Soil pit } & \multirow{2}{*}{$\begin{array}{c}\text { Sampling } \\
\text { depth } \\
\text { cm }\end{array}$} & \multicolumn{9}{|c|}{$\begin{array}{c}\text { Grain size distribution } \\
\% \\
\end{array}$} & \multirow{2}{*}{$\begin{array}{l}\text { Gra- } \\
\text { nulo- } \\
\text { metric } \\
\text { sub- } \\
\text { group }\end{array}$} & \multirow{2}{*}{$\begin{array}{c}\text { Spe- } \\
\text { cific } \\
\text { surfa- } \\
\text { ce area } \\
\mathrm{m}^{2} \cdot \mathrm{g}^{-1}\end{array}$} \\
\hline & & $\begin{array}{l}2.0- \\
1.0 \\
\mathrm{~mm}\end{array}$ & $\begin{array}{l}1.0- \\
0.5 \\
\mathrm{~mm}\end{array}$ & $\begin{array}{l}0.5- \\
0.25 \\
\mathrm{~mm}\end{array}$ & $\begin{array}{c}0.25- \\
0.1 \\
\mathrm{~mm}\end{array}$ & $\begin{array}{l}0.1- \\
0.05 \\
\mathrm{~mm}\end{array}$ & $\begin{array}{l}0.05- \\
0.02 \\
\mathrm{~mm}\end{array}$ & $\begin{array}{c}0.02- \\
0.005 \\
\mathrm{~mm}\end{array}$ & $\begin{array}{c}0.005- \\
0.002 \\
\mathrm{~mm}\end{array}$ & $\begin{array}{c}<0.002 \\
\mathrm{~mm}\end{array}$ & & \\
\hline Łagiewniki B & $0-20$ & 0.1 & 13.4 & 27.9 & 17.7 & 11.9 & 16.9 & 8.2 & 2.1 & 1.8 & gp & 0.258 \\
\hline Łagiewniki B & $20-40$ & 0.1 & 12.2 & 22.1 & 12.6 & 14.0 & 20.3 & 11.6 & 3.7 & 3.3 & gp & 0.412 \\
\hline Łagiewniki B & $90-110$ & 0.0 & 11.3 & 53.3 & 28.5 & 0.4 & 2.8 & 2.1 & 1.0 & 0.4 & $\mathrm{p}$ & 0.076 \\
\hline
\end{tabular}

\begin{tabular}{|c|c|c|c|c|c|c|c|c|c|c|}
\hline \multirow[t]{2}{*}{ Soil pit } & \multirow{2}{*}{$\begin{array}{c}\text { Sampling } \\
\text { depth } \\
\mathrm{cm}\end{array}$} & \multirow{2}{*}{$\begin{array}{c}\text { Humus } \\
\%\end{array}$} & \multirow{2}{*}{$\begin{array}{c}\text { C total } \\
\%\end{array}$} & \multirow{2}{*}{$\begin{array}{l}\mathrm{N} \text { total } \\
\%\end{array}$} & \multirow[t]{2}{*}{$\mathrm{C} / \mathrm{N}$} & \multicolumn{2}{|c|}{$\begin{array}{l}\mathrm{pH} \\
\text { in }\end{array}$} & \multicolumn{3}{|c|}{$\begin{array}{c}\text { Available nutrients } \\
\mathrm{mg} / 100 \mathrm{~g}\end{array}$} \\
\hline & & & & & & $\mathrm{KCl}$ & $\mathrm{H}_{2} \mathrm{O}$ & $\mathrm{P}_{2} \mathrm{O}_{5}$ & $\mathrm{~K}_{2} \mathrm{O}$ & $\mathrm{Mg}$ \\
\hline Łagiewniki B & $0-20$ & 1.35 & 0.783 & 0.072 & 10.88 & 4.1 & 4.4 & 3.2 & 0.9 & 0.4 \\
\hline Łagiewniki B & $20-40$ & - & - & - & - & 4.3 & 4.5 & 1.8 & 0.6 & 0.3 \\
\hline Łagiewniki B & $90-110$ & - & - & - & - & 4.4 & 5.1 & 1.7 & 0.5 & 0.6 \\
\hline
\end{tabular}

\begin{tabular}{|c|c|c|c|c|c|c|c|c|c|c|}
\hline \multirow[t]{2}{*}{ Soil pit } & \multirow{2}{*}{$\begin{array}{c}\text { Sampling } \\
\text { depth } \\
\mathrm{cm}\end{array}$} & \multirow{2}{*}{$\begin{array}{l}\text { Hydrolytic } \\
\text { acidity } \\
\text { mmol/100g }\end{array}$} & \multicolumn{4}{|c|}{$\begin{array}{c}\text { Exchangeable cations } \\
\text { me } / 100 \mathrm{~g}\end{array}$} & \multicolumn{4}{|c|}{$\begin{array}{l}\text { Sorption capacity } \\
\text { me/100g }\end{array}$} \\
\hline & & & $\mathrm{Ca}^{2+}$ & $\mathrm{Mg}^{2+}$ & $\mathrm{Na}^{+}$ & $\mathrm{K}^{+}$ & $\mathrm{S}$ & $\mathrm{T}$ & (T-S) & $\mathrm{V}, \%$ \\
\hline Łagiewniki B & $0-20$ & 6.24 & 0.15 & 0.027 & 0.013 & 0.036 & 0.226 & 6.466 & 6.24 & 3.495 \\
\hline Łagiewniki B & $20-40$ & 4.54 & 0.15 & 0.020 & 0.009 & 0.026 & 0.205 & 4.745 & 4.54 & 4.320 \\
\hline Łagiewniki B & $90-110$ & 1.57 & 0.30 & 0.053 & 0.070 & 0.015 & 0.438 & 2.008 & 1.57 & 21.810 \\
\hline
\end{tabular}

Source: own elaboration.

\section{Characteristics of the flora and fungi - Kagiewniki B}

Approximately $70 \%$ of the study plot is covered with plants. Poor in species of plants (9). 6 species occur with similar area coverage (about 10\%-15\%): Betula pendula, Elymus repens, Jasione montana, Poa trivialis, Padus serotina and Senecio jacobea. Specimens of other species occur in small quantities (Tab. 6.1).
Fungi of the study plot are not abundant. They are represented by 4 species of macromycetes. The most frequently found species include: Marasmius oreades and Panaeolus foenisecii. Mycorrhizal fungi are represented by: Inocybe asterospora and Leccinum scabrum (Tab. 7.1). 
STUDY PLOT ŁAGIEWNIKI C (51 $51^{\circ} 496 \mathrm{~N}, 19^{\circ} 28^{\prime} 400 \mathrm{E}$, elevation $214 \mathrm{~m}$ a.s.l.)
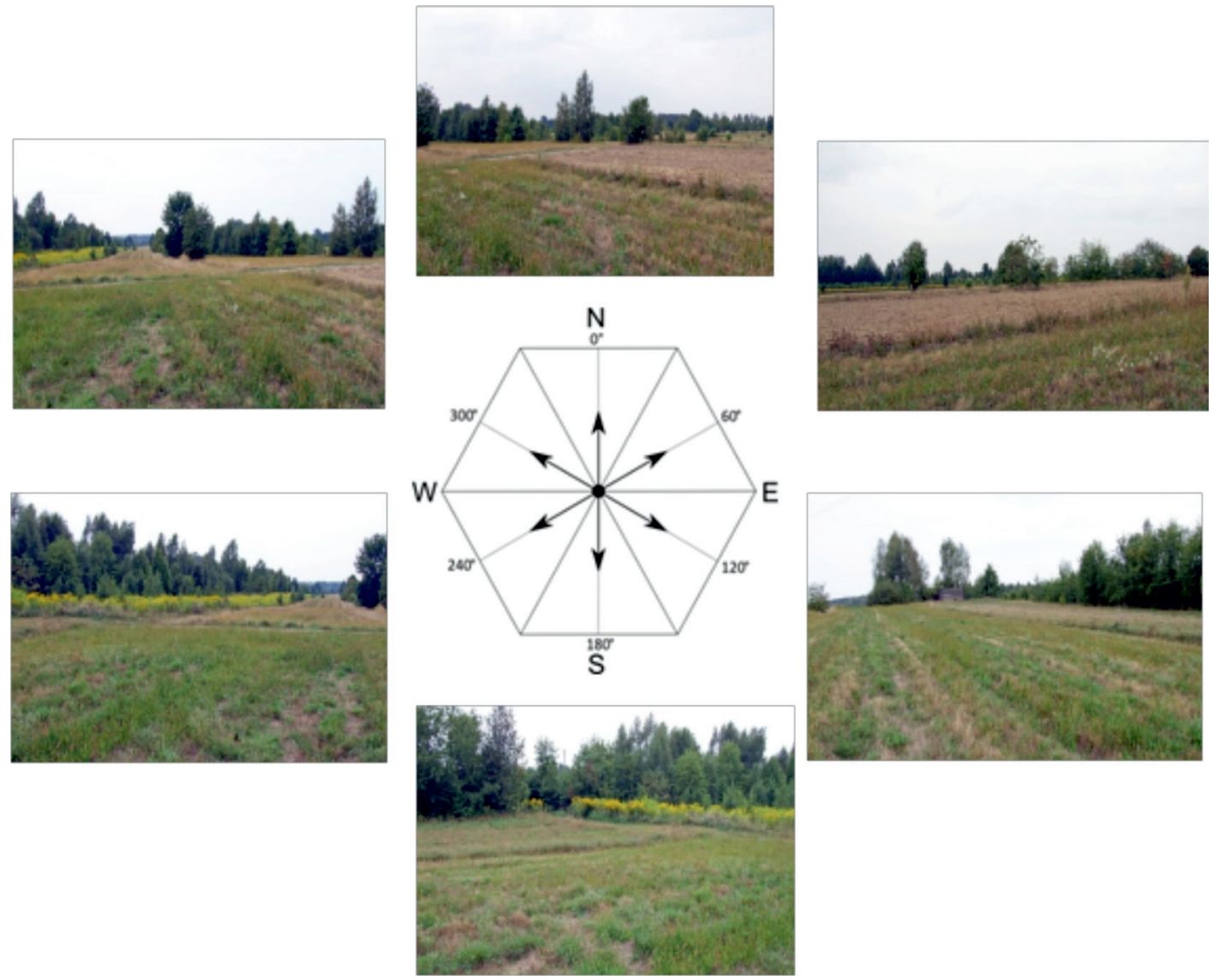

Fig. 5.71. Study plot Łagiewniki C (photo A. Majchrowska, 2013)

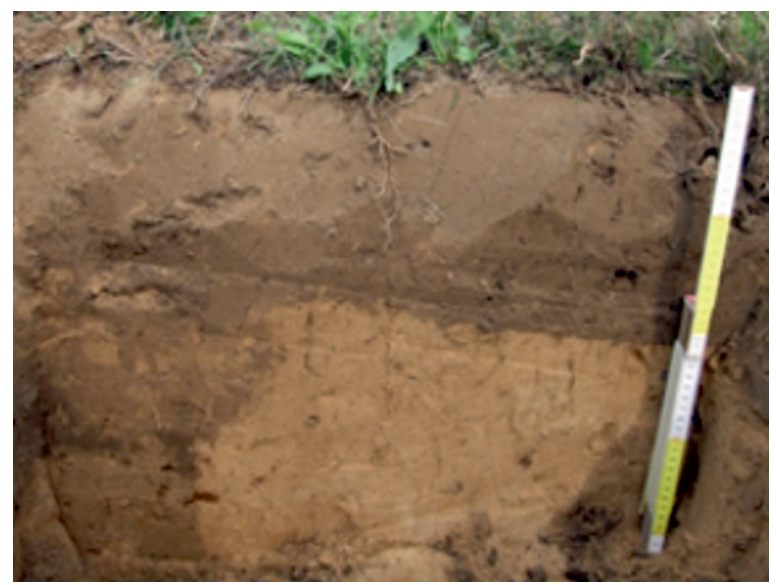

Depth Profile description

0-21 cm - humus, silty sand, dark brown

21-40 cm - unsorted silty sand, yellow

$40-45 \mathrm{~cm}$ - fine sand, silty, golden

45-110 cm - unsorted sand, slightly loamy, rusty and golden

$110-150 \mathrm{~cm}$ - fine and medium sand, silty, golden

Fig. 5.72. Soil pit in study plot Łagiewniki C (photo A. Majchrowska, 2013) 
Table 5.39. Study plot Łagiewniki C. Granulometric and chemical properties of soil

\begin{tabular}{|c|c|c|c|c|c|c|c|c|c|c|c|c|}
\hline \multirow[b]{2}{*}{ Soil pit } & \multirow{2}{*}{$\begin{array}{c}\text { Sampling } \\
\text { depth } \\
\text { cm }\end{array}$} & \multicolumn{9}{|c|}{$\begin{array}{c}\text { Grain size distribution } \\
\%\end{array}$} & \multirow{2}{*}{$\begin{array}{l}\text { Gra- } \\
\text { nulo- } \\
\text { metric } \\
\text { sub- } \\
\text { group }\end{array}$} & \multirow{2}{*}{$\begin{array}{c}\text { Spe- } \\
\text { cific } \\
\text { surfa- } \\
\text { ce area } \\
\mathrm{m}^{2} \cdot \mathrm{g}^{-1}\end{array}$} \\
\hline & & $\begin{array}{l}2.0- \\
1.0 \\
\mathrm{~mm}\end{array}$ & $\begin{array}{l}1.0- \\
0.5 \\
\mathrm{~mm}\end{array}$ & $\begin{array}{l}0.5- \\
0.25 \\
\mathrm{~mm}\end{array}$ & $\begin{array}{c}0.25- \\
0.1 \\
\mathrm{~mm}\end{array}$ & $\begin{array}{l}0.1- \\
0.05 \\
\mathrm{~mm}\end{array}$ & $\begin{array}{l}0.05- \\
0.02 \\
\mathrm{~mm}\end{array}$ & $\begin{array}{c}0.02- \\
0.005 \\
\mathrm{~mm}\end{array}$ & \begin{tabular}{c|}
$0.005-$ \\
0.002 \\
$\mathrm{~mm}$
\end{tabular} & $\begin{array}{c}<0.002 \\
\mathrm{~mm}\end{array}$ & & \\
\hline Łagiewniki C & $0-20$ & 4.8 & 27.8 & 23.9 & 7.6 & 9.7 & 13.6 & 8.3 & 2.5 & 2.0 & pg & 0.266 \\
\hline Łagiewniki C & $20-40$ & 0.7 & 26.0 & 39.3 & 15.7 & 5.7 & 5.9 & 4.3 & 1.4 & 0.9 & $\mathrm{p}$ & 0.136 \\
\hline Łagiewniki C & $90-110$ & 3.4 & 22.7 & 28.4 & 26.9 & 7.5 & 3.5 & 4.4 & 2.1 & 1.1 & $\mathrm{p}$ & 0.159 \\
\hline Łagiewniki C & $150-160$ & 0.7 & 14.3 & 26.7 & 33.1 & 12.7 & 5.2 & 4.3 & 1.8 & 1.2 & ps & 0.172 \\
\hline
\end{tabular}

\begin{tabular}{|c|c|c|c|c|c|c|c|c|c|c|}
\hline \multirow{2}{*}{ Soil pit } & \multirow{2}{*}{$\begin{array}{c}\text { Sampling } \\
\text { depth } \\
\text { cm }\end{array}$} & \multirow{2}{*}{$\begin{array}{c}\text { Humus } \\
\%\end{array}$} & \multirow{2}{*}{$\begin{array}{c}\text { C total } \\
\%\end{array}$} & \multirow{2}{*}{$\begin{array}{l}\mathrm{N} \text { total } \\
\%\end{array}$} & \multirow{2}{*}{$\mathrm{C} / \mathrm{N}$} & \multicolumn{2}{|c|}{$\begin{array}{l}\mathrm{pH} \\
\text { in }\end{array}$} & \multicolumn{3}{|c|}{$\begin{array}{c}\text { Available nutrients } \\
\mathrm{mg} / 100 \mathrm{~g}\end{array}$} \\
\hline & & & & & & $\mathrm{KCl}$ & $\mathrm{H}_{2} \mathrm{O}$ & $\mathrm{P}_{2} \mathrm{O}_{5}$ & $\mathrm{~K}_{2} \mathrm{O}$ & $\mathrm{Mg}$ \\
\hline Łagiewniki C & $0-20$ & 1.38 & 0.8004 & 0.068 & 11.77 & 4.2 & 4.6 & 4.5 & 2.5 & 1.0 \\
\hline Łagiewniki C & $20-40$ & - & - & - & - & 4.4 & 5.0 & 3.1 & 1.6 & 0.8 \\
\hline Łagiewniki C & $90-110$ & - & - & - & - & 4.2 & 5.3 & 3.1 & 1.6 & 2.8 \\
\hline Łagiewniki C & $150-160$ & - & - & - & - & 4.2 & 4.9 & 4.1 & 1.3 & 2.5 \\
\hline
\end{tabular}

\begin{tabular}{|c|c|c|c|c|c|c|c|c|c|c|}
\hline \multirow[t]{2}{*}{ Soil pit } & \multirow{2}{*}{$\begin{array}{c}\text { Sampling } \\
\text { depth } \\
\mathrm{cm}\end{array}$} & \multirow{2}{*}{$\begin{array}{l}\text { Hydrolytic } \\
\text { acidity } \\
\text { mmol/100g }\end{array}$} & \multicolumn{4}{|c|}{$\begin{array}{c}\text { Exchangeable cations } \\
\text { me } / 100 \mathrm{~g}\end{array}$} & \multicolumn{4}{|c|}{$\begin{array}{c}\text { Sorption capacity } \\
\text { me } / 100 \mathrm{~g}\end{array}$} \\
\hline & & & $\mathrm{Ca}^{2+}$ & $\mathrm{Mg}^{2+}$ & $\mathrm{Na}^{+}$ & $\mathrm{K}^{+}$ & S & $\mathrm{T}$ & $(\mathrm{T}-\mathrm{S})$ & $\mathrm{V}, \%$ \\
\hline Łagiewniki C & $0-20$ & 4.66 & 0.35 & 0.082 & 0.009 & 0.072 & 0.513 & 5.173 & 4.66 & 9.92 \\
\hline Łagiewniki C & $20-40$ & 2.14 & 0.35 & 0.078 & 0.009 & 0.072 & 0.509 & 2.649 & 2.14 & 19.22 \\
\hline Łagiewniki C & $90-110$ & 1.69 & 1.40 & 0.262 & 0.030 & 0.041 & 1.733 & 3.423 & 1.69 & 50.63 \\
\hline Łagiewniki C & $150-160$ & 1.42 & 1.30 & 0.233 & 0.026 & 0.036 & 2.020 & 3.440 & 1.42 & 58.72 \\
\hline
\end{tabular}

Source: own elaboration.

\section{Characteristics of the flora and fungi - Łagiewniki C}

Approximately $100 \%$ of the study plot is covered with plants. Medium abundance of species of plants (13). Five species dominate: Betula pendula (15\%), Conyza canadensis (10\%), Elymus repens (10\%), Rumex acetosa (10\%). Other species cover about $5 \%$ of the area each. They include: Anagalis arvensis, Geranium pratense, Gnaphalium sylvaticum, Hypericum perforatum, Matricaria chamomilla, Padus serotina (Tab. 6.1).

Fungi of the plot are not abundant. They are represented by 4 species of macromycetes. The most frequently found species was Marasmius oreades. The undergrowth also features Conocybe rickeaniana (Tab. 7.1).

\section{Analysis of granulometric composition and chemical properties of soils in the Lagiewniki study plot group}

On the basis of the results of grain size distribution analyses, soils of the Łagiewniki study plot group were classified as two granulometric groups of sands and loams (Polskie Towarzystwo Gleboznawcze 2009). At plot Łagiewniki A, there is a clear domination of the sandy fraction
(90.9-97.7\%), and within it - of coarse and medium sand, allowed for the soil to be classified as the agronomic category of very light, wellaerated and permeable soils. At plot Łagiewniki $\mathrm{B}$, a discrepancy was noticed between the analysis results and the state presented on the soil and 
agricultural map (http://geoportal.lodzkie.pl/ imap/). Instead of loose sand, in the $0-20 \mathrm{~cm}$ and 20-40 cm horizons, an increase of the content of the silty fraction to $27.3-35.7 \%$ was detected, which provided a basis for classifying the soil with the subgroup of sandy loam. At plot Łagiewniki $C$, the content of silt was $24.3 \%$ in the surface horizon and it remained at the level of $10-11 \%$ in the deeper horizons, which allowed for the soil to be classified with the subgroup of loamy sand, and deeper - loose and slightly loamy sand. Soils of plots $\mathrm{B}$ and $\mathrm{C}$ were classified with the agronomic category of light soils.

Specific surface area, low in study plot A, reached its maximum value of $0.412 \mathrm{~m}^{2} \cdot \mathrm{g}^{-1}$ in the horizon of 20-40 cm at plot Łagiewniki B, where the presence of silty-sandy cover was recorded. The content of humus was low at plot $\mathrm{A}-0.88 \%$ and medium (1.35-1.38\%) at the other plots. The $\mathrm{C} / \mathrm{N}$ ratio was about 11:1-10:1.

The $\mathrm{pH}$ value at plot $\mathrm{A}$ indicated acidic reaction in $1 \mathrm{M} \mathrm{KCl}$, and slightly acidic in $\mathrm{H}_{2} \mathrm{O}$ and decreased with the depth of the profile. At the other plots, the reaction was very acidic.

Soils of the Łagiewniki study plot group were characterised by a very low content of available phosphorus, except for the humus horizon at plot A, where the level was low. All soils were characterised by very low and low content of potassium.
The content of available magnesium at plot $A$ was high and decreased with the depth of the profile to medium. At plot B - it was very low, at plot $\mathrm{C}$ - very low near the surface and increased to medium values in deeper horizons.

The level of hydrolytic acidity clearly decreased with the depth of the profile, whereas total alkaline cations, generally low, revealed a growing tendency with the depth within the range from 0.205 to $2.245 \mathrm{mmol} / 100 \mathrm{~g}$. Among alkaline cations, the most important at all plots was calcium, and then magnesium. Total sorptive capacity of the soils was very low: it reached the maximum value of $6.466 \mathrm{mmol} / 100 \mathrm{~g}$ at plot Łagiewniki $\mathrm{B}$, in the humus horizon. The saturation degree of soils with alkaline cations was different at different sites and horizons, but it generally clearly increased with depth. The saturation degree of soils with alkaline cations was the highest at plot A - 43.95-66.16\%, and the lowest - at plot B - 3.495-21.81\%.

Characteristics of exchangeable sorption and other physical and chemical parameters of the soils indicate diversified, though generally low habitat value of the Łagiewniki study plot group. Despite its less favourable, very light granulometric composition, the soil at plot A was characterised by the relatively highest $\mathrm{pH}$ level, content of alkaline cations and saturation degree of alkaline ions. 